\title{
Exercise without worries : prevention of stress and depressive symptoms in women from disadvantaged communities
}

Citation for published version (APA):

van der Waerden, J. E. B. (2011). Exercise without worries : prevention of stress and depressive symptoms in women from disadvantaged communities. [Doctoral Thesis, Maastricht University]. Universiteit Maastricht. https://doi.org/10.26481/dis.20110908jw

Document status and date:

Published: 01/01/2011

DOI:

10.26481/dis.20110908jw

Document Version:

Publisher's PDF, also known as Version of record

\section{Please check the document version of this publication:}

- A submitted manuscript is the version of the article upon submission and before peer-review. There can be important differences between the submitted version and the official published version of record. People interested in the research are advised to contact the author for the final version of the publication, or visit the DOI to the publisher's website.

- The final author version and the galley proof are versions of the publication after peer review.

- The final published version features the final layout of the paper including the volume, issue and page numbers.

Link to publication

\footnotetext{
General rights rights.

- You may freely distribute the URL identifying the publication in the public portal. please follow below link for the End User Agreement:

www.umlib.nl/taverne-license

Take down policy

If you believe that this document breaches copyright please contact us at:

repository@maastrichtuniversity.nl

providing details and we will investigate your claim.
}

Copyright and moral rights for the publications made accessible in the public portal are retained by the authors and/or other copyright owners and it is a condition of accessing publications that users recognise and abide by the legal requirements associated with these

- Users may download and print one copy of any publication from the public portal for the purpose of private study or research.

- You may not further distribute the material or use it for any profit-making activity or commercial gain

If the publication is distributed under the terms of Article 25fa of the Dutch Copyright Act, indicated by the "Taverne" license above, 


\section{EXERCISE WITHOUT WORRIES \\ Prevention of stress and depressive symptoms in women from disadvantaged communities}


The research presented in this thesis was conducted at the School for Public Health and Primary Care: CAPHRI, Department of Health Promotion, of Maastricht University. CAPHRI participates in the Netherlands School of Primary Care Research CaRe. CAPHRI was classified as 'excellent' by the external evaluation committee of leading international experts that reviewed CAPHRI in December 2010.

The study was financially supported by a grant from the Netherlands Organization for Health Research and Development (ZonMW) Program for Prevention (No. 40160004).

Cover design: Simon van Alphen

Layout : Tiny Wouters

Printed by: F\&N boekservice

ISBN : 9789491098178

(C) Judith van der Waerden, Maastricht 2011

All rights reserved. No part of this publication may be reproduced, or transmitted in any form or by any means, electronic or mechanical, including photocopying, recording, or any information storage and retrieval without prior permission from the author, or, when appropriate the publishers of the papers. 


\section{EXERCISE WITHOUT WORRIES \\ Prevention of stress and depressive symptoms in women from disadvantaged communities}

\section{PROEFSCHRIFT}

ter verkrijging van de graad van doctor aan de Universiteit Maastricht, op gezag van de Rector Magnificus, Prof. mr. G.P.M.F. Mols, volgens besluit van het College van Decanen,

in het openbaar te verdedigen op donderdag 8 september 2011 om 12.00 uur

door

Judith Elisabeth Bertina van der Waerden 


\section{Promotor}

Prof. Dr. C.M.H. Hosman

\section{Copromotores}

Dr. ir. M.W.J. Jansen

Dr. C. Hoefnagels

\section{Beoordelingscommissie}

Prof. Dr. N.K. de Vries (voorzitter)

Dr. M. Droomers (RIVM)

Prof. Dr. K. Horstman

Prof. Dr. F. Smit (Trimbos Instituut, VU Amsterdam)

Prof. Dr. M. de Vries 


\section{Contents}

$\begin{array}{lll}\text { Chapter } 1 \text { General introduction } & 7\end{array}$

PART I PREVENTION OF DEPRESSION IN LOW-SES WOMEN 25

Chapter 2 Which low-SES women from urban neighborhoods are most 27 at risk for depressive symptoms?

Chapter 3 Psychosocial preventive interventions to reduce depressive 45 symptoms in low-SES women at risk: A meta-analysis

Chapter 4 Exploring recruitment, willingness to participate, and retention of low-SES women in stress and depression prevention

PART II EVALUATION OF THE EXERCISE WITHOUT WORRIES COURSE 85

Chapter 5 Do disadvantaged women appreciate a synergetic exercise $\quad 87$ and psycho-educative program? Rationale and process evaluation of the Exercise without Worries course

Chapter 6 A randomized controlled trial of combined exercise and psycho-education for low-SES women: Short- and long-term outcomes in the reduction of stress and depressive symptoms

Chapter 7 Targeting psychosocial mechanisms with a combined exercise and psycho-educative prevention program for low-SES women: Outcomes of a randomized trial

Chapter 8 General discussion

Summary

Samenvatting

Résumé

References

Dankwoord 



\section{Chapter 1}

General introduction 


\section{General introduction}

When aiming to improve the mental health of the general population, a significant goal should be the reduction of mental health inequalities due to socioeconomic (SES) status. Overall, the burden of mental health problems falls disproportionately on the shoulders of those least able to bear it. The concentration of psychological distress among persons who have attained little in the way of schooling, among those whose occupations are at the low end of the status hierarchy, and among those who live in poverty strongly suggests that these situations create circumstances that have been and continue to be detrimental to mental and emotional well-being (Aneshensel, 2009; Pearlin, 1989; Turner \& Lloyd, 1999).

As will be shown in this introductory chapter, low-SES women are a group at risk for the development of depression. Their increased exposure to stressors is considered an important mediating factor in this process. Several barriers impede low-SES women from fully engaging in the currently available offer of preventive activities. However, an innovative strategy to reach disadvantaged women with the provision of mental health services has been developed. These women might be reached through physical exercise, a mechanism which has been found to play an important role in the prevention and treatment of depression. This formed the foundation for the development of 'Exercise without Worries', an intervention targeted at low-SES women that combines psycho-education and physical exercise. The final part of this chapter will give an overview of this dissertation.

\section{Socioeconomic status and health inequalities}

Socioeconomic status or SES can be considered as an aggregate concept indicating one's level of resources or prestige in relation to others and can be defined for an individual, household unit, neighborhood or community (Gallo \& Matthews, 2003). Various measures are applied in social and medical research as indicators for SES, including objective (education, income, social class and wealth) and subjective (financial strain) measures. While many of the SES indicators and measures are interrelated, they are not fully overlapping and may impact health through different pathways (Andersen, Thielen, Nygaard, \& Diderichsen, 2009; Krieger, Williams, \& Moss, 1997). In psychiatric epidemiology and public health research, education is the most common index of social class (Eaton \& Muntaner, 1999). Education's stability in adult life and its efficiency of measurement and good validity are presumably the main 
reasons for its popularity (Muntaner, Eaton, Miech, \& OCampo, 2004). Years of formal education has also been shown to be a good indicator of long-term economic position, since it often precedes and influences employment, work, earnings, and income, thus acting as a key to positions in the stratification system (Fryers, Melzer, \& Jenkins, 2003; Ross \& Mirowsky, 2006).

A consistently replicated finding in the social sciences has been the negative relationship of SES with health, and with higher mortality and morbidity rates in the lowest SES groups (Mackenbach, Kunst, Cavelaars, Groenhof, \& Geurts, 1997). In the past decades, most Western countries, including the Netherlands, have increasingly recognized socioeconomic health inequalities as an important public-health issue (Mackenbach \& Bakker, 2003; Programma commissie SEGV-II, 2001). Still, despite increased awareness and actions to reduce inequalities, there seems to have been no noticeable change in health inequalities over the last 20 years (Dalstra, Kunst, Geurts, Frenken, \& Mackenbach, 2002; Mol et al., 2005).

SES inequalities have also been found in the distribution of mental disorders. One common mental illness is Major Depression, a psychological disorder that profoundly affects personal functioning. Depression can lead to limitations in social, emotional and physical functioning and reduces quality of life (Bijl \& Ravelli, 2000). Furthermore, depression is related to higher medical costs and increased mortality risk (Beekman et al., 2002). About 121 million people are affected worldwide, making it a leading cause of disability. By 2020 depression is projected to become the second leading cause of the global burden of disease (DALYs) in all age categories for both men and women (Üstün, Ayuso-Mateos, Chatterji, Mathers, \& Murray, 2004; WHO, 2001).

From past research it emerges that two groups appear to be particularly affected by this mental health problem, namely persons with a low socioeconomic status and women. For the lowest SES group Odds Ratio's range from 1.81 for depression prevalence to 2.06 for persistence of depression (de Graaf, ten Have, \& van Dorsselaer, 2010; Lorant et al., 2003; Melchior, Chastang, Leclerc, Ribet, \& Rouillon, 2010). While in the general population 12-month prevalence rates are approximately 5-7\% (Demyttenaere et al., 2004), prevalence and incidence rates for women are consistently found to be about twice as high compared to men (Kuehner, 2003; Seedat et al., 2009). Among women in low socioeconomic strata, studies report the risk of depression onset to be at least two to five times as high (ORs between 2.7 and 5.4) compared to women in the general population (Bassuk, Buckner, Perloff, \& Bassuk, 1998; Kahn, Wise, Kennedy, \& Kawachi, 2000). Prevalence of depression has been found to be three times greater among working class, poor women $(18.7 \%)$ as 
compared to middle and upper class women (6.3\%) (Almeida-Filho et al., 2004).

\section{Socioeconomic disadvantage, stress and depression}

In order to reduce depression in disadvantaged women, it is important to understand the underlying mechanisms that may mediate the association between SES and depression. Numerous factors may contribute to depression in women, including developmental, reproductive, hormonal, genetic, and other biological differences (Sterk, Theall, \& Elifson, 2006). While low-SES women may have the same genetic and biological vulnerability to depression as other women, increased stress may account in part for the increased depression prevalence among women living in disadvantaged circumstances (Hauenstein, 1996; Hobfoll, Johnson, Ennis, \& Jackson, 2003). Three major forms of stressors are often named in the literature: life events are acute changes which require major behavioral readjustments within a relatively short period of time (e.g. birth of first child, divorce). Chronic strains are persistent or recurrent demands which require readjustments over prolonged periods of time (e.g. financial stress, marital problems). Finally, the minor irritations and causes of stress which require small behavioral readjustments during the course of a day (e.g. minor arguments with family/colleagues, job strain) are called daily hassles (McDonough \& Walters, 2001; Thoits, 1995). The number and types of stressors as well as the mechanisms for coping with stress may all contribute to an adverse outcome.

The stress-mental health relationship is often understood from a transactional perspective, in which stress is seen as an individual process. However, a more sociological approach is needed to understand the connection between disadvantaged social status and high rates of psychopathology (Katerndahl \& Parchman, 2002). Within this perspective, stress can both be understood as a consequence of one's place in the social system and as a determinant for psychological stress and depression (Aneshensel, 1992). The Stress Process Model, introduced by Pearlin and colleagues (1981) (see Figure 1.1), aims to capture the complexity of this process by focusing on stressors at the individual, family and community level, and has been proven to be flexible by incorporating advancing scientific understanding of the stress-mental health connection (Wheaton, 2010). 


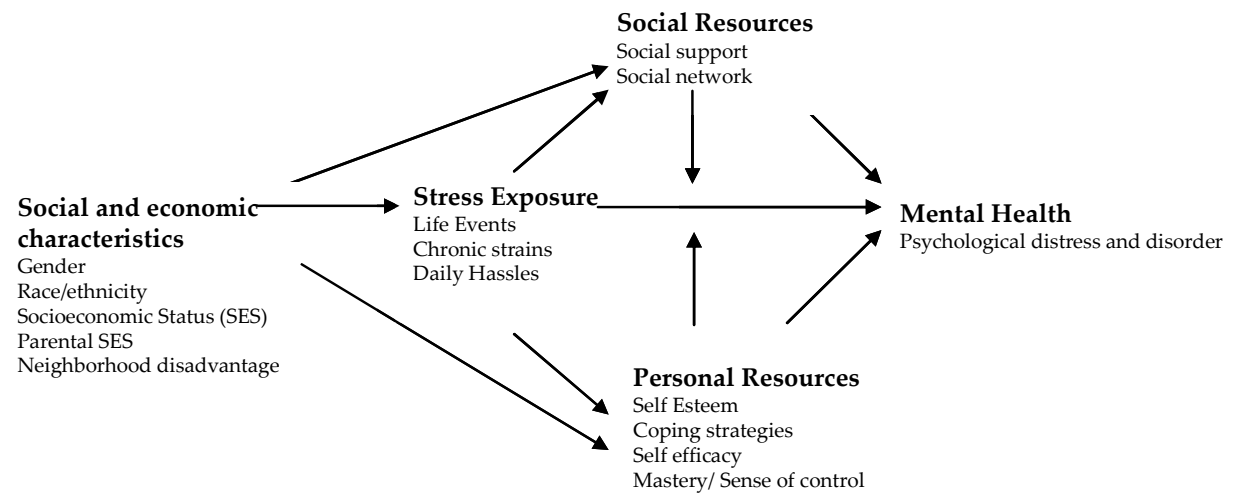

Figure 1.1 Stress Process Model

This model expands on the ways in which social structures come to influence inequalities in mental health and indicates three different, but related pathways through which the stress process can contribute to mental health disparities: (i) differential exposure to stressors; (ii) differential access to psychological and social resources; and (iii) differential effectiveness of psychological and social resources (Pearlin, 1989, 1999a). The primary explanatory variable in this model is social stress, specifically the idea that a low SES decreases mental health because it exposes people to inordinate amounts of stress. In addition to regulating exposure to stressors, disadvantaged social status is seen as limiting access to the mediators and moderators of stress, so that lack of these personal and social resources acts as another route through which disadvantaged social status reduces mental health. Resources that are often considered are self-esteem, coping strategies, self-efficacy, personal mastery and social support (Aneshensel, 2009).

\section{Increased stress exposure}

In most studies, the relationship between stress and risk for depression has been documented for daily hassles, early and recent negative life events and chronic strains (Hammen, 2006; Liu \& Alloy, 2010). Disadvantaged women have been found in several studies to experience more frequent, more threatening and more uncontrollable life events than does the general population, typically in the context of chronic deprivation. They are more often exposed to victimization and violence or sexual exploitation, and gender and racial discrimination (Belle, 1990; WHO, 2000). Although these events are one 
important source of stress, chronic stress and daily hassles are the strongest predictors for both depressive symptoms and depression (Hammen, Kim, Eberhart, \& Brennan, 2009; McConagle \& Kessler, 1990). Chronic stressors are also socially patterned by socio- economic status (Denton, Prus, \& Walters, 2004; Thoits, 1995). For instance, low-SES women are at very high risk of experiencing low income, inadequate housing in dangerous neighborhoods, unemployment, low education, or child-care responsibilities (Belle \& Doucet, 2003; Sterk et al., 2006).

Within the context of SES, life events and chronic strains usually lead to additional stressors (e.g. financial strain, familial dysfunction), a phenomenon referred to as stress proliferation (Katerndahl \& Parchman, 2002; Pearlin, 1999b). Stress proliferation can theoretically involve any combination of life events and chronic stressors (Hammen et al., 2009). While stressors may affect individuals, individuals also act upon their environments, possibly contributing to the stressors they experience. Thus women themselves can contribute actively and substantially to the generation of additional stressors. Hammen (1991, 2006) has suggested that previously depressed individuals' personal characteristics and behaviors generate further stressors in their lives (Shih \& Eberhart, 2007), which in turn increases the likelihood of a chronic course of depression (Liu \& Alloy, 2010).

\section{Differential access to social and personal resources}

In addition to the type of stress or social context in which it occurs, disadvantaged women have fewer and less adequate social and personal resources than more affluent groups (Hobfoll et al., 2003; Miranda \& Green, 1999). The lack of social and personal resources during times of crisis or under stressful life circumstances has been associated with an increased risk of depression among women (Aneshensel, 2009). In particular, deficiencies in resources such as social support and social networks, self- esteem, ruminative coping strategies, self- efficacy and mastery or a sense of personal control over one's life have been linked to the emotional response to stress for lower SES groups (McLeod \& Nonnemaker, 1999; Mrazek \& Haggerty, 1994; Sterk et al., 2006).

One extensively studied resource is social support, with the basic hypothesis being that the impact of stress on mental health is diminished among persons with adequate social support. Social isolation and low levels of social support consistently have been associated with higher levels of stress and depression (Kalil, Born, Kunz, \& Caudill, 2001; Southwick, Vythilingam, \& Charney, 2005). It has been found that social support is generally unequally distributed among social classes, and that fewer and less satisfying social relationships were 
reported in particular by low-SES women (Huurre, Eerola, Rahkonen, \& Aro, 2007). For this group, the presence of support may provide a much-needed resource that promotes a basic sense of control within a stressful environment (Bassuk, Mickelson, Bissell, \& Perloff, 2002).

The relationship between high levels of depressive symptoms and low selfesteem is strong and has consistently been found in the depression literature. Under low levels of stress, it does not matter very much whether people have high or low self-esteem, but when stress levels are high, low self-esteem is usually correlated with the occurrence of depressive episodes (Girgus \& NolenHoeksema, 2006). Disadvantaged women may perceive their current difficulties and past traumas to be a result of worthlessness or their own failure to succeed in a 'makeable' society, and characteristically exhibit low self-esteem (Belle \& Doucet, 2003; Brown, Bifulco, Veiel, \& Andrews, 1990; Thoits, 1995).

Furthermore, in response to stressors women tend to make more use of ruminative coping strategies, defined as focusing on negative mood, negative aspects of self, or stressors (Girgus \& Nolen-Hoeksema, 2006). Ruminative coping strategies have been found to interfere with attention, concentration, and the initiation of instrumental behaviors, increasing depressed mood and contributing to a vicious cycle that maintains and deepens depressive states (Liu \& Alloy, 2010; Peden, Rayens, Hall, \& Grant, 2005). Finally, low-SES women report lower levels of self-efficacy and mastery (Aneshensel, 1992; Gallo \& Matthews, 2003). According to Bandura (1997), self-efficacy suggests that individuals who believe that they can engage in behaviors needed to influence their personal outcomes are more likely to persist at tasks even under conditions of high stress or repeated failure. A closely related and even overlapping concept is mastery, also referred to as internal locus of control or personal control. The concept of mastery suggests a positive expectancy about one's own ability to engage in behaviors needed to cope with a stressful event (Aneshensel, 1992). Persons with lower mastery may behave according to a self-fulfilling prophesy, where they believe they can not exercise control of over their lives and express higher levels of fatalism or demoralization (Pearlin, 1999a; Thoits, 1995). Studies have shown an inverse association between low SES and belief in personal control (Ross \& Mirowsky, 2006; Taylor \& Seeman, 1999). Mastery has been conceptualized as an important resource in dealing with stress and may be understood as an "executive resource" that determines the use of other resources (Green \& Rodgers, 2001). For instance, women high in mastery have been found to appropriately seek and receive higher levels of support (Green \& Rodgers, 2001) and have more self-esteem (Turner \& Lloyd, 1999). 


\section{Erosion of social and personal resources over time}

Social and personal resources are themselves social products, resulting from individuals' socially determined experiences. Because lower SES individuals encounter environmental demands more often, it is likely that these stressors and chronic problems may over time erode the personal resources available to low-SES women (Gallo \& Matthews, 2003). When this occurs, the depleted resources contribute to decreased mental health as well.

Disadvantaged circumstances overstrain not only women's personal resources, but also their social networks and social support (Brown, Ford, Burton, Marshall, \& Dobson, 2005). When women approach their social network with multiple chronic and acute events, it might overwhelm their network's already limited resources and availability (House et al., 1994). Overall, network members will be able to provide less emotional and tangible support (e.g., lower frequency of contact, less emotional support) in times of need (Mickelson \& Kubzansky, 2003). Also, low-SES women may be reluctant to burden their already stressed networks with their own problems, and thus refrain from seeking further social support in stressful times. For those women who report greater access to social support in the form of a confidant, their social networks can serve as conduits of stress too, since members of these social networks are themselves likely to be poor and stressed (Belle \& Doucet, 2003; Elliott, 2001). Although social support, coping and mastery are distinctly different resources, they are interdependent. Because of this interconnection, decreases in one resource are often connected to limitations in the others as well (Pearlin, 1999b).

\section{Differential effectiveness of social and personal resources}

Psychosocial resources may also have a moderating or stress-buffering function and attenuate the magnitude of the relationship between stress and mental health (Aneshensel, 1992). While it has been suggested that low-SES groups make less effective use of their social and personal resources than their higher SES counterparts (Pearlin \& Schooler, 1978), generally these assertions have not been examined empirically. There is no firm scientific basis for assuming that low-SES women are more vulnerable to the mental health effects of stress because they are less successful in utilizing their psychosocial resources - as distinct from having access to fewer resources (Aneshensel, 2009). 


\section{Mental health and low-SES women, a case of double jeopardy}

There are theoretical and empirical indications that the joint effects of female gender and low SES may have particularly deleterious mental health effects (Elliott, 2001; Kuehner, 2003). This simultaneous presence of disadvantageous factors, also referred to as 'double jeopardy', suggests that ill effects of low SES may be more potent for females than males, making women with low socioeconomic status a particularly vulnerable group for the onset and persistence of depression (Mendelson, Kubzansky, Datta, \& Buka, 2008; van de Velde, Bracke, \& Levecque, 2010). Depression may best be conceptualized as a continuum with subclinical depressive symptoms on the one end and a major depressive disorder on the other (Judd, Schettler, \& Akiskal, 2002; Solomon, Haaga, \& Arnow, 2001). Depressive symptoms can in themselves reduce quality of life and increase mental health service use and their presence considerably increases the risk of major depression (Cuijpers \& Smit, 2004; Judd et al., 2002). In studies of disadvantaged women, increased rates (40-50\% of the sample) of depressive symptoms are consistently found (Fleischer, Fernald, \& Hubbard, 2007; Hauenstein, 1996; Thomas, Jones, Scarinci, Mehan, \& Brantley, 2001). Therefore, the prevention of depressive symptoms or reduction of their intensity seems especially relevant for preventing depression incidence in this high risk population (Clarke et al., 2001; Willemse et al., 2004).

\section{Depression prevention in low-SES women}

Since depressive symptoms and stress are highly prevalent in disadvantaged women, their prevention or a reduction of their intensity can contribute to the decrease of a significant public mental health problem (Hauenstein, 1996). The traditional public health classification of prevention defines interventions according to the phases in the disease process they aim to influence. Thus, the term primary prevention refers to activities to reduce the sources and incidence of disease; secondary prevention refers to the amelioration of the disease when it exists, and tertiary prevention to the reduction of complications associated with the presence of a disease.

The Institute of Medicine, following Gordon (1983), introduced new definitions of preventive interventions based on the degree to which the target population is at risk for specific deleterious outcomes, a classification that is habitually applied in the field of mental disorder prevention (Mrazek \& Haggerty, 1994; O'Connell, Boat, \& Warner, 2009). This classification includes universal, selective and indicated interventions. Universal interventions are targeted to the general public or the entire population. Selective interventions 
are aimed at subgroups of the population whose risk of developing a mental disorder is significantly higher than average, as evidenced by biological, psychological or social risk factors (e.g. poverty). Indicated preventive interventions are focused on high-risk persons who have minimal but detectable signs or symptoms forewarning a mental disorder or biological markers indicating a predisposition for a mental disorder but who do not meet diagnostic criteria for disorder yet. In each case, the focus is to lower the incidence of disorders making universal, selective and indicated prevention a form of primary prevention.

The three conceptual areas discussed in the previous section (i.e. exposure to stress, access to resources, and effective use of resources) may help to provide tools for prevention and intervention strategies, since they are to some extent amenable for preventive intervention. Indeed, to achieve these goals, different stress and depression prevention programs have been developed and implemented (Muñoz, Cuijpers, Smit, Barrera, \& Leykin, 2010). However, more specific attention on socioeconomic status in relation to mental health outcomes and the prevention and treatment of depression seems to be called for. As will be shown in the next section, most of the preventive measures for the general public have been shown to have a limited reach and fewer effects among low-SES groups (Busch \& Schrijvers, 2010).

Therefore, in recent years several effective prevention interventions have been developed or adapted to the needs of low-SES women from different cultural backgrounds (e.g. Beeber, Holditch-Davis, Belyea, Funk, \& Canuso, 2004; Muñoz et al., 2007; Peden et al., 2005; Zlotnick, Miller, Pearlstein, Howard, \& Sweeney, 2006). These interventions apply a variety of methods but mostly use psycho-educative and educational techniques (Levy \& O'Hara, 2010). Most of them aim to influence the access, development, and effective use of resources and are examples of selective and indicated prevention. Effect sizes for effectiveness range between 0.07 and 0.42 , depending on the methodology used and inclusion and outcome criteria (Levy \& O'Hara, 2010).

\section{Reaching low-SES women for depression prevention}

Despite advances in depression prevention aimed at low-SES women, a number of problems remain. The main one is that low-SES populations are generally difficult to reach with preventive interventions. Once they have been reached, drop-out from preventive courses is more frequent in disadvantaged groups (Allart- van Dam \& Hosman, 2002), making the retention rate of these women particularly low (Beeber et al., 2007). As low service use patterns among low-SES women can be only partially explained by a relative lack of 
services and resources in lower income communities (Anderson et al., 2006), it is important to understand which barriers to mental health service use might be present for this population.

It is possible to identify some instrumental, psychological and content related barriers to their mental health service use. Concerning the instrumental barriers, the most important are costs, available transportation or child care and time (Azocar, Miranda, \& Dwyer, 1996; Miranda et al., 2006). Since low-income women often live in constrained financial circumstances, costs of services are particularly relevant (Miranda \& Green, 1999). Additional expenses might be incurred for child care fees, discouraging women from attending services (Weinreb, Perloff, Goldberg, Lessard, \& Hosmer, 2006). Many low-SES women do not own cars and are often dependent on relatives or public transport. Weinreb et al (1998) found that lack of transportation prevented over one-fifth of the interviewed women attending mental health services. Finally, clinic hours can be inconvenient for women with low-income service jobs. They are often unable to obtain time off from work to attend treatments (Alvidrez \& Azocar, 1999; Miranda \& Green, 1999). In general, removing instrumental challenges improves service utilization (Anderson et al., 2006).

Beyond these instrumental factors, attitudes and beliefs about mental health issues are psychological barriers that can prohibit service use (Grote, Zuckoff, Swartz, Bledsoe, \& Geibel, 2007). Low income individuals are more likely than middle class persons to express concerns about stigma by community members, employers, health care professionals, and family members if they get help for mental health problems (Alvidrez, 1999). This influences to a large extent their willingness to seek care and continue in treatment (Raingruber, 2002). Additional psychological barriers are linked to a lack of trust in institutions and negative experiences (McLeod, Johnston, \& Griffin, 2000). The former is often related to dissatisfaction with length of waiting time for appointments and discontinuity of care (Jesse, Dolbier, \& Blanchard, 2008; Weinreb et al., 2006). It is suggested that negative experiences in this group stem from possible communication difficulties between service providers and this target group (McLeod et al., 2000). Both lack of trust and negative experiences can lead to a more skeptical and guarded dealings with mental health care providers and service systems (Anderson et al., 2006; Jesse et al., 2008).

Finally, the content of most mental health services may not be perceived as relevant by disadvantaged women (McLeod et al., 2000). In a situation in which they struggle to cope with poor housing, debt or unemployment, mental health care focusing on creating internal changes may make little sense to them when they perceive their distress to be caused by external pressures (Azocar et 
al., 1996; Beeber, Perreira, \& Schwartz, 2008). Previous research has indicated that low-SES women are most interested in short-term, directive, problemsolving psychotherapy and psycho- educational classes about coping with stress or living a healthy life (Alvidrez \& Azocar, 1999; Nadeem, Lange, \& Miranda, 2008). Group counseling, prevention programs and moodmanagement classes are accepted intervention options in this group and can also be seen as ways to minimize stigma when they are presented as psychoeducation rather than therapy (Levy \& O'Hara, 2010; Raingruber, 2002).

To sum up, to include more low-SES women in the prevention of stress and depression it is important to be aware of potential barriers to their mental health service use and where possible tailor approaches and intervention strategies to their needs and values.

\section{Exercise as a means to prevent depression}

It is suggested that non-traditional delivery methods can hold special promise for increasing access to effective mental health interventions (Hollon et al., 2002). Much like other alternative intervention strategies such as acupuncture, herbal approaches, massage and relaxation, exercise as a means to prevent depression has increasingly received interest (Manber, Allen, \& Morris, 2002). According to the WHO public health norms, everybody should be moderately physical active at least 150 minutes throughout the week (WHO, 2010). While regular engagement in physical activity has multiple benefits for the human body, such as reducing the risk for cardiovascular disease, diabetes, cancer and stroke, there is growing evidence as well that physical activity may have both a preventive and a therapeutic impact on mental health problems and stress and has been found to be effective in reducing depressive symptoms (Martinsen, 2008; Ströhle, 2009). Exercise as a preventive treatment for depression has many and various advantages over other preventive methods: exercise may appeal to a broader population, is relatively inexpensive, does not carry a negative stigma, has beneficial effects on other (physical) health aspects and does not have major side effects (Barbour, Edenfield, \& Blumenthal, 2007; Stathopoulou, Powers, Berry, Smits, \& Otto, 2006). In addition, physical activity has increasingly been recommended to individuals with or without disease in order to improve their quality of life (Peluso \& de Andrade, 2005). Thus, exercise is likely to have benefits with few risks and has great potential to impact the treatment and prevention of depression (Dunn et al., 2005).

Many studies have documented the beneficial effects of exercise on positive mental health, including higher self-esteem (Fox, 2000; Penedo \& Dahn, 2005), 
well-being (Hassmén, Koivula, \& Uutela, 2000; Scully, Kremer, Meade, Graham, \& Dudgeon, 1998), quality of life (Atlantis, Chow, Kirby, \& Singh, 2004), stress responsivity (Salmon, 2001; Steptoe, Kimbell, \& Basford, 1998) and anxiety (Bhui \& Fletcher, 2000). Several recent reviews have reported the positive effects of exercise on depressive symptoms across a wide range of populations (Brosse, Sheets, Lett, \& Blumenthal, 2002; Lawlor \& Hopker, 2001; Southwick et al., 2005). Effect sizes ranging between 0.35 and 0.59 have been found for the effects of exercise on the prevalence of depressive symptoms (Conn, 2010). Comparing exercise with other types of treatment, exercise alone was found to be as effective as cognitive therapy, medication and social contacts (Barbour et al., 2007).

Both aerobic and anaerobic exercise forms seem effective to reduce symptoms of depression, while especially the degree of energy-expenditure seems to be important (Anderson et al., 1999; Peluso \& de Andrade, 2005). Most intervention studies suggest that the best psychological benefits are probably derived using a moderate intensity of exercise as opposed to a very low or very high intensity (Goodwin, 2003; Teychnne, Ball, \& Salmon, 2008). Exercise that is more intense than participants' habitual level is less likely to improve mood and is liable to worsen it (Ekkekakis, Hall, \& Petruzzello, 2005). An optimal dose in terms of frequency and duration needed for treatment and prevention efficacy is yet not fully defined (Faulkner, 2009). However, there are some indications that physical activity in the amount recommended by consensus public health norms results in rates of response and remission comparable to those reported in trials of cognitive behavioral therapy and antidepressant medication (Anderson et al., 1999; Brown et al., 2005; Dunn, Trivedi, Kampert, Clark, \& Chambliss, 2005).

\section{Exercise in combination with psychosocial interventions: synergetic effects on depression}

In addition to its application as a monotherapy, exercise is increasingly accepted as an adjunct intervention in the management of depressive symptoms and depression (Merom et al., 2008). Offering multidisciplinary intervention programs is an appealing idea, and often based on the thought that one can never have too much of a good thing. The underlying assumption is that combining different types of effective interventions will increase and prolong their positive effects (Tsang, Chan, \& Cheung, 2008). While several studies on the role of adjunctive exercise in the pharmacologic treatment of depression have shown strong beneficial effects, there has been only limited attention to the combination of exercise with empirically supported 
psychosocial and preventive approaches (e.g., cognitive-behavioral therapy [CBT], interpersonal therapy) (Stathopoulou et al., 2006). Up until now, only a couple of randomized controlled trials have tested the combined effects of cognitive behavioral therapy and exercise on depressive symptoms. Fremont and Craighead (1987) randomly assigned individuals reporting mild to moderate depressive symptoms to one of three treatment groups: (i) a cognitive therapy group (ii) an aerobic exercise group or (iii) a combination of cognitive therapy and exercise. All groups significantly improved after 10 weeks of treatment and maintained their improvement at 4-month follow-up; there were no significant additive effects for exercise relative to CBT alone (Fremont and Craighead, 1987). In a more recent study, the effectiveness of a team-based sport/psychosocial intervention was compared to an individual exercise and a control condition for the mental health of young men. Participants in both the combination group and the individual exercise condition demonstrated a significant decrease in depressive symptom scores compared to the control condition at post-intervention and at 8-week follow-up (McGale, McArdle, \& Gaffney, 2010). However, the sport/psychosocial intervention group did not show any additional effects above the individual exercise group, except for greater perceived social support.

Thus, so far research has not been able find noteworthy synergetic effects for the addition of exercise to psychosocial interventions in depression, but further evaluation of these types of interventions is necessary to draw unequivocal conclusions.

\section{Exercise without Worries intervention}

One commonly used psychological intervention for the indicated prevention of depression is the Coping with Depression course (CWD) (Brown \& Lewinsohn, 1984). The CWD is a flexible, highly structured, cognitive-behavioral intervention, based on the social learning theory, and has been used in group and individual formats, but also as guided self-help and Internet based intervention. Several studies have shown that the CWD course is effective in the prevention of new cases of major depressive disorders (Cuijpers, Munoz, Clarke, \& Lewinsohn, 2009). In the Netherlands, a majority of the Dutch community mental health centers have implemented the Coping With Depression course for different target groups (adults, adolescents, older adults, primary care patients, and ethnic minority groups) (Meijer, Smit, Schoemaker, \& Cuijpers, 2006; Voordouw \& Kramer, 2001). Nevertheless, as indicated earlier, low SES groups profit less from this offer. It appears that participants 
with a lower educational level have more difficulties with the cognitive orientation of the CWD course, due to the high level of required verbal skills and the execution of homework assignments. This results in that low-SES participants drop out of the intervention more often (Allart- van Dam \& Hosman, 2002). Based on the observation that low-SES women do often not engage in depression prevention, innovative ways to offer them preventive activities need to be found. Experience from community programs has shown that exercise is a method suited to reach low-SES women, offering a solution for the low perceived attractiveness of exclusively cognitive-oriented programs among this study population. Based on these premises, the Public Health Service South Limburg and the Community Mental Health Center of Maastricht have developed the Exercise without Worries intervention (from now on referred to as EWW).

The EWW intervention is aimed at women residing in disadvantaged neighborhoods. These women are eligible to participate in the course when they have 10 years of formal education or less (which in the Netherlands equals lower vocational education). To accommodate to the difficulties adult low SESwomen have with the cognitive orientation of depression prevention in general, the contents of the EWW course have been tailored to the specific needs of this target population. The main goal of the intervention is to reduce stress and depressive complaints and to increase coping related competences through improving the balance between burden and capacity. The main focus of the intervention is on empowering the women through their strengths and resilience, instead of focusing on their problems. Psycho-educative programs such as the CWD course usually address risk factors such as negative thought patterns, social skills, self-esteem, and pleasant activities. These topics have been extended with other evidence-based risk factors for stress and depression in the target group of low-SES women. A core element of the EWW course is its group-based format in which the psycho-educative topics link up with bodyfocused exercises. In each session, psycho-education and exercise components are coordinated as far as possible in an effort to reciprocally reinforce the message. This is further elaborated through four themes (I Self-image, II Balance, III Strength, and IV Boundaries) which form the basis of eight twohour sessions (Lanen, Gelissen, Ebben, \& van der Waerden, 2008). Each session is administered by two providers, a licensed psychologist or mental health provider for the psycho-education component and an exercise professional such as a physical therapist or licensed sports instructor. 


\section{Aims and Outline of this dissertation}

The central aim of this dissertation is to explore the possibilities for the prevention of depressive symptoms in disadvantaged women, a population at high-risk for mental health problems. To this aim the first part of this thesis addresses the broader context of prevention of depression in low-SES women. This involves questions as to which women are most likely to benefit from participation in preventive activities, what steps should be undertaken to reach this target group, and what has already been done to reduce depression in this group.

Preventive interventions are often developed from the point of view that prevention of disorders may be achieved through changes in risk factors. As we have seen in this introductory chapter, stress exposure and a lack of social and personal resources might lead to increased levels of depressive symptoms in disadvantaged women. Each of these risk factors has the potential for change and could possibly contribute to the prevention of depression. Therefore, the second part of this dissertation presents studies on the implementation and evaluation of Exercise without Worries, an intervention aims to reduce stress and depressive symptoms in low-SES women living in disadvantaged neighborhoods by means of a combined psycho-education and exercise program.

In Chapter 2 the concept of high-risk groups is addressed. While most disadvantaged women have characteristics known to be associated with depression, not all women in this group necessarily have an identical risk. The purpose of this study was to explore which risk factors are associated with high levels of depressive symptoms in low-SES women and whether there is a question of accumulated risk. Chapter 3 describes a meta-analysis which gives an overview of psycho-social interventions aimed at low-SES women, their effects and possible moderators. Chapter 4 investigates the effectiveness of different strategies for recruiting and retaining low-SES women in the EWW course, and explores which sociodemographic characteristics and risk status factors within this specific target group are associated with their successful recruitment and retention. Chapter 5 describes the theoretical rationale and process evaluation of the EWW, and focuses on the question of whether combined exercise and psycho-education is an acceptable intervention for lowSES women. Chapter 6 provides the results of the effect evaluation of EWW. Short and long term effects on stress and depressive symptoms will be presented as well as moderators of intervention effects. Chapter 7 focuses on the effects of the EWW intervention on several psychosocial outcomes. Finally, Chapter 8 presents a general discussion and conclusion based on the results of 
the previous chapters. Strengths and weaknesses of the studies will be discussed, as will the requisites and obstacles for future implementation of the Exercise without Worries intervention. In addition implications for depression prevention in low-SES women are given, as well as directions for future research in this population. 
1 


\section{Part I}

\section{PREVENTION OF DEPRESSION} IN LOW-SES WOMEN 
26 


\section{Chapter 2}

Which low-SES women from urban neighborhoods are most at risk for depressive symptoms?

Judith E.B. van der Waerden, Cees Hoefnagels and Clemens M.H. Hosman Re-submitted for publication 


\section{Abstract}

\section{Background}

Understanding which risk factors in disadvantaged women contribute most to the development of depression makes it possible to employ targeted screening and offer preventive interventions to subgroups of women who are most at risk. This study explored the relation of demographic, socio-economic and psychological risk factors to the level of depressive symptoms in a sample of low-SES women. Our aim was to examine which factors are associated with self-reported depressive symptoms in this already high-risk population and whether the number of risk factors creates accumulated risk in this population.

\section{Methods}

Between April 2005 and November 2007519 disadvantaged women from urban neighborhoods in Maastricht participated in a cross-sectional survey on stress and depressive symptoms.

\section{Results}

$44 \%$ of the low-SES sample scored above the cut-off score on a depression screening tool. Demographic and community-level SES factors were only marginally associated with depressive symptoms. Lower education levels, no current employment and lower net monthly family incomes were individual level SES factors associated with increased depressive symptoms scores. The psychological risk factor 'perceived stress' had the highest explained variance and was most strongly associated with depressive symptoms. Together all risk factors explained $72 \%$ of the variance in depressive symptoms. Women exposed to multiple risk factors across domains had a cumulated risk for depressive symptomatology.

\section{Conclusions}

Not all disadvantaged women do necessarily have an identical risk for mental health problems. In a population of low-SES women, perceived stress and individual-level SES factors were the strongest predictors of self-reported depressive symptoms. Multiple risk factors notably increased the chance of depressive symptoms in this group. Based on these findings, subpopulations among low-SES women could be targeted with preventive interventions according to their levels of risk. 


\section{Introduction}

Depression is a major mental health problem that contributes to reduced quality of life and increased health care costs (Sobocki, Jönsson, Angst, \& Rehnberg, 2006; Üstün, Ayuso-Mateos, Chatterji, Mathers, \& Murray, 2004). The risk of developing a major depression considerably increases when depressive symptoms are present (Cuijpers, de Graaf, \& van Dorsselaer, 2004; Judd, Schettler, \& Akiskal, 2002). Not all segments of the population are equally affected by this disorder; certain populations suffer the burden of depression more than others. A vulnerable group at high risk for depression is women with low socio-economic status (SES) or living in disadvantaged circumstances (Elliott, 2001; Kuehner, 2003). The relationship between poverty and depression has been studied with low-income women in various life circumstances and across the life span (Groh, 2007). Compared to women from higher socio-economic strata, low-SES women seem to have a more than doubled risk for developing depressive symptoms (ORs between 2.7 and 5.4) (Bassuk, Buckner, Perloff, \& Bassuk, 1998; Kahn, Wise, Kennedy, \& Kawachi, 2000).

Specific life circumstances (i.e. lower levels of education, unemployment, low income and financial strain, and living in disadvantaged neighborhoods), often concomitant with low-SES status, have been found major risk factors for depression (Araya, Lewis, Rojas, \& Fritsch, 2003; Groh, 2007; Klose \& Jacobi, 2004). In women, low education is correlated to higher depressive symptom levels at any given time over the life course (Almeida-Filho et al., 2004). Educational level interacts with current employment status and income, together constituting a substantial social disadvantage (Fryers, Melzer, \& Jenkins, 2003). Low-SES women are more often unemployed or have routine, poorly paid and unfulfilling jobs with low decision latitude and higher job stress (Ross \& Mirowsky, 2006; Sjögren, Kristenson, \& Linquestgroup, 2006). Low household income and financial strain may cause women to live in poorer settings or may perpetuate their living in such areas (Baum, Garofalo, \& Yali, 1999; Belle \& Doucet, 2003). In neighborhoods of concentrated poverty high levels of social disorder and threats to physical safety are often present. Neighborhood disorder has been found to influence depressive symptoms, independent of household income (Schulz et al., 2006).

In the context of socio-economic status, it has been suggested that stress exposure and vulnerability mediate the link between socio-economical disadvantage and depressive symptoms (Belle, 1990; Katerndahl \& Parchman, 2002). Evidence suggests that most of the effects of SES on diminished mental health are potentially attributable to stress (Baum et al., 1999). The relationship 
between stress and risk for depression has been documented for acute and chronic stresses, and for both recent and early negative life events (Liu \& Alloy, 2010). SES may exert some of its effects by increasing the likelihood of exposure to stressful environments and conditions that contribute to chronic stress. Several studies have attempted to assess stress in the lives of disadvantaged women. These studies have shown that in the context of chronic stressful circumstances poor women experience more frequent, more threatening, and more uncontrollable life events than does the general population (Belle, 1990; Ennis, Hobfoll, \& Schroder, 2000). In their turn, depression and depressogenic vulnerability factors can also play an active role in generating the very stresses that place individuals at heightened risk for future depression. Individuals with a history of depression have an increased sensitivity to stress; some studies indicate that this vulnerability to stress is not only related to major life stressors, but minor stressors as well (Ilgen \& Hutchison, 2005). Thus stress generation may to some degree account for the often chronic course of depression (Liu \& Alloy, 2010). Resources, such as coping strategies, social support, a sense of personal control or mastery over one's life, and self-esteem moderate the relationship of individual SES variables to stressors and mental health outcomes (Grzywacz, Almeida, Neupert, \& Ettner, 2004; Katerndahl \& Parchman, 2002). These social and personal characteristics providing resilience to stress are less available to lower status individuals although they still vary within these disadvantaged samples (Denton, Prus, \& Walters, 2004; Elliott, 2001; Piccinelli \& Wilkinson, 2000).

In recent years effective interventions have been developed to enhance resilience and reduce vulnerability to stress and depression in low-SES women (Beeber, Holditch-Davis, Belyea, Funk, \& Canuso, 2004; Peden, Rayens, \& Hall, 2005; Zlotnick, Miller, Pearlstein, Howard, \& Sweeney, 2006). To identify highrisk groups for these targeted prevention programs, risk factors associated with high rates of depressive symptoms are commonly used (Heneghan, Johnson Silver, Bauman, Westbrook, \& Stein, 1998). A particular challenge exists in applying these risk factors to certain high-risk populations in which they may be almost universally present, for instance low-SES women. While most women in this group have characteristics known to be associated with mental health problems, not all disadvantaged women necessarily have an identical risk. Some of them are successfully adapting to the demands or distresses associated with the adverse circumstances they live in and are not in need of preventive or treatment interventions (Weinreb, Perloff, Goldberg, Lessard, \& Hosmer, 2006). Another consideration is that those women exposed to an accumulation of risk factors are probably most at risk of adverse outcomes. A strong relationship has been found between the number of risk factors and 
depressive symptoms in adolescents (Bond, Toumbourou, Thomas, Catalano, \& Patton, 2005) and Almeida et al. (2010) found a similar cumulative probability of prevalent depression according to exposure to risk factors in a sample of older adults. In addition, in their study on disadvantaged Islington mothers, Brown and Moran (1997) showed that especially single mothers experiencing financial hardship had a higher rate of onset of depression, particularly when other proximal risk factors were present. This illustrates that the presence of risk factors my still vary among disadvantaged women

It remains unclear which demographic, socio-economic and psychological risk factors in disadvantaged women contribute most to the development of depression and to what degree these risk factors exert a cumulative effect. A better prediction of which low-SES women are most at risk for depressive symptoms would make it possible to employ targeted screening and offer preventive interventions to subgroups of women who are especially susceptible to mental health problems and most in need of such support. This is especially relevant in a context of limited resources available for preventive interventions. In this study we sought to explore the relation of demographic, socio-economic and psychological risk factors to high levels of depressive symptoms in a sample of low-SES women. Our aim was to examine which factors are associated with self-reported depressive symptoms in this already high-risk population. We also wanted to explore whether perceived stress mediates the relationship between the demographic or socio-economic variables and depressive symptoms and whether the number of risk factors present in the lives of these women might pose them to an accumulated risk.

\section{Method}

\section{Study design}

Between April 2005 and November 2007, community-based epidemiological data on self-reported stress and depressive symptoms were collected in adult (20-55 years) women living in Maastricht, a southern Dutch city. Participants were recruited mainly from the caseloads of general practitioners working in or near the socio-economically deprived neighborhoods. 2501 Women received an invitation from their general practitioner asking them to participate in a short telephone screening about how they felt lately. Women referred by social work and debt repayment services, the district mental health center and Public Health Service, or replying to local media campaigns were also eligible for screening. All women recruited through one of these methods were screened for stress and depressive complaints in a 10-minute telephone interview 
conducted by trained lay interviewers. Approval for conducting this study was provided by the Medical Ethics Committee of the Academic Hospital Maastricht/Maastricht University, the Netherlands.

1383 Women completed the telephone screening. Since our aim for this particular study was to explore risk factors for depressive symptoms among low-SES women, we selected a sub-sample from these women by using years of formal education as a single estimate of socio-economic status. Duration of formal education has shown to be a good indicator of long-term economic position since it often precedes and influences employment, work, earnings, and income, thus acting as a key to positions in the stratification system (Fryers et al., 2003; Ross \& Mirowsky, 2006). Furthermore, it appears that educational level is the socio-economic indicator most strongly linked to mental well-being and common mental disorders (Araya et al., 2003). In the Netherlands, 10 years of education or less is generally considered as the lowest educational attainment (CBS, 2010). Women with less than 10 years of formal education were considered low SES, leading to a sample of 519 women.

\section{Measures}

The screening interview included questions about demographic and socioeconomic characteristics, perceived stress and depressive symptoms. Women's demographic and socio-economic characteristics included age, nationality, marital status, number of children living at home, educational attainment, current occupational situation, and net monthly family income. Women's postal code was used to determine their neighborhood of residence and its associated deprivation score. Neighborhood deprivation is based on a bi-annual community questionnaire, which the town of Maastricht uses to determine overall neighborhood deprivation for each of its districts (Theunissen \& Nijsten, 2007). The neighborhood deprivation score takes into account aspects of population composition (e.g. percentage immigrant and unemployed residents), housing conditions, facilities, safety, social cohesion and responsibility and neighborhood problems. The mean neighborhood score for Maastricht is 10 (range 0 to 21), with more deprived neighborhoods having lower scores.

In addition, women completed the 10-item version of Cohen's Perceived Stress Scale (PSS) (Cohen \& Williamson, 1987). The scale assesses the frequency of stress-inducing situations and feelings of stress over the last month, and was designed for use in the general community. The PSS has an adequate internal and test-retest reliability (Cohen, Kamarck, \& Mermelstein, 1983). Scores range from 0 to 40. Though no standard cut-off score exists for this measure, for screening purposes we used a cut-off of 14 and higher, based on mean PSS 
scores for women in the general population (Cohen \& Williamson, 1987). In the current sample, the test had a Cronbach's $\alpha$ of 0.91 .

Finally, depressive symptoms were assessed using the Center for Epidemiological Studies Depression-Short Form (CESD-SF). This 10-item questionnaire determines the presence of depressive symptoms in the past week, including questions on depressed mood, loss of interest or pleasure in activities, sleep and appetite disturbances and social difficulties. This briefer 10 -item version has a reliability and validity comparable to that reported for the original CES-D. Sensitivity is high (0.85), but specificity is low (0.37) (Cheng \& Chan, 2005). Scores range between 0 and 30, with a cut-off score of 10 for high risk of clinical depression (Andresen, Malmgren, Carter, \& Patrick, 1994). In the current study, Cronbach's $\alpha$ for the total CESD-SF was 0.91 .

\section{Data analysis}

First, the unadjusted associations between demographic, socio-economic and psychological variables with depressive symptoms were examined using Pearson and Spearman correlations. We then performed stepwise linear multiple regression techniques to assess the contribution of several variables to depressive symptoms in our sample. The first model included the demographic variables age, nationality, marital status and number of children living at home. The second model included a community-level SES variable, the neighborhood deprivation score. The third model added individual SES variables: educational attainment, current occupational situation and net monthly family income. The fourth and final model added a psychological variable, perceived stress. Before entering the regression, the initially discrete variables marital status and current occupational status were converted into sets of dichotomous variables by dummy coding. 'Being married or Cohabitating' was used as the reference group in the analysis in the case of marital status and 'Employed' in the case of current occupational status. All other discrete variables were treated as continuous variables.

Second, mediation was assessed, by making a distinction between various effects and their corresponding weights. The total effect (weight $c$ ) of the independent variable (IV) on the dependent variable (DV) is composed of a direct effect (weight $c^{\prime}$ ) of the IV on the DV and an indirect effect (weight $a^{*} b$ ) of the IV on the DV through a proposed mediator $(\mathrm{M})$. Weight $a$ represents the effects of the IV on the M, whereas weight $b$ is the effect of the M on the DV, partialling out the effect of the IV. Depressive symptoms were used as the DV, Perceived stress was used as the $\mathrm{M}$ and each of the demographic and socioeconomic factors was alternatively chosen as the IV, while the remaining variables were treated as covariates. All variables were coded in the same way 
as in the regression analyses. We used a non-parametric bootstrapping approach designed to assess the indirect effect. This bootstrapping procedure assumes that the distribution of the measured variables approximates that of the population while it avoids making the assumption that the indirect effect is distributed normally (Hayes, 2009; Preacher \& Hayes, 2008). According to Preacher and Hayes (2008) and Shrout and Bolger (2002), the bootstrapping procedure is superior to the product of coefficients approach or the Sobel test and the commonly used Baron and Kenny (1986) causal steps approach in terms of statistical power while maintaining reasonable control over Type I error. In this procedure many samples $(n=5000)$ are drawn with replacement from the full data set. Based on the mean of the 5000 estimates, a point estimate of the indirect effect and its $95 \%$ percentile-based confidence interval are calculated. The bootstrap procedure was conducted using the SPSS macro provided by Preacher and Hayes (2008). Mediation is significant if the $95 \%$ bias corrected and accelerated confidence intervals for the indirect effect do not include zero.

Third, to establish whether an accumulation of risk factors might pose an ultra-high risk to some low-SES women, we determined a risk profile for each individual woman based on risk factors found in theory. To this effect, demographic, socio-economic and psychological variables were dichotomized in high versus low risk. High risk was defined as non-Dutch nationality; single, divorced or widowed; $\geq 2$ children living at home; no education, primary school or lower vocational level; housewife, unemployed or disabled; net monthly family income $\leq € 1,100$; neighborhood deprivation score $<10$; PSS score $\geq 17$ (median score). To assess the impact of exposure to multiple risk factors across the different domains, the number of risk factors for which a respondent scored affirmatively was calculated (possible range 0-8). Because much smaller percentages of women endorsed six $(8.1 \%)$, seven $(0.2 \%)$ or eight $(0.2 \%)$ risk factors, these categories were collapsed into one category (six or more risk factors). In a logistic regression model, this cumulative risk score was entered to determine the chance for the different groups to score above the 10-point cut-off for the CESD-SF. We then plotted the probability that women would score above the cut-point of the CESD-SF based on their cumulative risk score. All analyses were performed using PASW Statistics version 17.0.

\section{Results}

Characteristics of the sample are shown in Table 2.1. The mean CESD-SF score was 10.33 (range $0-30 ; S D=8.43$ ) in this sample of low-SES women and 227 
women $(43.7 \%)$ scored above the cut-off score of 10 points, indicating a high risk of clinical depression. Most women were of Dutch nationality and married or cohabitating, $71 \%$ of the sample completed at least lower vocational education, and just over $41 \%$ were currently employed. One third of the women had a net monthly family income of $€ 1,100$ or less, and most women lived in relatively deprived neighborhoods. The mean perceived stress score was 17.19 (range 0-40; SD=9.53), with $296(56.7 \%)$ of the women scoring above the cut-off of 14 points. Table 2.2 presents the correlation coefficients of the variables analyzed in the present study.

Table 2.1 Demographic and socio-economic characteristics and perceived stress in a sample of 519 low-SES women

\begin{tabular}{lc}
\hline & $\%$ or means $(S D)$ \\
\hline CESD-SF score & $10.33(S D=8.43)$ \\
CESD-SF $<10$ & 56.3 \\
CESD-SF $\geq 10$ & 43.7 \\
Age & $43.89(S D=9.29)$ \\
Nationality (\% Dutch) & 93.6 \\
Marital status & \\
Single & 19.1 \\
Married/cohabitation & 72.1 \\
Divorced & 6.6 \\
Widowed & 2.3 \\
Number of children living at home & $0.94(S D=1.03)$ \\
Educational level (\%) & \\
No education & 2.9 \\
Primary school & 16.4 \\
Lower vocational & 51.8 \\
Lower secondary & 28.8 \\
Employment status & \\
Employed & 41.4 \\
Housewife & 28.5 \\
Unemployed & 11.0 \\
Disabled & 19.1 \\
Net family income per month & \\
$€ 0-500$ & 2.3 \\
$€ 500-835$ & 12.7 \\
$€ 835-1100$ & 17.7 \\
$€ 1100-1665$ & 39.3 \\
$€ 1665-2500$ & 19.3 \\
$\geq € 2500$ & 8.7 \\
Neighborhood deprivation score range $0-21$ \\
PSS score & $(S D=4.50)$ \\
&
\end{tabular}


Four models were subsequently examined by multiple linear regression analysis. In the first model incorporating demographic factors and explaining $2.2 \%$ of the variance being single or divorced were significant predictors for higher depressive symptom levels. In a next step, a community SES factor, i.e. neighborhood deprivation, was added. Before doing so, we explored the correlation for depressive symptoms among women in the same neighborhood in order to address the potential correlation between a neighborhood-level variable on an individual-level outcome. The percentage of the variance on depressive symptoms accounted for at the neighborhood level was $2 \%$. When adding the community SES factor to the linear regression model, it's explained variance increased slightly to $3.4 \%$. Being single or divorced, or living in a neighborhood with more deprivation were significant predictors. Adding individual SES variables in the third mode led to a significant increase in explained variance (19\%). Highest educational level, employment status and net monthly family income were significant factors to explain increased depressive symptom levels. When a psychological variable level was added, perceived stress increased the explained variance significantly to $71.8 \%$. In this final model, the individual SES variables 'housewife' or 'disabled' remained significant and together with 'perceived stress' explained increased levels of depressive symptoms (Table 2.3).

To assess the possibility that perceived stress mediates the relationship between the demographic or socio-economic variables and depressive symptoms, mediation analyses were performed. Results based on 5000 bootstrapped samples indicated that community-level SES or demographic factors showed no total or direct effects, or any indirect mediating effects of perceived stress (see Table 2.4). Indirect mediation effects of perceived stress were found for highest educational level, unemployed, and net monthly family income. For each of these indirect effects, zero was not within the $95 \%$ confidence interval, indicating that perceived stress fully mediated the relationship between these variables and depressive symptoms. For the individual SES factors, perceived stress only partially mediated the relation between disabled and depressive symptom scores. Being a housewife remained the only individual-level SES factor not mediated by perceived stress and had a unique direct association with depressive symptom levels.

Finally, a risk profile was created for each woman individually to determine whether an accumulation of risk factors is present among this low-SES population. Women had on average 3.92 risk factors present $(S D=1.28$, range 18). 


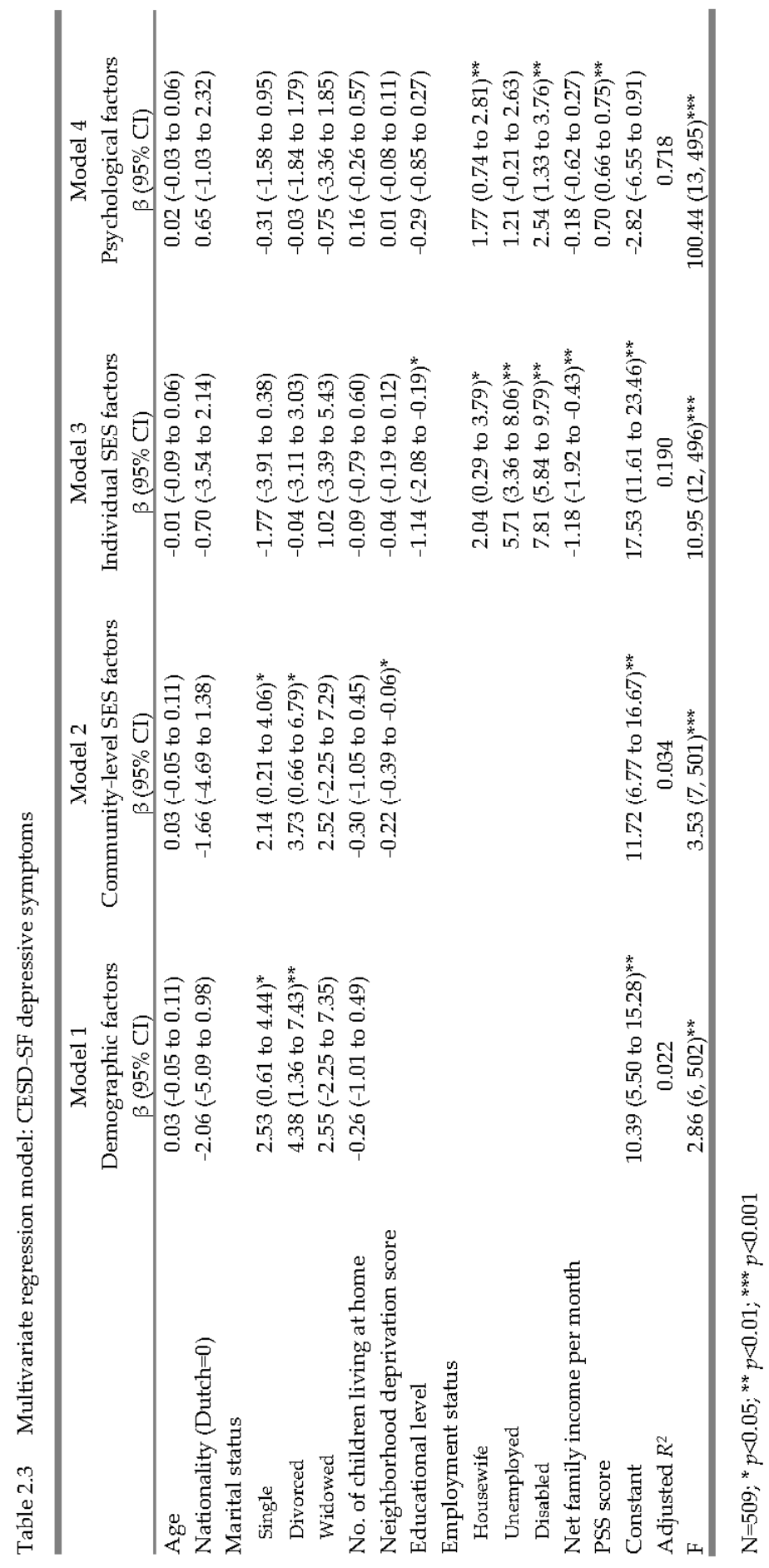




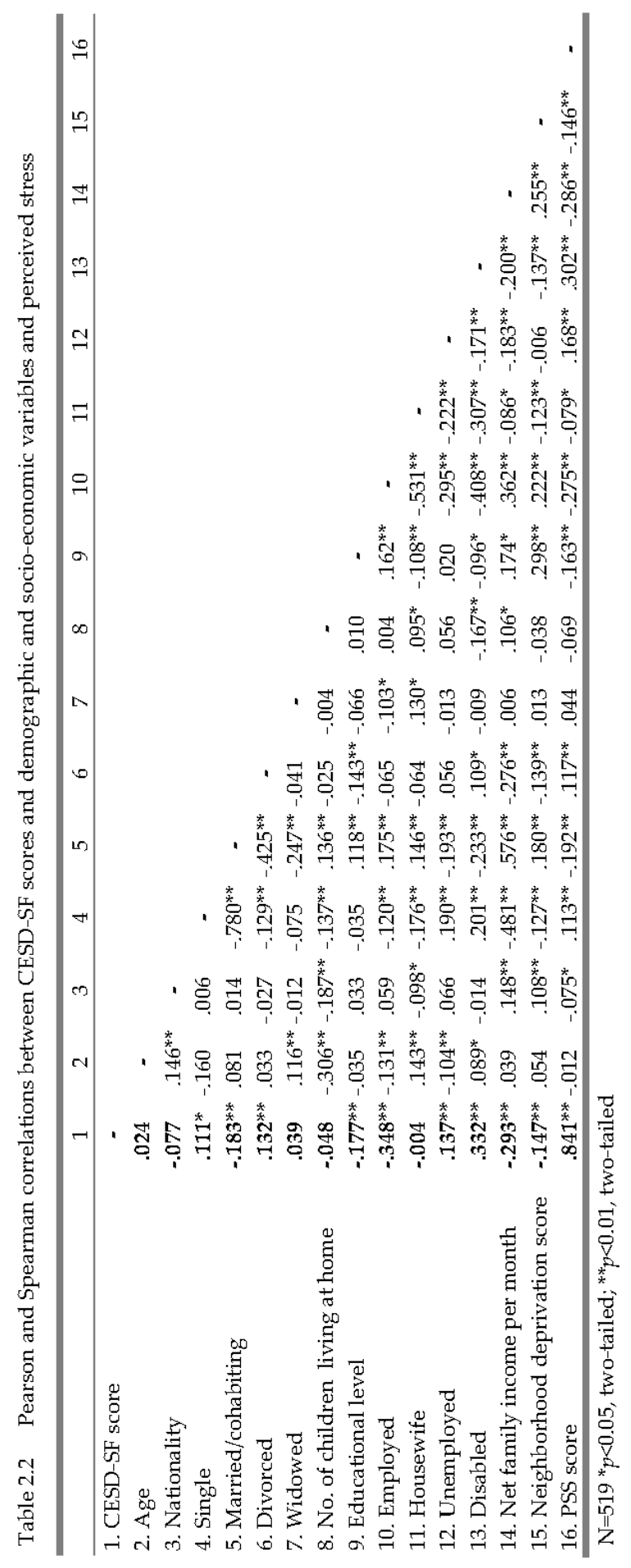




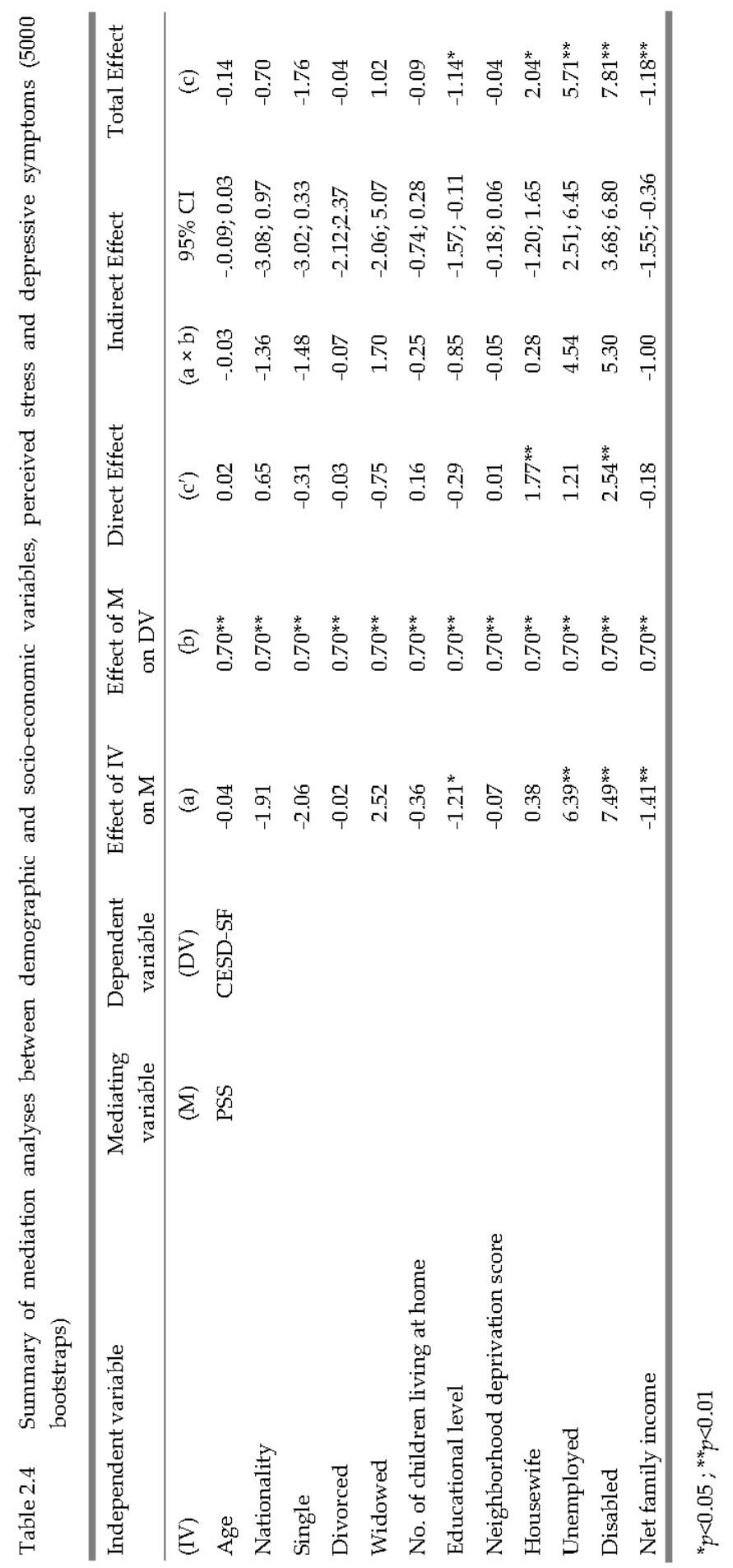


Figure 2.1 depicts the probability of scoring above cut-off ten for high risk of clinical depression in relation to the number of risk factors present. The respondents' probability to score above CESD-SF cut-off increases continuously, starting at $12.5 \%$ for one risk factor and reaching $71 \%$ for women with six or more risk factors present.

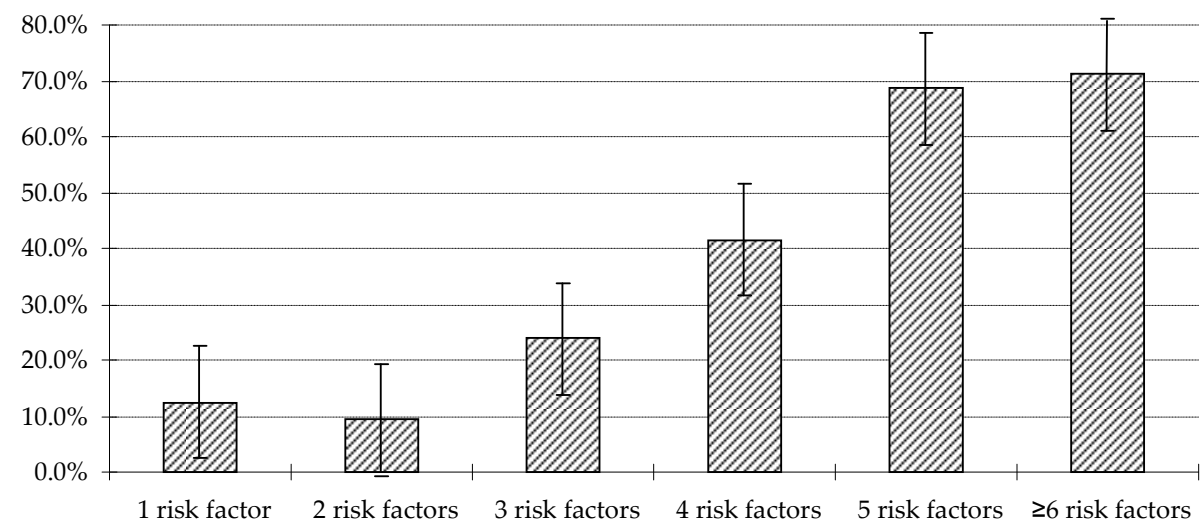

Figure 2.1 Predicted probability of CESD-SF scores $\geq 10$ (\% and CI) and its relation to the presence of risk factors in low-SES women. $N=519$

\section{Discussion}

Individuals with high depressive symptom levels have a consistently greater chance of developing major depression that might be helped with early detection and intervention. This study provided evidence for the importance of a range of demographic, socio-economic and a single psychological risk factor associated with self-reported symptoms of depression in a sample of low-SES women living in urban neighborhoods. While this whole group is often considered to have high risk for depressive symptoms, approximately $56 \%$ of our low-SES sample scored below the cut-off score on a depression-screening instrument. This indicates that not all disadvantaged women are at an equally high risk of clinical depression. Together, the outcomes suggest that it is possible to indicate which women in this high-risk group have the most depressive symptoms. Especially perceived stress was associated with depressive symptoms. Perceived stress itself contributes not only to increased level of depressive symptoms, but it also mediates some of the individual-level SES factors that may contribute to depressive symptomatology. While 
exposure to only one risk factor may not put low-SES women at elevated risk for depressive symptoms, this study showed that the cumulative effect of multiple risk factors notably increased the chance for depressive symptoms. This finding is especially important since it might help to explain why certain low-SES women experience fewer mental health problems than others.

Findings from this study corroborate with previously reported outcomes on the marginal association of demographic factors with depressive symptoms in low-SES populations (Fleischer, Fernald, \& Hubbard, 2007; Hamad, Fernald, Karlan, \& Zinman, 2008; Heneghan et al., 1998). We did find a statically significant association between community-level SES and depressive symptoms, but this effect disappeared when individual-level SES factors were added to the model. While this outcome is in line with some studies that found no link between neighborhood characteristics and depression once individual and family socio-economic factors were taken into account (Henderson et al., 2005), other authors did find a contribution of adverse neighborhood circumstance to depression above and beyond the effects of these individual factors (Cutrona, Wallace, \& Wesner, 2006; Matheson et al., 2006). In their systematic review Mair et al. (2008) concluded that the associations of depressive symptoms with structural neighborhood features were less consistent than with social processes.

Our findings concerning the individual-level socio-economic correlates of depressive symptoms confirmed outcomes of previous research among lowincome adults (Araya et al., 2003; Hamad et al., 2008). Although most low-SES women in this sample have characteristics known to be associated with mental health problems, we found that especially those with the lowest education levels, no current employment and lower net monthly family incomes showed high depressive symptoms scores.

Psychological risk factors such as perceived stress are generally the variables most strongly associated with depressive symptoms (Fleischer et al., 2007; Heneghan et al., 1998). In our sample, perceived stress contributed most to the explained variance for depressive symptoms and also mediated the effects of some individual-level SES factors. The PSS and CESD-SF scales showed a strong mutual correlation. One interpretation might be that there is some overlap between the two respective constructs as being measured by both instruments, since perception of stress may be a symptom of depression. However, Cohen et al have indicated that albeit the high correlation with the CES-D scale, the PSS is able to prospectively predict psychological symptoms when taking this common relation into account (Cohen et al., 1983). While high scores on the PSS are not the same thing as elevated depressive symptom 
scores, they do place people at risk for future distress (Cohen \& Williamson, 1987). In the context of our sample, this perceived stress may reflect the chronic stressful conditions in the lives of most disadvantaged women. Women with lower educational levels, who are unemployed or disabled or have lower monthly family income are more likely to experience feelings of stress, which in turn is associated with more depressive symptoms.

The individual-level SES factors of being a housewife and disabled working status remained significant in the final model. It might be that disabled status was not completely mediated by perceived stress as it can be seen as a proxy indicator for poor physical health or chronic conditions, both of which are strongly associated with depressive symptoms as well (Cassem, 1995). Being a housewife was the only individual-level SES factor whose association with depressive symptom levels was not mediated by perceived stress. While several studies have shown that housewives generally experience more depressive symptoms (Aro, Nyberg, Absetz, Hendriksson, \& Lönnqvist, 2001; Klose \& Jacobi, 2004), the mechanisms which influence this association remain unclear. A possible pathway may be the relative social isolation of housewives leading to higher depression scores (Fleischer et al., 2007). Given our finding that perceived stress seems to be the a factor that mediates the association between several other risk factors and depressive symptoms, depression prevention strategies may benefit from focusing on stress management or emotion regulation techniques. To our knowledge, only a few interventions (e.g. Baxter \& Kahn, 1999; Peden et al., 2005) designed specifically to focus on stress management for low-SES groups are currently available.

We also sought to determine if findings from other populations, in which an accumulation of risk factors was shown to increase the likelihood of depressive symptoms, were also present for this low-SES population. Probably one of the most important findings from this study is that multiple risk factors notably increased the chance of depressive symptoms in this population. This finding is especially important since it might help to explain why some low-SES women experience fewer mental health problems than others. For instance, having a low educational level does not necessarily lead to depression, but if a woman is simultaneously unemployed, has several children living at home and has to manage a low family income her risk for depression increases. It can be argued that disadvantaged women most eligible for preventive strategies are those with an increased number of risk factors. The cumulative risk score can serve as a simple and practical tool that allows practitioners or public health workers to quickly identify women at highest risk for depression. Most demographic or socio-economic risk factors can be identified by asking a few simple questions to women in these neighborhoods (e.g. on financial situation, marital status), 
which require no extra investment in time or money. Since perceived stress levels seem to be the best predictor of depression, their presence could be determined as well, which can be relatively easily done. Previous studies (Baas et al., 2009) have shown that screening general high-risk groups in general practice populations leads to the detection of a limited number of persons with elevated complaints. Targeting those low-SES women with an elevated number of risk factors will probably increase the success rates of depression screening. This targeted selection of women in psychological need will benefit from prevention or treatment of depression among disadvantaged women.

The primary limitation of this study is the cross-sectional nature of the design. This limits the possibility to make causal inferences linking depressive symptoms to the presence of risk factors or to test the direction of the effects. Further, we used years of formal education as a single estimate of socioeconomic status for selection of our sample. Although it has been found that education as a measure for socio-economic disadvantage is a better predictor of depression in women than in men (Van de Velde, Bracke, \& Levecque, 2010) it may have been possible that by using this measure we could have, for instance, excluded those women with a high educational level, but who are unemployed or have low monthly incomes. Repeating our analyses with net monthly family income as proxy indicator of low-SES (data not shown), showed outcomes consistent with presented findings, increasing confidence in the validity of our results.

Another limitation may be that we did not include other specific risk factors for depression such as lack of social support or chronic health conditions (Aro et al., 2001; Fleischer et al., 2007). These factors could possibly have added to our understanding of some of the outcomes, such as the contribution of the assumed social isolation among housewives to the risk of depression.

Finally, it should be born in mind that the risk profile found in this research cannot be automatically applied to other low-SES populations. For instance, ethnic minorities are relatively underrepresented in the overall population of Maastricht and thus in our sample. The risk profile for a more ethnically diverse population could be different from the profile presented in this study. As an example, while African Americans are often exposed to greater social disadvantages than whites, they experience a lower burden of stress-related psychopathology (Mezuk et al., 2010). In contrast, they engage more in poor health behaviors as a stress-coping strategy resulting in a higher prevalence of physical health problems (Jackson, Knight, \& Rafferty, 2010). 
Despite these constraints, we can conclude that this study contributes to a better awareness of factors related to depressive symptoms in a high-risk group of disadvantaged women residing in urban neighborhoods. In this population we have indicated those risk factors that were the strongest predictors of self-reported depressive symptoms, making it possible to identify those low-SES women who are most at risk. While several individual-level SES indicators appear to be important, women who seem most eligible for targeted preventive action are those with elevated perceived stress levels. Furthermore, those women exposed to multiple risk factors across various domains have an accumulated risk for depressive symptomatology. Since budget and staff for prevention are often restricted, focusing on these women might help to increase efficiency and devote limited resources to those populations most in need. 


\section{Chapter 3}

Psychosocial preventive interventions to reduce depressive symptoms in low-SES women at risk: A meta-analysis

Judith E.B. van der Waerden, Cees Hoefnagels and Clemens M.H. Hosman Journal of Affective Disorders 2011;128:10-23 


\section{Abstract}

\section{Background}

Women who have low socioeconomic status (SES) or live in disadvantaged circumstances are a vulnerable group at risk for depression. Little is known about the efficacy of preventive interventions to reduce depressive symptoms in low-SES women. The aim of this study is to provide an overview of controlled outcome studies and to investigate the overall efficacy and moderators of interventions targeted at reducing depressive symptoms in this population.

\section{Methods}

A systematic review and meta-analysis were conducted for 14 studies $(N=1396)$. The effect size of the studies was computed for outcomes assessing changes in depressive symptom levels using the standardized mean difference effect size. Study, target population, and intervention descriptors expected to influence effect size were analyzed using univariate subgroup and metaregression techniques with mixed-effects statistical models.

\section{Results}

The estimated overall effect size of 0.31 was significant; study sample characteristics, intervention characteristics and the research design of the studies did not moderate intervention effects.

\section{Conclusions}

A number of promising programs have been developed specifically for low-SES women, a population at high risk for developing major depression. On average these programs were found to reduce the level of depressive symptoms, with more than half of the studies showing medium to large effect sizes. This indicates that considerable mental health benefits can be gained among disadvantaged women. 


\section{Introduction}

Depressive disorder is a major mental health problem in the general population, with 12-month prevalence rates of approximately 5 to $7 \%$ (Demyttenaere et al., 2004). The presence of depressive symptoms considerably increases the risk of developing a major depression (Cuijpers, de Graaf, \& van Dorsselaer, 2004; Judd, Schettler, \& Akiskal, 2002). Research has shown that women and people with low socioeconomic status (SES) appear to be especially affected. Incidence and prevalence rates of depressive disorders are higher among women than men, with a sex ratio of about 2:1 (Kuehner, 2003; Seedat et al., 2009). Studies show a similar pattern for low SES (Dohrenwend, Levav, Shrout, \& Schwartz, 1992; Gutierrez-Lobo, Wölfl, Scherer, Anderer, \& Schmidl-Mohl, 2000), with odds ratios (ORs) of 1.81 for depression prevalence and 2.06 for depression persistence in the lowest SES group (Lorant et al., 2003). Thus, women who have low SES or live in disadvantaged circumstances emerge as a particularly vulnerable group at risk for depression (Elliott, 2001; Kuehner, 2003). Compared to women with higher SES, low-SES women seem to have a more than doubled risk for developing depressive symptoms (OR 2.7) (Kahn, Wise, Kennedy, \& Kawachi, 2000). Similarly, for single-parent families, often headed by women, an OR of 3.37 has been found for the prevalence of mood disorders in the past 12 months (Bijl, Ravelli, \& van Zessen, 1998).

Numerous factors may contribute to depression in women, including hormonal, genetic, and other biological differences. In the aetiology of depression, stress factors also play an important role (Hauenstein, 1996a). The number and types of stressors as well as the mechanisms for coping with stress may all contribute to an adverse outcome. Chronic stress and daily hassles in particular are considered strong predictors of depressive episodes and symptoms (Hammen, 2003; McConagle \& Kessler, 1990). Low-SES women may have the same biological vulnerability for depression as other women, but are more likely to suffer from depression as a result of elevated exposure to stressors, which is socially patterned by socioeconomic status (Denton, Prus, \& Walters, 2004; Sterk, Theall, \& Elifson, 2006). Disadvantaged women are often exposed to an accumulation of life-event stressors and chronic problems related to their low SES status, such as poor education, poor labour circumstances, and unemployment (Brown, Bifulco, \& Harris, 1987; Elliott, 2001), and financial strain, inadequate housing, and neighbourhood violence (Ennis, Hobfoll, \& Schroder, 2000; Peden, Rayens, Hall, \& Grant, 2004). The presence of chronic stressors may directly influence psychological distress, but may also indirectly lead to an increase in the number of acute stressors that 
low-SES women consequently have to deal with (Ennis et al., 2000; Hammen, 2003).

The vulnerability of disadvantaged women to the negative effects of stressful events (e.g. the development of depression), is further increased by their lack of psychological resources to limit the harmful impact of stressful conditions or minimize depressive symptoms. Lower self-esteem, lower perceived control or mastery over their lives are some examples of vulnerabilities that decrease the likelihood that low-SES women will seek and sustain adequate efforts toward solving problems (Ennis et al., 2000; Gallo, Bogart, Vranceanu, \& Matthews, 2005; Nolen-Hoeksema, Larson, \& Grayson, 1999). The chronicity of major depression, its impairing impact on role functioning, and high rate in disadvantaged women thus create a significant public health problem (Hauenstein, 1996b).

In recent years more attention has been paid to the treatment of mental health problems in this group. A brief overview of the literature shows that several effective depression therapies have been developed and adapted to the needs of low-SES women from different cultural backgrounds.

For example, a controlled clinical trial offering interpersonal psychotherapy treatment to disadvantaged women with antepartum depression showed significant improvement compared to a parenting education control program. At termination, $60 \%$ of those in the IPT group recovered, whereas only $15 \%$ of those receiving parent education did so (Spinelli \& Endicott, 2003). In addition, Miranda and colleagues (2003) examined the impact of cognitive behavioral psychotherapy and antidepressant medication on working poor women. The interventions were modified to be sensitive to low-income women, and separate modest cultural adaptations were made for Latina and African American women. Both the medication intervention and the psychotherapy intervention were effective over and above community care in decreasing depressive symptoms and improving functioning of these women, even after one year follow-up (Miranda et al., 2006). Similarly, Kohn et al (2002) conducted an evaluation of a CBT intervention adapted to be culturally sensitive in treating depressed low-income African American women. Following treatment, women in the adapted intervention exhibited a larger drop in depressive symptoms scores than women treated in a non-adapted intervention. Finally, a study in Santiago, Chile compared the effectiveness of a stepped-care program with usual care in primary-care management of depression in low-income women. The stepped care comprised a 3-month, multicomponent intervention including a psychoeducational group intervention, structured follow-up, and drug treatment for patients with severe depression. At six-month follow-up, 70\% of the stepped-care group, compared 
with $30 \%$ of the usual-care group, was no longer symptomatic (Araya et al., 2003). Thus in general it seems that well-established psychotherapies such as in Cognitive Behavioral Therapy, Interpersonal Therapy and antidepressant medication are effective for disadvantaged women.

Despite advances in treating depression in low-SES women, the extent to which this group is confronted with depression and their underutilization of mental health services (Katon \& Ludman, 2003; Mojtabai, 2005) calls for additional approaches to reduce mental health disparities. One approach to lower the severity and incidence depression among low-SES women would be to shift attention from treatment to prevention. Increasing evidence supports the primary prevention of depression (Dennis, 2005; Mendelson \& Muñoz, 2006) and several randomized controlled studies have shown that preventive interventions aimed at participants with an elevated level of depressive symptoms may reduce their risk for developing a depressive disorder (Clarke et al., 2001; (Willemse, Smit, Cuijpers, \& Tiemens, 2004). However, less is known about the efficacy of preventive interventions specifically designed for and targeted at one of the populations most at risk: low-SES women. These interventions are usually adapted to fit the needs, competences and specific risk factors of disadvantaged women and can produce outcomes that enhance their resilience and reduce their vulnerability to depression. Research has found improved outcomes in both treatment and prevention efforts for depression when interventions take participants' social and cultural contexts into account (Podorefsky, McDonald- Dowdell, \& Beardslee, 2001).

Essential for understanding intervention effects and improving program outcomes is knowledge and comprehension of potential outcome moderators in the study population, characteristics of the intervention and the research design of the studies. Previous meta-analyses (Cuijpers, Van Straten, Warmerdam, \& Smits, 2008; Jané- Llopis, Hosman, Jenkins, \& Anderson, 2003) identified outcome moderators for depression prevention interventions. Important moderating variables appeared to be program type and delivery mode, duration and timing of the intervention and type of intervention provider. Study variables that showed a moderating effect were both study quality and type of comparison group. However, it is unclear whether these moderators are also valid for interventions targeted at low-SES women.

This meta-analysis focuses on interventions specially targeting the selective or indicated prevention of depressive symptoms in low-SES women. The first aim is to provide an overview of preventive interventions specifically designed to reduce depressive symptoms in low-SES women and to examine the overall efficacy of these interventions, both immediate and over time. The second aim is to conduct an exploratory moderator analysis to systematically examine 
possible intervention, research design, and sample characteristics that may be associated with larger effects. By analyzing the effects of specific interventions for this population, this meta-analysis aims to contribute to the primary prevention of depression in low-SES women by identifying potentially influential intervention features.

\section{Method}

\section{Search strategy}

Relevant studies were identified by searching the PubMed database of the National Library of Medicine, the PsycINFO and ERIC databases, and the Web of Science. The search strategy combined the following three groups of keywords: depress*, depressive symptoms, minor depression, psychological distress; social class, disadvantaged, poverty, socioeconomic status, SES, financial strain; prevent*, intervention, program*, early treatment and mental health services. Searches were specified for both $\mathrm{MeSH}$ terms, and text words and were modified to meet the requirements of each database. We selected articles in English, French, German, and Dutch to avoid possible bias due to too-strict language criteria, published between January 1995 and April 2009. Two reviewers independently inspected the reference titles and abstracts retrieved via the database search to evaluate their potential eligibility. The original articles of relevant abstracts were then retrieved for further evaluation. We scanned the reference lists of identified articles and also contacted experts in the field to find relevant publications.

\section{Inclusion criteria}

To be selected, the studies had to be randomized controlled trials or quasiexperimental studies with one comparison group consisting of treatment as usual or a different intervention. The participants had to be adult low-SES women of any ethnicity. To meet low-SES inclusion criteria, the study authors had to have reported that more than $70 \%$ of the study participants were living in economically deprived conditions (e.g., depending on welfare, living under the poverty line, or belonging to the lowest income category), had low educational achievements (twelve years of formal education or less) or were currently unemployed.

The interventions had to be aimed at selective or indicated depression prevention and could include psychological, social or physical interventions, or policy actions. To meet the criterion of selective or indicated prevention, the 
authors had to have indicated that they were aiming their intervention at groups of high-risk women or including women with elevated levels of depressive symptoms. Studies that explicitly reported aiming the intervention at women with depressive disorders were excluded. However, when a preventive intervention does not determine absence of depressive disorder at baseline by way of a clinical instrument but rather uses self-report questionnaires only, the possibility that some women met the criteria of a MDD at time of registering for the intervention cannot be excluded. If that were the case, the intervention would no longer be focusing on primary prevention, but rather on depression treatment (Mrazek \& Haggerty, 1994). To maximize the inclusion of studies aimed at low-SES women, we included studies that did not use a diagnostic instrument to determine baseline depression caseness, and counted the use of a diagnostic instrument as a moderator in the analyses. Finally, for a study to be included its principal outcome measures had to be aimed at measuring depressive symptom levels.

\section{Study quality}

Poor study design and lack of rigor in its implementation can result in biased effect estimates (Khan, Daya, \& Jadad, 1996; Moher et al., 1998). To evaluate study quality we appraised each study on methodological criteria based on the Agency for Healthcare Research and Quality (AHRQ) domains for RCTs (West et al., 2002). The quality coding list included aspects of study design such as description of study population, random assignment to conditions, concealment of treatment allocation, use of intention to treat analysis and reported dropout and follow-up data. Furthermore, we assessed whether the study itself was adequately described (description of intervention, reference to procedures) and appropriate statistical analyses were used (procedures, reliability, and validity of measures). The higher the score a study received, the stronger its methodological quality, and thus the internal validity of the reported effects. If a criterion was only partly met, half a point was credited. In total, a study could receive 14 points at most. All were independently rated on study quality by the first author and a $\mathrm{PhD}$ candidate in prevention research (Cohen's kappa 0.68, interrater agreement 85\%). Differences in observations were discussed to reach full agreement on the final score.

\section{Data extraction}

The first author and a trained coder independently assessed the studies not only on quality, but also on participant and intervention information and main study outcomes. A coding sheet was developed to guide the evaluation 
process. This included variables for trial descriptors, target group characteristics, program characteristics, implementation characteristics, and outcome indicators. Incongruities between coders were discussed and checked against the original article to reach agreement.

\section{Data analysis}

Effect sizes for the intervention effects on depressive symptom levels were calculated using the standardized mean difference effect size, which refers to the difference between the program and the comparison groups relative to the standard deviation of the measure used. Outcome codes were reassigned where needed such that a positive effect size indicated improvement in the intervention group, whereas a negative effect size indicated poorer outcomes for the intervention group compared to the comparison group. When a study used more than one outcome measure for depressive symptoms, the effect sizes for multiple measures were standardized and then averaged to calculate a single effect size per study (Gleser \& Olkin, 1994). Thus, each study provided the meta-analysis with only one effect size to avoid statistical dependency between effect sizes. To this same end, when a study examined the effects of more than one intervention to a comparison condition, we considered only one of the intervention conditions in the analyses. This was the case for 1 study (Wiggins et al., 2005; Wiggins et al., 2004), in which the active intervention, i.e. the intervention the authors assumed to result in preventive effects, was included for the meta-analysis

Given that the standardized mean difference has been proven to be higher in small sample sizes, effect sizes were adjusted to correct for bias (Hedges's $g$ ), in line with Hedges and Olkin (1985). To combine effect sizes from different studies into a weighted mean effect size, each effect size was weighted by the inverse of its variance and then averaged (Lipsey \& Wilson, 2001). Confidence intervals of 95\% were calculated in the vein of Hedges and Olkin (1985) to indicate the degree of precision of the mean effect size estimate. To test for homogeneity, the Q statistic was computed to determine whether each set of effect sizes shared a common population effect size (Lipsey \& Wilson, 2001). The $\mathrm{I}^{2}$ statistic, equal to the percentage of total variation across the studies due to heterogeneity, was used to aid the interpretability of between-study heterogeneity (Higgins et al., 2003).

The influence of moderators on the set of overall mean effect size was assessed with univariate subgroup and metaregression analyses using mixed effects models (Raudenbush, 1994). These models assume that variability in effect size distribution is due to systematic between-study differences, subjectlevel sampling error, and an additional random component (Lipsey \& Wilson, 
2001). We investigated potential sources of heterogeneity in the study sample characteristics, intervention characteristics and the research design of the studies themselves.

Mean sample age, marital status and pre or postpartum timing were used as sample moderators. We have selected these sample characteristics since the literature showed that especially women who are younger or heading singleparent families have a higher risk for depression (Azocar, Miranda, \& Dwyer, 1996; Gutierrez-Lobo et al., 2000). Since a considerable number of studies are aimed at women during or after their pregnancy, pre of postpartum timing was considered as a moderator as well. In line with the meta-analyses from Cuijpers et al. (2008) and Jané Llopis et al. (2003), included intervention moderators were main program type, intervention delivery mode, program setting, intervention providers, intervention duration in weeks and presence of booster sessions. Study design moderators were used to examine potential threats to the validity of this meta-analysis and include type of comparison group, use of a diagnostic instrument to determine baseline depression caseness and study quality. Specifications of these moderators are included in Table 3.2. A separate meta-analysis was performed on follow-up assessments, reporting these effect sizes separately from the immediate post-assessment to explore deterioration or gain of intervention effects over time.

The results of a meta-analysis may be biased because studies with nonsignificant results are less likely to be published than those with significant results. To detect publication bias, a funnel plot was examined both graphically and using Egger's linear regression method (Egger, Davey Smith, Schneider, \& Minder, 1997). Furthermore, failsafe $\mathrm{N}$ was calculated to assess the robustness of the findings obtained in relation to the possibility that not all relevant studies were retrieved. All meta-analyses were conducted using Statistical Package for the Social Sciences version 15.0.

\section{Results}

\section{Characteristics of included studies}

Searches of the electronic databases, manual searching and expert contacts identified 4953 titles. After screening of identified titles, 199 abstracts were further inspected to identify potentially eligible studies. Abstracts were screened by the primary author, and the second author independently rated a random sample of $25 \%$. Interrater agreement on inclusion or exclusion of abstracts was $95 \%$. A total of 111 articles were retrieved for further full-text evaluation on the inclusion criteria. After this detailed inspection, 18 articles 
reporting short- or long-term outcomes for 14 different studies met the inclusion criteria. We contacted eight authors for further details on their interventions, and five provided them. Figure 3.1 presents a flowchart of this selection process.

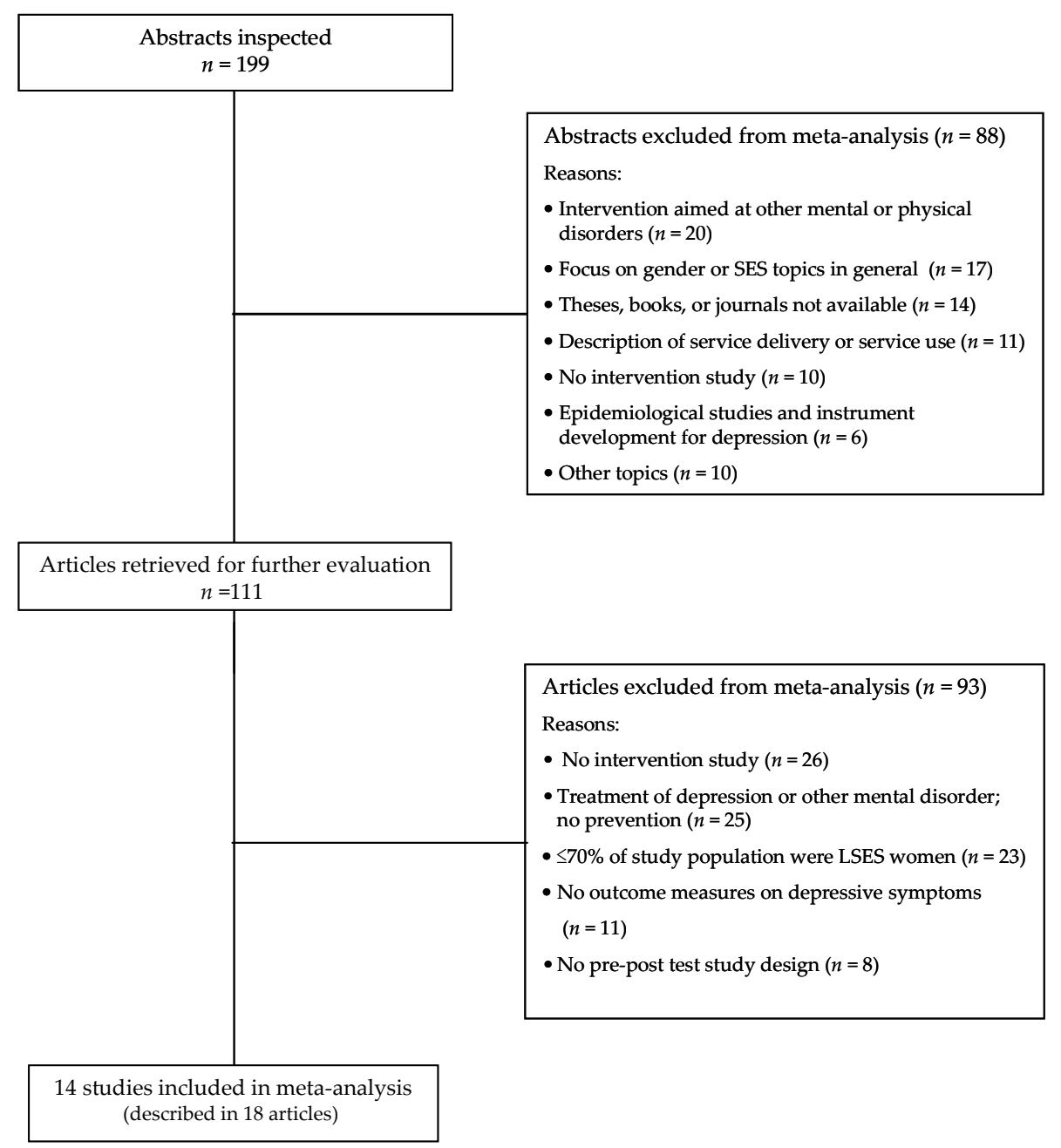

Figure 3.1 Selection process for studies included in meta-analysis.

The 14 studies included in this review covered a total of 1396 participants. Three studies were conducted in non-English-speaking countries, namely 
Mexico (Lara, Navarro, Rubi, \& Mondragon, 2003a), Pakistan (Ali et al., 2003), and Turkey (Tezel \& Gözüm, 2006). Ten interventions explicitly indicate to be designed for or adapted to meet the needs of low-SES women. These adaptations mostly entailed intervention contents paying attention to the specific problems of this target group and easier comprehension levels of the material or exercises. For four studies (Ali et al., 2003; Laperriere et al., 2005; Peden, Rayens, \& Hall, 2005a 2005b; Tezel \& Gözüm, 2006) it was not clear whether the intervention was developed or adapted for disadvantaged women or delivered to this population without specific modifications. Table 3.1 provides an additional description of the various preventive interventions employed by the included studies. The mean age for all participants was 27.9 $(S D=4.88)$. Six of the interventions (Bullock, Wells, Duff, \& Hornblow, 1995; Crockett, Zlotnick, Davis, Payne, \& Washington, 2008; Muñoz et al., 2007; Tezel \& Gözüm, 2006; Zlotnick, Johnson, Miller, Pearlstein, \& Howard, 2001; Zlotnick, Miller, Pearlstein, Howard, \& Sweeney, 2006) focused on preventing depression in the pre- or postpartum period, while four others (Beeber, Holditch-Davis, Belyea, Funk, \& Canuso, 2004; Lipman \& Boyle, 2005; Peden et al., 2005; Wiggins et al., 2004) aimed to support women with children and often focused both on mother and on child outcomes.The commonest main preventive intervention type was psycho-education, which proposes educational interventions through the application of cognitive-behavioural mechanisms, stress-management techniques or counselling activities. Interpersonal therapy was used in four interventions (Beeber et al., 2004; Crockett et al., 2008; Zlotnick et al., 2001; Zlotnick et al., 2006), while three others used social support (Bullock et al., 1995; Lipman \& Boyle, 2005; Wiggins et al., 2004).

The intervention delivery format was generally in the form of group courses. However, five interventions (Ali et al., 2003; Beeber et al., 2004; Bullock et al., 1995; Tezel \& Gözüm, 2006; Wiggins et al., 2004) were offered through individual in-home sessions or telephone support (Bullock et al., 1995). In these cases the intervention provider would have played a more extensive role in the intervention delivery. In $56 \%$ of the studies, the interventions were delivered by mental health professionals, such as psychologists, psychiatric nurses or counsellors. Nurses and trained nonprofessionals were each used in $22 \%$ of the studies. Intervention duration varied greatly, from 4 to 52 weeks $(M=13$ weeks, $S D=12.78)$. Only four studies (Beeber et al., 2004; Crockett et al., 2008; Muñoz et al., 2007; Zlotnick et al., 2001) provided booster sessions at the end of the intervention period. 
56 Chapter 3

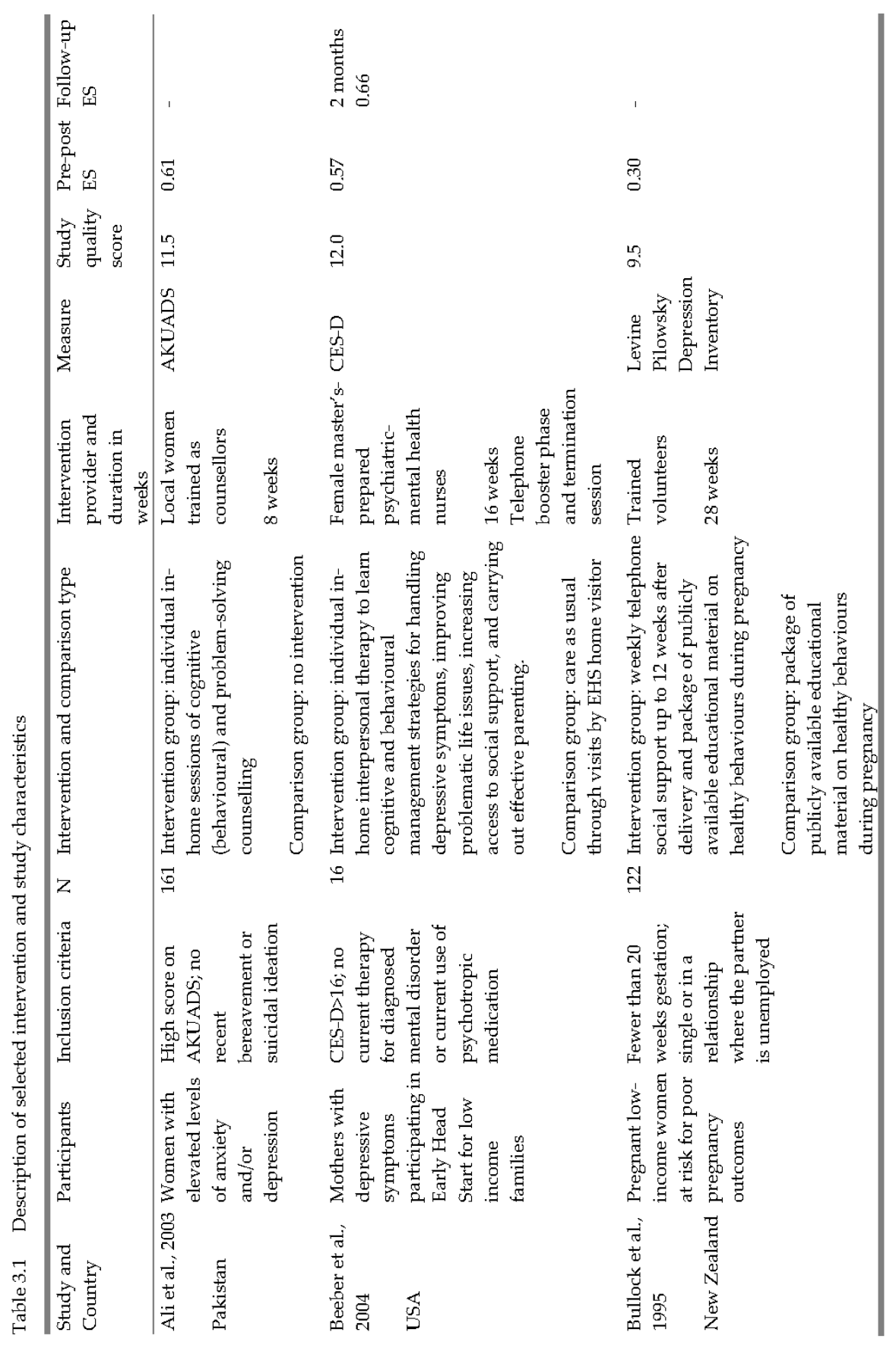




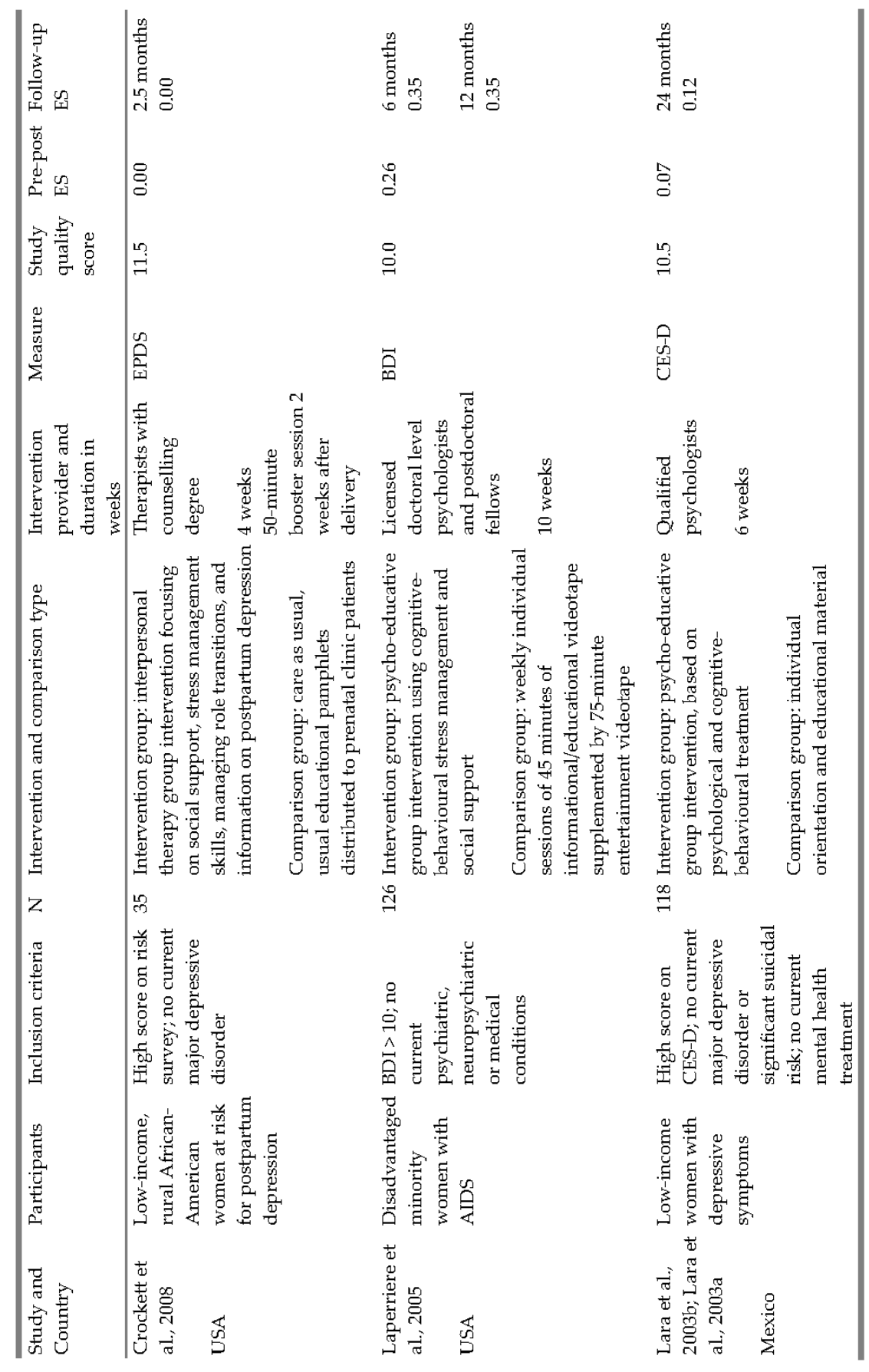


58 Chapter 3

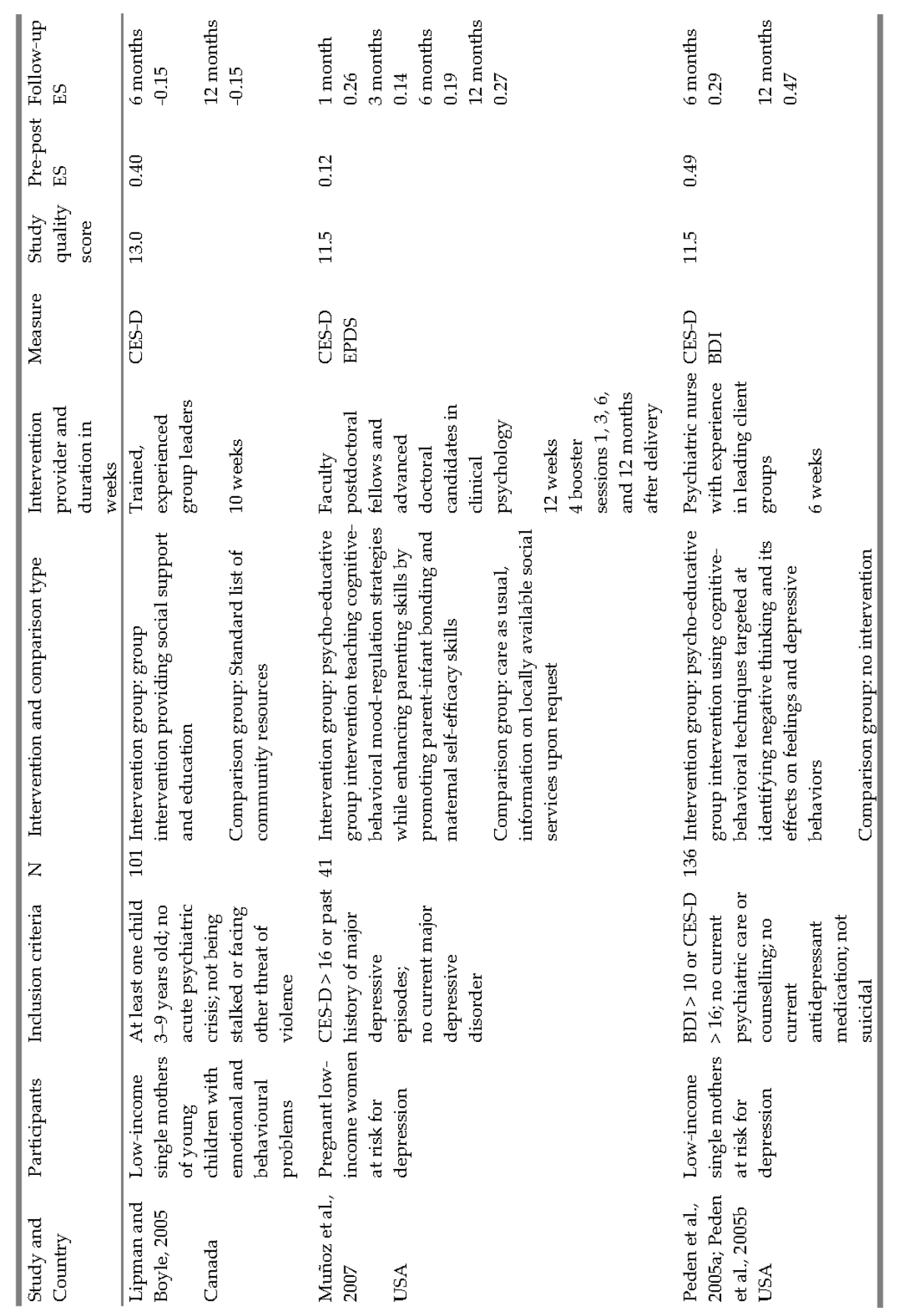




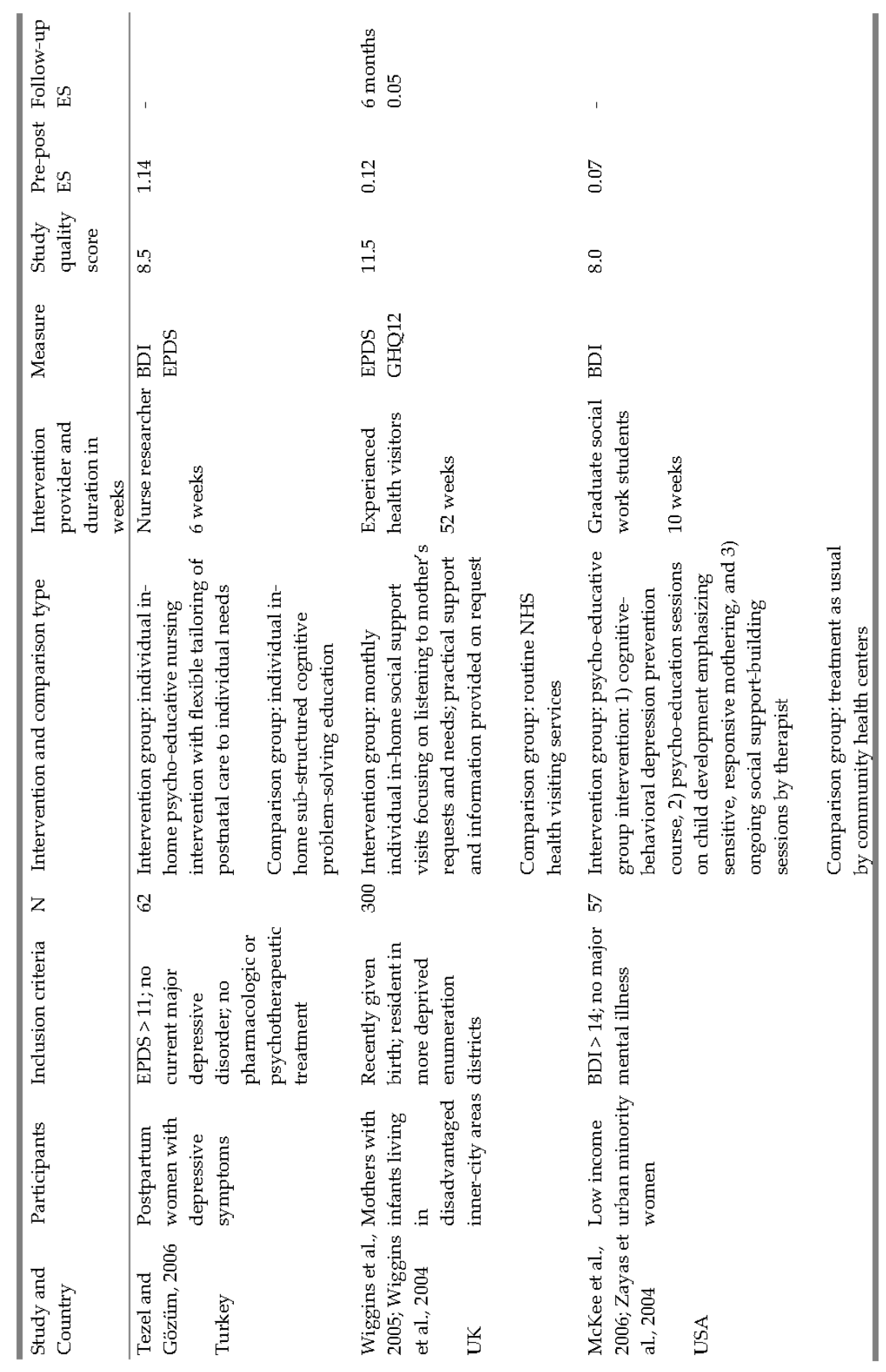


60 Chapter 3

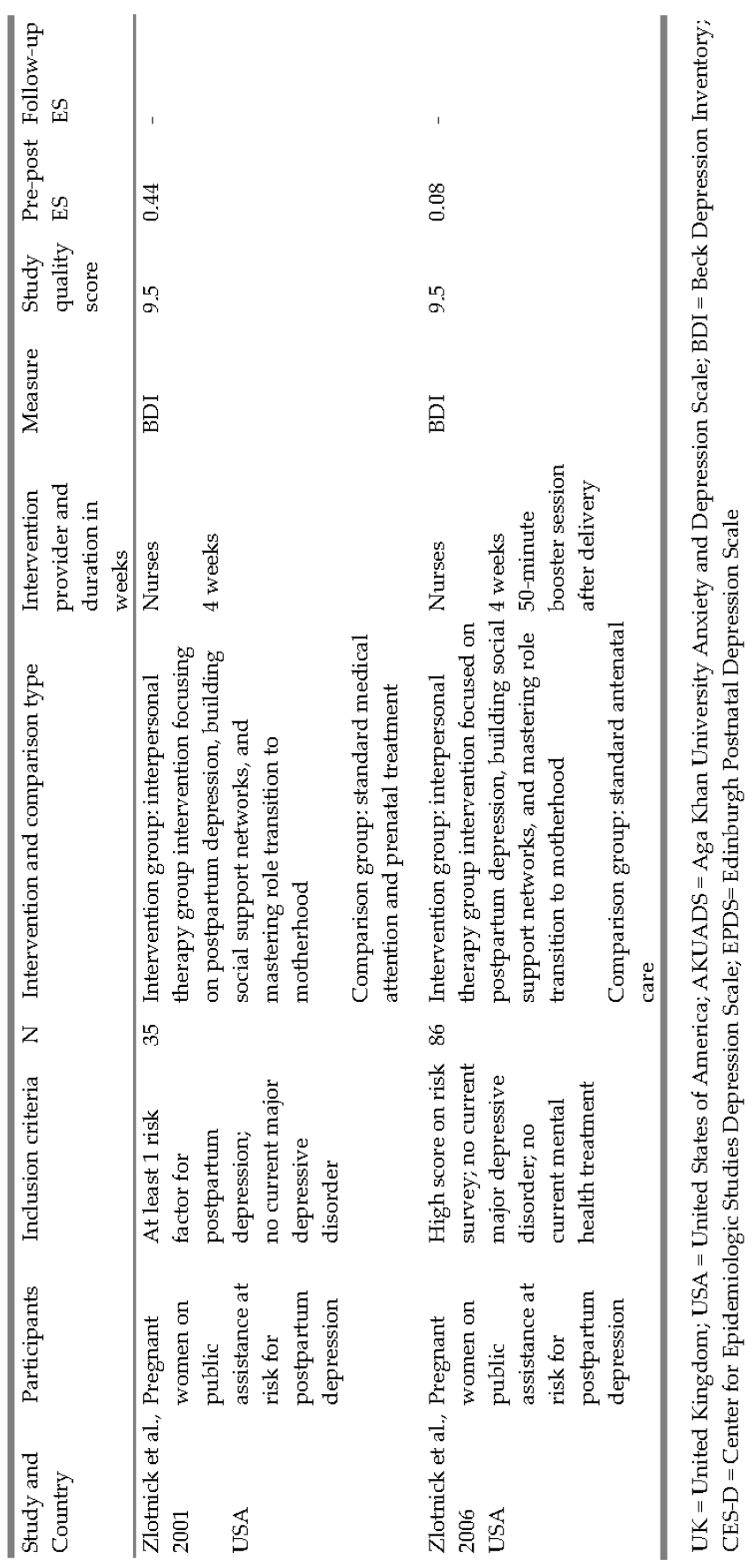


Regarding study characteristics all studies but one used a randomized controlled design. Lara et al. (2003b; 2003a) allocated intervention conditions to particular health institutions, but subjects were not subsequently randomly assigned to the different intervention conditions. Sixty-four percent of the studies used care as usual as type of comparison group, while information only was used in $29 \%$ of the studies and an alternative intervention in $7 \%$. Seven interventions (Crockett et al., 2008; Laperriere et al., 2005; Lara et al., 2003a; Muñoz et al., 2007; Tezel \& Gözüm, 2006; Zlotnick et al., 2001; Zlotnick et al., 2006) excluded the presence of major depression at baseline through the use of a clinical case-finding instrument; three studies (Ali et al., 2003; Beeber et al., 2004; Peden et al., 2005a) used depression self-reports to determine at- risk status with additional questions on the use of antidepressant drugs or therapies; and four studies (Bullock et al., 1995; Lipman \& Boyle, 2005; Wiggins et al., 2004; Zayas et al., 2004) used self-report instruments only or were targeted at high-risk women and did not assess the presence of a depressive disorder at baseline.

Study quality ranged from 8 to 13 points $(\mathrm{M}=10.57 ; S D=1.43)$. On average, therefore, the methodological quality of the selected studies was considered adequate. Eight studies (Beeber et al., 2004; Crockett et al., 2008; Laperriere et al., 2005; Lara et al., 2003a; Lipman \& Boyle, 2005; Muñoz et al., 2007; Peden et al., 2005a; Wiggins et al., 2004) presented follow-up data; the timing of followup assessments ranged from 1 to 24 months after the intervention, the commonest being 6 and 12 months. Selected characteristics of the studies are presented in Table 3.1.

\section{Overall results}

For 14 studies, sufficient data were present to calculate intervention effect size. The overall weighted mean effect size was 0.31 (95\% CI [0.17; 0.45]). Although the $\mathrm{Q}$ statistic was not significant ( $p=0.11$ ), $\mathrm{I}^{2}$ was 32.89 , indicating that some amount of heterogeneity was present. Figure 3.2 shows the Forrest plot for the overall mean effect size with $95 \%$ confidence intervals. Tezel and Gozüm's (2006) study, however, might have unduly influenced the outcomes because its effect size was outside the mean range. When removing this study, the overall mean effect size becomes $0.27(95 \%$ CI $[0.15 ; 0.38])$. 


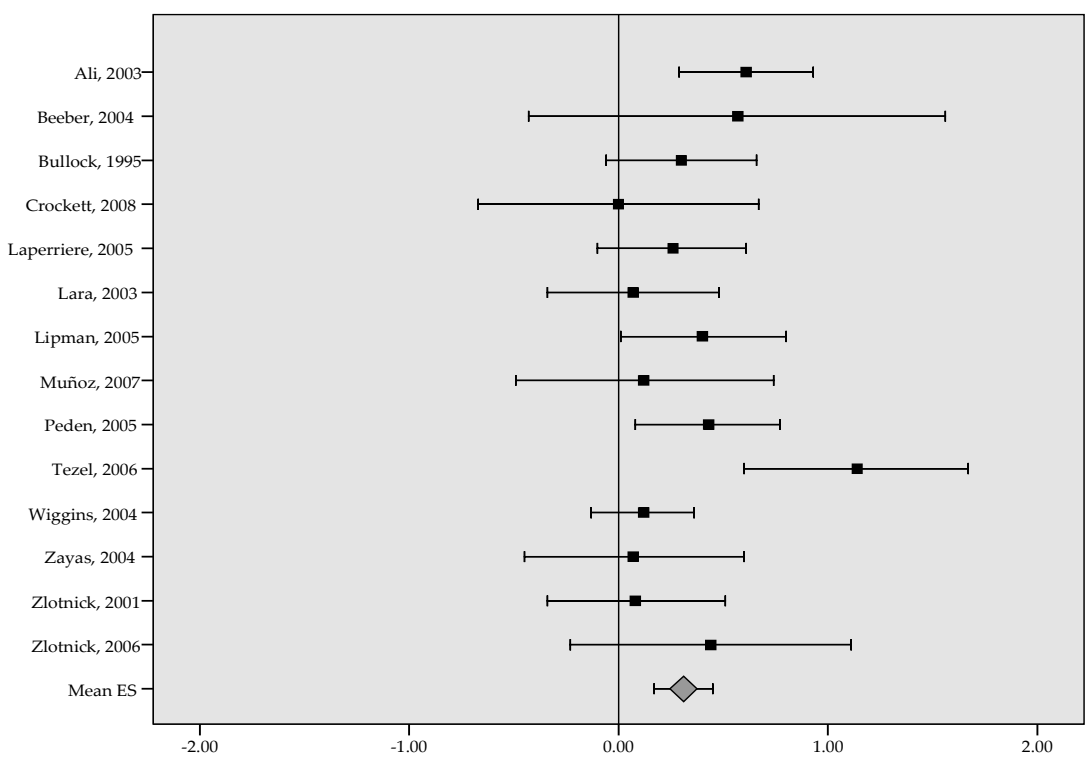

Figure 3.2 Individual and mean effect sizes $(g)$ and $95 \%$ confidence intervals of psychosocial interventions (mean effect size based on random-effects modelling using inverse variance weighting).

\section{Moderator analyses}

Moderators including sample, intervention, and study characteristics were analyzed to further explore sources of heterogeneity. The results are presented in Table 3.2.

\section{Sample characteristics}

The mean age of the study participants was not significantly related to effect size $(p=0.98)$, nor were marital status $(p=0.28)$ or perinatal versus other timing $(p=0.50)$.

\section{Intervention characteristics}

Affiliation of intervention providers was marginally related to effect size $(p<0.10)$ : studies in which nurses $(g=0.48[0.17-0.78])$, or trained nonprofessionals $(g=0.44[0.23-0.65])$ delivered the intervention had higher effect sizes than those offered by mental health professionals ( $g=0.19$ [0.05-0.34]). When controlling for the influence of Tezel and Gozüm's study (2006) this effect became nonsignificant, indicating that studies were equally effective 
when using intervention providers of different affiliations. There was no indication that intervention characteristics such as program type, program setting, delivery mode, intervention duration or booster sessions were significantly related to effect size.

Table 3.2 Univariate moderator analyses of psychosocial interventions.

\begin{tabular}{|c|c|c|c|c|c|}
\hline Variable & $\mathrm{k}$ & $\mathrm{g}$ & $95 \% \mathrm{CI}$ & Q & $p$ \\
\hline All studies & 14 & $0.31^{* * *}$ & $0.17-0.45$ & 19.37 & 0.11 \\
\hline \multicolumn{6}{|l|}{ Sample characteristics } \\
\hline Mean age & 14 & $B=-0.0003$ & $-0.03-0.03$ & 0.0005 & 0.98 \\
\hline Martial status (\% married) & 13 & $\mathrm{~B}=0.003$ & $-0.002-0.007$ & 1.28 & 0.26 \\
\hline Timing & & & & & 0.50 \\
\hline Prenatal & 7 & $0.26^{*}$ & $0.08-0.44$ & 11.13 & \\
\hline Postnatal & 7 & $0.35^{* * *}$ & $0.17-0.51$ & 4.85 & \\
\hline \multicolumn{6}{|l|}{ Intervention characteristics } \\
\hline Program type & & & & & 0.33 \\
\hline Psycho-education & 7 & $0.38^{* * *}$ & $0.22-0.54$ & $13.53^{*}$ & \\
\hline Interpersonal therapy & 4 & 0.18 & $-0.13-0.49$ & 1.61 & \\
\hline Social support & 3 & $0.23^{*}$ & $0.05-0.41$ & 1.56 & \\
\hline Mode of intervention delivery & & & & & 0.16 \\
\hline Group course & 9 & $0.23^{*}$ & $0.05-0.41$ & 3.13 & \\
\hline Individual & 5 & $0.43^{* * *}$ & $0.21-0.64$ & 8.86 & \\
\hline Program setting & & & & & 0.16 \\
\hline Community center & 9 & $0.23^{*}$ & $0.05-0.41$ & 3.13 & \\
\hline Home based & 5 & $0.43^{* * *}$ & $0.21-0.64$ & 8.86 & \\
\hline Intervention duration (weeks) & 14 & $B=-0.005$ & $-0.01-0.001$ & 2.59 & 0.11 \\
\hline Presence of booster sessions & & & & & 0.22 \\
\hline No & 10 & $0.34^{* * *}$ & $0.20-0.48$ & 13.05 & \\
\hline Yes & 4 & 0.12 & $-0.20-0.45$ & 0.87 & \\
\hline Intervention providers & & & & & 0.07 \\
\hline Mental health professional & 8 & $0.19^{*}$ & $0.05-0.34$ & 3.82 & \\
\hline Nurse & 3 & $0.48^{*}$ & $0.17-0.78$ & $8.65^{*}$ & \\
\hline Trained non-professional & 3 & $0.44^{* * *}$ & $0.23-0.65$ & 1.65 & \\
\hline \multicolumn{6}{|l|}{ Study characteristics } \\
\hline Type of comparison group & & & & & 0.01 \\
\hline Care as usual & 9 & $0.27^{* * *}$ & $0.13-0.41$ & 8.83 & \\
\hline Information only & 4 & $0.26^{*}$ & $0.08-0.45$ & 1.34 & \\
\hline Alternative intervention & 1 & $1.14^{* * *}$ & $0.59-1.69$ & 0.00 & \\
\hline MD exclusion at baseline & & & & & 0.21 \\
\hline Yes & 10 & $0.36^{* * *}$ & $0.21-0.51$ & 14.62 & \\
\hline No & 4 & $0.21^{*}$ & $0.03-0.39$ & 1.72 & \\
\hline Study quality & 14 & $B=-0.007$ & $-0.11-0.09$ & 0.02 & 0.89 \\
\hline
\end{tabular}

k=number of studies; g=Hedge's g; 95\% CI=95\% confidence interval around the effect size; $\mathrm{Q}=$ test statistic assessing heterogeneity; $\mathrm{B}=$ point estimate of the regression slope, indicating how much the effect size increased with one unit of the variable; ${ }^{*} p<0.05$; ${ }^{*}$ $p<0.01 ;{ }^{* * *} p<0.001$ 


\section{Study characteristics}

Studies using an alternative intervention as comparison group showed larger effect sizes $(g=1.14$ [0.59-1.69]), mainly due to the influence of Tezel and Gozum's study. When controlling for this study, the effect for type of comparison group became non-significant, indicating that studies were equally effective when using care as usual or information only as comparison condition. Neither study quality scores nor determination of baseline depression caseness showed significant relation to effect size.

\section{Effects over time}

In addition to post-intervention assessment, effect sizes were calculated for follow-up after 6 months $(n=5 ; E S=0.12 ; 95 \% C I[-0.05,0.27])$ and after 12 months $(n=4 ; E S=0.24 ; 95 \%$ CI [-0.04, 0.53]). Neither effect size was significant, nor were significant differences found between the overall post-intervention effect size and those at follow-up. Due to the small number of studies reporting follow-up effect sizes, it was thus deemed not meaningful to conduct moderator analyses on these data.

\section{Publication bias}

Inspection of the funnel plot for the overall weighted mean effect size showed no apparent asymmetry, supported by the results of Egger's (1997) regression intercept (bias $=0.61,95 \%$ IC $[-1,93-3,16] ; n=12 ; p=0.61$ ). Using the failsafe $\mathrm{N}$ calculation suggested by Rosenthal (1979), we calculated that 84 nonsignificant studies would need to be added to this meta-analysis to reduce the obtained mean effect size to a nonsignificant level.

\section{Discussion}

This is the first meta-analysis studying the effectiveness of psychosocial interventions on reducing depressive symptoms in low-SES women. Findings from 14 evaluated interventions indicated significant positive effects with an overall effect size of $0.31(95 \%$ CI $[0.17 ; 0.45])$. The failsafe $\mathrm{N}$ necessary to reduce this finding to a null effect would be six times the number of identified studies in the literature, all reporting negative results. This confirmed confidence in the outcomes of this meta-analysis. The weighted mean effect size of 0.31 is small to moderate (Cohen, 1988), and consistent with those found in earlier meta-analyses for depression prevention (Jané Llopis et al., 2003) and 
mental health promotion (Wilson \& Lipsey, 2001). Although the follow-up effect sizes were not significant, a trend emerged in which the significant shortterm reduction of depressive symptoms declined over time.

This meta-analysis focussed on interventions specifically designed for and targeted at low-SES adult women. Almost three quarters $(n=10)$ of the interventions in this meta-analysis focused on preventing depressive symptoms in the pre- or postpartum period, or on supporting women with children. This contributed to a fairly young overall sample; the mean participant age was 28. Depression prevention can benefit not only the mother, but her child as well (Bakermans-Kranenburg, Van IJzendoorn, \& Juffer, 2003). It is evident that the pre- and postpartum period may be an attractive preferred time to reach low-SES women through preventive interventions, as their attendance at community health clinics makes them more accessible for intervention and study recruitment. However, as there was found no difference in effects between interventions in the perinatal period and those with other timing, it is also important to address low-SES women who have passed the childbearing age.

Ethnic minority women are often over-represented among disadvantaged groups (Nadeem, Lange, \& Miranda, 2008). It is of note that most of the included studies, in particular those from the United States, were conducted with multi-ethnic participants. Of the fourteen reviewed interventions, only those by Ali et al (2003), Lara et al (2003) and Tezel and Gözüm (2006) had ethnic homogeneous participant groups. It is important to bear in mind that the studies describing their participants exclusively as low-income actually consisted for a considerable part of women from minority groups (e.g. Beeber et al , 2004; Muñoz et al, 2007) or included multiple ethnicities (e.g. Peden et al, 2005; Zlotnick et al, 2006). Thus, when we refer to low-SES women this concerns by no means a uniform group. Unfortunately, it was not possible to carry out analyses to explore possibly differential impact of the interventions in relation to ethnicity, due to too low power within each of these subgroups.

The study did not find evidence for the moderating impact of intervention characteristics that were identified in some other meta-analyses on prevention outcome studies, such as program duration, delivery mode, program type and use of a booster session (Cuijpers et al., 2008; Jané Llopis et al., 2003). This might confirm the inconclusiveness on the moderating effect of intervention characteristics that is reflected by the results of other studies. For instance Jané Llopis et al. (2003) found that preventive interventions with a duration longer then 8 sessions were more effective in reducing depressive symptoms then shorter interventions, while Horowitz and Garber (2006) restricting themselves 
to interventions for children and adolescents found no effect of intervention duration. Only the type of intervention provider reached a marginal level of significance in our study, suggesting that especially community-based nurses and trained nonprofessionals might be more successful providers for low-SES women then academically trained mental health professionals.

Explanations for these findings might lie in possible communication difficulties between generally middle-class and university educated mental health professionals and disadvantaged client groups (McLeod, Johnston, \& Griffin, 2000). These difficulties can be bidirectional in the sense that mental health providers can have difficulties addressing the central role of chronic stressors in low-SES clients' problems (Falconnier \& Elkin, 2008), while at the same time disadvantaged women often report a lack of trust in professionally trained mental health professionals (Jesse, Dolbier, \& Blanchard, 2008; Sleath et al., 2005). Thus, interpreting this finding in the present study, it might be that community-based nurses and trained nonprofessionals are more capable of conveying relevant themes and relating to the lifestyles low-SES women. Overall, it can be said that the role of non-mental health professionals as intervention providers remains indistinct, given that some previous metaanalyses reported that non-professionals alone are not the best providers, while others have disputed this view (Cuijpers et al., 2008; Den Boer et al., 2005; Jané Llopis et al., 2003). Further research should reveal the relevance of these provider characteristics in the case of low-SES target groups.

The differences in study effects may also be explained by moderators we did not consider, including attendance rates, implementation quality and fidelity, and intervention providers' supervision and training. Although the study quality of the various trials was included here as a possible moderator of evidence-based intervention effects, it was not related to the results. With a mean score of 10.5 points - given our inclusion criteria - most studies scored relatively well on overall study quality. An interesting question is whether the original studies took moderating variables into account when analyzing their data. Of the 14 studies in this meta-analysis, only 2 considered within-study moderator variables. Future prevention research should pay attention to increasing knowledge about moderators, as it informs both research and practice about the possible limits of interventions and about who benefits the most.

Of 14 studies, 8 presented follow-up data, with the timing of follow-up assessments ranging from 1 to 24 months after the intervention. A trend emerged in that the short-term reduction of depressive symptoms declined over time. This could suggest that the applied interventions were too short in 
average and had a too low dosage to generate long term effects. However, in this study length of intervention was not related to outcome. It may be that the adverse effects of low-SES women's daily circumstances are too persistent to be countered with psychosocial programs, and additional structural changes such as the alleviation of poverty, unemployment or neighbourhood disorder are required. Poverty alleviation programs and credit facilities can positively affect not just mental health, but also a range of other outcomes (Bhuiga \& Chowdhury, 2002). Due to the small number of studies reporting follow-up effect sizes, conclusions about the long-term effects of the interventions studied remain tentative.

Some limitations to this study should be noted. First, to assess the level of depressive symptom severity at pretest, four studies used depression selfreports only. In several studies, the clinical status of subjects who scored highly on depression self-report questionnaires remains unclear. Cut-off scores on such questionnaires are chosen to reflect a balance between sensitivity, specificity, and positive predictive value, though it also appears that the depressive symptomatology of high scorers does not meet the criteria of a depressive disorder as determined by a clinical interview (Santor, Zuroff, Ramsay, Cervantes, \& Palacios, 1995). This problem is also pertinent for lowSES women (Thomas \& Brantley, 2004). In the studies using self-report measures only, the total study sample can be considered at high risk for depression, of which only a small proportion might actually qualify for a depressive disorder. Indeed, depression caseness will likely be relevant to only a limited part of the total sample in the interventions included. Moderator analyses showed no significant differences in effect size between studies that excluded depressive disorder at baseline and those that did not assess the potential presence of this disorder.

This meta-analysis did not assess the outcomes of prevention on depression incidence. Only a sub-sample of the included trials determined depression incidence at posttest, rendering analysis not meaningful. Research indicates that depression may best be conceptualized as a continuum with no symptoms on one end and a major depression disorder on the other (Kessler, Zhao, Blazer, \& Swartz, 1997; Solomon, Haaga, \& Arnow, 2001). The prevention of depressive symptoms or reduction of their intensity is also relevant for preventing depression incidence (Clarke et al., 2001; Willemse et al., 2004). Furthermore, depressive symptoms can in themselves reduce quality of life and increase service use (Cuijpers et al., 2004). Thus, preventing depressive symptoms or reducing their burden can significantly benefit those receiving the intervention. 
Despite our extensive search for studies that met our selection criteria, only 14 were included in this meta-analysis. Moreover, one study (Tezel \& Gözüm, 2006) in particular seemed to have a score outside the mean range. Although the overall mean effect size was reduced from 0.31 to 0.27 after elimination of this study, on the whole it did not change the interpretation of the findings to a large extent. We may have excluded a limited number of relevant studies due to the absence of information on the percentage of low-SES women within the overall study sample. Calculation of the failsafe $\mathrm{N}$ resulted in a high number of studies that would need to be included to reduce the effects to nonsignificance, which suggests that our results are fairly robust against publication bias even if we inadvertently excluded potentially relevant studies.

Knowledge and understanding of potential moderators are essential for understanding intervention effects and improving program outcomes. Even though the focus of this meta-analysis on low-SES adult women allows the reduction of variation between the populations of the different interventions included, we have not been able to provide a clearer indication of which moderators might influence intervention effects for this target group. This might be due to the little variation in study outcomes among included studies, indicated by the low heterogeneity in this meta-analysis.

This meta-analysis showed that a number of promising programs have been developed specifically for low-SES women, a population at high risk for developing major depression. On average these programs were found to reduce the level of depressive symptoms, with more than half of the studies showing medium to large effect sizes. These findings indicate that considerable mental health benefits can be gained among disadvantaged women and can be seen as a first step toward the goal of reducing incidence of major depression in this group. Previous studies have highlighted the need for more accessible and culturally sensitive mental health services to minority and low-income communities (D'Angelo et al., 2009; Podorefsky et al., 2001). The outcomes of the present study might encourage the development of targeted psychosocial interventions specifically designed to suit this population. An opportunity for expanding the range of effective interventions lies in the development of interventions aimed at reducing stress in this group. Evidence is accumulating on the important role of stress factors in the development of depressive symptoms and major depression in disadvantaged women, possibly indicating causality (Bassuk, Buckner, Perloff, \& Bassuk, 1998; Sterk et al., 2006). To our knowledge, only a few interventions have been developed since the 1980s, when Tableman $(1987 ; 1989)$ designed and evaluated a multidimensional stress management training program for low-income women. Although the effect 
evaluations of this intervention had methodological problems, the results suggested that stress and depression could be reduced in women from lowincome populations.

Most of the interventions included in this meta-analysis were monocomponent interventions. However, to alleviate structural stressors, combinations of intervention themes and methods in multiple domains of women's lives could further increase mental health outcomes. To this end, there is increasing evidence that multi-component interventions targeting both the individual and the community could bolster outcome effectiveness (Greenberg, Domitrovich, \& Bumbarger, 2001; Jané- Llopis et al., 2003; Nation et al., 2003) Despite these advances, low-SES women in high need for assistance remain often difficult to reach. They identify more barriers to entering mental health services and the retention rate of these women in interventions is particularly low (Alvidrez \& Azocar, 1999; Senturia et al., 1998). Hence, not only the development of targeted psychosocial interventions is important, but simultaneously more knowledge should be generated on ways to overcome structural and personal barriers and the use of mental health services among this group.

During our literature search we have come across several psychosocial programs aimed at disadvantaged women, (Baxter \& Kahn, 1999; Hudson, Campbell-Grossman, Keating-Lefler, \& Cline, 2008), which have only been evaluated with quasi-experimental designs. Outcomes from rigorously performed randomized controlled trials might further expand our knowledge on effectiveness of these kinds of interventions. At the same time, much room remains for improvement in our knowledge of factors that would allow for understanding of the characteristics of successful programs. These topics have not yet been explored in detail in this group and are sorely needed. While this meta-analysis looked at factors that might moderate intervention effects, these outcomes remained inconclusive. Future studies should address this question since it will help design and implement more effective interventions. As the terms 'low-SES' and 'disadvantaged' can have differ meanings varying per nation, it is of importance that more of these studies will be conducted outside Western countries, especially in low and middle income countries. Despite these unresolved issues, this meta-analysis on effects of psychosocial interventions designed for low-SES women has shown that interventions aimed at reducing depressive symptoms are able to make small but valuable impacts in the lives of disadvantaged women. 
70 


\section{Chapter 4}

\section{Exploring recruitment, willingness to participate, and retention of low-SES women in stress and depression prevention}

Judith E.B. van der Waerden, Cees Hoefnagels, Maria W.J. Jansen and Clemens M.H. Hosman

BMC Public Health 2010;10:588-596 


\section{Abstract}

\section{Background}

Recruitment, willingness to participate, and retention in interventions are indispensable for successful prevention. This study investigated the effectiveness of different strategies for recruiting and retaining low-SES women in depression prevention, and explored which sociodemographic characteristics and risk status factors within this specific target group are associated with successful recruitment and retention.

\section{Methods}

The process of recruitment, willingness to participate, and retention was structurally mapped and explored. Differences between women who dropped out and those who adhered to the subsequent stages of the recruitment and retention process were investigated. The potential of several referral strategies was also studied, with specific attention paid to the use of GP databases.

\section{Results}

As part of the recruitment process, $12.1 \%$ of the target population completed a telephone screening. The most successful referral strategy was the use of patient databases from GPs working in disadvantaged neighborhoods. Older age and more severe complaints were particularly associated with greater willingness to participate and with retention.

\section{Conclusions}

Low-SES women can be recruited and retained in public health interventions through tailored strategies. The integration of mental health screening within primary care might help to embed preventive interventions in low-SES communities. 


\section{Introduction}

Women with low socioeconomic status (SES) and those living in disadvantaged circumstances are at high risk for not only physical health problems, but also mental health problems like depression (Adler \& Rehkopf, 2008; Kuehner, 2003). Although in recent years effective interventions have been developed for primary prevention of depression, low-SES women who are at high risk are often difficult to reach with preventive mental health services (Alegria, Bijl, Lin, Walters, \& Kessler, 2000; Gadalla, 2008). Further, they drop out of preventive interventions more frequently than their wealthier or more highly educated counterparts (Ayuso-Mateos et al., 2007), resulting in low retention rates (Beeber et al., 2007; Senturia et al., 1998). Recruitment, willingness to participate, and retention in interventions are indispensable for successfully reducing public health problems such as depression (Glasgow, Vogt, \& Boles, 1999). However, knowledge on which recruitment techniques are most effective for this population, or which characteristics of low-SES women are associated with their successful participation and retention in interventions is limited.

The most common methods for intervention recruitment are self-referral in response to announcements in local newspapers, TV and radio programs, and mass mailings (Arean, Alvidrez, Nery, Estes, \& Linkins, 2003; Gilliss et al., 2001). However, previous research has indicated that a more personalized approach might be more suitable for recruiting disadvantaged women and educating them about the availability and utility of mental health services (Alvidrez, 1999; Le, Lara, \& Perry, 2008). This approach might entail phone calls, targeted mailings, face-to-face referrals, and consultations by local community services (Azocar, Miranda, \& Dwyer, 1996; Miranda \& Green, 1999; Muñoz \& Ying, 1993). Simultaneous use of multiple methods allows mental healthcare providers to reach a diverse ethnic and socioeconomic sample (Gilliss et al., 2001), but also implies the need for a more active role in contacting potential participants. To increase disadvantaged women's willingness to participate, intervention strategies should be adapted to their needs and values (Alvidrez \& Azocar, 1999; Arean \& Miranda, 1996; Nadeem, Lange, \& Miranda, 2008). With respect to retention, several barriers and facilitators have been found to affect low-SES women's use of preventive mental health services. Instrumental barriers to retention relate to costs, transportation, and time (Beeber et al., 2007; Miranda \& Green, 1999; Weinreb, Perloff, Goldberg, Lessard, \& Hosmer, 2006), while psychological barriers are associated with attitudes and beliefs about mental health and concerns about stigma (Alvidrez \& Azocar, 1999; Miranda \& Green, 1999). Removing barriers, 
for instance through increased flexibility of services, generally increases service adherence (Alvidrez \& Azocar, 1999; Anderson et al., 2006; Azocar et al., 1996).

As in several industrialized nations, the problem of recruitment and retention of disadvantaged populations in prevention is also pertinent in the Netherlands. No more than about 4000 people are reached annually with all activities for indicated depression prevention. This constitutes approximately $1 \%$ of the 359,000 people who develop depression each year, and an even lower percentage of those at risk for depression (Meijer, Smit, Schoemaker, \& Cuijpers, 2006). Moreover, participants in preventive mental health services generally have higher education levels, suggesting an underrepresentation of people with a lower socioeconomic or migrant status (ten Have, Oldehinkel, Vollebergh, \& Ormel, 2003).

To increase participation by this group, it is necessary to improve our knowledge on factors affecting their recruitment, willingness to participate, and retention in depression prevention. This study seeks to structurally map and explore this process for a preventive depression course targeted at women from disadvantaged communities.

The first aim of this study was thus to explore the effectiveness of different strategies for recruiting low-SES women from disadvantaged communities in this preventive intervention. A second aim was to identify which sociodemographic characteristics and risk status factors within the specific target group of low-SES women affect their successful recruitment and retention. A final aim was to determine our overall success rate in reaching our target population. Findings from this study could help providers understand how to better engage low-SES women in public mental health and which women from this high-risk group are more likely to participate in interventions aimed at preventing depression.

\section{Method}

\section{Sample}

The target group for the present study was low-SES women aged 20-55 years, with elevated stress or depressive symptoms. They were recruited for participation in the Exercise without Worries (EWW) prevention course. EWW is provided by the prevention department of the district community mental health center in collaboration with the local Public Health Service. The main goal of this intervention is to reduce stress and depressive complaints and increase coping related competences by empowering the women through their strengths and resilience. In eight two-hour sessions physical exercise and 
psycho-education are combined to address evidence-based psychosocial risk factors for stress and depression in low-SES women. Topics covered in the psycho-education relate to specific problems associated with low-SES status and deal with issues such as depression, recognition of signals of tension in the body, constructive thinking and assertiveness. The exercise component focuses on stretching, muscle reinforcement, flexibility, body focused exercise and relaxation. The core element of the EWW course is its group-based format in which psycho-educative topics are combined with body-focused exercises. In each session, psycho-education and exercise components are coordinated as far as possible in an effort to reciprocally reinforce the message. The intervention features are described in more detail elsewhere (Lanen, Gelissen, Ebben, \& van der Waerden, 2008; Van der Waerden, Jansen, Hoefnagels, \& Hosman, in press).

Recruitment took place in a southern Dutch city with 36 residential neighborhoods, nine of which are considered to be disadvantaged (Theunissen \& Nijsten, 2007). The city is fairly homogeneous in ethnic terms and counts 120,175 inhabitants, 31,657 of whom are women aged 20-55 (CBS, 2008). Since years of formal education is found to be a valid single estimator of socioeconomic status (Miech \& Hauser, 2001), we used this to determine the size of the adult female low-SES population. Of the city population, $34 \%$ has completed 10 years or less of formal education, leading to an estimation of approximately 10,763 adult women as low-SES. To determine our overall success rate in reaching our target population, we used the same approach to calculate a theoretical maximum target population in the nine disadvantaged neighborhoods. The population of women aged 20-55 in these nine neighborhoods was estimated at 12,029, of whom 4289 had 10 years or less of formal education (Theunissen \& Nijsten, 2007). A recent health survey by the district Public Health Service showed that $53.5 \%$ of low-SES women had experienced moderate to severe depressive symptoms in the past week (Hajema, 2007). This would imply that 2295 (53.5\% of 4289) women living in those neighborhoods were estimated to have moderate to severe depressive symptoms.

\section{Procedures}

Figure 4.1 systematically describes the recruitment and retention process, which consists of the following steps: recruitment (referral and screening), willingness to participate (intake and course enrolment), and retention (course participation). Recruitment took place between April 2005 and November 2007, involving several referral strategies to engage low-SES women. Twenty general practitioners (GPs) working in or near the targeted socioeconomically deprived 
neighborhoods were invited to identify from their caseloads women who might be eligible for participation, and referring them to our team for a short telephone screening. Five of these GPs gave us permission to approach all adult women (20-55 years) in their patient databases. These women were sent a letter explaining the aim of the telephone screening and asking for their participation. They were then contacted by phone one week later. Other strategies focused on enhancing direct referrals by providing information about EWW to social work and debt repayment services, and to the district mental health centre and Public Health Service. Women were also referred through posters and brochures in locations frequented by low-SES women. Finally, advertisements were placed in local newspapers, neighborhood bulletins and on a local television network, and word of mouth advertising was used as well. Including these strategies lead to inevitable cross-over of information about the intervention to other parts of the city as well.

All women referred to EWW through one of these methods were screened for stress and depressive complaints in a 10-minute telephone interview conducted by trained lay interviewers. During the screening, the women provided demographic information about their age, nationality, marital status, number of children living at home, educational attainment, occupational situation, and monthly family income. They completed the 10-item version of the Perceived Stress Scale (PSS) (Cohen \& Williamson, 1987) to assess the frequency of stress-inducing situations and feelings of stress over the past month. They also completed the 10-item version of the Center for Epidemiological Studies Depression scale (CESD-SF) (Andresen, Malmgren, Carter, \& Patrick, 1994) to determine the presence of depressive symptoms in the past week.

The next recruitment phase consisted of inviting those women who fulfilled low-SES criteria and stress or depression scores above the cut-off levels to participate in the EWW course. Those who had elevated symptom scores but did not satisfy the demographic criteria were referred to regular mental healthcare. Women who met the participation criteria and were willing to participate were scheduled for an intake meeting with an intervention staff member. Women who did not keep this appointment were contacted to schedule a new meeting, with a maximum of five attempts. The intake served multiple purposes: firstly, to gather relevant background information on the women's psychological complaints, physical restraints that might hinder their participation, and need for clinical treatment; secondly, to explain the course objectives and respond to questions; and thirdly, to establish contact between the women and the intervention provider. The intakes were preferably conducted at the local women's health center to avoid any stigma associated with mental health centers. During the course, attendance was registered and 
women who missed a meeting without having notified the staff in advance were contacted about their absence. Approval for conducting this study was provided by the Medical Ethics Committee of the Academic Hospital Maastricht/Maastricht University, the Netherlands, reference number MEC 05-004.

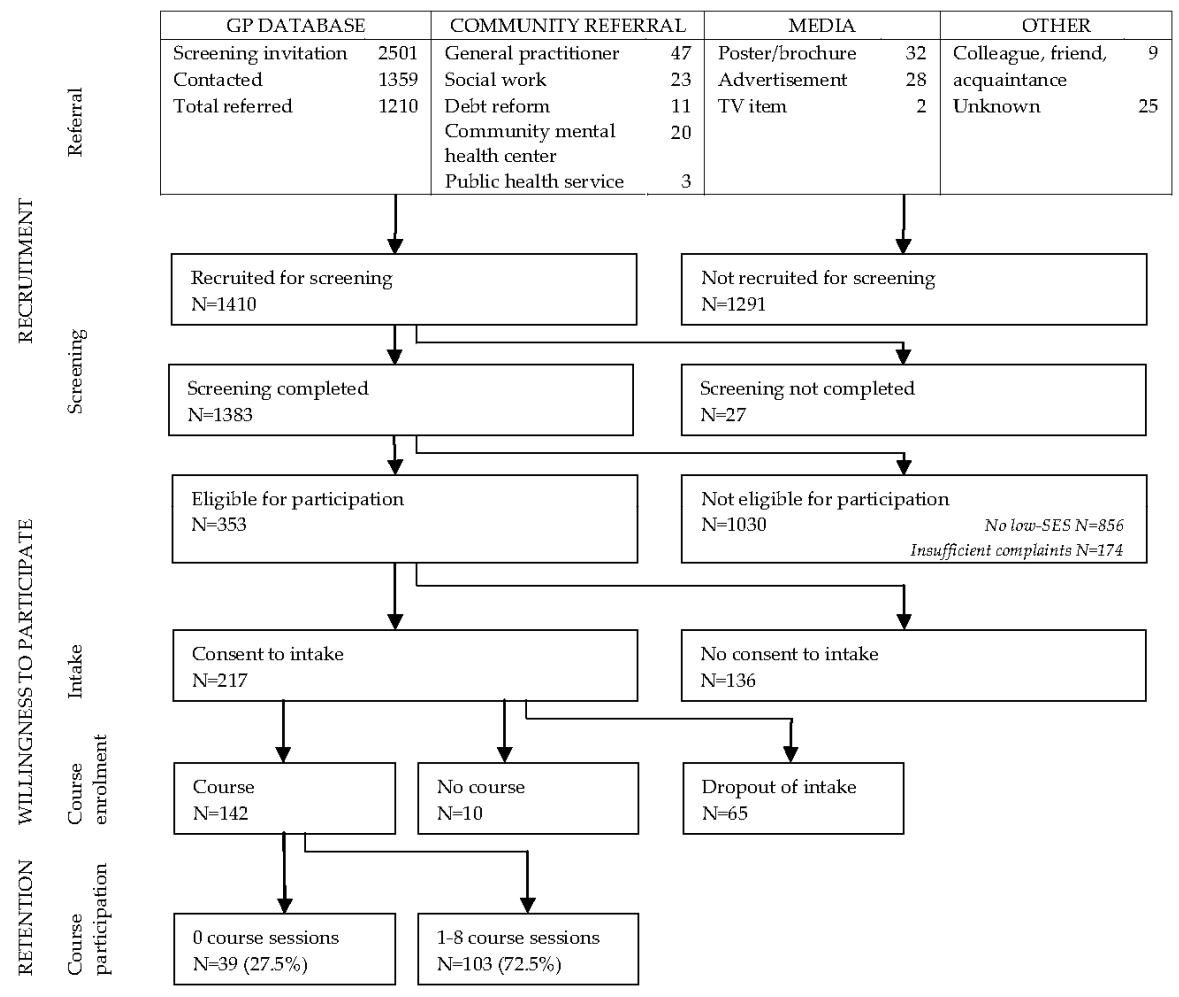

Figure 4.1 Schematic overview of the recruitment and retention process.

\section{Data analysis}

Differences in sociodemographic characteristics were compared between women who completed the telephone screening and those who dropped out during recruitment. Chi-square analyses were applied for categorical variables, t-tests, and analyses of variance for continuous variables respectively. Bonferroni corrections were applied for multiple comparisons within each separate phase. The variables were age, nationality, educational level, marital status, employment status, neighborhood prosperity score, and PSS and CESD 
scores. The type of recruitment strategy (i.e., GP database, community referral, media, or other) was tested as well, to examine whether it was related to successful completion of the screening. The same analyses were used to investigate sociodemographic characteristics and risk status factors associated with participation and successful retention in the course. To determine our overall success rate in reaching our target population, we compared a subsample of the women reached with the EWW intervention and residing in nine disadvantaged neighborhoods with the theoretical maximum target population living in those neighborhoods. We then estimated again the outcomes for recruitment, willingness to participate, and retention for our subsample, to compare with this theoretical maximum.

\section{Results}

\section{Recruitment}

A screening invitation was sent to 2501 women who were identified in the GP databases. We were able to contact 1359 of these women (54.3\%), 149 of whom refused participation. This resulted in the recruitment of 1210 women (48.4\% of those who were sent an invitation) for the screening interview through the GP databases. For the other recruitment strategies, none of the women refused to participate in the screening, resulting in 104 women recruited through community referral, 62 through the media, and 34 by other means. Of the 1410 women who were reached for the telephone screening, 1383 (98.1\%) completed the interview (see Table 4.1).

Table 4.1 Success of different recruitment strategies compared to retention rate for completion of screening

\begin{tabular}{lccccc}
\hline Recruitment strategy & $\begin{array}{c}\text { Referred for } \\
\text { screening N }\end{array}$ & $\begin{array}{c}\text { Screening } \\
\text { completed N }\end{array}$ & Eligible N & $\begin{array}{c}\text { Consent to } \\
\text { intake N }\end{array}$ & $\begin{array}{c}\text { Participation } \\
\text { in course N }\end{array}$ \\
\hline GP database & 2501 & 1194 & 256 & 124 & 76 \\
Community referral & 104 & 96 & 58 & 55 & 38 \\
Media & 62 & 60 & 22 & 21 & 14 \\
Other & 34 & 33 & 17 & 17 & 14 \\
Total & 2701 & 1383 & 353 & 217 & 142 \\
\hline
\end{tabular}

Of the total number of women screened, $856(61.8 \%)$ did not meet low-SES criteria. Of those who did, $174(12.6 \%)$ scored below the cut-off levels for stress and/or depressive symptoms. This left 353 women who were eligible for participation in the course. 


\section{Willingness to participate}

Appointments for the intake were made for 217 (61.2\%) of the eligible women. Women with complaints above the cut-off levels who did not consent to an intake had lower stress $(t(345)=-4.33, p<0.000)$ and depressive symptom $(t$ $(344)=-5.12, p<0.000)$ scores at screening than women who consented. Most women attended the first appointment. In $32.7 \%$ of the cases where the women did not attend the first appointment, two or more additional appointments had to be scheduled before the intake took place. Overall, $152(70.0 \%)$ women completed the intake. Reasons for not participating in the intake were: lost interest $(66.1 \%)$, no reason given $(18.5 \%)$, no time $(10.8 \%)$, course no longer needed $(1.5 \%)$, and medical illness (3.1\%). Those women who did not attend the intake were more often younger $(M=42.55, S D=10.26$ vs. $M=45.26, S D=9.44$; $p=.007)$ and had lower depressive symptom scores $(M=14.92, S D=7.24$ vs. $M=16.75, S D=6.58 ; p=0.006$ ). Whether or not the intake was completed was unrelated to the recruitment strategy that was used.

\section{Retention}

Of the 152 women who completed the intake, 2.0\% were excluded from participation in the course due to overly severe psychological or medical problems. A further $2.4 \%$ lost interest after receiving additional information during the intake, and $2.2 \%$ were unavailable on the days/at the times the course was offered, thus leaving 142 participants. Between September 2005 and May 2008, a total of 30 EWW groups started with an average of 9.6 participants $(S D=1.96$, range $5-13$ ) per group. Almost $30 \%$ of the eligible participants who consented to take part in the course failed to attend. For the 103 participants, attendance ranged from one to eight meetings, with a mean of $5.62(S D=2.16)$; 18 women (12.7\%) completed all eight meetings. Overall, the only characteristic associated with course completion was age. Women who completed the course were older than those who did not attend all meetings $(p<.001)$. Other demographic variables and risk status were unrelated to course attendance, as was recruitment strategy $(p>.01)$. The outcomes on stress and depressive symptoms for the participants that were successfully recruited and retained in the intervention will be tested separately in an effect evaluation.

\section{Reach of target population in low-SES neighborhoods}

The data reported above concern the total recruited sample originating from all neighborhoods. To determine our overall success rate in reaching our target population, we compared a subsample of women living in nine disadvantaged 
80 Chapter 4

neighborhoods to the theoretical maximum of 2295 low-SES women with depressive complaints living in these neighborhoods. From this selected population we were able to contact 277 women $(12.1 \%)$ for the screening interview. As Table 4.2 shows, 189 women were eligible for participation in the course. Of the 121 women who consented to an intake, 90 (74.4\%) completed the intake and 84 were willing to participate in the course. This means that $3.7 \%$ of the 2295 women were reached and that $2.7 \%$ attended the course (Figure 4.2).

Table 4.2 Reach of intended target population $(\mathrm{N}=2295)$ per recruitment phase

\begin{tabular}{|c|c|c|c|c|c|}
\hline Phases & & $\mathrm{N}$ & $\begin{array}{c}\% \text { in } \\
\text { population }\end{array}$ & $\begin{array}{c}\% \text { in recruited } \\
\text { population }\end{array}$ & $\begin{array}{c}\% \text { in eligible } \\
\text { population }\end{array}$ \\
\hline $\begin{array}{l}\text { Theoretical maximum } \\
\text { population }\end{array}$ & $\begin{array}{l}\text { Low-SES women with } \\
\text { depressive symptoms }\end{array}$ & 2295 & 100.0 & - & - \\
\hline \multirow[t]{2}{*}{ Recruitment } & Screening completed & 277 & 12.1 & 100.0 & - \\
\hline & Eligible for participation & 189 & 8.2 & 68.2 & 100.0 \\
\hline \multirow{2}{*}{$\begin{array}{l}\text { Willingness to } \\
\text { participate }\end{array}$} & Consent to intake & 121 & 5.3 & 43.7 & 64.0 \\
\hline & Participation in course & 84 & 3.7 & 30.3 & 44.4 \\
\hline Retention & $\begin{array}{l}\text { Attended at least } 1 \\
\text { session }\end{array}$ & 61 & 2.7 & 22.0 & 32.3 \\
\hline
\end{tabular}

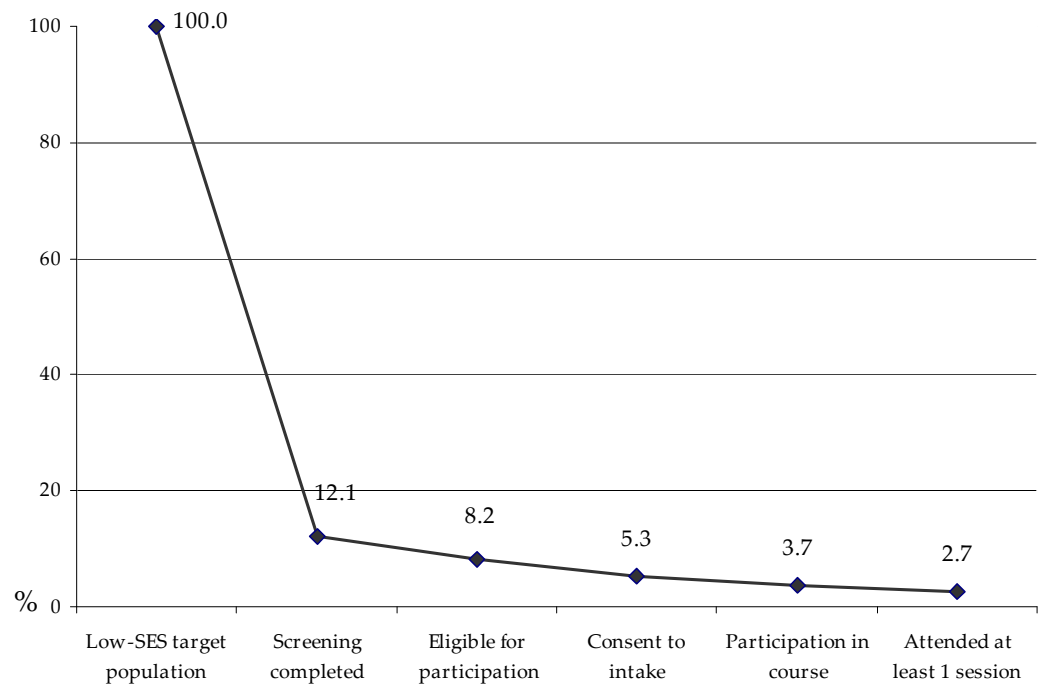

Figure 4.2 Reach of intended target population ( $\mathrm{N}=2295)$ per recruitment phase 


\section{Discussion}

Effective recruitment, willingness to participate, and retention of low-SES women in preventive interventions is critical for reducing depression in this group. To this end, we structurally mapped and explored this process for a preventive depression course targeted at women from disadvantaged communities. Based on the most conservative rate, at least $12.1 \%$ of the target population in nine specific neighborhoods completed a telephone screening as part of a targeted recruitment process. Most women were referred through the patient databases of GPs working in disadvantaged neighborhoods. Compared to strategies like community or media referral, this method resulted in more women being recruited for initial contact. Particularly older women and those with more severe complaints at screening were willing to participate and were retained in the intervention.

The effectiveness of the GP database as a referral strategy was most visible in the first stage of the recruitment process, in that it led to the largest number of initial referrals to the course. After this phase all four recruitment strategies showed equal success in terms of willingness to participate and retention in the intervention. Other effective ways to engage women were outreach through local community services and referral persons, a finding that corroborates previous research (Gilliss et al., 2001; Le et al., 2008). Further, we found that the use of carefully selected media is a suitable method to reach these women and can support the direct referral by community workers.

Thirty-nine percent of eligible women did not consent to an intake meeting even though their scores indicated an at-risk status. These women may have felt that their functioning and mental health were not heavily affected, and thus that they did not particularly need to participate in a prevention program (Ireys, DeVet, \& Chernoff, 2001). We tried to increase willingness to participate by removing instrumental barriers. Despite the fact that financial barriers are at most a minor impediment for disadvantaged populations to access mental healthcare in the Netherlands (Sareen et al., 2007), the EWW course was offered free of charge, and additional expenses for childcare and public transport were reimbursed. Also, the intervention was presented and executed as a course rather than as therapy, aiming to avoid stigmatization. Still, among those women who consented to an intake, 30.0\% did not actually attend it. One explanation might be that low-SES women are often uncomfortable saying no and will thus passively assent to appointments (Le et al., 2008), resulting in non-attendance.

Finally, increasing retention during the intervention appears to be especially important. Almost $30 \%$ of the women who consented to participate 
in the course did not attend at all, and only $12.7 \%$ completed all sessions. The low retention might influence the overall outcomes of the EWW intervention and will be considered as a potential influential factor in the effects analyses. Insufficient participation may decrease not only the intervention's effect for the women involved (Lundberg, Damström Thakker, Hällström, \& Forsell, 2005), but also the cohesion and trust within the group, and therefore potentially affect all group members. Dropouts were often younger, which corroborates the findings from previous research (Lundberg et al., 2005). To facilitate attendance, participants were called and reminded of the course shortly before its start, and those who missed a session without giving prior notification were also contacted. However, we were unable to determine whether these measures resulted in increased attendance. Other studies have shown that telephone prompting, letters, and consultations are effective means of increasing attendance rates (Azocar et al., 1996; Miranda \& Green, 1999).

In this study we were able to reach around $12 \%$ of the target population of low-SES women with a telephone screening, and $3.7 \%$ ultimately participated in the intervention. Of the people who develop depression each year in the Netherlands, only about $1 \%$ participates in preventive interventions. Moreover, these participants often belong to higher socio-economic strata, suggesting that participation might possibly be even lower for low-SES groups (Meijer et al., 2006; ten Have et al., 2003). In this study the reach was almost four times higher than the national level and possibly even higher considering that it was achieved among a low-SES population known to be hard to reach with mental health services.

One limitation of this study is that we used years of formal education as a single estimate to determine the socio-economic status of our study population. Socioeconomic status is a complex concept that is assessed using a variety of different measures. These include income, material possessions, occupational status and education, which are the concepts most commonly studied. Years of formal education has been shown to be a very good indicator of long-term economic position since it often precedes and influences employment, work, earnings, and income, thus acting as a key to positions in the stratification system (Fryers, Melzer, \& Jenkins, 2003; Ross \& Mirowsky, 2006). Furthermore, it appears that educational level is the socioeconomic indicator that is most strongly linked to mental well being and common mental disorders (Araya, Lewis, Rojas, \& Fritsch, 2003), especially so for people with few alternative resources, such as disadvantaged women (Fryers et al., 2003; Ross \& Mirowsky, 2006). Nevertheless, it is possible that by using this measure we might have excluded those women who have a high educational level, but who 
are unemployed or have low monthly incomes. Minority immigrants in particular may be disadvantaged in income attainment, as they often have access only to those sectors with lower earning potential (Nakhaie, Lin, \& Guan, 2009; Teelucksingh \& Galabuzi, 2005). However, the ethnic composition of the study location, in which $20 \%$ of the population are of non-Dutch nationality, and only $6 \%$ of non-Western origin (CBS, 2008), makes it less likely that our sample has been strongly biased in this respect.

Another limitation of this study is that contacting women for a screening via telephone means that only those with a valid number can be reached. Further, we aimed to recruit women from specific socioeconomically deprived neighborhoods, yet low-SES communities generally have high residential mobility. Thus, numerous addresses in the GP databases were not current, which may have prevented us from reaching some of our target group. Finally, we only offered one intervention. Some women may have been less willing to participate because this particular intervention did not appeal to them, or that more might have participated if we had been able to propose a multicomponent approach.

Despite the importance of successfully recruiting and retaining low-SES women in public mental health interventions, this study showed that women with stress or depressive symptoms were infrequently referred by communitybased services providers. Although successful programs for depression prevention and treatment are available (Miranda et al., 2006; Zlotnick, Miller, Pearlstein, Howard, \& Sweeney, 2006), professionals need to become more aware that disadvantaged populations are less likely to participate in such interventions. Screening women at high risk for increased depressive or stress symptoms should become a standard procedure for those providers who have frequent contact with women from disadvantaged communities. A workable solution might be 'stepped' referral, in which primary care practitioners or mental health professionals form trusted sources in the community who can identify high-risk women and subsequently refer them for screening. However, more fine-tuning is needed to identify which people within the high-risk groups need to be targeted for screening efforts. Overall, it is important that screening efforts are embedded in an appropriate care structure, so that women are not only screened for the presence of mental health problems, but also receive suitable subsequent care (Muñoz, Huynh-Nhu, \& Ghosh Ippen, 2000; Raingruber, 2002; Zayas, McKee, \& Jankowski, 2004). Particularly if a large range of preventive interventions are offered for women to choose from, their willingness to participate might increase. 
84 Chapter 4

Continued systematic investigation into recruitment and retention of lowSES groups has the potential to contribute significantly to the science of prevention. Although low-SES women are underrepresented in public health interventions, this study showed that it is possible to recruit and retain such women in a preventive intervention to a larger extent than even the middle class in the Netherlands. Integrating active recruitment and screening methods with ongoing primary care might help to embed preventive services within the settings and communities in which these women live and create win-win combinations of disadvantaged women and health care professionals. 


\section{Part II}

EVALUATION OF THE EXERCISE WITHOUT WORRIES COURSE 
${ }_{86}$ 


\section{Chapter 5}

Do disadvantaged women appreciate a synergetic exercise and psycho-educative program? Rationale and process evaluation of the Exercise without Worries course

Judith E.B. van der Waerden, Maria W.J. Jansen, Cees Hoefnagels, and Clemens M.H. Hosman

An adapted version is accepted for publication in the International Journal of Person Centered Medicine 


\section{Abstract}

\section{Background}

Stress-related complaints and depressive symptoms are highly prevalent mental health problems among low-SES women. This paper describes the theoretical background, program content, and process evaluation of the Exercise without Worries (EWW) course, a new exercise-enhanced psycho-educative intervention that targets coping with and reducing stress and depressive symptoms among disadvantaged women. This eight-week prevention course has been tailored to the specific needs of adult low-SES women.

\section{Methods}

The process evaluation used a descriptive, observational mixed methods design. Quantitative and qualitative data were gathered to determine implementation fidelity and subjective evaluations from the 135 participating women and the intervention providers.

\section{Results}

Overall attendance rates were satisfactory, but $26 \%$ of the recruited participants did not take part in the course. Implementation fidelity was sufficient, while the delivered intervention dose deviated on average for 1 out of 8 sessions from the manual. Overall, the participants reported being very satisfied with all organizational and content aspects of the intervention, with no specific preference for either exercise or psychoeducation.

\section{Conclusions}

The findings suggest that a non-stigmatizing approach of exercise and psychoeducation appears to fit with the preferences of low-SES women for preventive interventions. By extending the number of sessions and identifying reasons for nonparticipation, the program contents and execution can be improved for future adoption and implementation. 


\section{Introduction}

Women with low socioeconomic status (low-SES) are at high risk for developing mental health problems such as depression (Bassuk, Mickelson, Bissell, \& Perloff, 2002; Sterk, Theall, \& Elifson, 2006). In recent years several promising primary prevention programs have been developed, aimed at enhancing resilience and reducing vulnerability to stress and depression in this high-risk population (Beeber, Holditch-Davis, Belyea, Funk, \& Canuso, 2004; Peden, Rayens, Hall, \& Grant, 2005; Zlotnick, Miller, Pearlstein, Howard, \& Sweeney, 2006). These interventions generally apply psycho-educative, interpersonal, or social support techniques, but low-SES women may not always wish to attend these types of interventions because of perceived stigma (Alvidrez \& Azocar, 1999). However, experience from community programs suggests that exercise-based interventions might also appeal to low-SES women (Craft, Freund, Culpepper, \& Perna, 2007; Lowther, Mutrie, \& Scott, 2002). Much like other alternative intervention strategies such as herbal approaches, massage, and relaxation, exercise as a means to prevent depression is receiving increasing interest (Manber, Allen, \& Morris, 2002). Physical activity has been found to effectively reduce depressive symptoms and stress sensitivity, and may have both a preventive and a therapeutic impact on mental health problems (Craft et al., 2007; Michalsen et al., 2005; Salmon, 2001). It has been suggested that aerobic exercise according to the public health recommendations, i.e. being moderately physical active at least 150 minutes throughout the week (WHO, 2010) can effectively treat mild to moderate depression. Rates of treatment response and remission of depression with a public health dose are comparable to those reported in trials of cognitive behavioral therapy and antidepressant medication (Dunn, Trivedi, Kampert, Clark, \& Chambliss, 2005).

Since research has shown that a combination of multiple techniques is associated with increased intervention efficacy (Jané- Llopis, Hosman, Jenkins, \& Anderson, 2003), a new preventive intervention has recently been developed, implemented, and evaluated in a community setting in the Netherlands. Known as the Exercise without Worries (EWW) course, it uses a combination of physical exercise and psycho-education aimed at adult low-SES women (20-55 years) with mild to moderate depressive symptoms or stressrelated complaints. The main goal of the intervention is to reduce stress and depressive complaints and to increase the coping-related competences of disadvantaged women.

While much emphasis is placed on outcome evaluation to determine whether preventive program is effective, process evaluation may help us 
understand why a program was or was not successful (Steckler \& Linnan, 2002). Because the EWW intervention has been newly designed and implemented, it is sensible to conduct a process evaluation to see what happened in the program delivery, and how that could affect program effectiveness and efficiency (Saunders, Evans, \& Joshi, 2005). It also is of interest to determine how well the program has been accepted and evaluated by its target population. This is of special importance in low-SES groups since this population is more likely to report stigma concerns for mental health issues, which generally prohibit their use of mental health services (Grote, Zuckoff, Swartz, Bledsoe, \& Geibel, 2007). Including an exercise component in the EWW intervention might offer a solution for the low perceived attractiveness of exclusively cognitive-oriented programs among this study population. Thus, the outcomes of this study could guide future organization and implementation of a new preventive intervention that targets a generally underserviced population. The aims of this paper are twofold: first, to describe the theoretical background and program content of the EWW intervention; and second, to determine reach, adherence rates, implementation fidelity, delivered intervention dose, and participants' perception of the program by its intended audience by means of a process evaluation.

\section{Theoretical framework}

The EWW prevention course has been tailored to the specific needs of adult low-SES women by way of its focus on risk factors for stress and depression relevant for this group. The development of the intervention was guided by the stress process model of mental health disparities first proposed by Pearlin and colleagues (Pearlin, 1989, 1999; Pearlin, Menaghan, Lieberman, \& Mullan, 1981). In this model, disadvantaged social status can generate elevated levels of psychological distress through the occurrence of social stressors, while at the same time limiting access to psychological resources to manage these stressors (Aneshensel, 2009). The numerous life-event stressors and chronic problems to which low-SES women in particular are exposed include low education, low and uncertain income, unemployment, inadequate housing, single-parent status, and unrelieved child care (Ennis, Hobfoll, \& Schroder, 2000; Peden, Rayens, Hall, \& Grant, 2004; WHO, 2000). Living in poverty interferes with women's ability to exercise autonomy and decision-making latitude over stressors, and evokes a sense of humiliation, entrapment, and lack of control over life (Brown, Harris, \& Hepworth, 1995; WHO, 2000).

Stressors and chronic problems may, over time, erode the psychosocial resources that can effectively moderate the effects of stress, especially coping strategies, social support, a sense of personal control or mastery over one's life, 
and self-esteem (Bassuk et al., 2002; Roman et al., 2009; Sjögren, Kristenson, \& Linquestgroup, 2006). To varying degrees, these resources seem to be distributed by SES and are associated with mental health outcomes (Aneshensel, 2009). In response to stressors women tend to make more use of ruminative coping strategies, defined as focusing on negative mood, negative aspects of self, or stressors (Nolen-Hoeksema, Larson, \& Grayson, 1999). Ruminative coping strategies have been found to interfere with attention, concentration, and the initiation of instrumental behaviors, increasing depressed mood and contributing to a vicious cycle that maintains and deepens depressive states (Grant et al., 2004). Disadvantaged circumstances overstrain not only women's coping capacities, but also their social networks and social support (Brown \& Moran, 1997). Elliot (2001) reported greater access to social support in the form of a confidant among low-SES women, but as members of their social network are themselves likely to be poor and stressed, social networks can also serve as conduits of stress (Belle \& Doucet, 2003).

Mastery, like social support, has been conceptualized as an important resource in dealing with stress. The concept of mastery suggests both an internal locus of control as well as a positive expectancy about one's own ability to engage in behaviors needed to cope with a stressful event. Mastery may be best understood as an "executive resource" that determines the use of other resources (Green \& Rodgers, 2001). Studies have shown a negative association between low SES and belief in personal control and mastery (Taylor \& Seeman, 1999). Finally, disadvantaged women may perceive their current difficulties and past traumas to be evidence of worthlessness, and also characteristically exhibit low self-esteem (Brown, Bifulco, Veiel, \& Andrews, 1990). In sum, ongoing stress may diminish the availability of women's psychosocial protective resources to cope with stressful circumstances, thus creating both increased psychological distress and diminished resilience (Ennis et al., 2000). EWW was developed as a multi-component intervention to counter the negative effects of stressful events and increase psychosocial resources.

\section{Intervention description}

Psycho-educative programs for indicated prevention of depression, e.g., the Coping with Depression course (Brown \& Lewinsohn, 1984), usually address risk factors such as negative thought patterns, social skills, self-esteem, and pleasant activities. These topics have been extended with other evidence-based risk factors for stress and depression in the target group of low-SES women. The EWW prevention course focuses on finding a balance between burden and capacity. The core element of the EWW course is its group-based format in 
which psycho-educative topics link up with body-focused exercises. In each session, psycho-education and exercise components are coordinated as far as possible in an effort to reciprocally reinforce the message. The main focus of the intervention is on empowering the women through their strengths and resiliency, instead of focusing on their problems.

Four themes form the basis of the eight two-hour sessions, and reflect the topics covered in both the psycho-education and the exercise components (Lanen, Gelissen, Ebben, \& van der Waerden, 2008):

1. Self-image: constructive thinking, challenging negative thought patterns and attitudes about oneself and one's personal situation; increase feelings of self-respect, self-esteem, and mastery;

2. Balance: mending loss of balance between capacities and encountered difficulties; increasing number of pleasant activities, promoting resources to counter rumination, recognizing and acting upon signals of depression or tension in the body;

3. Strength: finding and reinforcing personal strengths and increasing personal control;

4. Boundaries: promoting assertiveness, indicating boundaries to reduce stressful situations.

Participants not only talk about a topic, but also experience it through the exercises. For example, during a session on strength they learn both to experience their physical strength and to discover their psychological strengths. The exercise component was added to increase the attractiveness of the program and involves stretching, muscle reinforcement, flexibility, bodyfocused exercise, and relaxation. Relaxation exercises are recommended for stress and muscle strain, especially in combination with exertion. Since lowSES women often do not have intensive exercise habits (Ball, Salmon, \& Crawford, 2006; Droomers, Schrijvers, van de Mheen, \& Mackenbach, 1998), the physical exercise was designed to be not too strenuous compared to their habitual level so as to avoid negative feelings stemming from exhaustion (Ekkekakis, Hall, \& Petruzzello, 2005; Salmon, 2001). Yet while the exercise component did not reach an intensity in conformity with public health recommendations, activity below this norm (60 to 150 min per week) has been found to have favorable effects for the prevention of depressive symptoms as well (Anderson et al., 1999; Brown, Ford, Burton, Marshall, \& Dobson, 2005). Also, beneficial effects were expected due to the psychological effects associated with engagement in exercise behavior (Brosse, Sheets, Lett, \& Blumenthal, 2002; Salmon, 2001; White, Kendrick, \& Yardley, 2009). 
Each session is administered by two trainers: a licensed psychologist or mental health provider for the psycho-education component, and an exercise professional such as a physical therapist or licensed sports instructor. Positive feedback from the trainers helps to enhance the participants' self-esteem and confidence. Their personal strengths are further reinforced by the trainers, who explicitly ask the participants to relate their own experiences and offer solutions to issues raised during the group discussions. Using participants as 'co-experts' during the sessions in this way may help increase their sense of personal efficacy (Cardemil, Kim, Pinedo, \& Miller, 2005). Social interaction and social support are encouraged within the group, including through the exercise component in which participants train with other group members (Azocar, Miranda, \& Dwyer, 1996; Brosse et al., 2002; Paluska \& Schwenk, 2000). Table 5.1 presents a comprehensive picture of the relations between the intervention components.

Barriers to participation in mental health interventions by low-SES women are often related to financial costs, transportation and time (Beeber et al., 2007; Miranda \& Green, 1999; Weinreb, Perloff, Goldberg, Lessard, \& Hosmer, 2006). Even though financial barriers form no, or at most a minor, impediment for disadvantaged populations to access mental health care in the Netherlands (Sareen et al., 2007), the EWW course is offered free of charge, and additional expenses related to child care and public transport are reimbursed. In addition, because the intervention is presented and executed as a course rather than as therapy, it is non-stigmatizing. To further this, the course takes place in community centers with which the women are familiar. The structure of the training also means that the course has a low accessibility threshold. For example, all assignments are tailored with the degree of difficulty adapted to the skills of the target group.

\section{Method}

\section{Process evaluation design}

A descriptive, observational mixed methods design was used. Qualitative and quantitative findings underpin the outcomes of this study. The analyses included the computing of descriptive statistics of participant demographics and outcomes on the evaluation questionnaires. All statistical analyses were performed using PASW Statistics version 17.0. Approval for this study was granted by the Medical Ethics Committee of the Academic Hospital Maastricht/Maastricht University, the Netherlands; reference number MEC 05004 . 
94 $\mid$ Chapter 5

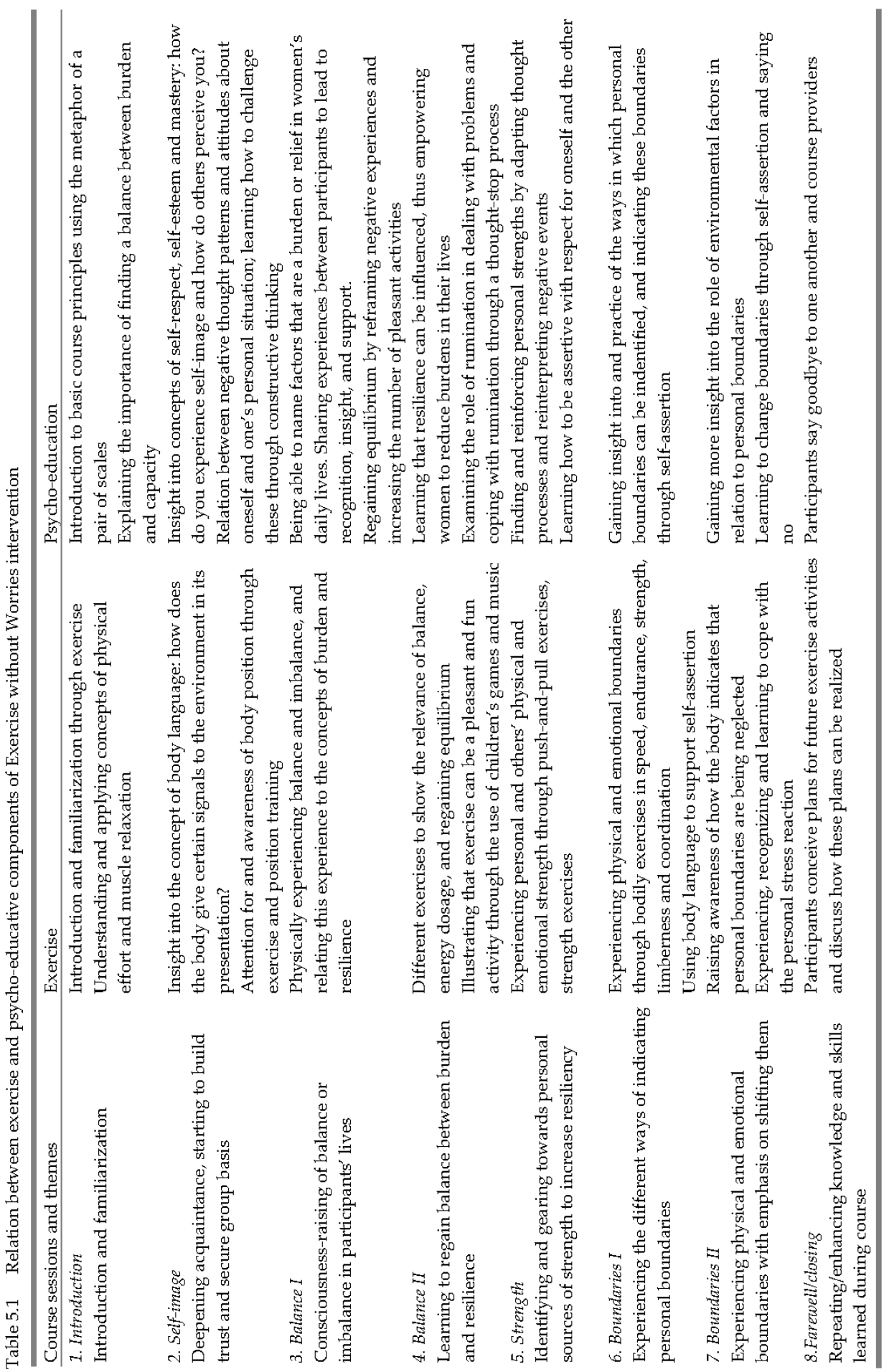




\section{Measures and data analysis}

The recommended components of a process evaluation include reach and the attendance rates of the target population, implementation fidelity, delivered intervention dose, and participant perception of program (Saunders et al., 2005).

\section{Reach}

Between April 2005 and November 2007 participants were recruited through general practitioners working in or near socioeconomically deprived neighborhoods, social workers, debt reform programs, the community mental health center and public health services, and local media campaigns. Women referred to the EWW course completed a ten-minute telephone interview conducted by trained lay interviewers to screen for the presence of stress and depressive complaints. They completed the 10-item version of the Perceived Stress Scale (PSS; range 0-40, cut-off score 14) (Cohen \& Williamson, 1987) to assess the frequency of stress-inducing situations and feelings of stress over the last month, and the 10-item version of the Center for Epidemiological Studies Depression scale (CESD-SF; range 0-30, cut-off score 5) (Andresen, Malmgren, Carter, \& Patrick, 1994) to determine the presence of depressive symptoms in the last week. Women who satisfied low-SES criteria, had stress or depression levels above the cut-off scores, and were interested in participating in the EWW course were scheduled for an intake meeting with a member of the intervention staff. During this intake, the staff member explained the objectives of the course and recorded further background information relating to the women's complaints and functional status. The intake also served to establish contact between the women and the trainer. A more detailed description of the recruitment process and willingness of low-SES women to participate in the EWW intervention is described elsewhere (van der Waerden, Hoefnagels, Jansen, \& Hosman, 2010).

\section{Attendance}

Trainers registered participant attendance for each session, and noted reasons for absence when known. To determine whether differences in attendance rates were attributable to the demographic characteristics of the participants or factors relating to the intervention itself, chi-square, and ANOVA analyses were conducted for categorical and continuous variables. To account for multiple testing, Bonferroni correction was applied. The demographic variables included were age, nationality, marital status, number of children living at home, highest educational level, and current employment status. Baseline PSS 
96 $\mid$ Chapter 5

and CESD-SF symptom scores were used as risk scores. The course-related variables were course location, day, time, group size, and trainers.

\section{Implementation fidelity and delivered intervention dose}

After each session, trainers were requested to complete a log that addressed implementation fidelity. For each session they indicated whether the course was provided as planned and as described in the course manual. By the same means, delivered intervention dose was determined. Trainers were asked to indicate for each session to what extent all of the intervention content was provided to the participants and whether there were deviations, for instance if either content was omitted or executed differently. To assess how trainers perceived the sessions, they rated the atmosphere for each session on a 6-point scale $(0=$ very poor, $1=$ poor, $2=$ average, $3=$ sufficient, $4=$ good, $5=$ excellent). Furthermore, they also had the possibility to note any particularities that occurred during a session as well as any general remarks regarding the session location.

\section{Participants' perceptions}

Participants were asked during the intake about their reasons for participating in the intervention. At the end of the last session, they completed a questionnaire to assess their experience of the program. The questionnaire was divided into questions concerning a) satisfaction with the organization of the intervention, b) appreciation of the intervention content, and c) experiences with the intervention. Satisfaction with the organization was measured with six items on a 5-point scale (1=very dissatisfied, $2=$ dissatisfied, $3=$ don't know/neutral, $4=$ satisfied, $5=$ very satisfied). These questions concerned the general content of the sessions, atmosphere during the sessions, and trainers, location, day and time.

Appreciation of the intervention content for both the psycho-educative and exercise components was assessed with a grade between 0 and 10 for each psycho-educative and exercise module, and a separate overall grade for the intervention in its entirety. Finally, participants were able to indicate in their own words what they thought the most important learning experience that they had gained through the intervention was, whether they would recommend the intervention for someone else with stress or depressive symptoms and general comments. Using these open-ended questions makes is possible to elicit study participants' feelings and explore their experiences with the course, as compared to using closed-ended questions, which limit participants' responses to a predefined set of possible answers and thus might 
introduce possible bias (Schaeffer \& Presser, 2003). The same analyses as for measuring attendance were used to investigate differences in satisfaction with intervention organization and content appreciation.

The open-ended question responses were manually coded. To this effect an organizational schema for classifying participant comments was developed. This was accomplished by reading all comments and formulating a tentative list of categories based on the comments' content and meaning. Next, comments were reread and placed in this list of categories, and if they did not fit precisely into any of these categories, either subcategories or new categories were created as needed. This process was applied to evaluate the reasons for participating in the intervention as well as for tapping in to women's experiences of the EWW course.

\section{Results}

\section{Reach}

In the period between September 2005 and May 2008, 353 women were found to be eligible for participation in the course after screening. Of the 217 women who consented to an intake, $152(70.0 \%)$ completed the intake and 135 were willing to participate in the course. They were assigned to 15 intervention groups. Characteristics of the course participants are outlined in Table 5.2. The participants' mean age was 43 years. Eighty-six percent of the women were Dutch citizens, while the rest were Moroccan (7.4\%), Turkish (2.2\%), or of other nationalities $(4.4 \%)$. Most were married or in a relationship. Forty-seven percent had completed fewer than 10 years of formal education, and $74.1 \%$ were currently unemployed. The percentage of women who had scored above cut-off at screening was $89.6 \%$ for depressive symptoms and $89.7 \%$ for perceived stress during the last month. The mean scores were 15.31 (range 029 ), and 23.27 (range 6-38) respectively, indicating that the women experienced considerable mental distress. The aforesaid percentages are below $100 \%$ and the ranges of scores on the measures include numbers below the defined cutoffs since some women had increased scores for depression, but not for perceived stress and vice versa.

\section{Attendance}

Of the 135 recruited participants, $36(26.4 \%)$ did not attend any of the course sessions. Non-participants were more often younger than others $(M=39.54$, $S D=9.09$ vs. $M=44.87, S D=8.63 ; t(126)=-3.07, p=0.003)$. None of the other 
demographic variables, baseline depression or stress symptom scores, or factors relating to the day, time, or location of the course were related to course participation. Attendance ranged between 1 and 8 meetings, with a mean of $5.84(S D=2.23)$. The most frequently cited reasons for missing sessions were health problems or work commitments. Twenty-two women $(22.3 \%)$ attended 1-4 sessions (low attendance) and 77 women (77.7\%) attended 5-8 sessions (high attendance). Differences in session attendance were not attributable to demographic variables, risk score, or course-related factors (location, time, day, group size, or trainers).

Table 5.2 Socio-demographic and clinical characteristics of study population $(n=135)$

\begin{tabular}{|c|c|c|}
\hline & $\mathrm{n}$ & $\%$ \\
\hline Age years (mean, SD) & \multicolumn{2}{|c|}{$43.41(S D=9.04)$} \\
\hline Nationality (\% Dutch) & 116 & 85.9 \\
\hline \multicolumn{3}{|l|}{ Marital status } \\
\hline Single & 34 & 25.2 \\
\hline Married/cohabitating & 87 & 64.5 \\
\hline Divorced & 6 & 4.4 \\
\hline Widowed & 8 & 5.9 \\
\hline Number of children living at home (mean, SD) & \multicolumn{2}{|c|}{$0.98(S D=1.02)$} \\
\hline \multicolumn{3}{|l|}{ Educational level } \\
\hline Low $\leq 9$ years & 63 & 46.6 \\
\hline Medium $=10$ to 14 years & 46 & 34.1 \\
\hline High $\geq 15$ years & 26 & 19.3 \\
\hline \multicolumn{3}{|l|}{ Employment status } \\
\hline Employed & 35 & 25.9 \\
\hline Housewife & 51 & 37.8 \\
\hline Unemployed & 16 & 11.9 \\
\hline Disabled & 27 & 20.0 \\
\hline Other & 6 & 4.4 \\
\hline CESD-SF score at baseline (mean, SD) & \multicolumn{2}{|c|}{$15.31(S D=6.49)$} \\
\hline PSS score at baseline (mean, SD) & \multicolumn{2}{|c|}{$23.27(S D=6.83)$} \\
\hline
\end{tabular}

\section{Implementation fidelity and delivered intervention dose}

Implementation fidelity was determined by the extent by which the course was delivered and implemented as planned in the course manual. The core element of the EWW course was its coordination of psycho-education and exercise components in each session in an effort to reciprocally reinforce the message. Of the total number of 120 course sessions (8 sessions in each of the 15 intervention groups), implementation was not delivered as planned in 22 $(18.3 \%)$ of the sessions. Deviations were: changed order of components $(5.8 \%)$; exercise component not executed (due to too few participants or absence of 
exercise trainer) $(5.8 \%)$; and psycho-education trainer absent, replaced by exercise trainer $(6.7 \%)$.

With regards to delivered intervention dose, on average 1.00 sessions $(S D=1.52)$ out of 8 deviated from the manual (range $0-5)$, either in the content of the exercise component $(M=0.64, S D=1.39)$, the psycho-education component $(M=0.83, S D=1.47)$, or both. There was no significant difference between both components in the number of deviations. For the exercise component, deviations consisted of re-adjusting the exercise to the physical capacities of the participants or omitting the relaxation exercise. For the psycho-education deviations were review of intervention content from previous lessons and additional topics discussed on request of the participants.

\section{Participants' perceptions}

\section{Reasons for participation}

During the intake the women were asked to list in their own words reasons for participation in the EWW intervention. More than one reason could be given. The most frequently cited were 'Feel better in general', which $36.4 \%$ of the women indicated, followed by 'Exercise more' (35.5\%) and 'Learn to relax' (31.4\%). Other motivations were 'Learn something about myself' (25.6\%), 'Get to know other people' (19.8\%), 'Learn to better cope with stress' (17.3\%), 'Learn to assert myself' $(14.0 \%)$, 'Learn to ruminate less' (4.1\%), and 'Feel less anxious' (1.6\%).

\section{Participant satisfaction and appreciation}

Of the 99 participants who attended at least one course session, $67 \%$ completed the evaluation questionnaire. Outcomes on a 5-point Likert scale (range 1-5) to rate satisfaction with all organizational aspects of the course, indicate that women were very satisfied with the course organization in general, with a mean score of $4.75(S D=0.38)$. The participants were least satisfied with course location $(M=4.31, S D=0.93)$, mainly due to one specific location in which the sessions were conducted in a semi-open space and thus lacked privacy. Some $(4.5 \%, n=3)$ were dissatisfied with the course hours. Participants were most satisfied with intervention staff $(M=4.93, S D=0.29)$ and session atmosphere $(M=4.93, S D=0.29)$. This corresponds with the trainers' evaluations, who rated the atmosphere during the sessions as sufficient or good for the first three sessions, and either good or excellent for the last five sessions.

Regarding satisfaction with the intervention content, the overall mean score for the EWW course was 8.71 ( $S D=0.96$, range 6.5-10.0). The scores did not relate to socio-demographic factors, symptom scores, or course factors. Grades 
for the separate exercises varied between 5.52 and 8.55 (see Table 5.3). There was no difference between the mean scores of the two course components $(p=0.83)$.

Table 5.3 Participant appreciation of the intervention topics (scale 0-10)

\begin{tabular}{|c|c|c|c|c|c|}
\hline Exercise & $M$ & $S D$ & Psycho-education & M & $S D$ \\
\hline Relaxation & 8.55 & 2.39 & Assertiveness training & 7.23 & 2.97 \\
\hline Good body position & 7.89 & 2.69 & Coping with rumination & 7.18 & 2.56 \\
\hline $\begin{array}{l}\text { Muscle training: } \\
\text { abdomen/back }\end{array}$ & 7.69 & 2.61 & Learning to say no & 7.16 & 2.82 \\
\hline Stretching & 6.85 & 2.98 & Regaining balance in life & 7.05 & 2.79 \\
\hline Ball exercise & 6.26 & 3.07 & Tackling problems & 6.94 & 2.97 \\
\hline Introduction & 6.25 & 3.09 & Pleasant activities & 6.92 & 2.99 \\
\hline Strength training & 6.15 & 3.12 & $\begin{array}{l}\text { Relation between thoughts } \\
\text { and feelings }\end{array}$ & 6.79 & 2.73 \\
\hline Rope skipping & 5.77 & 3.22 & $\begin{array}{l}\text { Identifying positive/ } \\
\text { negative thoughts }\end{array}$ & 6.59 & 3.17 \\
\hline \multirow[t]{2}{*}{ Balance } & 5.52 & 3.27 & Evaluation and farewell & 6.27 & 3.37 \\
\hline & & & Recognizing feelings & 6.23 & 3.23 \\
\hline Overall & 6.90 & 1.88 & Overall & 6.84 & 1.98 \\
\hline
\end{tabular}

$\mathrm{N}=66$

\section{Experiences with the program}

Of the total participants who completed the evaluation questionnaire, $100 \%$ would recommend the EWW course to someone with stress-related complaints, and $96.9 \%$ would do so for people with depressive symptoms. Almost $70 \%(n=45)$ of the women reported that they had fewer stress and depressive complaints after completion of the course. For a subset of the participating women, post intervention scores on depressive symptoms and perceived stress were available. Outcomes of a paired sample t-test indicate that women had lower depressive symptom scores $(M=24.23, S D=11.79$ vs. $M=21.25, S D=10.47 ; t(51)=2.07, p=0.04)$ and less perceived stress $(M=23.38$, $S D=6.73$ vs. $M=20.92, S D=6.80 ; t(51)=2.62, p=0.01$ ) after the 8 -week $E W W$ course. However, to confirm whether these preliminary outcomes in symptom change and the subjective evaluations of the course's benefits can be indeed be attributed to participation in the EWW course, an effect evaluation will be conducted separately. 
The women who completed the evaluation questionnaire indicated that they had gained a variety of experiences from participating in the course. Most often, they mentioned that they learned to assert themselves.

"I've learned to say no and to think about my own needs as well. Also I've been given tools to deal with certain situations and problems."

Other frequently cited experiences were regaining the balance between feelings and thoughts, finding rest for body and mind, taking care of oneself, and recognizing that they are not the only ones who experience certain problems.

"It feels good to know that I'm not the only one having problems, everyone has her own problems or issues, but in the end it all boils down to the same thing. You can learn how other persons deal with their stress and their approaches to solve a problem."

All in all, the women reported that they felt sorry when the course came to an end; they would have preferred more than eight sessions to continue improving their skills and profiting from the positive experience.

"This course should have more sessions, I think that then it will have even more impact. Eight meetings is too short, you're just getting to know the other participants."

\section{Discussion}

This study evaluated the implementation of the Exercise without Worries intervention by means of a process evaluation, providing information on its reach, adherence rates, implementation fidelity, delivered intervention dose and the appreciation of this new program by low-SES women. To our knowledge this is the first study to examine the acceptability of a program combining exercise and psycho-education adapted to the needs and abilities of a low-SES target group. The findings of the present study indicate that the implementation of the EWW course was largely according to plan, that the attendance rates were sufficient and that the participants appreciated both the organization and contents of this new course.

Previous research has shown that low-SES women seem especially interested in psycho-educative groups that are focused on general topics related to both physical and emotional health, such as coping with stress or living a healthy life, rather than on specific psychiatric disorders (Alvidrez \& Azocar, 1999). This was also reflected in the motivations cited by the women for their participation in the EWW intervention; the most frequent reasons were 'Feel better in general', 'Exercise more', and 'Learn to relax'. While none of the participants mentioned the motivation to feel less depressed, it could be that psychological terminology was not used because of their lower SES. However, 
the reasons mentioned by the women themselves might help us to understand which grounds for participation count most for potential participants and can be used to optimize recruitment.

In all, 135 low-SES women could be reached for participation in the intervention. Attendance of at least a minimum number of course sessions is necessary for the participants to benefit from the intervention and to sufficiently internalize outcome-related skills (Jané- Llopis et al., 2003). Among the women who involved themselves in this intervention, session attendance was medium to high, with almost $78 \%$ of the women attending most (5 to 8) sessions. This attendance rate can be considered as acceptable compared to other group preventive interventions for low-SES women (Lara, Navarro, Rubi, \& Mondragon, 2003; Muñoz et al., 2007; Peden, Rayens, \& Hall, 2005). This is even more so given the fact that participants were recruited in the community without having made specific demands for help with their symptoms. However, $26 \%$ of the recruited participants did not attend the course at all, even though all women received a reminder by telephone a day before the start of each new course. Some reported explanations were related to illness or family circumstances, but it is also possible that certain women did not participate because the intervention staff was unable to allocate each woman to her preferred day or time for the intervention. Feelings of shyness or discomfort with being in groups, or stigma-related concerns may also have played a role. Previously, Nadeem et al. (2008) found that stigma concerns, which included significant concerns about others in the community knowing about personal matters, reduced the odds of low-SES women seeking group treatment for depression. Including the exercise component to the EWW intervention is likely to reduce stigma concerns associated with mental health care; for instance, participants can refer to the intervention as "an exercise class". However, it is possible that for some women the fact that the intervention was executed in community centers was perceived as an additional barrier.

With $18.3 \%$ of the sessions deviating from the manual, implementation fidelity can be considered as sufficient. In general, deviations were most often caused by changed order of intervention components or one of the trainers being absent. It happened that the exercise professionals were also qualified mental health care providers, and could meet to compensate for the absence of their partner, thus guaranteeing continuity for the participants. However, in 7 sessions on a total of $120(5.8 \%)$, the exercise component has not been executed due to absence of the trainer. The specific aim of the EWW course was to coordinate the psycho-education and exercise components as far as possible in 
an effort to reciprocally reinforce the message. This observation indicates that in future practice, adequate monitoring is required to ensure that both intervention components are executed together. In case of a wider implementation of the course, a stand-in trainer for either of the intervention components, or schooling for each trainer to replace their partner when necessary, might be necessary solutions whose feasibility is worth investigating. With regards to delivered intervention dose, on average 1 out of 8 sessions deviated from the manual. These deviations were mostly adjustments to better adapt the intervention contents to the needs of the participants. Thus, they fitted in the overall design of providing a course with a low accessibility threshold for the target group.

Overall appreciation of the intervention organization and contents was high. Also the women indicated that they had gained a variety of positive experiences from their course participation, including increased assertiveness, emotional balance, relaxation, and self-care. At the same time, they indicated that they thought the course was too short in duration, with some of them expressing concern about whether they would be able to sustain and implement what they had learned in their daily lives after only eight sessions. Most women did perceive a reduction of stress and depressive symptoms after eight sessions. Also, a subset of the participants showed a reduction in their depressive symptom and perceived stress scores. However, it has yet to be confirmed whether these preliminary outcomes in symptom change can be indeed be attributed to participation in the EWW course. This will be evaluated in a separate randomized controlled trial.

It is very well possible that long-term retention of learned skills is difficult in face of the structural stressors and problems often present in the daily circumstances of disadvantaged women. Therefore it is important that they feel self-reliant in applying the course content in everyday life. Adapting the course design by extending the number of sessions or providing the participants with tangible recommendations how they can put the course material into practice will probably be useful adjustments. Establishing booster sessions might also help low-SES women to preserve the learned skills (Whisman, 1990).

One of the limitations of this study was that although an intake meeting was used to establish contact with disadvantaged women to encourage their participation in the course, still some of the recruited women did not actually participate in the intervention. It is known that disadvantaged women often have to manage competing demands for their time and resources (Beeber et al., 2007; Weinreb et al., 2006) which may take precedence over partaking in the EWW course. Although several possible barriers to participation have already 
been addressed and removed, our knowledge as to why women decided not to participate is limited since we did not systematically follow up on reasons for non-attendance. Secondly, the analysis of implementation fidelity and dose delivered would have gained in strength if a representative sample of the course sessions could have been directly observed and coded for deviations from the manual. Due to constrictions in funding this was not possible. However, with the employed methods we have been able to get a sound grasp of the most common problems in the course's implementation. A final limitation was the fact that evaluation questionnaires were completed during the last course session, which might have led to selection bias. The women present during this session were possibly more satisfied and motivated to participate in the intervention, which might have led to a more positive image of the intervention compared to non attending women.

The Exercise without Worries intervention was designed to address the risk factors for stress and depression in low-SES women through a resiliency-based focus. The intervention was adapted to the culture, values, and surroundings of its target population. In addition, barriers to program participation were to a large extent removed. While much emphasis is placed on outcome evaluation to determine whether a prevention program is successful, process evaluation is equally important. Process evaluation looks inside the so-called black box to see what happened in the program and how that could affect program impacts or outcomes (Harachi, Abbott, Catalano, Haggerty, \& Fleming, 1999). The outcomes of this study show that the implementation of an adapted prevention program combining exercise and psycho-education is feasible, and appeals to the needs of disadvantaged women. To our knowledge, the EWW program is the first exercise-enhanced psycho-educative intervention whose utility has been tested in this specific target group. Given the high prevalence of stressrelated complaints and depressive symptoms among low-SES women, prevention is an important means of providing mental health care to this group. However, still few opportunities exist for low-income women to share their experiences, have their emotional and life experiences validated, and engage in some problem-solving and skill development (Cardemil et al., 2005). The positive experiences with the Exercise without Worries course indicate that multi-component programs combining mental and physical health are one way to better address the mental health needs of low-SES women. If the intervention appears to be effective, further dissemination of the EWW intervention can be appropriately pursued. 


\title{
Chapter 6
}

\begin{abstract}
A randomized controlled trial of combined exercise and psycho-education for low-SES women: Short- and long-term outcomes in the reduction of stress and depressive symptoms
\end{abstract}

Judith E.B. van der Waerden, Cees Hoefnagels, Clemens M.H. Hosman, Maria W.J. Jansen, and Pierre M. Souren

Submitted for publication 


\section{Abstract}

\section{Background}

The Exercise without Worries intervention aims to reduce stress and depressive symptoms in low-SES women by means of an eight-week group based program combining physical exercise and psycho-education. The effects of an exercise regimen as adjunctive to a psychosocial intervention component have not yet been tested within this population.

\section{Methods}

A total of 161 low-SES women with elevated stress or depressive symptom levels were randomly assigned to the combined exercise/psycho-education intervention $(E P, n=57)$, exercise only $(E, n=51$ ) or a waiting list control condition (WLC, $n=53$ ). Depressive symptoms (CES-D) and perceived stress (PSS) were measured before and immediately after the intervention and at 2, 6 and 12 month FU.

\section{Results}

Multilevel linear mixed-effects models revealed no differential patterns in reduction of CES-D or PSS scores between the EP, E and WLC groups on the short (2 months) or long term (6 and 12 months). Outcome levels of depressive symptoms were moderated by initial depressive symptom scores: Women from the EP and E groups with elevated, but low initial symptoms benefited from participation on the short term. Further, women in the EP and E groups with the lowest educational level reported more stress reduction at post-test than women with higher educational levels.

\section{Conclusion}

In the overall target population of women from low-SES neighborhoods, no indications were found that the Exercise without Worries course reduced depressive symptom and stress levels on the short or long term. The findings do suggest, however, that exercise alone or a combination of exercise and psycho-education may be a viable prevention option for certain groups of disadvantaged women. Especially those low-SES women with less severe initial problems or those with low educational attainment should be targeted for future depression prevention practice. 


\section{Introduction}

Compared to women with higher socio-economic status (SES), low-SES women have a high risk (ORs between 2.7 and 5.4) for depressive symptoms, which in turn considerably increases their likelihood of developing major depression (Cuijpers \& Smit, 2004; Judd, Schettler, \& Akiskal, 2002; Kahn, Wise, Kennedy, \& Kawachi, 2000). Chronic stress and daily hassles are considered strong predictors of depressive symptoms and episodes in this group (Falconnier \& Elkin, 2008; Hammen, 2003; McConagle \& Kessler, 1990). Interventions specifically developed for and aimed at disadvantaged women have been found effective in the primary prevention of depression and reduction of stress (e.g. Muñoz et al., 2007; Peden, Rayens, \& Hall, 2005; Zlotnick, Miller, Pearlstein, Howard, \& Sweeney, 2006). The most commonly used intervention types for this population are psycho-education, which includes the application of cognitive behavioral mechanisms, stress-management techniques or counseling activities, interpersonal therapy and the strengthening of social support (Van der Waerden, Hoefnagels, \& Hosman, 2010).

Exercise may have both a preventive and a therapeutic impact on mental health problems and stress, and has been found to be effective in reducing depressive symptoms (Alderman, Rogers, Johnson, \& Landers, 2003; Stathopoulou, Powers, Berry, Smits, \& Otto, 2006). Meta-analyses on the effect of exercise on depressive symptoms report effect sizes ranging between 0.35 and 0.59 (Conn, 2010). However, the optimal dose in terms of frequency, duration and intensity needed for treatment and prevention efficacy has not yet been fully defined (Atlantis, Chow, Kirby, \& Singh, 2004). There are some indications the recommended public health dose (PHD) of exercise results in remission rates comparable to those reported in trials of cognitive behavioral therapy (CBT) and antidepressant medication (Dunn, Trivedi, Kampert, Clark, \& Chambliss, 2005). In addition to its application as a monotherapy, exercise is increasingly accepted as an adjunct intervention in the management of depressive symptoms and depression (Merom et al., 2008). The underlying assumption in offering these multidisciplinary interventions is that combining different types of effective interventions will increase and prolong their positive effects (Jané- Llopis, Hosman, Jenkins, \& Anderson, 2003; Tsang, Chan, \& Cheung, 2008). However, research on the combination of exercise with empirically supported psychosocial approaches for depression has been limited (Stathopoulou et al., 2006). To our knowledge, only two randomized controlled trials have tested the preventive effects of combined CBT and exercise on the level of depressive symptoms (Fremont \& Craighead, 1987;

McGale, McArdle, \& Gaffney, 2010). The first study compared the effects of a 
combined intervention to CBT or exercise alone (Fremont \& Craighead, 1987) and the second compared a combined intervention to an exercise only group and a control group (McGale et al., 2010). While both studies did show a significant decrease in depressive symptom scores in the experimental conditions, no significant additive effects were found for a combined intervention relative to a CBT-only comparison condition.

The effects of an exercise regimen in combination with a psychosocial intervention component have not yet been tested within a low-SES population. Although a study by Craft, Freund, Culpepper, and Perna (2007) indicated that it is possible to reduce depressive symptoms in disadvantaged women by means of exercise strategies, in general they are still an underrepresented population within research on the effects of physical exercise on mental health (Albright et al., 2005). To address this evidence gap, the Exercise without Worries (EWW) course was developed and evaluated in the Netherlands combining an exercise regimen with a CBT-approach. As indicated earlier, the increased and sustained exposure to stressors among low-SES women can have an important mediating impact on their depressive symptom levels. Therefore, the EWW intervention not only aimed to reduce depressive symptoms but also the perceived stress levels of its participants.

The objective of the current study was to evaluate the effects of this preventive program aimed at reducing stress and depressive symptoms among low-SES women. We directly looked at whether the combination of exercise and psycho-education led to synergetic effects on participants' mental wellbeing in comparison to participants receiving exercise only and to a waiting list condition. Since previous research has shown differential intervention effects relating to socio-economic status (Cuijpers, Van Straten, Warmerdam, \& Smits, 2008), baseline symptom levels (Allart-van Dam, Hosman, Hoogduin, \& Schaap, 2003) and intervention adherence (Stuart, Perry, Le, \& Ialongo, 2008), we further explored whether the intervention itself would lead to differential effects among the groups based on these moderators.

\section{Method}

\section{Study design and participants}

The efficacy of the EWW course was tested in a randomized control trial. The target population of the EWW intervention were low-SES women (20-55 years) with depressive symptoms or stress-related complaints. Between April 2005 and November 2007, participants were recruited through general practitioners active in or nearby socio-economically deprived neighborhoods, social work, 
debt reform, the community mental health centers and public health services and local media campaigns. Women who were referred to the EWW course completed a 10-minute telephone interview conducted by trained lay interviewers to screen them for the presence of stress and depressive complaints. Women who satisfied low-SES criteria, had stress or depression levels above the cut-off scores (see measures), and were interested in participating in the EWW were invited to an intake during which course objectives were explained and further background information related to the women's complaints and functional status was collected. Women were excluded from participation in the course if their Dutch was limited or if they had cognitive disturbances or emotional instability that might impede participation in a group intervention or physical problems that might hinder their participation in the exercise component of the course. Those women suitable and willing to participate in the RCT signed a written informed consent form for participation and were subsequently randomly assigned using a randomization list.

Participants were assigned to one of three intervention conditions: the full EWW intervention with exercise and psycho-education (EP) component, the intervention with the exercise only (E) component or a waiting list control (WLC) condition with postponed intervention four months later. The study design did not include a psycho-education only $(\mathrm{P})$ condition, since this component has already been shown to be evidence-based in the reduction of depressive symptoms (e.g. Cuijpers, Munoz, Clarke, \& Lewinsohn, 2009; Cuijpers, Smit, \& Van Straten, 2007). Until the first session, participants were blinded to the treatment group to which they were assigned. Measurements in the EP and E conditions were performed at baseline, a post-test directly after the 8-week intervention, and 2 months, 6 months and 12 months follow-up (FU). Participants in the WLC group completed baseline measurements, a posttest and a 2 month FU at the same time as the EP and E groups. After this measurement, they were given the opportunity to participate in the intervention. During the study period, 161 eligible women gave informed consent and were randomly allocated to the EP, E and WLC conditions (Figure 6.1). Approval for conducting this study was granted by the Medical Review Ethics Committee of the Academic Hospital Maastricht/Maastricht University in the Netherlands. 


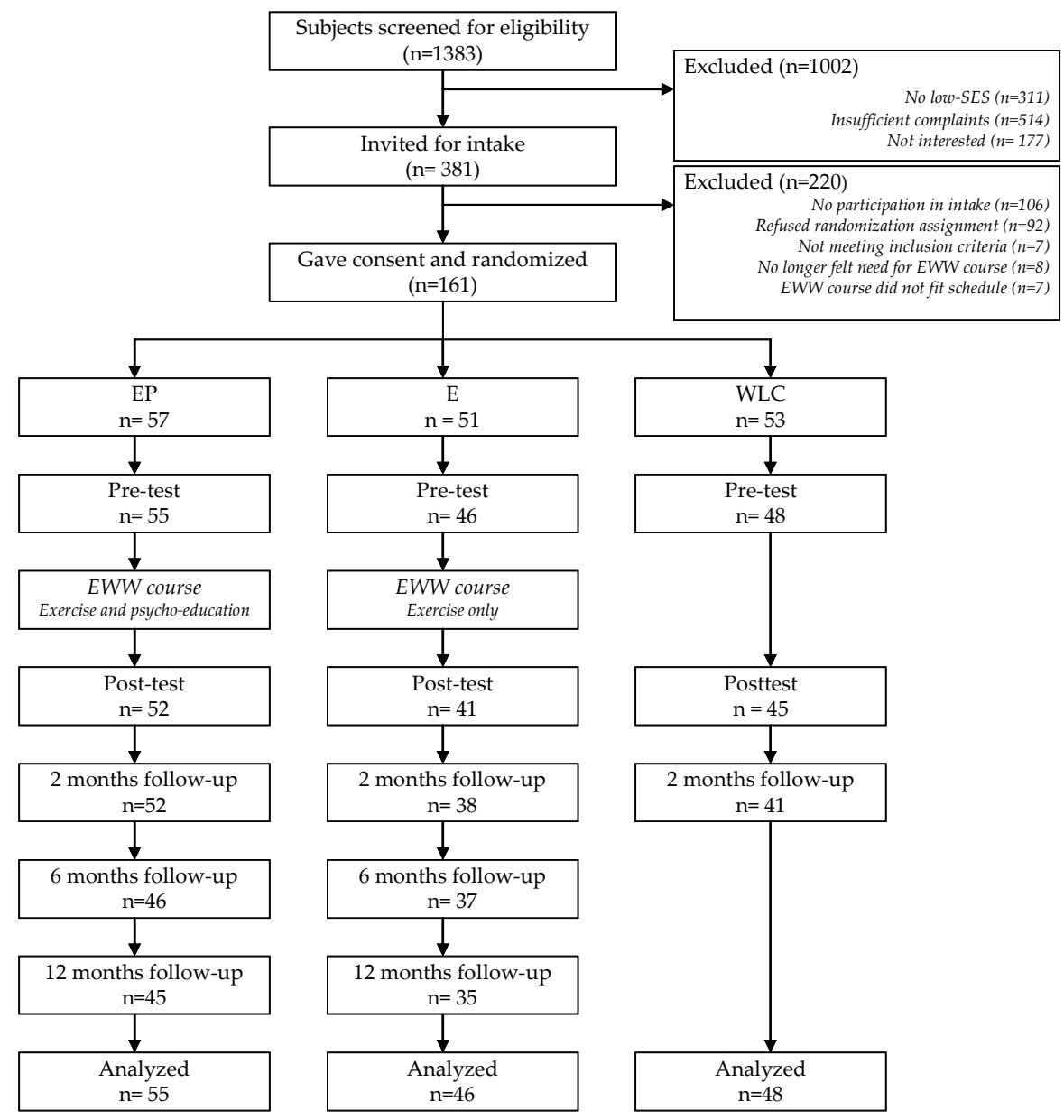

$E P=$ exercise and psycho-education; $E=$ exercise only; WLC=waiting-list control

Figure 6.1 Flow of the participants through the study.

\section{Intervention and comparison group activities}

Exercise without Worries (EWW) is a new preventive course that has been tailored to the specific needs of low-SES women. The main goal of the intervention is to reduce stress and depressive complaints and increase coping related competences. The emphasis lies on empowering women instead of focusing on their problems. One of the most important elements within the course is that each of the eight two-hour sessions links the psycho-educative subjects with the body focused exercises. The psycho-education and exercise 
components are coordinated as far as possible with the aims of reciprocally reinforcing the message. Psycho-education addresses risk factors for depression such as negative thinking, and a lack of social skills, self-esteem, and pleasant activities. These topics are extended with other evidence-based risk factors for stress and depression found among low-SES women, such as low perceived mastery over life, and the negative effects of stressful events (Ennis, Hobfoll, \& Schroder, 2000; Hauenstein, 1996). The exercise component employs stretching, muscle reinforcement, flexibility, body focused exercise and relaxation. Relaxation exercises are recommended for women with stress and muscle strain, especially in combination with exertion. Since low-SES women do not commonly have intense exercise habits (Ball, Salmon, \& Crawford, 2006; Droomers, Schrijvers, Van de Mheen, \& Mackenbach, 1998), the physical exercise was designed not to be too much more strenuous than their habitual level of exercise to avoid negative feelings combined with exhaustion (Salmon, 2001). Social interaction and social support from other participants were encouraged through the group based format, and also through the exercise component in which participants exercise together (Azocar, Miranda, \& Dwyer, 1996; Brosse, Sheets, Lett, \& Blumenthal, 2002; Paluska \& Schwenk, 2000). Each session was led by two trainers, a licensed psychologist or mental health provider and an exercise professional such as a physical therapist or licensed sports instructor. The trainers provide positive feedback to enhance participants' self-esteem and confidence. A more comprehensive description of the EWW course has been reported elsewhere (Lanen, Gelissen, Ebben, \& Van der Waerden, 2008).

The exercise only (E) condition included the same exercises as the combined program, but without the psycho-educative component. During the exercise only sessions, the refreshment breaks were extended to correspond with the time provided for the staff-client contact in the EP course. Finally, the women in the waiting list control (WLC) condition did not start the intervention until four months later and were instructed to continue life as normal and were free to engage in both physical activity and mental health counseling elsewhere.

\section{Measures}

To determine study eligibility, women completed the 10-item version of the Perceived Stress Scale (PSS; range 0-40, cut-off score 14) (Cohen \& Williamson, 1988) to assess the frequency of stress-inducing situations and feelings of stress over the last month. Also, they completed the 10-item version of the Center for Epidemiological Studies Depression (CESD-SF; range 0-30, cut-off score 5) (Andresen, Malmgren, Carter, \& Patrick, 1994) to determine whether they had experienced depressive symptoms over the last week. The cut-off scores were 
not applied to establish the presence or absence of clinical levels of stress or depression, but rather were used to select women considered to be at high risk.

All but one questionnaire were administered at participants' homes by trained interviewers to assure maximum response from the target group (Picavet, 2001). The 2 month FU measure was administered by phone. The primary outcomes in the comparison between intervention conditions were related to depressive symptoms and perceived stress. Depressive symptoms were assessed using the Center for Epidemiological Studies Depression (CES-D) questionnaire (Radloff, 1977). This 20-item questionnaire measures the number of depressive symptoms over the past week, including depressed mood, loss of interest or pleasure in activities, sleep and appetite disturbances and social difficulties. Scores range between 0 and 60 . Scores ranging from 16 to 26 are considered indicative of mild depression, and scores of 27 or more indicate a high risk of major depression (Thomas, Jones, Scarinci, Mehan, \& Brantley, 2001; Zich, Attkisson, \& Greenfield, 1990). The reliability of the CES-D scale was acceptable to good with Cronbach's $\alpha$ ranging between 0.77 and 0.91 across the various time points. These $\alpha$ are consistent with ranges found in Dutch community samples (Bouma, Ranchor, Sanderman, \& Van Sonderen, 1995).

Participants' general perceived stress over the past month was assessed using Cohen's Perceived Stress Scale (PSS) (Cohen, Kamarck, \& Mermelstein, 1983). The scale assesses the frequency of stress-inducing situations and feelings of stress over the last month, and was designed for use in the general community to determine how much stress people experience in their everyday lives. Scores range from 0 to 40 with higher scores indicating higher perceived stress levels. As the PSS is not a diagnostic instrument, clinical cut-offs for the general population have not been well established or reported on much. However, the PSS has demonstrated adequate reliability and validity (Cohen \& Janicki-Deverts, 2010; Cohen et al., 1983), and Cronbach's $\alpha$ in the present study ranged from 0.77 to 0.90 across the consecutive measurements, indicating adequate-to-good internal consistency of this instrument.

The following socio-demographic variables were determined at baseline: age, nationality, educational level, and previous depressive episodes. Employment status, net monthly family income, marital status, current depression treatment and current exercise behaviors were also established at baseline and during each subsequent time point. At the 12 month FU, participants also completed the Dutch Recent Life Events Questionnaire (RLEQ), which counts the total number of major life events experienced over the past 12 months (Willige, Schreurs, Tellingen, \& Zwart, 1985). The Cronbach's $\alpha$ in this sample was 0.89 . 


\section{Data analysis}

An a priori power analysis conducted to compare the three conditions on the primary outcome for depressive symptoms estimated a sample size of 49 participants in each condition to detect a moderate effect size $(d=0.40)$ with a power of 0.80 and a two-tailed test of 0.05 . Accounting for an estimated dropout of $10 \%, 54$ participants were needed in each condition.

The data analysis included descriptive statistics of the socio-demographic and other background variables and primary outcome measures. Women were included in the analyses if they had completed the baseline assessment. Dropout analyses were conducted using logistic regression analyses to explore differences in socio-demographic characteristics and symptom scores for those participants who did not complete all measurements after the baseline assessment. To check for preexisting differences among the three conditions on descriptive variables and outcome measures at study entry despite randomization, an analysis of variance (ANOVA) was carried out for continuous variables and Chi-square analyses were performed for categorical variables. Analyses were performed using SPSS for Windows (version 15.0).

Since participants were nested within EWW course groups and participated in multiple measurements, multilevel linear regression analyses were used to account for dependency in the consecutive outcomes (Snijders \& Bosker, 1999). A multilevel approach with random intercept for three levels (course group, participant and time) was completed to analyze the changes in outcome variables between intervention conditions and moderation effects using MLWin (version 2.02). Separate analyses were performed for each of the outcome measures. Multilevel analyses have also been shown to be useful in handing missing data, since analyses can be done on available cases instead of complete cases only (Twisk, 2006). For respondents with no more than $20 \%$ of missing values on a given scale and time point, the missing values were substituted by the respondent's mean score on that particular scale (Moons, Donders, Stijnen, \& Harrell, 2006). There were no scales with more than $20 \%$ of missing values; the maximum percentage of missing values for a given scale was for the RLEQ, with 13\% missing.

To test the change in outcome on the CES-D and PSS between the intervention conditions, a fixed occasions model was fitted for each outcome variable, with a random group effect and an unspecified $5 * 5$ covariance matrix for participant effects at all five time points. Included predictors were intervention condition variables coded into two dummies (using the WLC condition as a reference), time of measurement which was recoded into four dummies (with baseline as reference time point), and eight two-way interaction terms for each combination of intervention condition*time to test whether the 
intervention effect varied over time. Baseline socio-demographic variables, baseline PSS and CES-D scores, intervention adherence, current depression treatment and number of life events were included as between-subject covariates because these variables were assumed to be related to intervention outcomes. By including these covariates, the power and precision of the testing and estimation of the treatment effect are improved due to reduced residual outcome variance.

The overall intervention effects, and those at post-test, short ( 2 months FU) and long term (at 6 and 12 months FU) were analyzed by following a 'topdown' procedure, starting with the most elaborate model and successively leaving out the least significant components (using $\alpha=0.10$ to prevent type II errors). In the most reduced model, which contained time and group by time effects plus significant covariates, the intervention effects were statistically tested using the likelihood ratio test with a 0.05 significance cut-off point for regression coefficients. In all of the analyses, a correction was made for baseline CES-D and PSS scores, as well as for number of life events.

Differential effects of the intervention could be present in participant subgroups. We included moderators for SES indicators (educational level, employment status, and net monthly family income), intervention adherence, and risk at baseline levels (based on the sample median of the pre-test scores for the CES-D and PSS respectively). Higher order interactions of these covariates with intervention condition*time were included into the model, as well as all lower order terms involving the same covariate. If a higher order interaction was found between a covariate and group by time, the treatment effect was evaluated within subgroups based on that covariate.

The intervention effect sizes were determined using Cohen's method (Cohen, 1988) by dividing the difference between two means by the pooled SDs for those means. Effect sizes were calculated for the effect of the EP and E groups compared to the WLC group and between EP and E groups on both the CES-D and PSS. Effect sizes were interpreted as small when they were equal to or less than 0.32 , medium when they were between 0.33 and 0.55 , and large when they were equal to or greater than 0.56 (Lipsey \& Wilson, 2001).

\section{Results}

Of the 161 women enrolled in the study, 12 dropped out before the baseline assessment took place and were excluded from any further analyses. The participants who dropped out after the baseline measurement did not differ from those who completed all assessments $(p>0.05)$. Table 6.1 presents the 
socio-demographic characteristics, as well as the presence of previous depressive episodes, current depression treatment and current exercise behavior at baseline for each of the three study conditions. No significant differences were found between the three conditions for any of these variables, with the exception of nationality. All participants in the WLC group were Dutch, versus $91 \%$ in the EP condition and $84 \%$ in the E- condition $\left(\chi^{2}(2)=\right.$ 7.47, $p=0.02$ ). Furthermore, the 12 month FU showed that women in the $\mathrm{E}$ condition had experienced significantly more life events during the study period than women in the other conditions $(F(2,146)=2.97, p<0.05)$. Women attended on average $5.06(\mathrm{SD}=2.79)$ meetings, without any significant differences between the two intervention conditions.

\section{Intervention effects}

Table 6.2 shows the descriptive study measures from baseline to one-year FU. Multilevel linear mixed-effects analyses revealed that women in the EP and E, but not the WLC, conditions showed significant $(p<0.05)$ improvements on their score for depressive symptoms at post-test. For the $\mathrm{E}$ condition, the change to the pre-intervention scores was still significant at 6 and 12 month FU $(p<0.01)$. However, this change in depressive symptom scores was not significantly different $(p=0.12)$ between the EP, E and WLC conditions on any of the measurements (Table 6.3).

Outcomes on perceived stress levels demonstrated a comparable pattern. Compared to their pre-intervention scores, women in both the EP and E conditions showed a significant decrease in their perceived stress levels at posttest and FU $(p<0.01)$; there was no decrease, however, in the scores of the women in the WLC condition. Yet, overall intervention effects on decreases in stress levels between the three conditions were not significant on any of the measurement $(p=0.34)$. 
Table 6.1 Baseline characteristics of 149 participants, means (SD), frequencies (\%).

\begin{tabular}{|c|c|c|c|c|}
\hline & $\begin{array}{l}\text { EP group } \\
(\mathrm{n}=55)\end{array}$ & $\begin{array}{l}\text { E group } \\
(\mathrm{n}=46)\end{array}$ & $\begin{array}{l}\text { WLC group } \\
\quad(\mathrm{n}=48)\end{array}$ & Total sample \\
\hline Age (years) & $44.68(S D=8.43)$ & $43.06(S D=8.88)$ & $43.87(S D=7.67)$ & $43.90(S D=8.31)$ \\
\hline Nationality (Dutch)* & $50(90.9)$ & $39(84.8)$ & $48(100.0)$ & 137 ( 91.9) \\
\hline \multicolumn{5}{|l|}{ Marital status } \\
\hline Single, Divorced, Widowed & $22(40.0)$ & $26(56.5)$ & $17(35.4)$ & $65(43.6)$ \\
\hline Married, cohabitating & $33(60.0)$ & $20(43.5)$ & $31(64.6)$ & $84(56.4)$ \\
\hline \multicolumn{5}{|l|}{ Educational level a } \\
\hline Low & $27(49.1)$ & $28(60.9)$ & $24(50.0)$ & $79(53.0)$ \\
\hline Middle & $21(38.2)$ & $14(30.4)$ & $22(45.8$ & $57(38.3)$ \\
\hline High & $7(12.7)$ & $4(8.7)$ & $2(4.2)$ & $13(8.7)$ \\
\hline \multicolumn{5}{|l|}{ Employment status } \\
\hline Employed & $13(23.6)$ & $13(28.3)$ & $16(33.3)$ & $42(28.2)$ \\
\hline Housewife & $14(25.5)$ & $8(17.4)$ & $10(20.8)$ & $32(21.5)$ \\
\hline Unemployed & $12(21.8)$ & $8(17.4)$ & $4(8.3)$ & $24(16.1)$ \\
\hline Disabled & $16(29.1)$ & $17(37.0)$ & $18(37.5)$ & $51(34.2)$ \\
\hline \multicolumn{5}{|l|}{ Net family income per month $b$} \\
\hline$€ 0-500$ & $1(1.8)$ & $3(6.5)$ & $1(2.1)$ & $5(3.4)$ \\
\hline$€ 500-835$ & $11(20.0)$ & $14(30.4)$ & $11(22.9)$ & $36(24.2)$ \\
\hline$€ 835-1100$ & $18(32.7)$ & $8(17.4)$ & $9(18.8)$ & $35(23.4)$ \\
\hline$€ 1100-1665$ & $17(30.9)$ & $15(32.6)$ & $16(33.3)$ & $48(32.2)$ \\
\hline$€ 1665-2500$ & $5(9.1)$ & $3(6.5)$ & $6(12.5)$ & $14(9.4)$ \\
\hline$\geq € 2500$ & $3(5.5)$ & $3(6.5)$ & $5(10.4)$ & $11(7.4)$ \\
\hline \multicolumn{5}{|l|}{ Previous depressive symptoms } \\
\hline No & $7(12.7)$ & $12(26.1)$ & $12(25.0)$ & $31(20.8)$ \\
\hline Yes & $48(87.3)$ & $34(73.9)$ & $36(75.0)$ & $118(79.2)$ \\
\hline \multicolumn{5}{|l|}{ Current treatment for symptoms } \\
\hline No & $34(61.8)$ & $31(67.4)$ & $36(75.0)$ & $101(67.8)$ \\
\hline Yes & $21(38.2)$ & $15(32.6)$ & $12(25.0)$ & $48(32.2)$ \\
\hline \multicolumn{5}{|l|}{ Current exercise activities } \\
\hline No & $36(65.5)$ & $27(58.7)$ & $32(66.7)$ & $95(63.8)$ \\
\hline Yes & $19(34.5)$ & $19(41.3)$ & $16(33.3)$ & $54(36.2)$ \\
\hline No. of Life events * & $11.35(S D=9.59)$ & $16.70(S D=16.51)$ & $12.35(S D=6.53)$ & $13.32(S D=11.63)$ \\
\hline $\begin{array}{l}\text { Intervention adherence } \\
\text { (No. of sessions attended) }\end{array}$ & $5.47(S D=2.63)$ & $4.57(S D=2.94)$ & -- & $5.06(S D=2.79)$ \\
\hline
\end{tabular}

Continuous data: mean (standard deviation); categorical data: frequency (percentage) $\mathrm{EP}=$ exercise and psycho-education; $\mathrm{E}=$ exercise only; $\mathrm{WLC}=$ waiting-list control

a Educational level: low $\leq 9$ years; $\mathrm{mid}=10$ to 14 years; high $\geq 15$ years.

b The average net family monthly income in the Netherlands in 2007 was $€ 1743$ ${ }^{*} p<0.05$ 


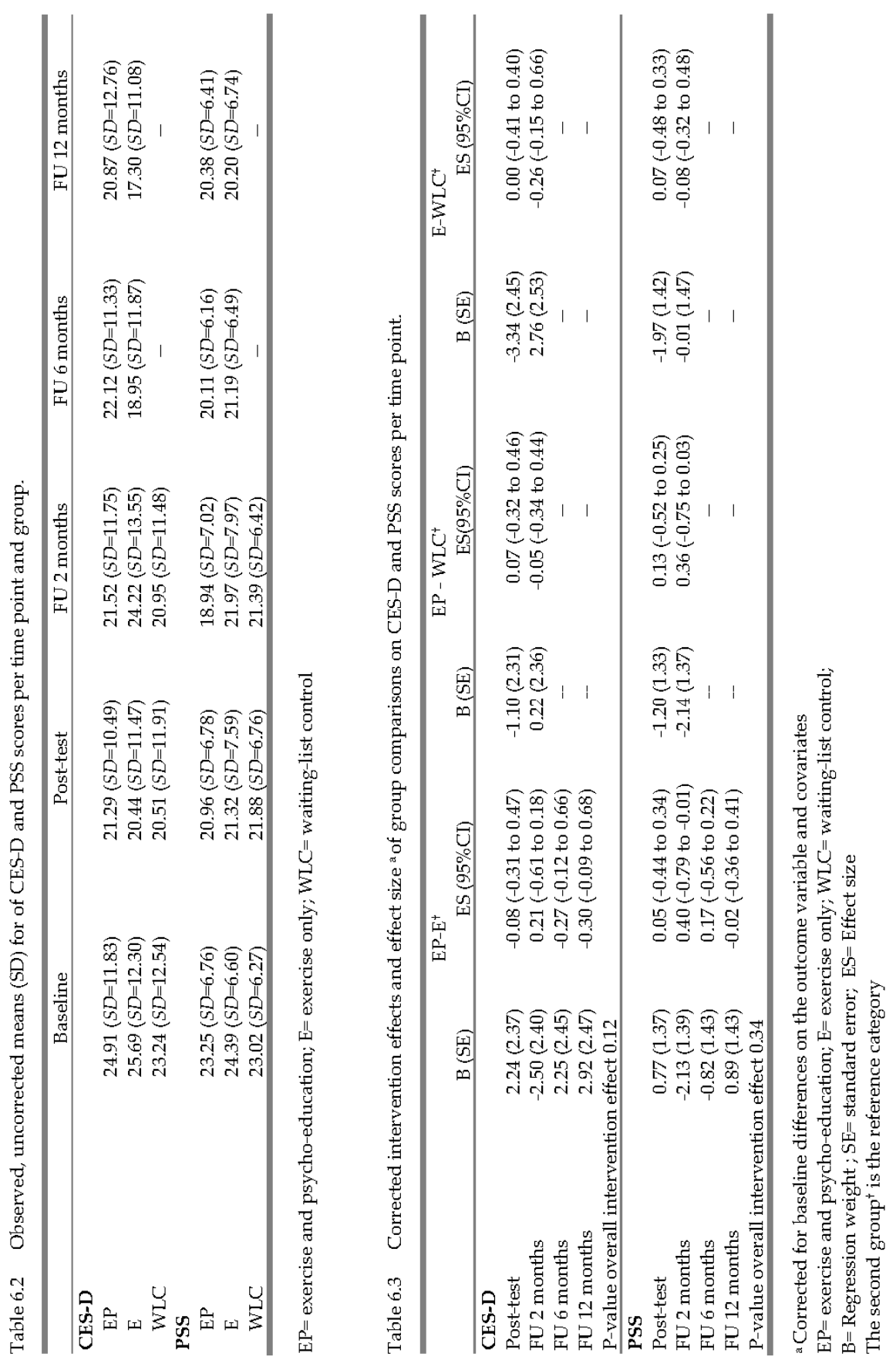




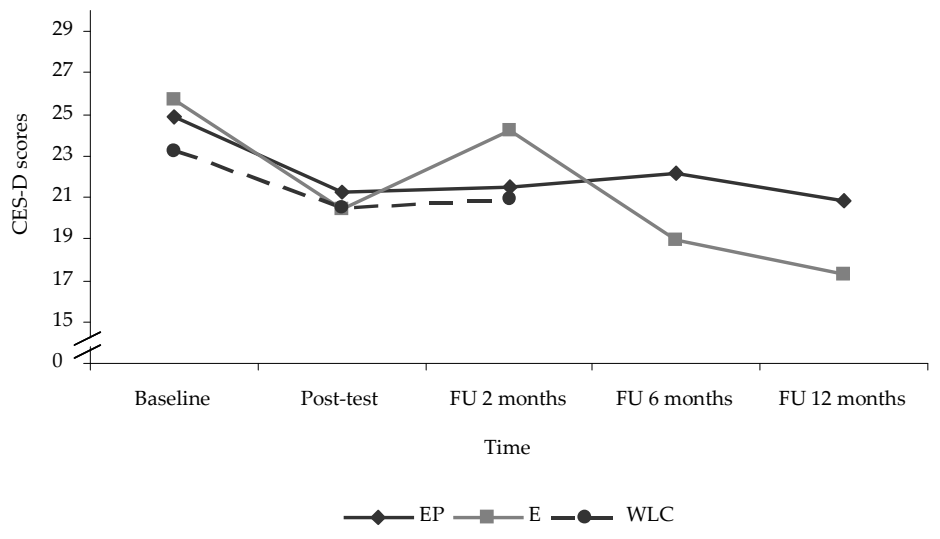

Figure 6.2 Patterns of CES-D scores per time point and group.

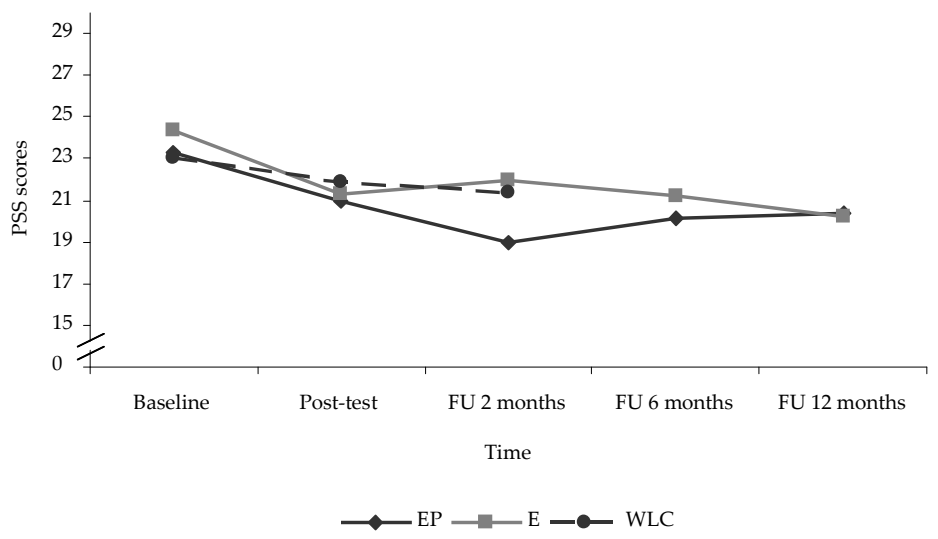

Figure 6.3 Patterns of PSS scores per time point and group.

\section{Intervention effects in subgroups}

The moderation analyses showed intervention effects in some subgroups. For depressive symptoms, women in the EP and E conditions with a CES-D baseline score below the 16-point cut-off score reported significantly fewer depressive symptoms at 2 months FU ( $\beta_{\mathrm{EP}}=-7.77, p=0.01, E S=0.71 ; \beta_{\mathrm{E}}=-7.28$, $p=0.03, E S=0.63$ ) than women in the WLC condition. Conversely, women in the E condition with a CES-D baseline score above the 16-point cut-off had 
significantly more depressive symptoms at 2 months FU than women in the WLC condition $(\beta \mathrm{E}=8.05, p=0.009, E S=-0.56)$.

Subgroup analyses for the PSS showed that women from the EP and E conditions with the lowest educational level had lower perceived stress scores at post-test than women from the WLC condition $\left(\beta_{\mathrm{EP}}=-4.12, p=0.024, E S=0.53\right.$; $\beta \mathrm{E}=-4.13, p=0.025, E S=0.35$ ). None of the other conditions by time by subgroup variable interactions was significant for either outcome measure.

\section{Discussion}

This randomized trial evaluated program effects on the reduction of depressive symptoms and perceived stress levels in a sample of low-SES women. It was the first study on the possible benefits of combined exercise and psychoeducation for this population. This has also been the first trial to extend followup measures for possible intervention effects to one year after participation in these types of combined interventions. While women in the combined exercise and psycho-education and the exercise only groups showed significant decreases in their depressive symptoms and perceived stress levels over time, this decrease was not significantly different from what was found in a wait list control group. Nor did the combined exercise and psycho-education group outperform the exercise only condition on either outcome. Our findings are largely consistent with the limited number of previous reports on the effectiveness of integrated exercise/psychosocial interventions for mental health. These studies did not show a significant additive effect for exercise combined with empirically supported psychosocial approaches compared to exercise alone (Fremont \& Craighead, 1987; McGale et al., 2010). With the current study we have been able to generalize outcomes for these additive effects to a population of low-SES women.

The lack of clear overall intervention effects may be attributable to several factors. First, we assumed that the perceived stress and depressive symptom levels of women in the WLC group would not improve during the four month period before they started the EWW course. The perceived stress and depressive symptom scores of the WLC group as a whole did improve, although this was to a lesser extent than the intervention groups. In their metaanalysis of waiting list controls, Posternak and Miller (2001) suggested that the effect of seeking treatment and obtaining a thorough evaluation in the context of research in itself may very well cause an increased remission rate in depressive symptomatology. However, while this may true for treatment studies, there is no evidence to show whether this effect of treatment seeking 
applies for prevention. Participants in preventive interventions may have lower initial levels of mental distress than those seeking treatment (Cuijpers, Smit, \& Van Straten, 2007). Although it has been suggested that the level of spontaneous recovery is not greatly influenced by initial symptom severity (Posternak \& Miller, 2001), it has also been observed that control groups in preventive interventions do not always show a decline in depressive symptomatology (e.g. Allart-van Dam et al., 2003; Clarke et al., 2001). Therefore it remains speculative as to whether a treatment seeking effect occurred. The reduction of depressive symptoms among WLC participants may well reflect regression to the mean, as improvements can occur in a population that already has increased depressive symptoms and stress levels at enrollment in the study.

A second factor to take in consideration is the intervention format and content. Previous research has indicated that in the Netherlands low-SES groups drop out more frequently from psycho-educative interventions like the Coping With Depression course due to the cognitive orientation and written homework assignments included in these courses (Allart-van Dam \& Hosman, 2002). The EWW course tried to meet these concerns by adapting its course contents to a low-SES target group. This included, among others measures, extending the CWD contents with topics related to risk factors for low-SES women (such as mastery and rumination) and removing barriers to facilitate adherence. In addition, the course contents were tailored to the skills of the target group and there were no homework assignments. Finally, the exercise component was added. The EWW course was also designed to coordinate the psycho-educative subjects with body focused exercises as far as possible. By doing so, we anticipated that the different topics would be reciprocally reinforced. However, given the lack of an overall effect, the eight-week intervention may have been too short to exert the full physiological or psychological benefits for this disadvantaged population. A previous metaanalysis has indicated that depression prevention interventions with 8 sessions or more were more effective than shorter interventions (Jané-Llopis et al., 2003). The CWD course included 12 to 15 sessions, which is similar to the duration of most other programs that are aimed at preventing depression and that have shown evidence of efficacy. See, for instance, Cuijpers et al. (2005), Munoz et al. (2007), Clarke et al. (2001). Compared to these effective interventions, the EWW course covered more topics in fewer sessions. Participants' knowledge of intervention themes may have remained too limited to effectively counter the structural stressors and problems present in their daily lives, and a longer intervention may well have been needed for them to internalize this content. Adherence data for the EWW intervention show that 
women attended on average 5 out of 8 sessions. Although it is possible that this low participation further decreased the intervention's effect, adherence was not found to moderate the effects.

Generally, the proposed explanatory factors for exercise on depressive symptom reduction include physiological, psychological and social support mechanisms (Paluska \& Schwenk, 2000; Stathopoulou et al., 2006). It has been suggested that physical activity can contribute to beneficial effects on depressive symptomatology when it is executed in the amount of the recommended PHD (Dunn et al., 2005). The exercise component in the EWW course, however, did not meet the PHD, which is at least 150 minutes of moderate physical active throughout the week (WHO, 2010). Thus it is unlikely that this intervention activated any physiological mechanisms associated with the reduction of depressive symptoms. Still, the exercise component in the intervention was meant to activate the psychological and social support mechanisms. However, the intervention dose may not have been sufficient to active these mechanisms either. In general, more knowledge is needed on the minimal dosage of exercise needed to produce preventive effects in depression symptomatology (Dunn et al., 2005). The same applies to the amount of education needed for each of the themes in the psycho-educative part, including the use of homework.

Thirdly, we assumed that the group based format could reduce levels of perceived stress and depressive symptoms by increasing social support among the low-SES participants and giving them the chance to share their experiences. However, this might have had adverse effects as well. Some studies indicate that social networks might serve as a conduit for stress among disadvantaged women, since members of these social networks are themselves likely to be poor and stressed (Belle \& Doucet, 2003). Negative social interaction can diminish the protective effects of support and reduce wellbeing (Bassuk, Mickelson, Bissell, \& Perloff, 2002).

In a few subgroups of low-SES women we did find an effect for the EWW intervention. In the short term, women from the EP and E conditions with moderate baseline depression levels significantly outperformed the WLC group. These outcomes concur with previous research on the prevention of depression. A trial by Allart et al. (2003) showed that the positive effects of the intervention were exclusively confined to participants with low to moderate pre-test depression levels. This repeated finding suggests that the current intervention might be adequate for low-SES women with moderately increased levels of depressive complaints, but might not be long or intensive enough for those with higher symptom levels. 
In addition, both the EP and E conditions showed post-intervention effects in reducing perceived levels of stress among women with the lowest educational level. Previous research has shown that this group is generally more affected by the negative impact of chronic stressors related to a low-SES status than women with a higher education (Stronks, Van de Mheen, Looman, \& Mackenbach, 1998). Even though women only followed 5 sessions on average, their participation still resulted in reduced feelings of stress. Lower educated women may be less familiar with the intervention topics (e.g. relaxation exercises) than their higher educated counterparts living in disadvantaged neighborhoods, and may thus have profited more from participation. Previous studies have documented the beneficial effects of exercise on stress responsivity (Salmon, 2001; Steptoe, Kimbell, \& Basford, 1998). Since women in both the EP and E conditions showed reduced perceived stress levels, the exercise itself may also have been an important mechanism for improved mental health outcomes.

While women maintained their reduced perceived stress and depressive symptom levels at 6 and 12 months FU, the levels of the combined group were equal to the exercise only group. Since we did not study long-term outcomes for the WLC, we cannot infer that this long-term reduction on both outcomes is attributable to the EWW intervention.

The strengths of the present study were its focus on a study population of low-SES women, who are generally considered to be hard to reach for participation in prevention and research trials, and the length of the follow-up period (Gadalla, 2008). Some limitations should be mentioned as well. Firstly, for ethical reasons, we did not use a control condition across the whole study period. Our study design allowed for the evaluation of the effects relative to a WLC group immediately after the intervention and at 2 months FU. However, WLC participants were free after these first two months to enter the intervention and thus could not provide data to test long-term follow-up of intervention effects. Also we did not monitor whether participants in the WLC participated in additional preventive activities or sought professional help elsewhere during their wait. If they had done so, this could help to explain their improved short-term outcomes. Secondly, the effect sizes for study group comparisons across several time points (ranging from $d=0.00$ to $d=0.29$ ) were smaller than anticipated at the a priori power calculation $(d=0.40)$. This together with some attrition of participants before baseline measurement may have resulted in a type II error indicating that the present study did not have enough power to detect significant differences with small effect sizes. 
Thirdly, at the 12 month FU women in the E condition appeared to have experienced significantly more life events during the study period than women from the other conditions. We corrected for this post hoc selective randomization by including the number of life events as a covariate in the analyses. However, it is possible that this difference in number of life events did account for some of our overall lack of results (Rochon, 1999).

Fourth, the results relied on self-report questionnaires that, because of their length and level of difficulty, could have posed problems to a low-SES population. We tried to accommodate response by having a trained interviewer to administer the questionnaires. This, in turn, could have caused participants to give socially desirable responses (Bowling, 2005). Another related factor could have been the interview setting. All measurements were conducted face-to-face, except the 2 month FU, which was conducted by phone. Especially in the $\mathrm{E}$ condition, there was an increase of reported symptoms at this measurement. Administering the questionnaires by phone may have led to higher scores. However, studies report no apparent differences in responses to the CES-D between telephone and face-to-face interview methods (Aneshensel, Frerichs, Clark, \& Yokopenic, 1982; Aquilino, 1998), or when they have, phone respondents have reported fewer rather than more symptoms (Chan, Orlando, Ghosh-Dastidar, Duan, \& Sherbourne, 2004).

Finally, perceived stress and depressive symptoms are generally considered as the last link in a chain of effects (Aneshensel, 2009), in which proximal factors such as rumination and lack of social support are related to an increased risk for these mental health problems (McLeod \& Nonnemaker, 1999; Sterk, Theall, \& Elifson, 2006). It is conceivable that the EWW intervention led to significant changes in these proximal variables, since these were explicitly targeted during the course. Thus, the next step for us will be to evaluate these proximal outcome variables and determine whether they mediate intervention effects on stress and depressive symptom outcomes.

In this study, a 'hard to reach' population of low-SES women participated in an exercise intervention. A process evaluation showed that the participants highly appreciated the intervention organization and contents and reported a subjective reduction in their stress and depressive symptoms after 8 sessions (Van der Waerden, Jansen, Hoefnagels, \& Hosman, in press). Like previous reports, the current study has not been able to give unequivocal support for the integration of exercise with psychosocial approaches to benefit mental health (Fremont \& Craighead, 1987; McGale et al., 2010). The EWW intervention was not found to reduce complaints in its disadvantaged target group more than exercise alone. However, in light of the limitations of this study, it would be 
premature to conclude that combining exercise and psycho-education has no merit. This study is the first efficacy trial conducted on this newly developed program. Further program improvements might be needed, for both the exercise and the psycho-educative components, before definitive conclusions can be made on effectiveness. Taken into account the attractiveness of a nonstigmatizing course for disadvantaged women and the meta-analytic outcomes on other efficacy studies (Jané-Llopis et al., 2003) a new trial would be advised using the EWW program with a higher dosage and a longer duration for both intervention components, enhancing also the potential of sustainable social support. Although adherence was reasonable, it could be further improved by using motivational techniques. Previous research has indicated that interviews can reduce the barriers that there are among low-SES women to use mental health care service and can encourage potential participants to commit to an intervention (Grote, Zuckoff, Swartz, Bledsoe, \& Geibel, 2007). Finally, given the outcomes of our subgroup analyses, we recommended that this intervention be specifically targeted at low-SES women with moderate risk levels. This suggests that the EWW intervention could well be implemented as part of a stepped-care approach (Bower \& Gilbody, 2005), in which all participants start with a low-intensity treatment. Symptoms are monitored carefully and participants who fail to respond or exhibit insufficient signs of recovery can subsequently 'step up' the treatment intensity (Meeuwissen, Van der Feltz-Cornelis, Van Marwijk, Rijnders, \& Donker, 2008). Given these considerations, coupled with the notable burden of depression among low-SES women and the observation that this gap in socio-economic health inequalities continues to increase (Dalstra, Kunst, Geurts, Frenken, \& Mackenbach, 2002), the study of viable prevention options among this high-risk group should remain a priority. 


\section{Chapter 7}

Targeting psychosocial mechanisms with a combined exercise and psycho-educative prevention program for low-SES women:

Outcomes of a randomized trial

Judith E.B. van der Waerden, Cees Hoefnagels, Maria W.J. Jansen, and Clemens M.H. Hosman

Submitted for publication 


\section{Abstract}

\section{Background}

The Exercise without Worries intervention aims to reduce stress and depressive symptoms in low-SES women by means of an eight-week group based program combining physical exercise and psycho-education. Although an RCT did not indicate that the Exercise without Worries course reduced depressive symptoms and stress levels in disadvantaged women more than the comparison conditions, the effects of the Exercise without Worries course on psychosocial program outcomes have not yet been determined.

\section{Methods}

A total of 161 low-SES women with elevated stress or depressive symptom levels were randomly assigned to either the combined exercise/psycho-education intervention (EP, $n=57)$, exercise only $(E, n=51)$ or a waiting list control condition $(W L C, n=53)$. General self-efficacy (ALCOS), negative self-statements (ATQ-N), rumination (IBI), self-esteem (NPV-ZW), mastery (PMS), and social support (SSL-I; SSL-D) were measured before and immediately after the intervention and at 6 and 12 months follow-up.

\section{Results}

Multilevel linear mixed-effects models showed significant within-group improvements for the respondents in both of the experimental conditions on various psychosocial outcomes. However these findings did not differ from the changes in the WLC group immediately after the intervention.

\section{Conclusion}

Overall, outcomes suggest the Exercise without Worries course for low-SES women did not have any beneficial effects on important psychosocial resources associated with risk for depression in this group. A longitudinal explorative study of the associations between the targeted psychosocial factors on the one hand and stress and depression on the other could give more indications of which outcomes should be explicitly targeted in preventive interventions for low-SES women. 


\section{Introduction}

Women with a low socio-economic status (SES) have an elevated risk for depression compared to the general population (Bassuk, Buckner, Perloff, \& Bassuk, 1998; Belle \& Doucet, 2003). The multiple pathways through which the ongoing stress of a low social status may affect mental health outcomes have been elaborated on in the Stress Process Model proposed by Paerlin and colleagues (1989; 1999; 1981). Higher rates of depressive symptomatology in low-SES women are moderated through elevated levels of psychological distress associated with the disproportionate amount of both daily hassles and chronic stressors linked to their disadvantaged social position (Grzywacz, Almeida, Neupert, \& Ettner, 2004). At the same time, disadvantaged populations have fewer and less adequate personal and social resources to cope with this increased psychological distress (Denton, Prus, \& Walters, 2004; Elliott, 2001; Ennis, Hobfoll, \& Schroder, 2000; Miranda \& Green, 1999). Less effective use of coping strategies, a low sense of mastery over life, reduced selfesteem and a lack of social support may all lead to diminished resilience and detrimental mental health outcomes (Bassuk, Mickelson, Bissell, \& Perloff, 2002; Roman et al., 2009; Sjögren, Kristenson, \& Linquestgroup, 2006).

Some selective and indicated interventions have been specifically designed to prevent depression in low-SES women and offer opportunities to promote effective use of personal and social resources (Cardemil, Kim, Pinedo, \& Miller, 2005). These interventions apply a variety of methods but mostly use psychosocial and educational techniques (Levy \& O'Hara, 2010; Van der Waerden, Hoefnagels, \& Hosman, 2010). In recent years evidence has increased for the beneficial impact of exercise in reducing depressive symptoms and stress (Alderman, Rogers, Johnson, \& Landers, 2003; Stathopoulou, Powers, Berry, Smits, \& Otto, 2006). While the research on the combination of exercise with empirically supported psychosocial approaches for depression has been limited (Stathopoulou et al., 2006), exercise is increasingly accepted as an adjunct intervention in the management of depressive symptoms and depression (Fremont \& Craighead, 1987; McGale, McArdle, \& Gaffney, 2010; Merom et al., 2008).

To this effect, the Exercise without Worries (EWW) course was developed and evaluated in the Netherlands combining an exercise regimen with a cognitive behavioral therapy (CBT) approach specifically targeted at low-SES women. The EWW course aimed to reduce stress and depressive symptoms in this group by means of an eight-week group based program (Van der Waerden, Jansen, Hoefnagels, \& Hosman, in press). Through a combination of physical exercise and psycho-education, the EWW course targeted risk factors 
relevant for low-SES women. The development of the intervention was guided by the Stress Process Model of mental health (Pearlin, 1989; Pearlin, Menaghan, Lieberman, \& Mullan, 1981). The psycho-education addresses risk factors for depression such as negative thinking, social skills, self-esteem, and promoting resources to counter rumination. These topics have been extended with other evidence-based risk factors for stress and depression specific for the target group of low-SES women, such as low self-esteem and low perceived mastery over life (Lanen, Gelissen, Ebben, \& Van der Waerden, 2008). The exercise component employs stretching, muscle reinforcement, flexibility, body focused exercise and relaxation. The hypothesized mechanisms for the psychological benefits of exercise include distraction from unpleasant thoughts, improved self-efficacy and self-esteem, behavioral activation, mastery, and selfdetermination (Fox, 2000; Hassmén, Koivula, \& Uutela, 2000; Penedo \& Dahn, 2005; Scully, Kremer, Meade, Graham, \& Dudgeon, 1998). Social interaction and social support from other participants were encouraged through the group based format, and also through the exercise component in which participants exercise together (Azocar, Miranda, \& Dwyer, 1996; Brosse, Sheets, Lett, \& Blumenthal, 2002; Paluska \& Schwenk, 2000).

When developing the EWW intervention, we assumed that combining different types of effective interventions would increase and prolong the positive effects of both (Tsang, Chan, \& Cheung, 2008). However, the randomized trial showed no evidence that the EWW course reduced depressive symptom and stress levels in women from low-SES neighborhoods more than the comparison conditions. Examining effects on psychosocial outcomes may be an important step to further identifying if and how this intervention might work. It is conceivable that the EWW intervention did lead to changes in these mechanisms, since most of these psychosocial factors were explicitly targeted during this course.

Thus, the aim of the current study was to evaluate the effects of the EWW course on psychosocial program outcomes. We compared whether the combination of exercise and psycho-education (EP) leads to synergetic effects on participants' mental wellbeing, contrasted to those participants receiving exercise only (E) and to a waiting list control (WLC) condition. Our hypothesis is that women in the EP and $\mathrm{E}$ conditions show greater improvements on psychosocial outcomes than those in the WLC group. Also we expected participants in the EP condition to show a larger improvement on psychosocial outcomes than those in the E condition since these outcomes are possibly stimulated through both exercise and psycho-education. 


\section{Method}

\section{Study design and participants}

The efficacy of the EWW course was tested in a randomized control trial. The target population of the EWW intervention consisted of low-SES women (20-55 years) living in disadvantaged neighborhoods with depressive symptoms or stress-related complaints. Between April 2005 and November 2007, participants were recruited through general practitioners active in or nearby socioeconomically deprived neighborhoods, social work, debt reform, the community mental health center and public health services and local media campaigns. Women referred to the EWW course completed a 10-minute telephone interview conducted by trained lay interviewers to screen them for the presence of stress and depressive complaints. Women who satisfied lowSES criteria, had stress or depression scores above predetermined cut-off levels, and were interested in participating in the EWW were invited to an intake. During this intake, the course objectives were explained and further background information related to the women's complaints and functional status was collected. Women were excluded from participation in the course if their Dutch was limited or if they had cognitive disturbances or emotional instability that might impede participation in a group intervention or physical problems that might hinder their participation in the exercise component of the course. A more detailed description of the recruitment process and willingness of low-SES women to participate in the EWW intervention is described elsewhere (Van der Waerden, Hoefnagels, Jansen, \& Hosman, 2010).

Those women suitable and willing to participate in the RCT signed a written informed consent form for participation and were subsequently randomly assigned using a randomization list. Participants were assigned to one of three intervention conditions: the full EWW intervention with exercise and psycho-education (EP) component, the intervention with exercise only (E) component or a waiting list control (WLC) condition with postponed intervention. The study design did not include a psycho-education only condition $(\mathrm{P})$, since this component has already been shown to be evidencebased in the reduction of depressive symptoms (e.g. Cuijpers, Munoz, Clarke, \& Lewinsohn, 2009; Cuijpers, Smit, \& van Straten, 2007). Until the first session, participants were blinded to the treatment group to which they were assigned. Measurements in the EP and E conditions were performed at baseline, post-test directly after the eight-week intervention, and 6 and 12 months follow-up (FU). Participants in the WLC group completed baseline and post-test measurements at the same time as their experimental condition counterparts. Four months after baseline, they were given the opportunity to participate in the 
intervention. During the study period, 161 eligible women gave informed consent and were randomly allocated to the EP, E and WLC groups (Figure 7.1). Approval for conducting this study was granted by the Medical Review Ethics Committee of the Academic Hospital Maastricht/Maastricht University in the Netherlands.

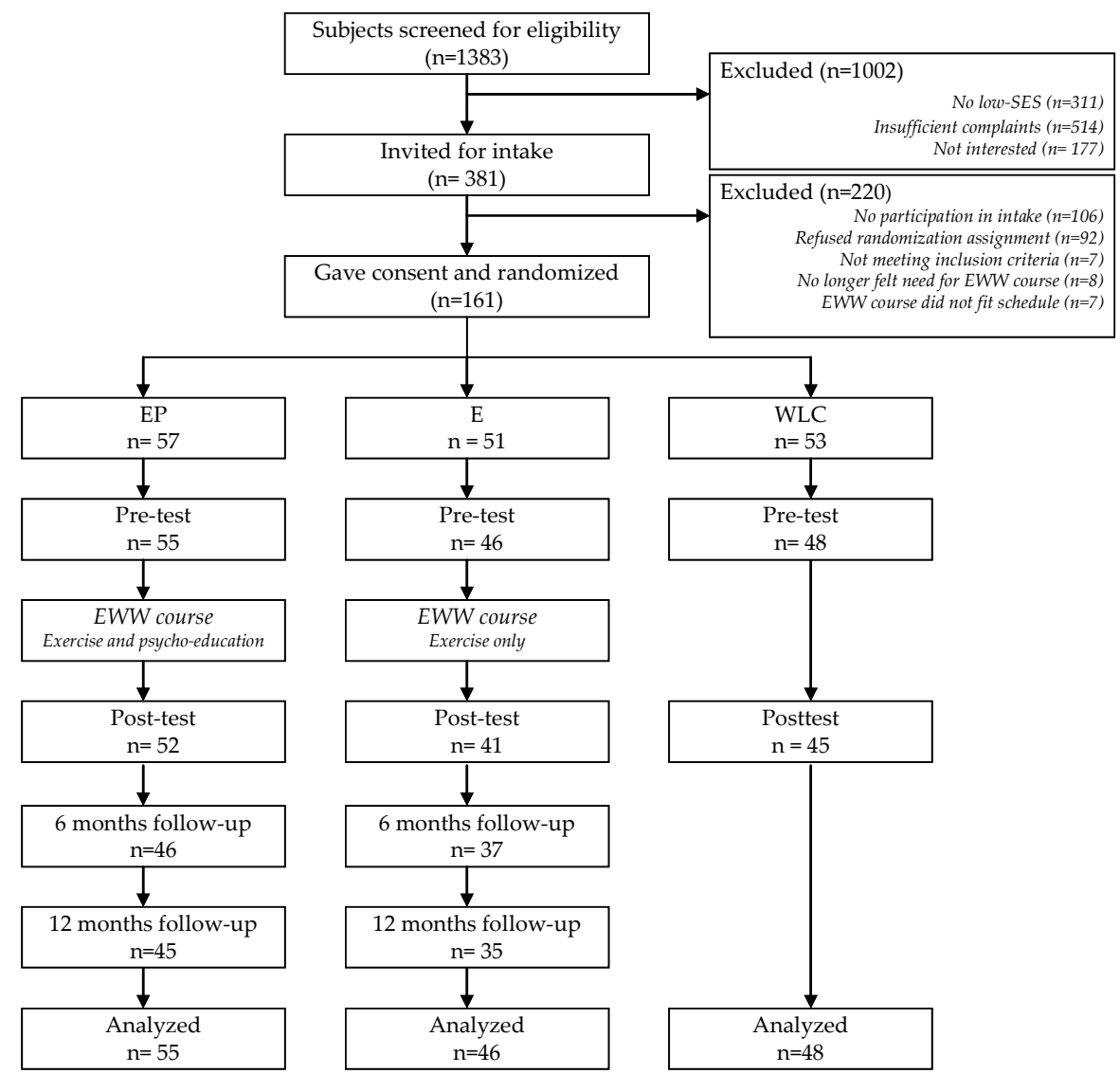

$E P=$ exercise and psycho-education; $E=$ exercise only; $W L C=$ waiting-list control

Figure 7.1 Flow of the participants through the study

\section{Intervention and comparison group activities}

Exercise without Worries (EWW) is a new preventive course that has been tailored to the specific needs of low-SES women. The intervention's main goal is to reduce stress and depressive complaints and increase coping related competences. The emphasis lies on empowering women instead of focusing on their problems. One of the most important elements in the course is that each 
of the eight two-hour sessions links the psycho-educative subjects with the body focused exercises. The psycho-education and exercise components are coordinated as far as possible with the aim of reciprocally reinforcing the message. Each session was led by two trainers, a licensed psychologist or mental health provider and an exercise professional such as a physical therapist or licensed sports instructor. These trainers provide positive feedback to enhance participants' self-esteem and confidence. A more comprehensive description of the EWW course has been reported elsewhere (Lanen et al., 2008).

The E condition included the same exercises as the combined program, but without the psycho-educative component. During the exercise only sessions, the refreshment breaks were extended to correspond with the time provided for staff-client contact in the EP course. Finally, the women in the WLC condition did not participate in the intervention until four months later and were instructed to continue life as normal and were free to engage in both physical activity and mental health counseling elsewhere.

\section{Measures}

\section{Personal resources}

General self-efficacy was measured with the Dutch version of the General SelfEfficacy Scale (Algemene Competentie Schaal, ALCOS) (Bosscher, Smit, \& Kempen, 1997; Sherer et al., 1982). This scale describes a person's belief in his or her ability to cope with a broad range of stressful or challenging demands. The ALCOS consists of 16 items rated on a five-point scale ranging from 1 (strongly agree) to 5 (strongly disagree). A high score indicates high general self-efficacy (Bosscher, Van der Aa, Van Dasler, Deeg, \& Smit, 1995). Internal consistency and reliability of the ALCOS appear to be good (Bosscher \& Baardman, 1989). For this sample, reliability across the different time points was sufficient with Cronbach's $\alpha$ between 0.70 and 0.78 .

The Automatic Thoughts Questionnaire-Negative (ATQN) (Hollon \& Kendall, 1980) is a 30-item questionnaire designed to measure the frequency of negative self statements associated with depression. The original version of the ATQ had a good reliability and is able to discriminate between people with and people without depression (Hill, Oei, \& Hill, 1989; Hollon \& Kendall, 1980). Cronbach's $\alpha$ in the present study was 0.97 for all consecutive time points.

To measure rumination, the worrying subscale of the Irrational Beliefs Inventory (IBI) (Koopmans, Sanderman, Timmerman, \& Emmelkamp, 1994) was used. The 12 items on this subscale measure how much people worrying 
about possible future accidents and misfortune. The respondent evaluates each of the items on a five-point scale ranging from 1 (strongly agree) to 5 (strongly disagree). Reliability for this subscale has been found to be high, with a Cronbach's $\alpha$ between 0.79 to 0.84 for respondents of different nationalities (Bridges \& Sanderman, 2002). Cronbach's $\alpha$ in the present study ranged between 0.78 and 0.84 across the data points.

Self-Esteem was assessed with the self-esteem subscale of the Dutch Personality Questionnaire (Nederlandse Persoonlijkheids Vragenlijst, NPV-ZW) (Luteijn, Starren, \& van Dijk, 1985). This subscale measures people's positive attitude towards themselves and is a 19-item true/false self-report questionnaire. In other studies, the median Cronbach's $\alpha$ for the self-esteem scale is 0.74 (Luteijn et al., 1985). Cronbach's $\alpha$ in the present study was between 0.77 and 0.82 .

To assess mastery or perceived control of participants, the Pearlin Mastery Scale was applied (PMS) (Pearlin et al., 1981; Pearlin \& Schooler, 1978 ). This 7item scale measures the extent to which individuals perceive themselves in control of forces that significantly impact their lives. Items are rated from 1 (strongly disagree) to 4 (strongly agree). The total scores range from 7 to 28 , with higher scores indicating a higher degree of believing oneself to be in control. The PMS has been shown to exhibit reasonable internal reliability (Seeman, 1991) and good construct validity (Pearlin et al., 1981). Reliability in the present study ranged between Cronbach's $\alpha 0.68$ and 0.82 across different measurement points.

\section{Social resources}

Social support was measured with the short version of the Social Support List (SSL), which has shown good construct validity and high reliability (Timmerman, Emanuels-Zuurveen, \& Emmelkamp, 2000; Van Sonderen, 1993). For this study, we investigated both the number of supportive interactions that people receive (SSL-I), as well as the discrepancies (SSL-D) that people experience in receiving social support from their direct environment. The SSL-I measures the amount of supportive interactions people receive from their social support network. A total score was measured by summing up the scores on the 12 SSL-I items; the higher the score the more social support a person received (Cronbach's $\alpha$ across measurements was between 0.88 and 0.92). The SSL-D assesses the extent to which the support a person received is in accordance with their needs. The outcomes represent the subjective experience of mismatches between the received and desired frequency of social support. After recoding items in the same direction, the scores of the 12 items were added to a total SSL-D score. A higher score on the SSL-D scale reflects greater 
dissatisfaction with the social support that someone has experienced. The reliability of the SSL-D in our sample was good (Cronbach's $\alpha$ between 0.88 and 0.93).

The following socio-demographic variables were determined at baseline: age, nationality, educational level, and previous depressive episodes. Employment status, net monthly family income, marital status, current depression treatment and current exercise behaviors were established at baseline, as well as during each subsequent time point. At 12 months FU, participants also completed the Dutch Recent Life Events Questionnaire (RLEQ), which counts the total number of major life events experienced over the past 12 months (Willige, Schreurs, Tellingen, \& Zwart, 1985). The Cronbach's $\alpha$ in this sample was 0.89 . All questionnaires were administered at participants' homes by trained interviewers to assure maximum response from the target group (Picavet, 2001).

\section{Data analysis}

The data analysis included descriptive statistics of the socio-demographic and other background variables and primary outcome measures. Women were included in the analyses if they had completed the baseline assessment. Dropout analyses were conducted using logistic regression analyses to explore differences in socio-demographic characteristics for those participants who did not complete all measurements after the baseline assessment. To check for preexisting differences among the three conditions on descriptive variables and outcome measures at study entry despite randomization, an analysis of variance (ANOVA) was carried out for continuous variables and Chi-square analyses were performed for categorical variables.

Since participants were nested within EWW course groups and participated in multiple measurements, multilevel linear regression analyses were used to account for dependency in the consecutive outcomes (Snijders \& Bosker, 1999). A multilevel approach with random intercept for three levels (course group, participant and time) was completed to analyze the changes in outcome variables between intervention conditions. Separate analyses were performed for each of the outcome measures. Multilevel analyses have also been shown to be useful in handing missing data, since analyses can be done on available cases instead of complete cases only (Twisk, 2006). For all respondents with no more than $20 \%$ of missing values on a given scale and time point, the missing values were substituted by the respondent's mean score on that particular scale (Moons, Donders, Stijnen, \& Harrell, 2006). There were no scales with more than $20 \%$ of missing values; the maximum percentage of missing values for a given scale was for the RLEQ, with $13 \%$ missing. 
To test the change in outcome on the different measures between the intervention conditions, a fixed occasions model was fitted for each outcome variable, with a random group effect and an unspecified $4 * 4$ covariance matrix for participant effects at all four time points. Included predictors were intervention condition variables coded into two dummies (using the WLC as a reference), time of measurement which was recoded into three dummies (with baseline as reference time point), and six two-way interaction terms for each combination of intervention condition*time to test whether the intervention effect varied over time. Baseline socio-demographic variables, baseline scores for the respective outcome measures, intervention adherence, current depression treatment and number of life events were included as betweensubject covariates because these variables were assumed to be related to intervention outcomes. By including these covariates, the power and precision of the testing and estimation of the treatment effect are improved due to reduced residual outcome variance.

The overall intervention effects, and those at post-test, 6 and 12 months FU, were analyzed by following a 'top-down' procedure, starting with the most elaborate model and successively leaving out the least significant components (using alpha $=0.10$ to prevent type II errors). In the most reduced model, which contained time and group by time effects plus remaining significant covariates, the intervention effects were statistically tested using the likelihood ratio test with a 0.05 significance cut-off point for regression coefficients. In all of the analyses, a correction was made for baseline scores, as well as for number of life events. All analyses were performed using PASW Statistics (version 17.0).

The intervention effect sizes were determined using Cohen's method (Cohen, 1988) by dividing the difference between two means by the pooled SDs for those means. Effect sizes were calculated for each outcome for the effect of the EP and E groups compared to the WLC group and between EP and E groups. Effect sizes were interpreted as small when they were equal to or less than 0.32 , medium when they were between 0.33 and 0.55 , and large when they were equal to or greater than 0.56 (Lipsey \& Wilson, 2001).

\section{Results}

Of the 161 women enrolled in the study, 12 dropped out before the baseline assessment took place and were excluded from any further analyses. The participants who dropped out after the baseline measurement did not differ from those who completed all assessments $(p>0.05)$. Table 7.1 presents the socio-demographic characteristics, as well as the presence of previous depressive episodes, current depression treatment and current exercise 
behavior at baseline for each of the three study conditions. No significant differences were found between the three conditions for any of these variables, with the exception of nationality. All participants in the WLC group were Dutch, versus $91 \%$ in the EP condition and $84 \%$ in the E condition $\left(\chi^{2}(2)=7.47\right.$, $p=0.02)$. Furthermore, at the 12 month FU women in the E-condition appeared to have experienced significantly more life events during the study period than women from the other conditions $(\mathrm{F}(2,146)=2.97, p<0.05)$. Women attended on average $5.06(\mathrm{SD}=2.79)$ meetings, without any significant differences between the two intervention conditions.

Table 7.1 Baseline characteristics of 149 participants, means (SD), frequencies (\%)

\begin{tabular}{|c|c|c|c|c|}
\hline & $\begin{array}{l}\text { EP group } \\
(n=55)\end{array}$ & $\begin{array}{c}\text { E group } \\
(\mathrm{n}=46)\end{array}$ & $\begin{array}{l}\text { WLC group } \\
(\mathrm{n}=48)\end{array}$ & Total sample \\
\hline Age (years) & $44.68(S D=8.43)$ & $43.06(S D=8.88)$ & $43.87(S D=7.67)$ & $43.90(S D=8.31)$ \\
\hline Nationality (Dutch)* & $50(90.9)$ & $39(84.8)$ & $48(100.0)$ & 137 ( 91.9) \\
\hline \multicolumn{5}{|l|}{ Marital status } \\
\hline Single, Divorced, Widowed & $22(40.0)$ & $26(56.5)$ & $17(35.4)$ & $65(43.6)$ \\
\hline Married, cohabitating & $33(60.0)$ & $20(43.5)$ & $31(64.6)$ & $84(56.4)$ \\
\hline \multicolumn{5}{|l|}{ Educational level } \\
\hline Low & $27(49.1)$ & $28(60.9)$ & $24(50.0)$ & $79(53.0)$ \\
\hline Middle & $21(38.2)$ & $14(30.4)$ & $22(45.8$ & $57(38.3)$ \\
\hline High & $7(12.7)$ & $4(8.7)$ & $2(4.2)$ & $13(8.7)$ \\
\hline \multicolumn{5}{|l|}{ Employment status } \\
\hline Employed & $13(23.6)$ & $13(28.3)$ & $16(33.3)$ & $42(28.2)$ \\
\hline Housewife & $14(25.5)$ & $8(17.4)$ & $10(20.8)$ & $32(21.5)$ \\
\hline Unemployed & $12(21.8)$ & $8(17.4)$ & $4(8.3)$ & $24(16.1)$ \\
\hline Disabled & $16(29.1)$ & $17(37.0)$ & $18(37.5)$ & $51(34.2)$ \\
\hline \multicolumn{5}{|l|}{ Net family income per month } \\
\hline$€ 0-500$ & $1(1.8)$ & $3(6.5)$ & $1(2.1)$ & $5(3.4)$ \\
\hline$€ 500-835$ & $11(20.0)$ & $14(30.4)$ & $11(22.9)$ & $36(24.2)$ \\
\hline$€ 835-1100$ & $18(32.7)$ & $8(17.4)$ & $9(18.8)$ & $35(23.4)$ \\
\hline$€ 1100-1665$ & $17(30.9)$ & $15(32.6)$ & $16(33.3)$ & $48(32.2)$ \\
\hline$€ 1665-2500$ & $5(9.1)$ & $3(6.5)$ & $6(12.5)$ & $14(9.4)$ \\
\hline$\geq € 2500$ & $3(5.5)$ & $3(6.5)$ & $5(10.4)$ & $11(7.4)$ \\
\hline \multicolumn{5}{|l|}{ Previous depressive symptoms } \\
\hline No & $7(12.7)$ & $12(26.1)$ & $12(25.0)$ & $31(20.8)$ \\
\hline Yes & $48(87.3)$ & $34(73.9)$ & $36(75.0)$ & $118(79.2)$ \\
\hline \multicolumn{5}{|c|}{ Current treatment for symptoms } \\
\hline No & $34(61.8)$ & $31(67.4)$ & $36(75.0)$ & $101(67.8)$ \\
\hline Yes & $21(38.2)$ & $15(32.6)$ & $12(25.0)$ & $48(32.2)$ \\
\hline \multicolumn{5}{|l|}{ Current exercise activities } \\
\hline No & $36(65.5)$ & $27(58.7)$ & $32(66.7)$ & $95(63.8)$ \\
\hline Yes & $19(34.5)$ & $19(41.3)$ & $16(33.3)$ & $54(36.2)$ \\
\hline No. of Life events* & $11.35(S D=9.59)$ & $16.70(S D=16.51)$ & $12.35(S D=6.53)$ & $13.32(S D=11.63)$ \\
\hline $\begin{array}{l}\text { Intervention adherence } \\
\text { (No. of sessions attended) }\end{array}$ & $5.47(S D=2.63)$ & $4.57(S D=2.94)$ & -- & $5.06(S D=2.79)$ \\
\hline
\end{tabular}

Continuous data: mean (standard deviation); categorical data: frequency (percentage). $\mathrm{EP}=$ exercise and psycho-education; $\mathrm{E}=$ exercise only; $\mathrm{WLC}=$ waiting-list control. Educational level: low $\leq 9$ years; mid $=10$ to 14 years; high $\geq 15$ years. ${ }^{*} \mathrm{p}<0.05$. 


\section{Intervention effects}

Table 7.2 shows the descriptive study measures from baseline to one-year FU. Outcomes from the multilevel linear mixed-effects analyses will be discussed for each measure separately.

Table 7.2 Observed, uncorrected means (SD) for personal and social resources outcomes per time point and group.

\begin{tabular}{|c|c|c|c|c|}
\hline & Baseline & Post-test & FU 6 months & FU 12 months \\
\hline \multicolumn{5}{|c|}{ Personal resources } \\
\hline \multicolumn{5}{|c|}{ ALCOS (Self-efficacy) } \\
\hline EP & $47.64(S D=9.49)$ & $45.27(S D=8.84)$ & $45.35(S D=9.43)$ & $44.78(S D=9.33)$ \\
\hline E & $47.98(S D=7.32)$ & $44.78(S D=7.01)$ & $43.98(S D=7.76)$ & $43.35(S D=9.13)$ \\
\hline WLC & $48.87(S D=9.32)$ & $46.60(S D=9.25)$ & -- & -- \\
\hline \multicolumn{5}{|c|}{ ATQ-N (Negative thinking) } \\
\hline $\mathrm{EP}$ & $63.15(S D=24.51)$ & $54.21(S D=19.15)$ & $56.93(S D=19.97)$ & $55.06(S D=24.81)$ \\
\hline $\mathrm{E}$ & $64.38(S D=25.11)$ & $57.49(S D=27.65)$ & $52.90(S D=23.53)$ & $50.84(S D=23.15)$ \\
\hline WLC & $59.08(S D=22.76)$ & $57.87(S D=23.05)$ & -- & -- \\
\hline \multicolumn{5}{|c|}{ IBI (Rumination) } \\
\hline $\mathrm{EP}$ & $42.35(S D=5.91)$ & $40.25(S D=6.62)$ & $39.60(S D=7.31)$ & $38.89(S D=6.99)$ \\
\hline E & $42.61(S D=7.40)$ & $40.48(S D=6.92)$ & $38.68(S D=7.66)$ & $38.76(S D=6.79)$ \\
\hline WLC & $41.85(S D=6.47)$ & $41.38(S D=6.74)$ & -- & -- \\
\hline \multicolumn{5}{|c|}{ NPV (Self-esteem) } \\
\hline $\mathrm{EP}$ & $21.53(S D=7.13)$ & $22.25(S D=6.59)$ & $21.83(S D=7.66)$ & $22.58(S D=6.67)$ \\
\hline E & $20.61(S D=7.12)$ & $22.73(S D=6.61)$ & $24.65(S D=7.16)$ & $24.74(S D=7.09)$ \\
\hline WLC & $20.46(S D=7.84)$ & $20.96(S D=7.69)$ & -- & -- \\
\hline \multicolumn{5}{|c|}{ PMS (Mastery) } \\
\hline $\mathrm{EP}$ & $18.89(S D=3.98)$ & $20.48(S D=4.39)$ & $21.15(S D=4.89)$ & $20.94(S D=5.04)$ \\
\hline E & $18.61(S D=4.37)$ & $20.15(S D=4.60)$ & $21.35(S D=4.44)$ & $22.03(S D=4.90)$ \\
\hline WLC & $20.13(S D=3.98)$ & $20.78(S D=4.40)$ & -- & -- \\
\hline \multicolumn{5}{|c|}{ Social Resources } \\
\hline \multicolumn{5}{|c|}{ SSL-I (Social support) } \\
\hline $\mathrm{EP}$ & $28.04(S D=5.62)$ & $29.12(S D=5.59)$ & $27.63(S D=5.91)$ & $27.16(S D=7.43)$ \\
\hline E & $29.42(S D=6.54)$ & $29.13(S D=7.30)$ & $29.62(S D=6.65)$ & $29.60(S D=6.24)$ \\
\hline WLC & $29.33(S D=6.43)$ & $29.08(S D=5.91)$ & -- & -- \\
\hline \multicolumn{5}{|c|}{ SSL-D (Social support discrepancies) } \\
\hline $\mathrm{EP}$ & $28.09(S D=7.11)$ & $30.92(S D=5.33)$ & $29.64(S D=6.58)$ & $29.44(S D=7.29)$ \\
\hline E & $30.29(S D=6.11)$ & $30.37(S D=7.04)$ & $30.89(S D=6.06)$ & $30.85(S D=5.63)$ \\
\hline WLC & $29.68(S D=6.61)$ & $30.11(S D=6.36)$ & -- & -- \\
\hline
\end{tabular}

$\mathrm{EP}=$ exercise and psycho-education; $\mathrm{E}=$ exercise only; $\mathrm{WLC}=$ waiting-list control

\section{General self-efficacy}

Women in the EP, E and WLC groups showed significant $(p<0.05)$ decreases in self-efficacy at post-test. For the EP and E condition, this change to the pre- 
intervention scores was still significant at the 6 and 12 month FU $(p<0.01)$. However, none of the measurement showed significant differences in selfefficacy scores ( $p=0.87$ ) between the EP, E and WLC groups (see Table 7.3).

\section{Negative self statements}

Compared to their pre-intervention scores, women in the both the EP and E conditions showed a significant decrease in the number of negative self statements at post-test and FU $(p<0.01)$; this was not the case for those in the WLC group. Yet, overall intervention effects on decreases in negative self statements between the three conditions were insignificant on all of the measurements $(p=0.16)$.

\section{Rumination}

Compared to their pre-intervention scores, the women's rumination scores in both the EP and E conditions had decreased significantly at post-test and FU $(p<0.01)$; this was not the case for those in the WLC group. The overall intervention effects on reduced rumination between the three conditions were not significant on any of the measurement moments $(p=0.25)$.

\section{Self-Esteem}

Women in the E, but not the EP and WLC, conditions showed significant $(p<0.01)$ improvements in self-esteem scores at both post-test and FU. While differences between the E and EP group were only marginally significant at post-test $(p=0.07)$ and 12 months $\mathrm{FU}(p=0.06)$, this difference in self-esteem scores was significant at 6 months FU $(p=0.01)$ in favor of women in the E condition. Furthermore, changes in self-esteem in the $\mathrm{E}$ group were significantly different from the WLC group at post-test ( $p=0.03$, see Figure 7.2).

\section{Mastery}

Compared to their pre-intervention scores, women in the both the EP and E conditions, but not the WLC, had significantly higher mastery scores at posttest and FU $(p<0.01)$. Still, the group by time interaction for this outcome was not significant $(p=0.23)$. 


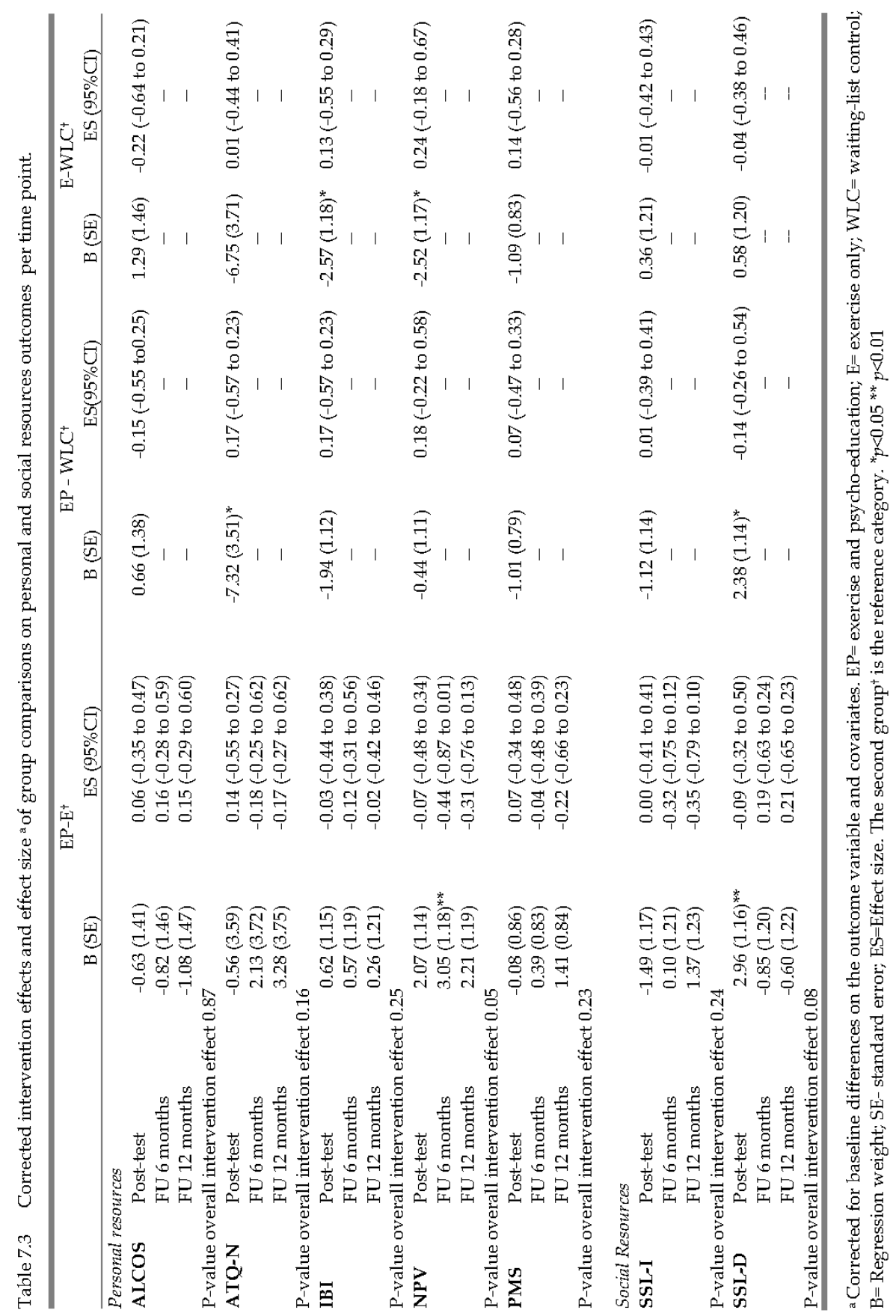




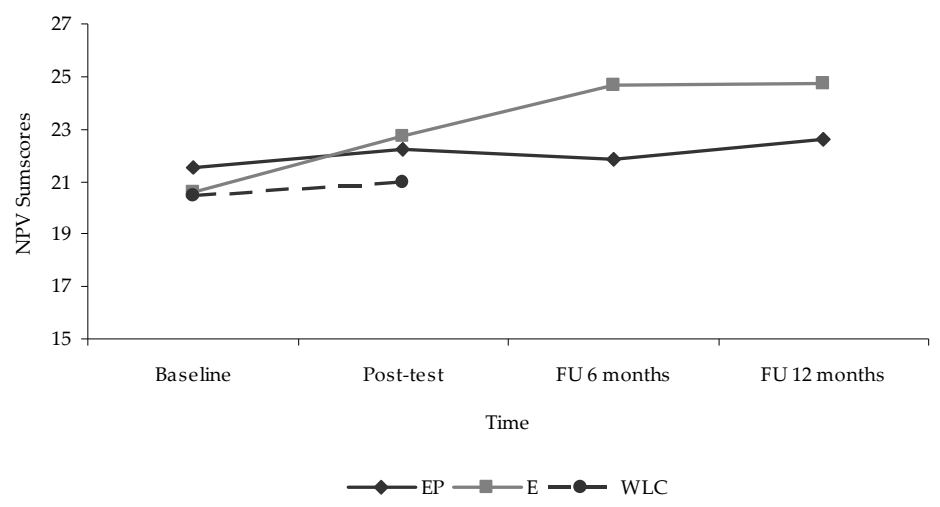

Figure 7.2 Patterns of NPV Self-esteem scores per time point and group.

\section{Social support interactions}

There was no increase is social support for women in the EP, E and WLC groups at either post-test or FU $(p<0.05)$. Neither did the overall intervention effects show significant differences in social support between the three conditions on any of the measurements $(p=0.24)$.

\section{Social support discrepancies}

Outcomes on social support discrepancies indicate that dissatisfaction with social support had increased significantly in the EP condition at post-test. This increase in social support discrepancy was significant compared to changes in the $\mathrm{E}(p=0.01)$ and WLC $(p=0.03)$ groups. However, these group differences were no longer significant at 6 and 12 months FU ( $p>0.05)$.

\section{Discussion}

The Exercise without Worries (EWW) course was developed as a multicomponent intervention that aimed to increase psychosocial resources among its target group of low-SES women. The aim of the intervention was to reduce perceived stress and depressive symptoms in this at-risk population. This study evaluated program effects of the EWW course on several psychosocial outcomes that could reduce risks and bolster resilience in disadvantaged women. 
Overall, outcomes suggest that this preventive intervention did not produce any beneficial effects on important psychosocial resources associated with risk for depression in this group of low-SES women. In addition, no added value was found of a multi-component intervention such as the EWW course. Our first hypothesis was that women in the EP and E conditions would show greater improvements on outcomes than those in the WLC group. Directly after the intervention, as well as 6 and 12 months later, women in the combined exercise and psycho-education and the exercise only groups showed significant improvements in the number of negative self statements, rumination and mastery. Even though these within-group findings showed that the respondents in both of the experimental conditions improved significantly on various psychosocial mechanisms, these findings did not differ from the WLC at post-test. Also, our second hypothesis that participants in the EP condition would show a larger improvement on outcomes than those in the E condition was not confirmed. Women in the combined exercise and psycho-education group did not outperform the exercise only condition on self-efficacy, the number of negative self statements, rumination, or mastery. Additionally, women in the exercise only group showed significantly more improvement in their self-esteem than women in the combined EP condition and those on the waiting list.

While no improvements were found in any of the conditions for the supportive interactions women received from their social support network, participants in the combined EP intervention did report more discrepancies between their needs for social support needs and what they received immediately after the intervention. An explanation could be that sharing their problems with the other course members in the psycho-educative component sensibilized low-SES women to the importance of social support. Network members of disadvantaged women have been found to not always have social support readily available (Bassuk et al., 2002; Belle \& Doucet, 2003). Thus, the intervention could have made the participants more conscious of a mismatch between the amount of social support they habitually receive and what they feel they need. This effect might have been even further exacerbated if the newly found contacts with other group members did not substantiate into a larger social network after completion of the course.

We assumed that the group based format would encourage social interaction and lead to increased perceptions of social support. However, this effect was not found in either the combined intervention or the exercise only condition. Apart from the group setting, neither condition systematically targeted increased social support or boosted social support mechanisms. It has 
been suggested that social mechanisms associated with exercise can have some antidepressive effects (Brosse, Sheets, Lett, \& Blumenthal, 2002; Scully et al., 1998). Even though social interaction may have importance at the beginning of an exercise program, generally the evidence for social mechanisms associated with the effects of physical activity on mental health remains tentative (Paluska \& Schwenk, 2000). These elements considered the lack of effects on social support interactions in this study is not surprising.

Our hypothesis that participants in the EP condition would show a larger improvement on outcomes than those in the E condition was not confirmed. Additionally, women in the exercise only group showed significantly more improvement in their self-esteem than women in the combined EP condition and those on the waiting list. Disadvantaged women characteristically exhibit low self-esteem (Brown, Bifulco, Veiel, \& Andrews, 1990; Nazroo, 2005). Previous studies have indicated that participating in exercise improved selfesteem (Fox, 2000; White, Kendrick, \& Yardley, 2009). However, the combination of psycho-education and exercise did not produce benefits for women participating in the EP condition. Since this group did not show any improvements on self-esteem, the proposed synergetic approach may to some extent have been counterproductive for this outcome.

It should be noted that the E condition did not purely provide exercise. The E condition also applied a group format and intervention time was extended to match the staff-client contact time in the EP course groups. Thus, both the E and EP conditions might have contained active components that overall contributed to a lack of discrimination between both groups. Alternatively, exercise has been reported to have favorable effects on psychosocial outcomes such as self-efficacy, rumination, and mastery (Stathopoulou et al., 2006). It has been suggested that exercise may enhance a person's sense of mastery by creating a sense of independence and success through the command of a challenging pursuit (Craft \& Perna, 2004; Paluska \& Schwenk, 2000). Engaging in physical activity may also lead to distraction from negative emotions and unpleasant thoughts and may result in an improved affect following exercise sessions (Brosse et al., 2002; Hassmén et al., 2000). With the hypothesis that exercise leads to mental health benefits by directly affecting psychosocial outcomes (Stathopoulou et al., 2006), the unique contribution of the psychoeducation could have been too small to result in significant differences above those effected by the exercise.

Finally, we expected that women in the EP and E conditions would show greater improvements on outcomes than those in the WLC group. While there was a reduction in the number of negative self statements and rumination and an improved mastery within our treatment groups compared to the WLC, this 
decrease was not significant in the three-way interaction of outcome* group*time. As we did see some improvements on several psychosocial mechanisms in the intervention groups, it is possible that effects may emerge over time as participants grow more accustomed to using their skills. A comparison group that was tracked throughout the 12 month FU period would have allowed longitudinal data to detect whether increased improvement relative to a control group took place over time. For ethical reasons, we did not use a control condition across the whole study period. Because of this, our study design only allowed for the evaluation of the effects relative to a WLC group immediately after the intervention. After waiting an additional two months, WLC participants were free to enter the intervention and thus could not provide data to test long-term follow-up of intervention effects. To our best knowledge, other studies on the prevention of depression reported no long-term changes on variables such as self-esteem or self-efficacy in their control groups while the intervention conditions did improve over time (e.g. Allart-van Dam, Hosman, Hoogduin, \& Schaap, 2007; Groen, Pössel, AlWiswasi, \& Petermann, 2003). Similar patterns may have been present for the EWW course, but due to the study design it was not possible to detect these.

Some limitations of this study need to be addressed. First, an a priori power analysis that was performed to compare the three conditions on the primary outcome depressive symptoms estimated a sample size of 49 participants in each condition. The attrition of some participants before baseline measurements, as well as smaller than anticipated effect sizes on depressive symptoms, may have resulted in a type II error indicating that the present study did not have enough power to detect significant differences with small effect sizes. Secondly, we analyzed outcomes for several different psychosocial measures. The few significant outcomes that we have reported could be the results of an artifact related to the high number of tests we conducted. Thirdly, our analyses on changes in depressive symptoms and perceived stress indicated that the intervention was effective in certain subgroups of disadvantaged women. Since results on the primary outcomes suggest that the EWW does have benefits for women with lower depressive symptoms at baseline and for women with the lowest educational levels, a further exploration of moderating effects for the studied psychosocial variables is warranted.

Finally, our intervention outcomes on psychosocial variables did not show how these proximal constructs are related to stress and depressive symptoms and through which pathways and mechanisms mental health of low-SES women can be influenced. Many exercise intervention studies have measured 
depressive symptom outcomes, as these symptoms are an important component of mental health and quality of life (Conn, 2010). As described earlier, stress and depressive symptoms can be generally modeled as the last link in a chain of effects (Aneshensel, 2009), in which fewer and less adequate personal and social resources (e.g. rumination and lack of social support) are related to an increased risk for these mental health problems (McLeod \& Nonnemaker, 1999; Sterk, Theall, \& Elifson, 2006). At pre-test, the proximal outcomes included in this study were all significantly correlated with perceived stress and depression in the expected directions (e.g. women with more negative self statements had more depressive symptoms; $r=0.80$ ). While concurrent overall change between depression and the proposed mechanisms can provide some support for the mediating role for these mechanisms, an examination of the timeline of change can offer greater insight (Kazdin, 2001; Kazdin \& Nock, 2003). A longitudinal explorative study of the associations between the targeted psychosocial factors on the one hand and stress and depression on the other could give us more indications on which outcomes should be explicitly targeted in preventive interventions for low-SES women.

Several studies have shown that the Coping With Depression (CWD) course is effective in the prevention of new cases of major depressive disorders (Cuijpers, Munoz, Clarke, \& Lewinsohn, 2009). However, despite its widespread implementation among Dutch community mental health centers, low-SES groups profit less from this service (Smit, Bohlmeijer, \& Cuijpers, 2003). The cognitive orientation and written homework assignments included in the CWD training leads to frequent drop-out of participants from this group (Allart-van Dam \& Hosman, 2002). Furthermore, we do not know whether the CWD in its original format is able to elicit favorable effects among low-SES women. Studies that have evaluated the outcomes of the CWD course have not distinguished whether intervention effects on intermediate outcomes or depressive symptoms showed differential effects between socio-economic groups (Haringsma, Engels, Cuijpers, \& Spinhoven, 2005; Willemse, Smit, Cuijpers, \& Tiemens, 2004). Outcomes for the CWD that have been evaluated with disadvantaged populations give some indications that this approach could benefit low-SES women, but the populations in these studies are not comparable to low-SES Dutch women (Miranda et al., 2003; Muñoz et al., 1995).

The EWW intervention was developed to meet these concerns and has been tailored to the specific needs of low-SES women. A core element of the EWW course is its group based format in which the psycho-educative topics link up with body focused exercises. The main goal of the intervention is to reduce stress and depressive complaints among low-SES women (Lanen et al., 2008). 
In this study, the combined approach of the EWW intervention showed no additional effects for changes in psychosocial outcomes compared to women who only participated in exercise activities. Despite these outcomes, it would be too soon to conclude that a psycho-educative approach has no merit for lowSES women. A process evaluation of the EWW course (Van der Waerden et al., in press) indicated that women valued the EWW intervention and also indicated a subjective improvement on their mental wellbeing after participation. Also, as we did find some indications that participants in both intervention groups improved on outcomes such as negative self statements, rumination, and mastery, the EWW course can be still further improved to elicit larger changes in outcome variables. Knowledge on working mechanisms might further improve the design of effective interventions. In general, more knowledge is required on the minimal dosage of exercise needed to produce preventive effects in depression symptomatology (Dunn, Trivedi, Kampert, Clark, \& Chambliss, 2005). The same applies to the dosage needed for each of the themes in the psycho-educative part, including the use of homework assignments. However, we have developed an innovative intervention that is acceptable to and may have some beneficial outcomes for a disadvantaged group. In this light, this study on the effects of the EWW course represents an important next step in the area of prevention of depression for low-SES women. 
Chapter 8

General discussion 
146 Chapter 8 


\section{General discussion}

Mental health is fundamental to overall health and wellbeing. As a group at high risk for depression, women with a low socio-economic status (SES) from disadvantaged communities are the primary focus of this dissertation. While low-SES women may have a similar genetic and biological vulnerability to depression compared to other women, increased stress may account in part for the increased depression prevalence among women living in disadvantaged circumstances (Hauenstein, 1996; Hobfoll, Johnson, Ennis, \& Jackson, 2003). The Stress Process Model expands on the ways in which social structures come to influence disparities in mental health and indicates three different, but related pathways through which the stress process can contribute to these mental health disparities (Pearlin, 1989, 1999). First, disadvantaged social status decreases mental health because it exposes low-SES women to higher amounts of stress compared to the general population. Secondly, these women have limited access to the personal and social resources that may mediate and moderate stress. Stressors and chronic problems may, over time, erode the psychological and social resources that are available to low-SES women. Finally, low-SES women may be less successful in utilizing their social and personal resources. Together, these three pathways of increased psychological distress and diminished resilience may reduce the mental health of disadvantaged women and increase their risk of depression. Based on this theoretical framework and previous research that has documented the beneficial effects of psycho-education as well as exercise on depression, the Exercise without Worries (EWW) intervention was developed. The main goal of this intervention is to reduce stress and depressive complaints in low-SES women by increasing coping related competences and improving the balance between burden and capacity. The EWW prevention course has been tailored to the specific needs of this target group and combines psycho-educative topics with body focused exercises.

The central aim of this dissertation was to explore possibilities for the prevention of depressive symptoms in disadvantaged women, a population at high risk for mental health problems. For this purpose we addressed the broader context of prevention of depression in low-SES women and presented outcomes on the implementation and evaluation of a combined psychoeducation and exercise program targeted at this population. This chapter starts with an overview of the main results of the studies, followed by a general discussion. This discussion focuses on methodological reflections on the study design and provides recommendations to optimize the EWW course. Further, some theoretical reflections and implications of the outcomes for depression 
prevention in low-SES women and implications for future research are given. Lastly, this chapter presents a final conclusion.

\section{Main results}

The main findings of this thesis are briefly summarized below. A more extended review of these findings is presented in the respective chapters and the summary of this thesis.

The first part of this work addressed the broader context of prevention of depression in low-SES women. Chapter 2 focused on defining high-risk groups within this population segment. The outcomes of this study indicate that not all disadvantaged women necessarily have an equally high risk for mental health problems. In our population of low-SES women, perceived stress and individual-level SES factors were the strongest predictors of self-reported depressive symptoms. Multiple risk factors notably increased the chance of depressive symptoms in this group. Based on these findings, subpopulations among low-SES women could be targeted with preventive interventions according to their levels of risk.

There is a lack of systematic knowledge on the efficacy of preventive interventions to reduce depressive symptoms in low-SES women. Chapter 3 presented a systematic review and meta-analysis for the efficacy of preventive interventions to reduce depressive symptoms in low-SES women and of possible moderators to intervention effects. A number of promising programs have been developed specifically for low-SES women. On average these programs were found to reduce the level of depressive symptoms (estimated overall effect size of 0.31 ), with more than half of the studies showing medium to large effect sizes (range 0.00-1.14). We found no evidence that study sample characteristics, intervention characteristics or the research design of these studies moderated intervention effects.

Recruitment, willingness to participate, and retention in interventions are indispensable for successful prevention. The study in Chapter 4 investigated the effectiveness of different strategies for recruiting and retaining low-SES women in depression prevention, and explored which socio-demographic characteristics and risk factors within this specific target group are associated with their successful recruitment and retention. Although low-SES women are generally underrepresented in public health interventions, this study showed that it is possible to recruit and retain these women in a preventive intervention, to a larger extent even than the middle class population in the 
Netherlands. Especially recruitment through tailored strategies, such as referral for screening through the patient databases of GPs working in disadvantaged neighborhoods, proved to be an effective way to engage lowSES women.

The second part of this thesis reported outcomes of a process and effect evaluation of the EWW course. Chapter 5 described the theoretical background, program content, and process evaluation of the EWW course. Overall attendance rates were satisfactory, but $26 \%$ of the recruited participants did not actually take part in the course. Implementation fidelity was sufficient and, overall, participants reported being very satisfied with all organizational and content aspects of the intervention. The findings suggest that a nonstigmatizing approach to exercise and psycho-education fits with the preferences of low-SES women for preventive interventions.

In chapters 6 and 7, the efficacy of the EWW course was explored by means of a randomized controlled trial. One hundred sixty-one low-SES women with elevated stress or depressive symptom levels were randomly assigned to a combined exercise and psycho-educative (EP) intervention, exercise only (E) intervention or waiting list control (WLC) condition. Outcomes were measured before and immediately after the intervention and at 2, 6 and 12 month followups. In the overall target population of women from low-SES neighborhoods, no indications were found that the EWW course reduced depressive symptom and stress levels on the short or long term. We also assessed whether the EWW course directly influenced the psychological mechanisms that were targeted in the intervention. Overall, outcomes suggest that offering this preventive intervention to low-SES women did not produce any beneficial effects on important psychosocial resources associated with risk for depression in this group. We did find some indications that participants in the EP and E interventions improved on outcomes such as negative self-statements, rumination, and mastery, but these findings did not differ from the WLC condition at post-test. Given the lack of effects on psychosocial mechanisms targeted directly by the intervention, it is not surprising that we have not been able to find overall intervention effects on perceived stress or depressive symptoms. However, further exploration of the data indicated that for certain groups of disadvantaged women both exercise and combined exercise and psycho-education may be a viable prevention option to reduce mental health problems. Especially low-SES women with less severe initial problems or those with low educational attainment were found to benefit from participation in a preventive course. 


\section{Strengths and limitations of the study}

A major strength of this study is its specific focus on population at high risk, i.e. low-SES women. These women usually fly under the radar of health care professionals and researchers alike (Beeber et al., 2007; Gilliss et al., 2001; Miranda \& Green, 1999). While there are some challenges inherent to conducting research with high-risk populations in community-based settings, there has been growing interest in prevention studies with these groups (Le, Lara, \& Perry, 2008). The studies described in this dissertation used different methods to learn more about the prevention of depression and reduction of stress in disadvantaged women. We tested the efficacy of a preventive intervention by randomly assigning participants to intervention and comparison groups and monitoring specific predetermined outcomes. By doing this within the context of the community environment, we have improved the generalizability of the study, which enables us to maximize the public health significance of the study outcomes (Hohmann \& Shear, 2002). We have also been able to inform prevention experts and mental health care workers on how possible recruitment strategies and interventions can be tailored to the needs of individual women from low-SES populations. Different parties collaborated from the sectors of public health, mental health care, social and women's work, primary care and academia. Some methodological considerations and limitations of this study will be discussed in more detail below.

\section{Study population}

Despite the long research tradition on socio-economic status (SES) and social class, there is still ambiguity on the meaning of SES (Krieger, Williams, \& Moss, 1997). This was also the case for the present study. Our aim was to recruit women from disadvantaged neighborhoods in the EWW intervention. To further differentiate for SES status, we measured women's highest educational level to decide whether or not to include women from these urban neighborhoods in the study. Women were eligible when they had 10 years of formal education or less. However, over time we broadened these inclusion criteria to also admit women who, for instance, had a higher educational level, but who did not have paid employment or who had a lower than average family income. The screening showed that this group of women generally also had stress or depression scores above predetermined cut-off levels. This did make our study population more heterogeneous than initially intended.

In the Netherlands, educational level is a measure of adult SES that is frequently used to identify social inequalities in health (Regidor, 2006). 
However, even educational level can have different meanings for researchers from different countries. For many British authors, education is an indicator or pathway between early social circumstances and health (Regidor, 2006). This raises the question whether one of the other major measures of social class, occupation and income, should have been used as a primary criterion to select our target population. Occupation identifies technical aspects of work and is also associated with prestige, wealth, skills and specific working conditions. However, it is debatable whether occupation is useful to further differentiate between women living in disadvantaged neighborhoods. For instance, in our sample, only $28.2 \%$ of the women were in paid employment. The assessment of a person's economic resources, based mainly on measures of personal and household income but occasionally also on poverty or wealth, presents its own set of limitations in epidemiological and intervention research. Questions about income typically show a higher non-response rate than those about education and occupation, presumably because respondents are unwilling to disclose their financial situation (Muntaner et al., 2004). Thus, while our broadening of SES criteria has lead to a less homogeneous group of study participants, we feel that the current sample does reflect the different aspects of a disadvantaged social status in our society.

\section{Research design}

By comparing the EWW course to an exercise only module, we were able to investigate whether the combined course was more efficacious than a course that only focused on exercise. The study design did not include a psychoeducation only $(\mathrm{P})$ condition. This component has already been shown to be evidence-based in the reduction of depressive symptoms (e.g. Cuijpers, Munoz, Clarke, \& Lewinsohn, 2009; Cuijpers, Smit, \& van Straten, 2007). The Coping With Depression (CWD) course has 12 weekly sessions which focus on topics that are found to help prevent depression: social skills, skills in restructuring negative cognitions and behavioral activation to increase pleasant activities, training in increasing thoughts associated with positive mood and decreasing thoughts associated with negative mood, and elements from rational emotive therapy and cognitive therapy (Cuijpers et al., 2009; Lewinsohn \& Clarke, 1984). Despite the widespread implementation of CWD among Dutch community mental health centers, low-SES groups profit less from this service (Smit, Bohlmeijer, \& Cuijpers, 2003). The cognitive orientation and written homework assignments included in these training courses leads to frequent drop-out among this group (Allart-van Dam \& Hosman, 2002). We made several changes to the psycho-educative content for the EWW course. First of all, most of the topics were discussed less extensively than in the CWD 
course. Secondly, the psycho-educative contents and examples were adapted to the specific needs of low-SES women. Thirdly, the psycho-education was extended with other topics that have been found relevant for low-SES women, such as increasing feelings of self-respect, self-esteem, and mastery, and that help build boundaries to reduce stressful situations. Finally, the psychoeducation was coordinated with the exercise component in the course with the aim of reciprocally reinforcing the message. Because of these adaptations, our intervention is not completely comparable to the CWD psycho-education, which possibly explains the differential effects for the EWW course. Moreover, research has not yet fully determined what the active ingredients of the CWD course are and whether it is necessary to cover all modules (Cuijpers et al., 2009). Alternative versions of the course, such as CWD for older adults and migrants, have also been adapted by reducing the number of sessions and simplifying the texts used in the intervention. There is no indication that these versions are less effective than the full versions for adults (Haringsma, Engels, Cuijpers, \& Spinhoven, 2005; Miranda et al., 2003). This study showed no significant additional value of combining psycho-education with exercise training. However, this does not mean that psycho-education by itself does not have beneficial effects for low-SES women. Women in the EP group valued the intervention they received and indicated a subjective improvement on their mental wellbeing after participation. Also, as we did find some indications that participants in both intervention groups improved on outcomes such as negative self-statements, rumination, and mastery. These outcomes should be explored in more depth to better understand how the EWW course can be further improved to elicit larger changes in outcome variables.

The lack of any additional effect for exercise combined with psychoeducation could lie in the nature of the exercise only (E) condition. In the present trial, this condition was conducted in a group based format as was the EP condition. While the $\mathrm{E}$ group did not receive the psycho-educative component, the duration of the meetings did match the staff-client contact time in the EP course groups. This extended time could have provided more opportunities for social interaction and individual support, which in turn might have resulted in improved mental health outcomes and thus contributed to the lack of differential effects between the E and EP conditions. However, neither group showed improvements in the supportive interactions from their social support network. The E and EP conditions may both have contained active components which overall contributed to a lack of discrimination between the groups.

For ethical reasons, we did not use a control condition across the whole study period. We included a waiting list control group for the evaluation of the 
effects immediately after the intervention and at 2 months follow-up. Thus, the WLC group was followed for four months before they began the EWW course. During this period, the perceived stress and depressive symptom scores in the WLC group also decreased, as well as most of the outcomes that were targeted in the intervention. This reduction among WLC participants may reflect regression to the mean as improvements can occur in a population that already has increased depressive symptoms and stress levels at the time of enrollment in a study. However, we did not monitor whether participants in the WLC group sought additional preventive activities or professional help during their waiting period. And for this reason, we cannot exclude the effects of other activities on their improved short-term outcomes. After the four month waiting period, WLC participants were able to enter the intervention and, thus, could not provide data for the long-term follow-up of intervention effects. The absence of a control group at all follow-ups limits our interpretation of possible long-term intervention effects. Also, a long-term follow-up of the WLC group could have indicated whether or not their initial reduction in symptoms continued over time.

\section{Measures and measurement setting}

This study did not use a diagnostic instrument to assess the presence of clinical depression at pre-test, post-test and follow-up, but used depression self-reports only. Cut-off scores on self-report questionnaires for screening purposes were chosen to reflect a balance between sensitivity, specificity, and positive predictive value. Nevertheless, the depressive symptomatology of high scorers does not necessarily meet the criteria of a depressive disorder as determined by a clinical interview (Santor, Zuroff, Ramsay, Cervantes, \& Palacios, 1995). This is also pertinent to low-SES women (Thomas, Jones, Scarinci, Mehan, \& Brantley, 2001). There are a number of factors that support the use of selfreport measures for selecting participants in depression prevention trials. First, inclusion of participants in an intervention based on the outcomes of a depression screening instrument reflects current prevention practice. This supports the external validity of our research, since it did use selection criteria for participants at high risk for depression that are also applied in real-life prevention settings. Secondly, when using self-report measures to select participants, only a small proportion of the total study sample might actually qualify for a depressive disorder. Thirdly, our meta-analysis on psychosocial interventions for low-SES women included the use of a diagnostic measure in the moderator analysis. Outcomes did not show significant differences in effect size between studies that excluded depressive disorders at baseline and 
those that did not assess the potential presence of this disorder. Finally, the prevention of depressive symptoms or the reduction of the intensity of these symptoms is also relevant for preventing depression incidence (Clarke et al., 2001; Willemse, Smit, Cuijpers, \& Tiemens, 2004).

The results relied on self-report questionnaires that, because of their length and level of difficulty, could have posed problems to a low-SES population. We tried to accommodate response by having a trained interviewer to administer the questionnaires. All measurements were conducted face-to-face with the same interviewer, except the 2 month follow-up which was conducted by phone. This could have led to non-specific intervention effects, with participants, for instance, giving socially desirable responses. The finding that study participation, either as a control or experimental participant, could lead to improvement in women's mental health was also found in previous research with low-SES women (Carpenter, 1998; Peden et al., 2000). Low-SES women often feel isolated and have a low sense of belonging (Choenarom, Williams, \& Hagerty, 2005; Green \& Rodgers, 2001). Regular attention from the interviewer could possibly have led to a Hawthorne effect (Olson, Verley, Santos, \& Salas, 2004).

A final limitation concerns implementation fidelity, which was measured by questionnaires completed by the trainers after each session. This could have caused some expectation bias related to implementation, which means that observers may have provided data toward the expected outcome. The reliability of implementation fidelity would have been stronger if a representative sample of the course sessions could have been directly recorded and coded for deviations from the manual by an independent observer. However, it is possible that the presence of a camera or non-participating observer would have lead to reluctance among participants to share their - often personal - experiences or to feel they could exercise freely.

\section{Participant attrition}

Another final limitation of the study was attrition. Of the 161 women enrolled, 12 dropped out before baseline measurement and another 19\% (28 women) discontinued the study before completing their final follow-up measurement. Given the often competing demands for time and resources among low-SES women (Beeber et al., 2007; Weinreb, Perloff, Goldberg, Lessard, \& Hosmer, 2006), this level of attrition was not surprising. Reasons for drop-out included losing interest in the study, moving out of the region or the research team no longer being able to contact the participant. Combined with a larger effect size $(d=0.40)$ used in our a priori power calculation than those we found in our 
study population, the present study may not have had enough power to detect significant differences.

\section{Recommendations to optimize the Exercise without Worries intervention}

With the current emphasis on evidence-based interventions, there might be reason to conclude that a program such as the EWW intervention should be stopped because of its limited effects. However, despite the absence of additional effects for its combined exercise and psycho-education approach, the EWW course can possibly contribute to the prevention of stress and depressive symptoms among low-SES women more than it did in this study. In their paper on randomized preventive trials in mental health, Brown and Liao (1999) examined four reasons why well designed trials sometimes fail to demonstrate the desired effect: i) The respondents who are recruited and who take part in the intervention are not the ones who stand to benefit most, ii) the intervention itself is not efficacious; that is, it is not beneficial under optimal conditions; iii) the level of program implementation is not sufficient to produce measurable results; and iv) participation among those who are enrolled is limited. We will consider the outcomes of the EWW intervention in the light of these four reasons, along with recommendations for improvement of this intervention.

\section{Improving target group selection and recruitment}

The outcomes of the effect evaluation suggest the possibility that the women who were recruited and took part in the EWW intervention are not the ones who might benefit most. Chapter 2 indicated that not all disadvantaged women are at equal risk for depression, and especially those with an accumulation of socio-economic risk factors could benefit from preventive action. Furthermore, we found that women eligible for the EWW course were more likely to assent to an intake when they experienced higher stress and depressive symptom levels (Chapter 4). However, outcomes of the effect evaluation suggest that the EWW course in its current lay-out may be a viable prevention option especially for those low-SES women with less severe initial problems (Chapter 6).

Selection of the preventive target group can be improved through a stepped approach. First, women with an accumulation of socio-economic risk factors for depressive symptoms should be the focus of targeted screening. The EWW 
course could be offered to women who have elevated symptoms, but whose scores are still below cut-off scores on self-report questionnaires, reflecting high or even clinical levels of symptoms. Women with more severe complaints could be guided to other prevention or treatment options that better suit their needs.

The recruitment of disadvantaged women with an accumulated risk but low current symptoms warrants special attention. This group may feel that their functioning and mental health are not heavily affected, and thus that they do not particularly need to participate in a prevention program (Arean \& Miranda, 1996; Ireys, DeVet, \& Chernoff, 2001). It has been suggested that local community services and media campaigns might be used to reach these subjects and to offer them educational messages about depression and the importance of seeking help earlier for this condition (Ayuso-Mateos et al., 2007). In general, service providers may need to be more active in engaging potential participants from disadvantaged groups. The daily hassles and often chronic pressures on low-SES women frequently lead to competitive priorities that take precedence over keeping appointments for an intake. For example, some women had to cancel intake appointments because of meetings they were required to attend for a debt reform program that they were participating in. Although these women may have wanted to keep the intake appointment, their debt reform program took priority over an intake for the voluntary EWW course. Recruitment of low-SES women should involve repeated efforts with prospective participants to schedule appointments and engage them in the intervention (Azocar, Miranda, \& Dwyer, 1996; Miranda \& Green, 1999).

\section{Improving intervention content}

When an intervention has been developed with a lack of theoretical and empirical support for its components, it is possible that the intervention is not efficacious, that is, there will be no beneficial effects under optimal conditions. Nevertheless, this was not the case for the EWW intervention. The topics discussed in the psycho-educational component were all related to risk factors for depression in low-SES women as described in the Stress Process Model of mental health disparities (Pearlin, 1989, 1999; Pearlin, Menaghan, Lieberman, \& Mullan, 1981). These topics were further extended with other evidence-based risk factors for depression (Cuijpers et al., 2009; Lewinsohn \& Clarke, 1984). The exercise component of the course was grounded on previous research that indicated that exercise may have both a preventive and a therapeutic impact on mental health problems and stress and that has been found to be effective in reducing depressive symptoms (Alderman, Rogers, Johnson, \& Landers, 2003; Stathopoulou, Powers, Berry, Smits, \& Otto, 2006). Yet, despite this theoretical 
foundation, only minor effects were found on psychosocial outcomes, perceived stress and depressive symptoms.

\section{Improving the psycho-education}

The Stress Process Model suggests that to reduce the incidence of mental health problems in disadvantaged women, prevention and intervention strategies can focus on increasing women's access to and effective use of psychosocial resources, or reducing their exposure to stress. The EWW course targeted these psychosocial resources by influencing negative thought patterns, rumination, self-esteem, self-efficacy, mastery and social support of the women participating. So far, outcomes from this study have indicated that the EWW course was not able to increase participants' access to and effective use of these psychosocial resources, which possibly explains the lack of overall effects on stress and depressive symptoms.

An increased focus on the second strategy, reducing the exposure to stress, should be further explored. Theory suggests that stressors and stress proliferation have an important contribution to depressive symptoms among low-SES women. It has further been observed that low-SES women characteristically use coping mechanisms that exacerbate, rather than reduce, stress (Banyard \& Graham-Bermann, 1998; Turner \& Lloyd, 1999). The important role of perceived stress for the prediction of depressive symptoms among low-SES women from urban neighborhoods was also illustrated in Chapter 2. The outcomes of the process evaluation (Chapter 5) indicate that coping with stress and learning to relax were important reasons for participation in the EWW course. In its current form, the EWW course does not directly target low-SES women's experience or generation of stress or methods that these women could use to reduce stress. The only intervention element that is directly tied to stress reduction is the relaxation exercise at the end of each EWW lesson (Salmon, 2001; Steptoe, Kimbell, \& Basford, 1998).

Our meta-analysis on psychosocial interventions for low-SES women gave no indications that programs including stress management techniques are less effective than those focusing on interpersonal therapy or social support techniques. However, there are few interventions to our knowledge with a specific focus on stress management that specifically target low-SES women. The content of available programs might give some leads on how stress and stress management can occupy a more central role in the EWW course. In the 1980s, Tableman (Tableman, 1987, 1989) proposed a multidimensional stress management training program for low-income women. This specific program provided participants with information on life coping skills, goal setting to manage the impact of life changes, problem solving and decision making skills 
and specific stress management skills to reduce stress generated by unplanned or uncontrollable events. Further, Hauenstein $(1996,1997)$ developed the WAIT program, a stress inoculation training to reduce physical and psychological responses to stress, and enhance resilience among disadvantaged rural women with depression. This program focuses among other issues on basic knowledge and understanding of stressors, the stress response, and depression risk. As a second step in the program, women develop specific skills to identify common stressors in their lives and in their usual stress responses. The final steps of the program focus on acquiring self-care and problem solving skills in response to stress. Thus, a hands-on focus on problem solving and stress management skills appears to represent a viable option for expanding the intervention. We suggest maintaining the relaxation exercises, since the process evaluation of EWW indicated that of all intervention topics, participant appreciation was highest for the relaxation exercises. Relaxation exercises may well be incorporated in a stress management approach.

\section{Improving the exercise component}

An optimal dose in terms of exercise frequency and duration needed for treatment and prevention efficacy has not yet been fully defined (Faulkner, 2009). However, there are some indications that physical activity in the amount recommended by public health norms (150 minutes per week) results in rates of response and remission comparable to those reported in trials of cognitive behavioral therapy and antidepressant medication (Anderson et al., 1999; Brown, Ford, Burton, Marshall, \& Dobson, 2005; Dunn, Trivedi, Kampert, Clark, \& Chambliss, 2005). The exercise component of the EWW involved stretching, muscle reinforcement, flexibility, body focused exercise, and relaxation. This was done at a low to moderate intensity for 60 minutes so that the physical exercise was not too strenuous compared to participants' habitual exercise levels. Generally, exercise is said to reduce depressive symptoms because of physiological, psychological and social support mechanisms (Paluska \& Schwenk, 2000; Stathopoulou et al., 2006). While its low exercise intensity makes it unlikely that the EWW intervention activates any physiological mechanisms associated with the reduction of depressive symptoms, we assumed that the course would be able to stimulate psychological and social support mechanisms (Brosse, Sheets, Lett, \& Blumenthal, 2002; Salmon, 2001; White, Kendrick, \& Yardley, 2009). There have been indications from previous intervention studies that the best psychological benefits are probably derived using moderate exercise intensity as opposed to a very low or very high intensity (Goodwin, 2003; Teychnne, Ball, \& Salmon, 2008). Despite the favorable effects that have been reported for the reduction of 
depressive symptoms with exercise below the public health norms (60 to 150 minutes per week; Anderson et al., 1999; Brown et al., 2005), the EWW course did not result in improved outcomes. Yet, before increasing the intensity of the EWW intervention, we need more knowledge on the minimal dosage of exercise needed to produce preventive effects in depression symptomatology (Dunn et al., 2005). Since low-SES women often do not have intensive exercise habits (Ball, Salmon, \& Crawford, 2006; Droomers, Schrijvers, Van de Mheen, \& Mackenbach, 1998), making the exercise component too strenuous might lead to increased attrition among this target group. An option may be to gradually increase the exercise intensity over the duration of the course.

To further attain the public health norm for physical activity, women can be encouraged to adopt and maintain regular physical activity in or around their homes or in other community settings. Small group instructional approaches for promoting physical activity in low-SES women have been found effective in increasing women's readiness for physical activity (Albright et al., 2005; Collins, Lee, Albright, \& King, 2004). As part of the EWW course, group members can be stimulated to go walking together once a week. Previous studies on the adoption of a regular walking routine among low-SES women indicated that being part of a group with a consistent membership and meeting time encouraged women to participate in a weekly group walk (Perry, Rosenfeld, \& Kendall, 2007). However, as unfavorable perceptions of the neighborhood (e.g. busy traffic conditions, not suitable for walking) have been shown to influence physical activity among disadvantaged populations (GilesCorti \& Donovan, 2002; Kamphuis et al., 2009), improving infrastructural services to make the environment more attractive could be necessary.

\section{Improving program duration}

A lack of intervention effects can also be attributed to a level of program implementation that is possibly insufficient to produce measurable results (Brown \& Liao, 1999). The absence of clear outcomes could indicate that the 8week EWW course was too short to exert the full psychological benefits for its target group of low-SES women. A meta-analysis has indicated that depression prevention interventions with eight sessions or more are more effective than shorter interventions (Jané- Llopis, Hosman, Jenkins, \& Anderson, 2003). The CWD, as well as most other prevention of depression programs showing evidence of efficacy, have 12 to 15 sessions (e.g. Clarke et al. 2001, Cuijpers and Dekker 2005, Muñoz et al. 2007). Compared to these interventions, the EWW course included more topics in fewer sessions. The variety of the topics may have been too broad for the short time frame of the course, which perhaps made it difficult for the participants to internalize the subject matter. As a 
result, participants' knowledge of intervention themes may have remained too limited to effectively counter the structural stressors and problems present in their daily lives. The process evaluation gave some indications that this played a role. Women indicated that they thought the course was too short, with some of them expressing concern about whether they would be able to sustain and implement what they had learned in their daily lives after only 8 or fewer sessions.

A minimal intervention duration may also be necessary for the beneficial properties of exercise to take effect. The few studies that have investigated the influence of frequency and duration of exercise on depressive symptoms have given some evidence for a dose-response effect of exercise on depression (Goodwin, 2003; Hassmén, Koivula, \& Uutela, 2000). Depression can be reduced by 10 to 12 weeks of exercise (Dunn et al., 2005; Ströhle, 2009). Based on these considerations we suggest increasing the intervention from 12 to 15 sessions.

\section{Improving participation}

A fourth reason why the EWW intervention may not have succeeded as intended is that the participation among women who were enrolled was limited. Previous research has indicated that low-SES groups drop out more frequently from psycho-educative interventions due to the cognitive orientation and written homework assignments included in these courses (Allart-van Dam \& Hosman, 2002). The design of the EWW intervention takes these possible content barriers for mental health service use among low-SES women into consideration with its combined and adapted psycho-education and exercise focus. Furthermore, possible instrumental barriers to participation were also removed in this course (Azocar et al., 1996; Miranda et al., 2006). The EWW course was offered free of charge, and additional expenses related to childcare and public transport were reimbursed. To further facilitate attendance, participants were called and reminded of the course shortly before it started, and those who missed a session without giving prior notification were also contacted. This was in line with recommendations of previous studies with low-SES women that have shown that telephone prompting, letters, and consultations are effective means of increasing attendance rates (Miranda \& Green, 1999; Azocar, Miranda, \& Dwyer, 1996). Despite these strategies, attendance at the intervention meetings was lower than expected. Of the eligible participants who consented to take part in the EWW course, almost $30 \%$ did not attend any of the course sessions. One explanation might be that low-SES women are often uncomfortable saying no and will thus passively assent, resulting in non-attendance (Le et al., 2008). Nevertheless, this rate does 
not compare unfavorably with other group preventive interventions for lowSES women reported in other studies. Non-attendance and attrition rates among this population have been reported to range anywhere from $12 \%$ to $83 \%$ (Zlotnick et al., 2001; Ayuso-Mateos et al., 2007, Miranda et al., 2003).

On average, women who did start the EWW course attended five out of eight EWW sessions. While some reported explanations for non-attendance were related to illness or family circumstances, it is possible that psychological barriers prohibited participation in the intervention (Grote, Zuckoff, Swartz, Bledsoe, \& Geibel, 2007). People with low incomes are more likely than those from the middle classes to express concerns about being stigmatized by community members, employers, health care professionals, and family members if they get help for mental health problems (Alvidrez, 1999). Including the exercise component in the EWW intervention may have reduced stigma concerns associated with mental health care; for instance, women can refer to the intervention as an exercise class. While we did not find that insufficient participation further decreased the intervention's effect, it should still be a point of attention for future implementation of the course. Instable attendance rates may disturb the cohesion and trust within the group, and therefore potentially affect all group members. One possible way to address and overcome potential instrumental or psychological barriers within the setting of the EWW course is to use the intake to explain the objectives of the course and record background information, as well as elicit and resolve women's ambivalence about participation in the intervention or any instrumental barriers. To this effect, Grote et al. (2007) developed a pretreatment engagement intervention which uses both ethnographic interviewing and motivational interviewing techniques to resolve instrumental and psychological barriers to mental health care. The ultimate goal of this engagement interview is to help potential participants make a commitment to the intervention. 


\section{Reducing depression in low-SES women: the limitations of a downstream approach}

A well-known prevention parable by Zola (1986) relates to well-intending prevention staff working to save people from drowning in a river, instead of going upstream to find out why those people are in the river ${ }^{1}$. This metaphor can be extended to the prevailing approach of reducing depression in low-SES women. To achieve beneficial effects for the mental health of low-SES women, and ultimately reduce mental health disparities, interventions generally aim to enhance psychosocial resources among participants or teach their target group how to effectively mobilize these resources. Several interventions with these aims have been implemented, and some have contributed to improving the mental health of their participants. However, this "downstream" approach to reducing depression (or mental health problems in general) among low-SES women has not yet led to large successes. The mental health status of low-SES women as a group is still worse than that of the general population (De Graaf, Ten Have, \& Van Dorsselaer, 2010; Van den Berg \& Schoemaker, 2010). Moreover, these mental health inequalities have not decreased over the past decade (Dalstra, Kunst, Geurts, Frenken, \& Mackenbach, 2002; Mol et al., 2005).

Link and Phelan $(1995 ; 2010)$ have proposed some indications on why interventions targeting the proximal determinants of health, such as psychosocial resources, have proved ineffective in eliminating health inequalities. They suggest that the identification of intervening or mediating variables divert attention from the social conditions that put people at risk for general health problems in the first place (Link \& Phelan, 1995). There are several limitations within the downstream approach to depression prevention in disadvantaged women. The first limitation is related to the overall limited efficacy of the current interventions that target individual risk and protective factors for depression among this population. Meta-analytic evidence of intervention effectiveness (Chapter 3) and the outcomes of the EWW intervention show that these interventions do not necessarily result in a large decrease in depressive symptoms. Although the effect sizes are consistent with those found in earlier meta-analyses for depression prevention and mental

\footnotetext{
1 "Suppose you are standing next to a river, and you see someone drowning as she floats downstream. You jump into the river and pull her ashore. As soon as you've done that, you see another person in trouble, again floating downstream, and you rescue him as well. Every time you've saved one person, you see another, and another. After you've dragged another drowning body out of the river, you're thoroughly exhausted and you know you don't have the energy to save one more person, so instead you decide you must go upstream to find out what is causing these people to end up in the river."
} 
health promotion (Jané Llopis et al., 2003; Wilson and Lipsey, 2001), they remain only modest.

A second limitation to downstream approach is related to the selection of populations to target with preventive action. Outcomes from previous research, as well as our study presented in Chapter 2, indicate that a targeted high-risk approach is viable even among low-SES women, a group which is generally considered to be a population at high risk for depression. When multiple risk factors are present in the life of a woman, her chance of depressive symptoms notably increases. While women with more depressive symptoms were more motivated to participate in preventive activities and were better retained in the course (Chapter 4), there are also some, perhaps even paradoxical, indications that this group benefits least from the preventive activities included in this study (Chapter 6). Also, the public mental health impact is barely visible since this targeted high-risk approach reaches only a small portion of this population.

A final - but perhaps the most substantial - shortcoming of our current preventive approach for low-SES women is that it leaves the social inequalities that generate their disproportionate exposure to stress and inadequate psychosocial resources intact. Our interventions now target women, or groups of women, based on their risk status and aim to empower them to counter the negative influence of the social setting in which they live. While recruited women may benefit for a while from their participation in a preventive intervention, it is highly conceivable that over time their newly acquired psychosocial skills or support will erode in the face of ongoing adversity. Even if a woman is capable of sustaining the skills she has been taught and remains in good mental health, there will always be new women at risk and in need of the benefits of programmatic intervention.

\section{Going upstream: a multi-faceted prevention approach}

To really reduce mental health disparities between populations, another approach is needed that looks upstream at the social inequities that generate higher depression prevalence among disadvantaged women. To alleviate structural stressors, combinations of intervention themes and methods in multiple domains of women's lives could further increase mental health outcomes among low-SES groups. A feasible method to reduce depression among low-SES women is a multi-faceted approach that combines targeting high-risk women with population-based measures. The concepts of high-risk strategies versus population-based measures for prevention originate from the 
ideas proposed over two decades ago by Rose (1985). In brief, high-risk prevention targets individuals identified (e.g. through screening) as having elevated risk for a certain adverse health outcome. The population strategy, however, targets a whole population with prevention activities regardless of variation in individuals' risk status (McLaren, McIntyre, \& Kirkpatrick, 2010; Rose, 1985, 1992).

The perspectives of Rose fit well into the tradition of social epidemiology and health promotion and within the concept of social structures that eventually lead to mental health disparities as expanded in the Stress Process Model, which has also been incorporated in the context of the current research. In order to decrease low-SES women's risk of depression, and more in particular their mental health disparity with other populations, macro-level interventions and policies that target structural inequalities (school attendance, basic welfare and care services, poverty, unemployment, neighborhood disadvantage, race/ethnicity, social cohesion) should be implemented. In the words of Link and Phelan (1995; 2010), to reduce (mental) health inequalities, we need to target the structural conditions that put people "at risk of risks". This call to a more upstream, or policy-oriented approach to prevention, is not new and has to a certain extent been underscored by policy makers and prevention advocates alike (Albee, 1986, 1996; Kickbusch, McCann, \& Sherbon, 2008; Kickbush, 2009; Reid, 2004). Realistically, however, large-scale social policy changes such as taxation, housing policies, or alleviation of low incomes are difficult to enact for cultural and political reasons (Thoits, 2010). Effects will often only be visible in the long term. Moreover, while the benefits of an upstream or population-based approach may in the long run outweigh their costs, the absolute cost of intervention is nevertheless likely to be high (Link \& Phelan, 1995; Post, Zwakhals, \& Polder, 2010).

More manageable and politically feasible methods within the upstream approach might target the meso-level structures in which disadvantaged women live and work: their neighborhoods or communities (Aneshensel, 2010; Mair et al., 2008; Matheson et al., 2006). From the perspective of the Stress Process Model, the mental health impact of a neighborhood can possibly be mediated by, for instance, increased stress through neighborhood deprivation (Latkin \& Curry, 2003; Matheson et al., 2006; Silver, Mulvey, \& Swanson, 2002) and reduced social cohesion within neighborhoods (Mair, Diez Roux, \& Galea, 2008; Mulvaney \& Kendrick, 2005). Neighborhood deprivation may also influence other areas of people lives such as work and personal relationships (Aneshensel, 2009). In essence, interventions focusing on disadvantaged neighborhoods target the mediating physical and social infrastructures, rather than macro-level social inequalities (Thoits, 2010). Since altering the physical 
surroundings in which people live has the potential to reach everyone within those surroundings, these interventions are able to reach large numbers of people as intended by population-based approaches. In recent years, more initiatives have been taken to this effect in the Netherlands. In 2007, the Dutch government made the decision to spend several billion euros to turn 40 'problem' districts into 'power' districts (Regeringsverklaring, 2007). The aim of this ' 40 districts' plan is to increase the livability of the districts, focusing on problems of housing, employment, education, integration and safety. The emphasis on 'power districts' and financial support from both national and local governments to improve them may contribute to the mental wellbeing of low-SES women.

However, the Stress Process Model still indicates the possibilities for the reduction of mental health disparities through the individual level. Besides population-based approaches, we will still be in need of "downstream" interventions (e.g. the EWW course, psychosocial interventions, stress management courses, individual empowerment) for those women who have already ended up "downstream in the river". There is increasing evidence that a multi-component intervention approach targeting both the individual and the community could bolster outcome effectiveness (Greenberg, Domitrovich, \& Bumbarger, 2001; Jané Llopis, Hosman, Jenkins, \& Anderson, 2003; Nation et al., 2003).

\section{Recommendations for research and practice}

The proposed multi-faceted approach to depression prevention in low-SES women, further enhanced by the specific knowledge gained by the study on the EWW intervention, has implications for both prevention researchers and practitioners focusing on depression among disadvantaged women.

\section{Recommendations for research}

In this study, we have aimed to identify which low-SES women are most at risk for depressive symptoms and give indications of a possible risk profile. The risk profile found in this research cannot be automatically applied to other populations, like the low-SES populations in large cities. This especially applies to cities and districts with large ethnic minority groups since these were relatively underrepresented in the overall population of Maastricht and thus in our sample. The risk profile for a more ethnically diverse population could be different from the profile presented in this study. We suggest, therefore, a 
replication of these methods among other low-SES communities that include more ethnic minority women.

Furthermore, in the Netherlands only four interventions for the prevention of depression have been specifically developed for and targeted at people with low SES (Busch \& Schrijvers, 2010). The EWW intervention has been the first to be rigorously tested on its effectiveness. The outcomes suggest that this intervention, although it has been developed for low-SES women in general, is more successful in specific subgroups of participants. The same might well apply to the other available interventions for low-SES populations. Future prevention research should pay attention to increasing knowledge about moderators, as it informs both research and practice about the possible limits of interventions and about who benefits the most.

Related to this is the need for more information on which factors most effectively buffer the effects of stress and improve the mental health of low-SES women. If we are able to identify these factors we could use this knowledge to enhance preventive intervention content and disseminate these programs for wider use in low-SES communities. This knowledge can be drawn from primary research about moderating factors. However, it should also be taken into account that purely research-driven knowledge on these buffering factors might lead to the development of interventions that do not necessarily appeal to low-SES women. Therefore, any such research should be complemented with knowledge on which life stressors and mental health goals are important for the women themselves. Understanding women's social cultural experiences and what they consider important approaches to ameliorate stressors in their lives could benefit both intervention development and implementation.

This research has shown that a depression prevention intervention that uses exercise as an intervention component is an attractive alternative preventive method for low-SES women. However, our knowledge on the minimal dosage of exercise needed to produce preventive effects in depression symptomatology is still limited (Dunn et al., 2005). Moreover, we do not know yet through which mechanisms exercise can benefit mental health. Exercise as a means to prevent depression has become an increasingly popular intervention method in the Netherlands. In the last couple of years, several exercise interventions have been developed and implemented (e.g. Liever Bewegen dan Moe, Beweeg uit je Dip, Fit uit je Dip). However, before policymakers move to a more sustained implementation of these programs, we need to find out what the potential mediators are and how these can benefit the effective prevention of depression. In 2010 the national taskforce Exercise and Depression was initiated. Participants are research and practice organizations such as the Netherlands Institute for Sport and Physical Activity (NISB), the Netherlands 
Institute for Mental Health and Addiction (Trimbos Institute), Maastricht University, and several mental health care organizations. One of the principal aims of this taskforce is the surveying and combining of knowledge on nationally developed interventions for exercise and depression prevention. The long-term goal is the development of a protocolized and combined life-style intervention for people at increased risk for depression. To reach this latter goal, currently developed exercise for depression interventions should be more rigorously evaluated. Most of the currently implemented interventions have not even been tested by simple pre-post designs.

\section{Recommendations for practice}

Our results indicated that low-SES women with an accumulation of psychological and socio-economic risk factors were most at risk for depressive symptoms. Future depression prevention programs among low-SES women should not consider this group as a homogeneous population, but specifically target women who face an accumulation of socio-demographic risk factors. These are likely the women who will benefit most from participation in a preventive intervention. We suggest that providers who have frequent contact with women from disadvantaged communities be more aware of those women at accumulative risk for depressive symptoms. To identify those who might be in need for preventive intervention, more targeted screening efforts of women with accumulated risk are needed. While primary care practitioners or mental health professionals form trusted sources in the community that can identify high-risk women, our study has indicated that many low-SES women are still overlooked. Screening of women in GP databases indicated that approximately $49 \%$ of low-SES women residing in disadvantaged neighborhoods had stress or depressive symptoms above cut-off levels. While most general practitioners see diagnostic tasks for psychological or social problems as part of their work description, systematic attention to the early detection of stress or depression is still limited (Laurant, 2001; Noordman, 2007). Also, when community-based service providers were asked to directly refer women with these symptoms to the EWW course, this occurred infrequently. Although it seems that general practitioners prefer to invest in close collaboration with mental health workers, rather than to invest in providing mental health care themselves, referral to mental health care is not always optimal (Cox, Van Marwijk, \& Boer, 2006; Emmen, Meijer, Heideman, Laurant, \& Verhaak, 2007).

While it is true that primary health care is a setting that is regularly visited by disadvantaged women (Ten Have, Oldehinkel, Vollebergh, \& Ormel, 2003; Ten Have, Vollebergh, Bijl, \& De Graaf, 2001), low-SES women often attend other professionals or social service agencies as well. Increased collaboration 
between different partners in primary care, public health and social service settings is necessary to provide better care for these women. Psychiatric nurses located in primary health care can facilitate this collaboration within a local setting. While low-SES women are willing to participate in preventive action, outreaching recruitment methods are necessary to make them aware of the available services and to engage them. A primary requisite is that mental health nurses in primary care are aware of the services that other collaborators offer for this group so that they can refer women to appropriate care and welfare structures based on their screening outcomes. Overall, a workable solution might be 'stepped' referral and care. Most demographic or socioeconomic risk factors included in the accumulated risk score can be identified by asking a few simple questions (e.g. on financial situation and marital status), which requires no extra investment in time or money. Since perceived stress levels seem to be the best predictor of depression, their presence could be determined as well, which can be relatively easily done, for instance, by the mental health nurses. Overall, it is important to note that these efforts are embedded in an appropriate care structure so that women are not only screened for depression, but also receive suitable subsequent care. Particularly if a large range of tailored preventive interventions are offered to women, their willingness to participate in preventive interventions might increase. Whatever the type of intervention that is offered to low-SES women, it is important to pay more active attention to their retention from the first contact onwards.

Outcomes for the present EWW course show that this intervention is most suitable for those women with elevated, but still low to moderate, symptom scores. When selecting women for participation in the EWW course, this might suggest that those women with higher levels of depressive symptoms might be served better by a more intensive alternative treatment or by prolonged contact after the end of the course, such as integrated in a stepped care model. The possibilities for improving the contents of the EWW course should be further explored in consultation with public health, mental health care and welfare services. Also, qualitative approaches can help to incorporate low-SES women's perspectives of what provokes stress in their lives. Qualitative methods, such as focus groups or ethnographic interviews, can give more insight into the meanings that women ascribe to their experiences - how they make sense of the world (Hohmann \& Shear, 2002; Ware, Tugenberg, Dickey, \& McHorney, 1999). This insider point of view could shed more light how the EWW course may contribute to the mental wellbeing of future participants.

The previous practice recommendations are, however, all still centered on the provision of individual-level prevention while population-based approaches might lead to increased mental wellbeing for all. For low-SES 
women to be able to benefit from this individual approach, the necessary conditions for them to do so should be in place. To really contribute to the reduction of depression incidence among low-SES women, targeted prevention courses need to be concerted with meso- and macro-level interventions and policies that target structural inequalities.

\section{Conclusions}

Based on the research presented in this dissertation, we can draw some general conclusions on the prevention of depression in low-SES women. First of all, not all disadvantaged women are at equal risk for depression and especially those with an accumulation of socio-economic risk factors, but low symptom scores, could benefit from preventive action. Contrary to popular belief, it is possible to recruit low-SES women at risk for participation in depression prevention, although this requires an outreach approach and particular attention to the elimination of barriers to their participation and retention. While some psychosocial interventions are specifically designed for the reduction of depression in low-SES women, their overall effectiveness should be improved. Also for the EWW course, several options to improve its effectiveness were noted. While this intervention is acceptable for disadvantaged women, its overall effects on psychosocial outcomes, stress and depressive symptoms are short term and are limited to certain groups of participants. It is likely that the adversities that low-SES women encounter in their daily lives slowly erode the preventive effects that can be achieved from participating in indicated prevention efforts. Thus, the current focus on targeted individual prevention should be broadened to population-based approaches at the community and national level to reduce the mental health disparities among low-SES women on a more permanent basis. 
170 
Summary 
172 


\section{Summary}

Depression is a common psychological disorder that can lead to limitations in social, emotional and physical functioning and that dramatically reduces quality of life. Two groups appear to be particularly affected by this mental health problem, namely women and persons with a low socio-economic status (SES). Especially low-SES women are a vulnerable group at increased risk for depressive symptoms and depression. Numerous factors may contribute to depression in women, including hormonal, genetic, and other biological causes. Also, stress factors play an important role in the onset of depression. The number and types of stressors as well as the mechanisms for coping with stress may all contribute to an adverse mental health outcome. Low-SES women may have the same biological vulnerability for depression as other women, but are more likely to suffer from depression as a result of elevated exposure to stressors, which is socially patterned by socioeconomic status. For instance, disadvantaged women are at high risk of experiencing low income, inadequate housing in dangerous neighborhoods, unemployment, low education, or heavy childcare responsibilities. Also, they have fewer personal and social resources to protect them against the negative effects of stress, such as self-esteem, coping strategies, self-efficacy, personal mastery, and social support. So the increased exposure to stress, together with fewer psychosocial resources, significantly increases the risk of depression in low-SES women. Each of these factors has the potential to be changed and could possibly help to prevent depression.

The central aim of this dissertation was to explore the possibilities for the prevention of depressive symptoms in disadvantaged women. To this aim the first part of this thesis addresses the broader context of preventing depression in low-SES women. This involves questions such as: 'Which women are most likely to benefit from participation in preventive activities', 'What steps should be undertaken to reach this target group', and 'What has already been done to reduce depression in this group?'

Selective and indicated prevention programs target those groups in society whose risk of developing a mental disorder is significantly higher than average or who already display minimal but detectable signs or symptoms of a mental disorder. To identify high-risk groups for participation in depression prevention, risk factors associated with high rates of depressive symptoms are commonly used. However, in certain high-risk populations, such as low-SES women, many are exposed to these risk factors but not all necessarily become depressed. The study reported in Chapter 2 explored which demographic, 
socio-economic and psychological risk factors were associated with selfreported depressive symptoms among our sample of low-SES women. We also investigated whether the numbers of risk factors present in the lives of these women might put them at increased risk. Outcomes indicated that important agents for increased depressive symptom scores were the individual-level SES factors lower education level, lack of current employment and lower net monthly family income. However, the strongest association was found for the psychological risk factor 'perceived stress' which both contributed directly to increased depressive symptom levels, and mediated the outcomes of some of the individual-level SES factors. A further important finding for prevention practice was that the accumulation of risk factors notably increased the chance of depressive symptoms among low-SES women, starting at $12.5 \%$ for women with one risk factor and reaching $71 \%$ for women with six or more risk factors.

Even when we know which low-SES women could best be targeted with preventive action, the depression prevention programs developed for the general public may not always be most suitable for this population. Chapter 3 presented a meta-analysis on psychosocial interventions specifically designed to reduce depressive symptoms in low-SES women. The scope and the overall efficacy of these interventions, both immediate and over time, were investigated together with potential moderators for intervention outcomes. Fourteen studies that had been developed specifically for low-SES women were included in the meta-analysis. The most common preventive interventions were psycho-education, interpersonal therapy and social support strategies. The intervention delivery format was generally in the form of group courses provided by mental health professionals. These studies indicated significant positive effects for the reduction of depressive symptoms in lowSES women, with a weighted mean effect size of 0.31 . This small to moderate effect size is consistent with those found in earlier meta-analyses for depression prevention and mental health promotion. Although follow-up effect sizes were not significant, a trend emerged in which the significant short-term reduction of depressive symptoms declined over time. The study found no evidence for the moderating impact of study sample characteristics, intervention characteristics or research design.

While this meta-analysis showed that a number of promising programs have been specifically developed for low-SES women, effective prevention also depends on our ability to recruit these women for these programs, their willingness to participate, and successful retention. However, there is limited knowledge on which recruitment techniques are most effective for this population or which characteristics in low-SES women are associated with their participation and retention in interventions. Therefore, the study reported 
in Chapter 4 investigated the effectiveness of different strategies for recruiting and retaining low-SES women in depression prevention. This study explored the socio-demographic characteristics and risk status factors within this specific target group that are associated with successful recruitment and retention. Outcomes indicated that active recruitment and screening methods, especially referral through the patient databases of GPs, had the most success in recruiting women for initial contact. Other effective ways to engage women were outreach through local community services and referral persons, supported by the use of carefully selected media. In this study, we were able to reach around $12 \%$ of the target population of low-SES women with a telephone screening, and $3.7 \%$ ultimately participated in the intervention. Of the people who develop depression each year in the Netherlands, only about $1 \%$ participates in preventive interventions. In this study, the reach was almost four times higher than the national level and possibly even higher considering that it was achieved among a low-SES population known to be hard to reach with mental health services. Particularly older women and those with more severe complaints at screening were willing to participate and were retained in the intervention.

The second part of this dissertation presented studies on the implementation and evaluation of Exercise without Worries (EWW), an intervention aimed to reduce stress and depressive symptoms in low-SES women living in disadvantaged neighborhoods by means of a combined psycho-education and exercise program. Previous experiences with exclusively cognitive-oriented depression prevention suggested that these programs have a limited number of low-SES participants, possibly because of their low appeal among this population. The cognitive orientation and written homework assignments included in these training programs leads to frequent drop-out of participants from disadvantaged groups. The EWW course was designed as one way to address this problem by combining psycho-education with an exercise component. EWW targets risk factors for stress and depression in low-SES women through a resiliency-based focus and was adapted to the culture, values, and surroundings of its target population. The intervention's main goal is to reduce stress and depressive complaints and to increase coping related competences through improving the balance between burden and capacity. A core element in the EWW course is its group based format in which the psychoeducative topics are linked with body focused exercises. In each session, psycho-education and exercise components are coordinated as far as possible with the aim of reciprocally reinforcing the message. This is further elaborated 
through four themes (I - Self-image, II - Balance, III - Strength, and IVBoundaries) that form the basis of eight two-hour sessions.

Chapter 5 described the theoretical background and program content of the EWW intervention. A second goal of this chapter was to determine adherence rates, implementation fidelity and subjective evaluations of the program by its intended audience by means of a process evaluation. Outcomes indicated that the implementation of the EWW course went largely according to plan, that the attendance rates were sufficient and that the participants appreciated both the organization and contents of this new course. These findings suggest that a non-stigmatizing approach to exercise and psycho-education fit well with the preferences of low-SES women for preventive intervention.

Besides knowledge on whether a new program is accepted by its target population, it is also necessary to determine whether a prevention program is indeed successful in achieving its intended preventive aims. Chapter $\mathbf{6}$ focused on the efficacy of the EWW intervention and presented its short- and long-term effects on stress and depressive symptoms, as well as moderators of intervention effects. One hundred sixty-one low-SES women with elevated stress or depressive symptom levels were randomly assigned to the combined exercise/psycho-education intervention (EP, $n=57)$, exercise only $(\mathrm{E}, n=51)$ or a wait list control condition (WLC, $n=53$ ). Depressive symptoms (CES-D) and perceived stress (PSS) were measured before and immediately after the intervention and at 2, 6 and 12 month follow-ups. The analyses revealed no differential patterns in reduction of CES-D or PSS scores between the EP, E or WLC groups on the short (post test and 2 months) or long (6 and 12 months) term. Outcome levels of depressive symptoms were moderated by initial depressive symptom scores: Women from the EP and E groups with low initial symptoms benefited from participation in the short term. Further, women in the EP group with the lowest educational level reported more stress reduction than women with higher educational levels. The findings suggest however, that both exercise alone and combined exercise and psycho-education may be a viable prevention option for certain groups of disadvantaged women.

Since there were no indications that EWW reduced depressive symptom and stress levels in the overall target population of low-SES women, the aim of Chapter 7 was to determine the effects of this intervention on the psychosocial program outcomes. It is conceivable that the EWW intervention did lead to changes in these mechanisms, since the course targeted most of these psychosocial factors. General self-efficacy (ALCOS), negative self-statements (ATQ-N), rumination (IBI), self-esteem (NPV-ZW), mastery (PMS), and social support (SSL-I; SSL-D) were measured before and immediately after the intervention and at 6 and 12 month follow-ups. Overall, outcomes suggest that 
offering the EWW course to low-SES women did not produce any beneficial effects on important psychosocial resources associated with risk for depression in this group.

Based on the research presented in this dissertation we can draw some general conclusions on the prevention of depression in low-SES women. First of all, not all disadvantaged women are at equal risk for depression and especially those with an accumulation of socio-economic risk factors, but low symptom scores, could benefit from preventive action. Contrary to popular belief, it is possible to recruit low-SES women who are at risk to participate in depression prevention, although this requires an outreach approach and particular attention to eliminating barriers to participation and retention. While some psychosocial interventions are specifically designed for the reduction of depression in low-SES women, their overall effectiveness requires improvement. Also for the EWW course, several options to improve its effectiveness were noted. While this intervention is acceptable for disadvantaged women, its overall effects on psychosocial outcomes, stress and depressive symptoms are short term and limited to certain groups of participants. It is likely that the adversities that low-SES women encounter in their daily lives slowly erode the effects that can be achieved from participating in indicated prevention efforts. To reduce the mental health disparities among low-SES women on a more permanent basis, the current focus on targeted individual prevention should be broadened to population based approaches at the community and national level. 
178 
Samenvatting 
180 


\section{Samenvatting}

Depressie is een veel voorkomende psychische aandoening die leidt tot beperkingen in het sociale, emotionele en fysieke functioneren en een grote invloed heeft op de kwaliteit van leven. Zowel bij vrouwen als bij mensen met een lage sociaaleconomische status (LSES) komt depressie vaker dan gemiddeld voor. Vooral LSES vrouwen zijn een kwetsbare groep met een groot risico op het ontwikkelen van depressieve klachten en depressie. Bij vrouwen dragen meerdere factoren bij aan het ontstaan van een depressie, waaronder hormonale, genetische en andere biologische oorzaken. Daarnaast spelen stress factoren een belangrijke rol bij het ontstaan van depressie. Het aantal en soort stressoren en de manier waarop met de stress wordt omgegaan kunnen allemaal leiden tot negatieve gevolgen voor de geestelijke gezondheid. Hoewel LSES vrouwen dezelfde genetische en biologische kwetsbaarheid voor depressie hebben als andere vrouwen, is de kans dat ze een depressie ontwikkelen groter doordat ze vaker te maken hebben met een opeenstapeling van stressoren. Deze stressoren hangen dikwijls samen met hun lage sociaaleconomische status, zoals een laag inkomen, slechte woonomstandigheden en buurtproblemen, werkloosheid, een lage opleiding of zware opvoedingstaken. Daarnaast hebben ze minder psychosociale vaardigheden die kunnen helpen bij het tegengaan van de negatieve effecten van stress, zoals zelfwaardering, coping strategieën, eigeneffectiviteit, een positieve beheersingsoriëntatie en sociale steun. Samenvattend hebben LSES vrouwen een aanzienlijk verhoogd risico op depressie door hun grotere blootstelling aan stressoren in samenhang met minder psychosociale vaardigheden om met die stressoren om te gaan. Echter, deze factoren hebben de potentie om te worden veranderend en kunnen zo bijdragen aan de preventie van depressie.

De centrale doelstelling van dit proefschrift is het verkennen van de mogelijkheden om depressieve symptomen bij kansarme vrouwen te voorkómen. Het eerste deel van dit proefschrift richt zich op de bredere context van depressie preventie bij LSES vrouwen. Er wordt ingegaan op vragen als: 'Welke vrouwen zouden het meest baat hebben bij deelname aan preventieve activiteiten', 'Welke stappen moeten worden ondernomen om deze doelgroep te bereiken', en 'Wat is er al gedaan om de depressie te verminderen in deze groep?'

Selectieve en geïndiceerde preventie programma's richten zich op groepen in de samenleving met een hoger dan gemiddeld risico op het ontwikkelen van een psychische stoornis, of die al lichte of subklinische symptomen vertonen van een psychische stoornis. Voor het identificeren van hoogrisico groepen die 
mogelijk baat kunnen hebben van deelname aan depressie preventie, worden vaak risicofactoren gebruikt die samenhangen met een verhoogd niveau van depressieve symptomen. Echter, in sommige groepen, zoals LSES vrouwen, zijn de meeste mensen blootgesteld aan deze risicofactoren, terwijl ze niet allemaal depressief worden. Hoofdstuk 2 beschrijft een studie waarin bij een steekproef van LSES vrouwen is nagegaan welke demografische, sociaaleconomische en psychologische risicofactoren samenhangen met hun zelfgerapporteerde depressieve symptomen. Ook is onderzocht of het aantal aanwezige risicofactoren bijdraagt aan een cumulatief risico voor depressieve klachten. De resultaten geven aan dat de belangrijke risicofactoren voor depressieve symptomen bij LSES vrouwen gerelateerd zijn aan hun individuele sociaaleconomische status, namelijk een lagere opleiding, werkeloosheid en een lager netto maandelijks gezinsinkomen. De sterkste associatie werd echter gevonden voor de psychologische risicofactor 'ervaren stress'. Deze risicofactor draagt niet alleen rechtstreeks bij aan een verhoogd niveau van depressieve klachten, maar medieert ook een aantal individuele SES factoren. Een andere belangrijke conclusie voor de praktijk van depressie preventie is dat bij LSES vrouwen de kans op depressieve symptomen cumulatief toeneemt van 12,5\% bij personen met maar één risicofactor tot $71 \%$ bij vrouwen die zijn blootgesteld aan zes of meer risicofactoren.

Hoewel uit het voorgaande hoofdstuk blijkt dat bepaalde groepen LSES vrouwen baat kunnen hebben bij deelname aan preventieve activiteiten, zijn de beschikbare programma's niet altijd geschikt voor deze doelgroep. Hoofdstuk 3 presenteert een meta-analyse van psychosociale interventies die specifiek zijn ontworpen om depressieve symptomen bij LSES vrouwen te verminderen of te voorkomen. De inhoud van deze interventies en hun effectiviteit op korte en lange termijn werd onderzocht, evenals mogelijke moderatoren voor interventie effectiviteit. De meta-analyse omvatte veertien studies die speciaal gericht zijn op LSES vrouwen. De meest voorkomende interventiemethoden waren psycho-educatie, inter-persoonlijke therapie en sociale steun strategieën. De interventies worden over het algemeen in de vorm van groepscursussen aangeboden en begeleid door professionals uit de geestelijke gezondheidszorg. De studies lieten significant positieve effecten zien voor de afname van depressieve klachten bij LSES vrouwen, met een gewogen gemiddelde effectgrootte van 0,31. Deze kleine tot gemiddelde effectgrootte komt overeen met uitkomsten van eerdere meta-analyses op het gebied van depressiepreventie en geestelijke gezondheid bevordering. Hoewel de effectgroottes op langere termijn niet meer significant waren, was een trend te zien waarbij de korte termijn verbetering van depressieve klachten na loop van tijd afzwakte. De studie vond geen bewijs voor modererende invloed van 
kenmerken van de studiepopulaties, interventies of de gebruikte onderzoeksopzet.

Hoewel de meta-analyse laat zien dat programma's specifiek ontwikkeld voor LSES vrouwen veelbelovend zijn, hangt effectieve preventie ook af van hoe goed we in staat zijn om deze groep vrouwen te werven, hun bereidheid tot deelname, en hun aanwezigheid tijdens de uitvoering van een interventie. Echter, we hebben onvoldoende kennis over welke technieken het meest effectief zijn om LSES vrouwen te werven en welke kenmerken van deze groep samenhangen met hun deelname en behoud. Hoofdstuk 4 rapporteert de uitkomst van een onderzoek naar de effectiviteit van verschillende strategieën voor het werven van LSES vrouwen en hun deelname aan depressiepreventie. Deze studie onderzocht welke socio-demografische en risico kenmerken geassocieerd zijn met succesvolle werving en behoud van deze specifieke doelgroep. De resultaten geven aan dat actieve werving en screening methoden, in het bijzonder het gebruik van patiëntbestanden van huisartsen, het vaakst leiden tot een intake gesprek. Andere effectieve manieren om vrouwen te bereiken zijn actieve werving door lokale zorg- en welzijnsinstanties of verwijzers, ondersteunt door advertenties in zorgvuldig geselecteerde media. In deze studie bereikten we ongeveer $12 \%$ van onze LSES doelgroep voor een telefonische screening, 3,7\% nam uiteindelijk deel aan de interventie. Van alle Nederlanders die jaarlijks aan een depressie lijden participeert slechts ongeveer $1 \%$ in preventieve interventies. In deze studie is het bereik bijna vier maal zo hoog als het landelijk gemiddelde. Mogelijk is dit bereik cijfer nog hoger gezien het feit dat we ons richten op een LSES doelgroep waarvan bekend is dat deze moeilijk te bereiken is met geestelijke gezondheidszorg. Vooral oudere vrouwen en vrouwen die bij de screening meer depressieve klachten hadden waren bereid om deel te nemen aan de interventie en trokken zich hier minder vaak uit terug.

Het tweede deel van dit proefschrift presenteert studies over de uitvoering en evaluatie van Bewegen Zonder Zorgen (BZZ). Dit is een psycho-educatief bewegingsprogramma dat beoogt om stress en depressieve symptomen bij LSES vrouwen uit achterstandswijken te voorkomen en te verminderen. Ervaringen met uitsluitend cognitief georiënteerde depressie preventie cursussen laten zien dat deze interventies vaak niet laagdrempelig genoeg zijn voor LSES groepen. Het cursusniveau en het huiswerk worden te moeilijk gevonden en leiden tot meer uitval onder deelnemers met een lager opleidingsniveau. De BZZ cursus is ontworpen om dit probleem aan te pakken door een combinatie van psycho-educatie met lichamelijke inspanning. BZZ richt zich op risicofactoren voor stress en depressie bij LSES vrouwen en is 
laagdrempelig omdat de structuur en de moeilijkheidsgraad zijn aangepast aan de vaardigheden van de doelgroep. Het preventieve einddoel van BZZ is het verminderen van stress en depressieve klachten en het vergroten van coping vaardigheden door het in balans brengen van draaglast en draagkracht. Een kernelement van BZZ is het werken in groepen waarbij de psycho-educatieve onderwerpen aansluiten bij de beweging activiteiten. In iedere bijeenkomst worden de bewegingsoefeningen en psycho-educatie zo veel mogelijk gecoördineerd, zodat de deelneemsters niet alleen spreken over een onderwerp, maar het ook ervaren door middel van de oefeningen. Deze opzet wordt verder uitgewerkt in de vier thema's (I - Zelfbeeld, II - Balans, III Kracht, en IV - Grenzen) die de basis vormen voor de acht bijeenkomsten van twee uur.

Hoofdstuk 5 beschrijft de theoretische achtergrond en programma-inhoud van de BZZ interventie. Dit hoofdstuk heeft verder tot doel om via een proces evaluatie te bepalen wat de deelnamepercentages zijn, hoe het met de implementatie trouw gesteld is en hoe de doelgroep het programma beoordeelt. De uitkomsten geven aan dat de uitvoering van BZZ grotendeels volgens plan is verlopen, de deelname percentages waren voldoende en de deelneemsters waardeerden zowel de opzet als de inhoud van deze nieuwe cursus. Deze bevindingen suggereren dat de non-stigmatiserende aanpak van bewegingsactiviteiten in combinatie met psycho-educatie aansluit bij de wensen van LSES vrouwen voor preventieve interventies.

Naast kennis over hoe de doelgroep een nieuw preventieprogramma beoordeelt, is het ook noodzakelijk om te bepalen of een programma inderdaad zijn beoogde preventieve doelstellingen bereikt. Hoofdstuk 6 richt zich op de effectiviteit van de BZZ interventie en presenteert de korte- en lange- termijn effecten op stress en depressieve symptomen en mogelijke effectmoderatoren. Honderd een en zestig LSES vrouwen met stressklachten of depressieve symptomen werden willekeurig toegewezen aan de interventie met bewegingsoefeningen en psycho-educatie (BP, $n=57$ ), alleen de bewegingsoefeningen ( $\mathrm{B}, n=51$ ) of een wachtlijstcontrolegroep (WLC, $n=53$ ). Depressieve symptomen (CES-D) en ervaren stress (PSS) werden vóór en onmiddellijk na de interventie gemeten en 2, 6 en 12 maanden later. Zowel op de korte termijn (post-test en 2 maanden follow-up) als de lange termijn (6 en 12 maanden follow-up) waren er geen verschillen te zien tussen de BP, B en WLC groepen in de mate waarmee de depressieve of stress klachten waren afgenomen. De uitkomsten voor depressieve symptomen werden gemodereerd door de scores bij aanvang: deelneemsters aan de BP en B groepen met weinig symptomen bij aanvang van de cursus profiteerden op korte termijn van deelname. Verder rapporteerden vrouwen uit de BP groep met een laag 
opleidingsniveau na afloop meer afname van stress klachten dan deelneemsters met hogere opleidingsniveaus. Deze bevindingen suggereren dat bewegingsoefeningen, alleen óf in combinatie met psycho-educatie, een preventief effect kunnen hebben bij bepaalde groepen LSES vrouwen.

Aangezien er geen aanwijzingen zijn dat BZZ bijdraagt aan afname van depressieve symptomen en stress klachten in de totale doelgroep van de LSES vrouwen, is het doel van Hoofdstuk 7 om te bepalen of deze interventie effecten heeft op hun psychosociale vaardigheden. Het is mogelijk dat de BZZ interventie bijdraagt aan veranderingen in deze vaardigheden, omdat de cursus zich expliciet richt op deze psychosociale factoren. Algemene eigeneffectiviteit (ALCOS), negatieve gedachten (ATQ-N), piekeren (IBI), zelfwaardering (NPV-ZW), positieve beheersingsoriëntatie (PMS), en sociale steun (SSL-I; SSL-D) werden gemeten vóór en onmiddellijk na de interventie en 6 en 12 maanden later. De uitkomsten laten zien dat het aanbieden van de BZZ cursus aan LSES vrouwen geen positieve veranderingen produceert in psychosociale vaardigheden die geassocieerd zijn met hun verhoogde risico op depressie.

Op basis van het onderzoek beschreven in dit proefschrift kunnen we enkele algemene conclusies te trekken over de preventie van depressie bij vrouwen met een lage sociaaleconomische status. Ten eerste hebben niet alle kansarme vrouwen hetzelfde risico op depressie. Vooral de vrouwen met een accumulatie van sociaaleconomische risicofactoren, maar die nog weinig symptomen vertonen, kunnen profiteren van preventieve maatregelen. In tegenstelling tot wat vaak wordt gedacht is het mogelijk aan LSES vrouwen te werven voor deelname aan depressiepreventie, hoewel dit een actieve aanpak vereist met expliciete aandacht voor het wegnemen van barrières die deelname kunnen belemmeren. Hoewel er steeds meer psychosociale interventies worden ontworpen met het specifieke doel om depressie bij LSES vrouwen te verminderen, kan hun algemene effectiviteit nog verder verbeterd worden. Ook voor Bewegen Zonder Zorgen zijn verschillende suggesties gegeven om de doeltreffendheid van de cursus te verbeteren. Hoewel de inhoud van deze interventie gewaardeerd wordt door LSES vrouwen, zijn de effecten op psychosociale vaardigheden, stress en depressieve symptomen slechts van korte termijn en beperkt tot bepaalde groepen deelneemsters. De moeilijke omstandigheden waarmee LSES vrouwen in hun dagelijkse leven worden geconfronteerd kunnen mogelijk de effecten van hun deelname aan geïndiceerde preventie activiteiten doen afnemen. Om de geestelijke gezondheid van LSES vrouwen op een meer structurele manier te verbeteren, moet de huidige focus van individuele preventie in specifieke doelgroepen 
worden aangevuld met een populatie brede aanpak met interventies op zowel lokaal als nationaal niveau. 
Résumé 
188 


\section{Résumé}

La dépression est un trouble psychologique répandu qui peut conduire à des limitations dans le fonctionnement social, affectif et physique et qui réduit considérablement la qualité de vie. Deux groupes sont particulièrement affectés par ce problème de santé mentale, notamment les femmes et les personnes ayant un faible statut socio-économique (SSE). Plus précisément, les femmes à faible SSE forment un groupe vulnérable aux risques plus élevés de symptômes dépressifs et de dépression. De nombreux facteurs peuvent contribuer à la dépression chez les femmes, parmi elles les causes d'origine hormonale, génétique ou biologique. Aussi, les facteurs de stress jouent un rôle important à l'origine de la dépression. Le nombre et la variété de ces facteurs, ainsi que les mécanismes pour faire face au stress, peuvent tous contribuer à des troubles de la santé mentale. Les femmes à faible SSE peuvent avoir les mêmes vulnérabilités d'ordre biologique à la dépression que les autres femmes, mais ont plus de probabilités d'en souffrir en raison d'une exposition plus élevée aux facteurs de stress liés à leur statut socio-économique. Par exemple, les femmes défavorisées ont plus de risque d'avoir des revenus bas, habiter des logements inadéquats dans des quartiers dangereux, vivre des périodes prolongées de chômage, avoir un faible niveau d'éducation, ou rencontrer des difficultés dans l'éducation de ses enfants. En outre, elles ont moins de ressources intérieures et sociales pour les protéger contre les effets négatifs du stress, telles que l'estime de soi, les stratégies pour faire face aux problèmes, l'auto-efficacité, la maîtrise personnelle et le soutien social. Ainsi, l'exposition accrue au stress, avec moins de ressources psychosociales, augmente significativement le risque de dépression chez les femmes à faible SSE. Chacun de ces facteurs a le potentiel d'être changé et pourrait aider à prévenir la dépression.

L'objectif principal de cette thèse était d'explorer les possibilités de la prévention des symptômes dépressifs chez les femmes défavorisées. Dans cette optique, la première partie de cette thèse aborde le contexte plus large de la prévention de la dépression chez les femmes à faible SSE. On y aborde des questions telles que: 'Quelles sont les femmes plus susceptibles de bénéficier de la participation à des activités préventives', 'Quelles mesures devraient être prises pour atteindre ce groupe cible', et 'Qu'est ce qu'a déjà été fait pour réduire la dépression chez les membres de ce groupe?'

Les programmes de prévention sélective et indiquée ciblent ces groupes dans la société dont le risque de développer un trouble mental est beaucoup plus élevé que la moyenne ou qui présentent déjà des symptômes légers d'un 
trouble mental. Pour identifier les groupes à haut risque susceptibles de bénéficier de la prévention de la dépression, des facteurs de risque associés à des taux élevés de symptômes dépressifs sont communément utilisés. Toutefois, dans certaines populations à haut risque, comme les femmes défavorisées, un certain nombre de personnes sont exposées à ces facteurs de risque mais pas toutes ne deviennent nécessairement déprimés. L'étude rapportée dans le Chapitre $\mathbf{2}$ a exploré quels facteurs de risque démographiques, socio-économiques et psychologiques ont été associés à des symptômes dépressifs auto-rapportés chez notre échantillon de femmes à faible SSE. On a également cherché à savoir si le nombre de facteurs de risque présents dans la vie de ces femmes pourrait les mettre face à un risque accru. Les résultats indiquent que les agents importants pour les symptômes dépressifs élevés étaient les facteurs socio-économiques au niveau individuel: un niveau de scolarité inférieur, une situation de chômage et de faibles revenus. Toutefois, la plus forte association a été trouvé pour le facteur de risque psychologique désigné sous le terme de 'stress perçu', qui a contribué à la fois aux symptômes dépressifs élevés, mais qui a aussi joué un rôle de médiateur sur les résultats de certaines facteurs SSE au niveau individuel. Une autre constatation importante dans la pratique de la prévention a été qu'une accumulation des facteurs de risque a augmenté en particulier le risque de symptômes dépressifs chez les femmes défavorisées, partant de $12,5 \%$ pour les femmes n'ayant qu'un facteur de risque, jusqu'à 71\% pour les femmes avec six ou plusieurs facteurs de risque.

Même lorsque l'on sait quelles femmes défavorisées pourraient être le mieux ciblées avec des mesures préventives, les programmes de prévention de la dépression développés pour le grand public ne peuvent pas toujours être les plus appropriés pour cette population. Le Chapitre 3 a présenté une métaanalyse sur les interventions psychosociales spécifiquement conçues pour réduire les symptômes dépressifs chez les femmes de faible statut socioéconomique. La portée et l'efficacité globale de ces interventions, immédiate et à long terme, ont été étudiées en collaboration avec les modérateurs potentiels pour les résultats des interventions. Quatorze études ayant été développées spécifiquement pour les femmes à faible SSE ont été incluses dans la métaanalyse. Les interventions préventives les plus courantes ont été la psychoéducation, la thérapie interpersonnelle et les stratégies de soutien social. L'exécution des interventions a été généralement sous la forme de cours collectifs rédigés par des professionnels de la santé mentale. Ces études ont montré des effets positifs et significatifs pour la réduction des symptômes dépressifs chez les femmes de faible SSE, avec une taille d'effet moyenne pondérée de 0,31 . Cette taille d'effet faible à modérée est conforme à celles 
trouvées dans les méta-analyses pour la prévention de la dépression et la promotion de la santé mentale. Bien que les tailles d'effets de suivi ne étaient pas significatives, une tendance est apparue dans lequel la réduction significative des symptômes dépressifs à court terme a diminué au fil du temps. L'étude n'a trouvé aucune preuve d'un effet modérateur des caractéristiques de l'échantillon d'étude, les caractéristiques d'intervention ou de conception de la recherche.

Bien que cette méta-analyse ait montré qu'un certain nombre de programmes prometteurs ont été développés spécifiquement pour les femmes défavorisées, la prévention efficace dépend aussi de notre capacité à recruter ces femmes à ces programmes, leur volonté de participer et le succès du maintien de leur participation. Cependant, il y a une connaissance limitée par rapport aux techniques de recrutement, a savoir quelles sont les plus efficaces pour ce type de population, ou quelles caractéristiques parmi les femmes à faible SSE favorisent leur participation et la rétention dans les interventions. Par conséquent, l'étude rapportée dans le Chapitre 4 a examiné l'efficacité de différentes stratégies pour recruter et fidéliser les femmes défavorisées pour la prévention de la dépression. Cette étude a exploré les caractéristiques sociodémographiques et les facteurs de risques au sein de ce groupe cible spécifique qui sont associés au recrutement et à la rétention. Les résultats ont indiqué que les méthodes de recrutement et de dépistages actif, en particulier les bases de données des patients des médecins généralistes, donnent les meilleurs résultats pour créer un premier contact. D'autres moyens efficaces pour engager les femmes ont été les services communautaires locaux et les personnes de référence, soutenue par l'utilisation des médias soigneusement sélectionnés. Dans cette étude, nous avons pu atteindre environ $12 \%$ de la population cible des femmes défavorisées avec un dépistage par téléphone, et $3,7 \%$ a participé à l'intervention en fin de compte. Parmi les personnes qui développent une dépression chaque année aux Pays-Bas, environ $1 \%$ seulement participe à des interventions préventives. Dans cette étude, la portée a été presque quatre fois plus élevé que le niveau national et peut-être encore plus élevé étant donné qu'il a été obtenu auprès d'une population à faible SSE connue pour être difficile à atteindre avec les services de santé mentale. En particulier, les femmes plus âgées et celles avec plus de symptômes au dépistage ont été disposées à participer et ont été retenus dans l'intervention.

La deuxième partie de cette thèse a présenté des études sur l'implémentation et l'évaluation de l'Exercice Sans Soucis (ESS), une intervention combinant la psychoéducation et l'exercice visant à réduire les symptômes de stress et de dépression auprès les femmes à faible SSE vivant dans des quartiers 
défavorisés. Les expériences antérieures sur la prévention de la dépression exclusivement orientée vers le cognitif suggèrent que les participants à faible SSE à ces programmes sont limités, probablement en raison d'une faible attractivité au sein de cette population. L'orientation cognitive et les devoirs écrits dans ces programmes conduisent à l'abandon fréquent des participants issus de groupes défavorisés. Le cours ESS été conçu comme un moyen de résoudre ce problème en combinant la psychoéducation avec l'exercice. ESS est ciblé sur les facteurs de risque du stress et de la dépression chez les femmes défavorisées en mettant l'accent sur la résistance et a été adapté à la culture, les valeurs, et l'environnement de sa population cible. L'objectif principal de cette intervention est de réduire les symptômes de stress et de dépression et de renforcer les compétences adaptatives par l'amélioration de l'équilibre entre la charge et la capacité. Un élément essentiel dans le cours ESS est son format en fonction du groupe dans lequel les sujets psycho-éducatifs sont liées aux exercices. Dans chaque session, la psychoéducation et les exercices sont coordonnés autant que possible dans le but de renforcer la réciprocité des messages. Cette réflexion est développée à travers quatre thèmes (I - Image de soi, II - Balance, III - Force, et IV - Limites) qui forment la base de huit séances de deux heures.

Le Chapitre 5 décrit les bases théoriques et le contenu de l'intervention ESS. Un deuxième objectif de ce chapitre était de déterminer le taux d'adhérence, ainsi que de la fidélité de l'implémentation et des évaluations subjectives du programme par son public cible au moyen d'une évaluation de processus. Les résultats indiquent que la mise en œuvre du cours ESS ont suivi strictement les planifications, que le taux d'adhérence était suffisant et que les participants ont apprécié l'organisation et le contenu de ce nouveau cours. Ces résultats suggèrent que l'approche non stigmatisante de l'exercice et la psychoéducation s'accordent aux préférences des femmes défavorisées dans le cadre d'une intervention préventive.

En outre de savoir si un nouveau programme est accepté par sa population cible, il est également nécessaire de déterminer si un programme préventif atteint en effet ses objectifs de prévention. Le Chapitre 6 est axé sur l'efficacité de l'intervention ESS et a présenté ses effets à court et long terme sur les symptômes de stress et de dépression, ainsi que les modérateurs des effets. Cent soixante et une femmes à faible SSE avec des symptômes élevés de stress ou de la dépression ont été assignés au hasard à l'intervention combinée exercice et psychoéducation (EP, $n=57$ ), seul exercice $(E, n=51$ ) ou une condition de contrôle en forme de liste d'attente (CLA, $n=53$ ). Les symptômes dépressifs (CES-D) et le stress perçu (PSS) ont été mesurés avant et immédiatement après l'intervention et après 2 , 6 et 12 mois de suivi. Les 
analyses n'ont révélé aucune différence dans la de réduction de la CES-D ou des scores PSS entre les groupes EP, E ou CLA à court terme (post-test et 2 mois) ou à long terme (6 et 12 mois). Ces résultats au niveau de symptômes dépressifs ont été modérés par des symptômes dépressifs initiaux: les femmes des groupes EP et E à faible symptômes initiaux ont bénéficié de leur participation à ESS dans le court terme. En outre, les femmes dans le groupe de l'EP avec le plus bas niveau d'éducation ont rapporté plus de réductions de stress que des femmes à des niveaux élevés d'éducation. Les résultats suggèrent cependant que l'exercice tout seul ou combiné avec la psychoéducation peuvent être une option préventive viable pour certains groupes de femmes défavorisées.

Comme il n'y avait aucune indication qu'ESS réduit les symptômes de stress ou de dépression dans la population cible globale des femmes défavorisées, l'objectif du Chapitre 7 a été de déterminer les effets de cette intervention sur les résultats psychosociaux. Il est concevable que l'intervention ESS ait entraîné des changements dans ces mécanismes, étant donné que le cours cible la plupart de ces facteurs psychosociaux. L'auto-efficacité générique (ALCOS), les assertions de soi-même négatives (ATQ-N), la rumination (IBI), l'estime de soi (NPV-ZW), la maîtrise (PMS), et le soutien social (SSL-I; SSL-D) ont été mesurée avant et immédiatement après l'intervention et après 6 et 12 mois de suivi. Dans l'ensemble, les résultats suggèrent que le cours ESS ne produise pas d'effets bénéfiques sur d'importantes ressources psychosociales qui sont liées à un risque à la dépression chez des femmes défavorisées.

Sur la base des travaux de recherche présentés dans cette thèse, nous pouvons tirer quelques conclusions générales sur la prévention de la dépression chez les femmes à faible statut socio-économique. Tout d'abord, les femmes défavorisées n'ont pas toutes un risque égal de dépression et surtout celles accumulant les facteurs de risque socio-économiques, présentant cependant des symptômes pas trop élevés, pourraient tirer un bénéfice de mesures préventives. Contrairement aux pensées instinctives, il est possible de recruter des femmes à faible SSE avec un risque élevé et de les faire participer à la prévention de la dépression. Cela nécessite une approche de sensibilisation et une attention particulière à l'élimination des obstacles à leur participation et la rétention des candidates. Bien que certaines interventions psychosociales soient spécifiquement conçues pour la réduction de la dépression chez les femmes à faible SSE, leur efficacité globale nécessite une amélioration. Aussi pour le cours ESS, plusieurs options pour améliorer son efficacité ont été notées. Bien que cette intervention est acceptable pour les femmes défavorisées, ses effets globaux sur les résultats psychosociaux, le stress et les symptômes 
dépressifs sont de courte durée et limités à certains groupes de participantes. Il est probable que les difficultés quotidiennes rencontrées par les femmes à faible SSE érodent graduellement les effets qui peuvent être atteints après une participation aux efforts de prévention proposés. Des pistes existent pour améliorer de façon plus permanente la santé mentale parmi les femmes défavorisées. En effet, la prévention qui est actuellement individuelle devrait être élargie à des groupes entiers de population, en particulier au niveau communautaire et à l'échelon national. 
References 
196 


\section{References}

Adler, N. E., \& Rehkopf, D. H. (2008). U.S. disparities in health: Descriptions, causes, and mechanisms. Annual Review of Public Health, 29, 235-252.

Albee, G. W. (1986). Toward a just society: Lessons from observations on the primary prevention of psychopathology. American Psychologist, 41, 891-897.

Albee, G. W. (1996). Revolutions and counterrevolutions in prevention. American Psychologist, 51, 1130-1133.

Albright, C. L., Pruitt, L., Castro, C., Gonzalez, A., Woo, S., \& King, A. C. (2005). Modifying physical activity in a multiethnic sample of low-income women: One-year results from the IMPACT (Increasing Motivation for Physical ACTivity) project. Annals of Behavioural Medicine, 30, 191-200.

Alderman, B. L., Rogers, T. J., Johnson, T. A., \& Landers, D. M. (2003). A meta-analysis of exercise and stress reactivity. Medicine \& Science in Sports \& Exercise, 35, S26.

Alegria, M., Bijl, R. V., Lin, E., Walters, E. E., \& Kessler, R. C. (2000). Income differences in persons seeking outpatient treatment for mental disorders. Archives of General Psychiatry, 57, 383-391.

Ali, B. S., Rahbar, M. H., Naeem, S., Gul, A., Mubeen, S., \& Iqbal, A. (2003). The effectiveness of counseling on anxiety and depression by minimally trained counselors: a randomized controlled trial. American Journal of Psychotherapy 57, 324-336.

Allart-van Dam, E., \& Hosman, C. M. H. (2002). De invloed van sociaal-economische status en sekse op het effect van de 'Omgaan met depressie' cursus. [The effects of socio-economic status and gender on the effects of the 'Coping with Depression' course.] Tijdschrift voor Gezondheidswetenschappen, 80, 262-268.

Allart-van Dam, E., Hosman, C. M. H., Hoogduin, C. A. L., \& Schaap, C. P. D. R. (2003). The Coping with depression course: Short- term outcomes and mediating effects of a randomized controlled trial in the threatment of subclinical depression. Behaviour Therapy, 34, 381-396.

Allart-van Dam, E., Hosman, C. M. H., Hoogduin, C. A. L., \& Schaap, C. P. D. R. (2007). Prevention of depression in subclinically depressed adults: Follow-up effects on the 'Coping with Depression' course. Journal of Affective Disorders, 97, 219-228.

Almeida, O.P., Alfonso, H., Pirkis, J., Kerse, N., Sim, M., Flicker, L., Snowdon, J., Draper, B., Byrne, G., Goldney, R., Lautenschlager, N.T., Stocks, N., Scazufca, M., Huisman, M., Araya, R., \& Pfaff, J. (2010). A practical approach to assess depression risk and to guide risk reduction strategies in later life. International Psychogeriatrics, Doi:10.1017/S1041610210001870.

Almeida-Filho, N., Lessa, I., Magalhaes, L., Araujo, M. J., Aquino, E., James, S. A., \& Kawachi, I. (2004). Social inequality and depressive disorders in Bahia, Brazil: Interactions of gender, ethnicity, and social class. Social Science \& Medicine, 59, 1339-1353.

Alvidrez, J. (1999). Ethnic variations in mental health attitudes and service use among low-income African American, Latina, and European American young women. Community Mental Health Journal, 35, 515-530.

Alvidrez, J., \& Azocar, F. (1999). Distressed women's clinic patients: Preferences for mental health treatments and perceived obstacles. General Hospital Psychiatry, 21, 340-347.

Andersen, I., Thielen, K., Nygaard, E., \& Diderichsen, F. (2009). Social inequality in the prevalence of depressive disorders. Journal of Epidemiology and Community Health, 63, 575-81.

Anderson, C. M., Robins, C. S., Greeno, C. G., Cahalane, H., Copeland, V. C., \& Andrews, R. M. (2006). Why lower income mothers do not engage with the formal mental health care system: Perceived barriers to care. Qualitative Health Research, 16, 926-943.

Anderson, R. E., Wadden, T. A., Bartlett, S. J., Zemmerl, B., Verde, T. J., \& Franckowiak, S. C. (1999). Effects of lifestyle activity vs structured aerobic in obese women: a randomized trial. JAMA, 281, 335-340.

Andresen, E. M., Malmgren, J. A., Carter, W. B., \& Patrick, D. L. (1994). Screening for depression in well older adults: Evaluation of a short form of the CES-D (Center for Epidemiologic Studies Depression Scale). American Journal of Preventive Medicine, 10, 77-84.

Aneshensel, C. S. (1992). Social stress: Theory and research. Annual Review of Sociology, 18, 15-38. 
Aneshensel, C. S. (2009). Toward explaining mental health disparities. Journal of Health and Social Behavior 50, 377-394.

Aneshensel, C. S., Frerichs, R. R., Clark, V. A., \& Yokopenic, P. A. (1982). Measuring depression in the community: A comparison of telephone and personal interviews. Public Opinion Quarterly, $46,110-121$.

Aquilino, W. S. (1998). Effects of interview mode on measuring depression in younger adults. Journal of Official Statistics, 14, 15-29.

Araya, R., Lewis, G., Rojas, G., \& Fritsch, R. (2003). Education and income: Which is more important for mental health? Journal of Epidemiology and Community Health, 57, 501-505.

Araya, R., Rojas, G., Fritsch, R., Caete, J., Rojas, M., Simon, G., \& Peters, T. J. (2003). Treating depression in primary care in low- income women in Santiago, Chile: A randomized controlled trial. Lancet, 361, 995-1000.

Arean, P. A., \& Miranda, J. (1996). Do primary care patients accept psychological treatments? General Hospital Psychiatry, 18, 22-27.

Arean, P. A., Alvidrez, J., Nery, R., Estes, C., \& Linkins, K. (2003). Recruitment and retention of older minorities in mental health services research. The Gerontologist, 43, 36-44.

Aro, A., R, Nyberg, N., Absetz, P., Hendriksson, M., \& Lönnqvist, J. (2001). Depressive symptoms in middle-aged women are more strongly associated with physical health and social support than with socioeconomic factors. Nordic Journal of Psychiatry, 55, 191-198.

Atlantis, E., Chow, C. M., Kirby, A., \& Singh, M. F. (2004). An effective exercise-based intervention for improving mental health and quality of life measures: A randomized controlled trial. Preventive Medicine, 39, 424-434.

Ayuso-Mateos, J. L., Pereda, A., Dunn, G., Vazquez-Barquero, J. L., Casey, P., Lehtinen, V., Dalgard, O., Wilkinson, G., \& Dowrick, C. (2007). Predictors of compliance with psychological interventions offered in the community. Psychological Medicine, 37, 717-725.

Azocar, F., Miranda, J., \& Dwyer, E. V. (1996). Treatment of depression in disadvantaged women. Women and Therapy, 18, 91-105.

Baas, K.D., Wittkampf, K.A., Van Weert, H.C., Lucassen, P., Huyser, J., Van den Hoogen, H., Van de Lisdonk, E., Bindels, P.E., Bockting, C.L., Ruhe, H.G., \& Schene, A.H. (2009). Screening for depression in high-risk groups: Prospective cohort study in general practice. The British Journal of Psychiatry, 194, 399-403.

Bakermans-Kranenburg, M. J., Van IJzendoorn, M. H., \& Juffer, F. (2003). Less is more: Metaanalyses of sensitivity and attachment interventions in early childhood. Psychological Bulletin, $129,195-215$.

Ball, K., Salmon, J., \& Crawford, D. (2006). How can socio-economic differences in physical activity among women be explained? Women and Health, 43, 93-113.

Bandura, A. (1997). Self-efficacy: The exercise of control. New York: W.H. Freeman.

Banyard, V. L., \& Graham-Bermann, S. A. (1998). Surviving poverty: Stress and coping in the lives of housed and homeless mothers. American Journal of Orthopsychiatry, 68, 479-489.

Barbour, K. A., Edenfield, T. M., \& Blumenthal, J. A. (2007). Exercise as a treatment for depression and other psychiatric disorders. Journal of cardiopulmonary rehabilitation and prevention, 27, 359367.

Baron, R.M., \& Kenny, D. A. (1986). The moderator-mediator variable distinction in social psychological research: Conceptual, strategic, and statistical considerations. Journal of Personality and Social Psychology 51, 1173-1182.

Bassuk, E. L., Buckner, J. C., Perloff, J. N., \& Bassuk, S. S. (1998). Prevalence of mental health and substance use disorders among homeless and low-income housed mothers. American Journal of Psychiatry, 155, 1561-1564.

Bassuk, E. L., Mickelson, K. D., Bissell, H. D., \& Perloff, J. N. (2002). Role of kin and nonkin support in the mental health of low-income women. American Journal of Orthopsychiatry, 72, 39-49.

Baum, A., Garofalo, J.P., \& Yali, A.M. (1999). Socioeconomic status and chronic stress: Does stress account for SES effects on health? Annals of the New York Academy of Sciences, 896, 131-144.

Baxter, A., \& Kahn, J.V. (1999). Social support, needs and stress in urban families with children enrolled in an early intervention program. Infant Toddler Intervention, 9, 239-257. 
Beeber, L. S., Cooper, C., van Noy, B. E., Schwartz, T. A., Blanchard, H. C., Canuso, R., Robb, K., Laudenbacher, C., \& Emory, S. L. (2007). Flying under the radar: Engagement and retention of depressed low-income mothers in a mental health intervention. Advances in Nursing Science, 30, 221-234.

Beeber, L. S., Holditch-Davis, D., Belyea, M. J., Funk, S. G., \& Canuso, R. (2004). In-home intervention for depressive symptoms with low- income mothers of infants and toddlers in the United States. Health Care for Women International, 25, 561-580.

Beeber, L. S., Perreira, K. M., \& Schwartz, T. (2008). Supporting the mental health of mothers raising children in poverty: How do we target them for intervention studies? Annals of the New York Academy of Sciences, 1136, 86-100.

Beekman, A. T., Penninx, B. W., Deeg, D. J., De Beurs, E.., Geerling, S. W., \& Van Tilburg, W. (2002). The impact of depression on the well-being, disability and use of services in older adults: A longitudinal perspective. Acta Psychiatrica Scandinavica, 105, 20-27.

Belle, D. (1990). Poverty and women's mental health. American Psychologist 45, 385-389.

Belle, D., \& Doucet, J. (2003). Poverty, inequality and discrimination as sources of depression among U.S. women. Psychology of Women Quarterly, 27, 101-113.

Bhui, K., \& Fletcher, A. (2000). Common mood and anxiety states: gender differences in the protective effect of physical activity. Social psychiatry and psychiatric epidemiology, 35, 28-35.

Bhuiga, A., \& Chowdhury, M. (2002). Beneficial effects of a woman-focused development programme on child survival: Evidence from rural Bangladesh. Social Science and Medicine, 55, $1553-1560$.

Bijl, R. V., \& Ravelli, A. (2000). Current and residual functional disability associated with psychopathology: Findings from the Netherlands Mental Health Survey and Incidence Study (NEMESIS). Psychological Medicine, 30, 657-668.

Bijl, R. V., Ravelli, A., \& Van Zessen, G. (1998). Prevalence of psychiatric disorder in the general population: Results of the Netherlands Mental Health Survey and Incidence Study (NEMESIS). Social Psychiatry and Psychiatric Epidemiology, 33, 587-595.

Bond, L., Toumbourou, J.W., Thomas, L., Catalano, R.F., \& Patton, G. (2005). Individual, family, school and community risk and protective factors for depressive symptoms in adolescents: A comparison of risk profiles for substance use and depressive symptoms. Prevention Science, 6, 73-88.

Bosscher, R. J., \& Baardman, I. (1989). Het meten van gepercipieerde competentie bij psychiatrische patienten. [Measuring perceived competencies in psychiatric patients.] Bewegen en Hulpverlening, 6 , 312-322.

Bosscher, R. J., Smit, J. H., \& Kempen, G. I. J. M. (1997). Algemene competentieverwachtingen bij ouderen; Een onderzoek naar de psychometrische kenmerken van de Algemene Competentieschaal (ALCOS). [General competency expectations in elderly; A study into the psychometric properties of the general competency scale]. Nederlands Tijdschrift voor Psychology, 52.

Bosscher, R. J., Van Der, A. A. H., Van Dasler, M., Deeg, D. J., \& Smit, J. H. (1995). Physical performance and physical self-efficacy in the elderly. A pilot study. Journal of Aging and Health 7, 459-475.

Bouma, J., Ranchor, A. V., Sanderman, R., \& Van Sonderen, E. (1995). Het meten van symptomen van depressie met de CES-D: een handleiding. [Measuring depressive symptoms with the CES-D: A manual.] Groningen: Noordelijk Centrum voor Gezondheidsvraagstukken.

Bower, P., \& Gilbody, S. (2005). Stepped care in psychological therapies: Access, effectiveness and efficiency: Narrative literature review. British Journal of Psychiatry, 186, 11-17.

Bowling, A. (2005). Mode of questionnaire administration can have serious effects on data quality. Journal of Public Health, 27, 281-291.

Bridges, K. R., \& Sanderman, R. (2002). The Irrational Beliefs Inventory: cross cultural comparisons between American and Dutch samples. Journal of Rational-Emotive and Cognitive-Behavior Therapy, 20, 65-71.

Brosse, A. L., Sheets, E. S., Lett, H. S., \& Blumenthal, J. A. (2002). Exercise and the treatment of clinical depression in adults: Recent findings and future directions. Sports Medicine, 32, 741760 . 
Brown, C. H., \& Liao, J. (1999). Principles for designing randomized preventive trials in mental health: An emerging developmental epidemiology paradigm. American Journal of Community Psychology, 27, 673-710.

Brown, G. W., Bifulco, A. T., \& Harris, T. O. (1987). Life events, vulnerability and onset of depression: Some refinements. British Journal of Psychiatry, 150, 30-42.

Brown, G. W., Bifulco, A., Veiel, H. O., \& Andrews, B. (1990). Self-esteem and depression. II. Social correlates of self-esteem. Social Psychiatry and Psychiatric Epidemiology, 25, 225-234.

Brown, G. W., Harris, T. O., \& Hepworth, C. (1995). Loss, humiliation and entrapment among women developing depression: A patient and non-patient comparison. Psychological Medicine, 25, 7-21.

Brown, G.W., \& Moran, P.M. (1997). Single mothers, poverty and depression. Psychological Medicine, 27, 21-33.

Brown, R. A., \& Lewinsohn, P. M. (1984). A psychoeducational approach to the treatment of depression: Comparison of group, individual, and minimal contact procedures. Journal of Consulting and Clinical Psychology, 52, 774-783.

Brown, W. J., Ford, J. H., Burton, N. W., Marshall, A. L., \& Dobson, A. J. (2005). Prospective study of physical activity and depressive symptoms in middle-aged women. American Journal of Preventive Medicine, 29, 265-272.

Bullock, L. F., Wells, J. E., Duff, G. B., \& Hornblow, A. R. (1995). Telephone support for pregnant women: Outcome in late pregnancy. New Zealand Medical Journal, 108, 476-478.

Busch, M. C. M., \& Schrijvers, C. T. M. (2010). Effecten van leefstijlinterventies gericht op lagere sociaaleconomische groepen. [Effects of life-style interventions targeted at low socioeconomic groups.] Bilthoven: RIVM.

Cardemil, E. V., Kim, S., Pinedo, T. M., \& Miller, I. W. (2005). Developing a culturally appropriate depression prevention program: The family coping skills program. Cultural Diversity and Ethnic Minority Psychology, 11, 99-112.

Cassem, E.H. (1995). Depressive disorders in the medically ill. An overview. Psychosomatics, 36, S210.

CBS. (2008). Gemeente Op Maat 2006: Maastricht. [Community Measure 2006: Maastricht.] Voorburg/Heerlen: Centraal Bureau voor de Statistiek.

CBS. (2010). Standaard Onderwijsindeling 2006-Editie 2010/'11 [Standard Education Classification 2006- Edition 2010/2011]. Voorburg/Heerlen: Centraal Bureau voor de Statistiek.

Chan, K. S., Orlando, M., Ghosh-Dastidar, B., Duan, N., \& Sherbourne, C. D. (2004). The interview mode effect on the Center for Epidemiological Studies Depression (CES-D) Scale: An item response theory analysis. Medical Care, 42, 281-289.

Cheng, S.T., \& Chan, A.C.M. (2005). The Center for Epidemiological Studies Depression scale in older Chinese: Thresholds for long and short forms. International Journal of Geriatric Psychiatry, 20, 465-470.

Choenarom, C., Williams, R. A., \& Hagerty, B. M. (2005). The role of sense of belonging and social support on stress and depression in individuals with depression. Archives of Psychiatric Nursing, 19, 18-29.

Clarke, G. N., Hornbrook, M., Lynch, F., Polen, M., Gale, J., Beardslee, W., O'Connor, E., \& Seeley, J. (2001). A randomized trial of a group cognitive intervention for preventing depression in adolescent offspring of depressed parents. Archives of General Psychiatry, 58, 1127-1134.

Cohen, J. (1988). Statistical power analysis for the Behavioural Sciences (2nd ed.). New Jersey: Lawrence Erlbaum.

Cohen, S., \& Janicki-Deverts, D. (2010). Who's stressed? Distributions of psychological stress in the United States in probability samples from 1983, 2006 and 2009. Journal of Applied Social Psychology, in press.

Cohen, S., \& Williamson, G.M. (1987). Perceived stress in a probability sample of the United States. In S. Spacapan, \& S. Oskamp (Eds.), The social psychology of health (pp. 31-47). Newbury Park, California: SAGE Publications.

Cohen, S., Kamarck, T., \& Mermelstein, R. (1983). A global measure of perceived stress. Journal of Health and Social Behavior, 24, 385-396. 
Collins, R., Lee, R. E., Albright, C. L., \& King, A. C. (2004). Ready to be physically active? The effects of a course preparing low-income multiethnic women to be more physically active. Health Education and Behavior, 31, 47-64.

Conn, V. S. (2010). Depressive symptoms outcomes of physical activity interventions: Metaanalysis findings. Annals of Behavioral Medicine, 39, 128-138.

Cox, M. F., Van Marwijk, H. W. J., \& Boer, C. (2006). Wat heeft vijf jaar kwaliteitsbeleid ggz voor de huisarts opgeleverd? [What does five years of quality mental health care mean for the general practitioner?] Huisarts en Wetenschap, 49, 365-369.

Craft, L. L., Freund, K. M., Culpepper, L., \& Perna, F. M. (2007). Intervention study of exercise for depressive symptoms in women. Journal of Women's Health, 16, 1499-1509.

Crockett, K., Zlotnick, C., Davis, M., Payne, N., \& Washington, R. (2008). A depression preventive intervention for rural low-income African-American pregnant women at risk for postpartum depression. Archives of Women's Mental Health, 11, 319-325.

Cuijpers, P., \& Dekker, J. (2005). Psychologische behandeling van depressie: een systematisch overzicht van meta-analysen. [Psychological treatment of depression: A systematic review of metaanalyses.] Nederlands Tijdschrift voor Geneeskunde, 149, 1892-1897.

Cuijpers, P., \& Smit, F. (2004). Subthreshold depression as a risk indicator for major depressive disorder: A systematic review of prospective studies. Acta Psychiatrica Scandinavia, 109, 325331.

Cuijpers, P., De Graaf, R., \& Van Dorsselaer, S. (2004). Minor depression: Risk profiles, functional disability, health care use and risk of developing major depression. Journal of Affective Disorders, 79, 71-79.

Cuijpers, P., Munoz, R. F., Clarke, G. N., \& Lewinsohn, P. M. (2009). Psychoeducational treatment and prevention of depression: The "Coping with Depression" course thirty years later. Clinical Psychology Review, 29, 449-458.

Cuijpers, P., Smit, F., \& Van Straten, A. (2007). Psychological treatments of subthreshold depression: A meta-analytic review. Acta Psychiatrica Scandinavia, 115, 434-441.

Cuijpers, P., Van Straten, A., Warmerdam, L., \& Smits, N. (2008). Characteristics of effective psychological treatments of depression: A metaregression analysis. Psychotherapy Research, 18, 225-236

Cutrona, C.E., Wallace, G., \& Wesner, K.A. (2006). Neighborhood characteristics and depression: An examination of stress processes. Current Directions in Psychological Science, 15, 188-192.

Dalstra, J. A. A., Kunst, A. E., Geurts, J. J. M., Frenken, F. J. M., \& Mackenbach, J. P. (2002). Trends in socioeconomic health inequalitiets in the Netherlands, 1981-1999. Journal of Epidemiology and Community Health, 56, 927-934.

D'Angelo, E. J., Llerena-Ouinn, R., Shapiro, R., Colon, F., Rodriguez, P., Gallagher, K., \& Beardslee, W. R. (2009). Adaptation of the preventive intervention program for depression for use with predominantly low-income Latino families. Family Process, 48, 269-291.

De Graaf, R., Ten Have, M., \& Van Dorsselaer, S. (2010). De psychische gezondheid van de Nederlandse bevolking. NEMESIS-2: Opzet en eerste resultaten. [The mental health of the Dutch population. NEMESIS-2: Methods and first results]. Utrecht: Trimbos-instituut.

Demyttenaere, K., Bruffaerts, R., Posada-Villa, J., Gasquet, I., Kovess, V., Lepine, J. P., Angermeyer, M. C., Bernert, S., de Girolamo, G., Morosini, P., Polidori, G., Kikkawa, T., Kawakami, N., Ono, Y., Takeshima, T., Uda, H., Karam, E. G., Fayyad, J. A., Karam, A. N., Mneimneh, Z. N., Medina-Mora, M. E., Borges, G., Lara, C., de Graaf, R., Ormel, J., Gureje, O., Shen, Y., Huang, Y., Zhang, M., Alonso, J., Haro, J. M., Vilagut, G., Bromet, E. J., Gluzman, S., Webb, C., Kessler, R. C., Merikangas, K. R., Anthony, J. C., VonKorff, M. R., \& Wang, P. S. (2004). Prevalence, severity, and unmet need for treatment of mental disorders in the World Health Organization World Mental Health Surveys. JAMA, 291, 2581-2590.

Den Boer, P. C. A. M., Wiersma, D., Russo, S., \& Van den Bosch, R. J. (2005). Paraprofessionals for anxiety and depressive disorders. Cochrane Database of Systematic Reviews 2005, 18, CD004688.

Dennis, C. L. (2005). Psychosocial and psychological interventions for prevention of postnatal depression: Systematic review. British Medical Journal, 331, 15-18. 
Denton, M., Prus, S., \& Walters, V. (2004). Gender differences in health: A Canadian study of the psychosocial, structural and behavioral determinants of health. Social Science and Medicine, 58, 2585-2600.

Dohrenwend, B. P., Levav, I., Shrout, P. E., \& Schwartz, S. (1992). Socioeconomic status and psychiatric disorders. The causation-selection issue. Science, 255, 946-952.

Droomers, M., Schrijvers, C. T. M., Van de Mheen, H., \& Mackenbach, J. P. (1998). Educational differences in leisure-time physical inactivity: A descriptive and explanatory study. Social Science \& Medicine, 47, 1665-1676.

Dunn, A. L., Trivedi, M. H., Kampert, J. B., Clark, C. G., \& Chambliss, H. O. (2005). Exercise treatment for depression: Efficacy and dose response. American Journal of Preventive Medicine, 28, 1-8.

Eaton, W., \& Muntaner, C. (1999). Socioeconomic stratification and mental disorder. In A. V. Horwitz \& T. L. Scheid (Eds.), A handbook for the study of mental health: social contexts, theories and systems (pp. 259-283). New York: Cambridge University Press.

Egger, M., Davey Smith, G., Schneider, M., \& Minder, C. (1997). Bias in meta-analysis detected by a simple, graphical test. British Medical Journal, 315, 629-634.

Ekkekakis, P., Hall, E. E., \& Petruzzello, S. J. (2005). Variation and homogeneity in affective responses to physical activity of varying intensities: An alternative perspective on doseresponse based on evolutionary considerations. Journal of Sports Sciences, 23, 477-500.

Elliott, M. (2001). Gender differences in causes of depression. Women and Health, 33, 183-198.

Emmen, M. J., Meijer, S. A., Heideman, J. M. C., Laurant, M. G. H., \& Verhaak, P. F. M. (2007). Versterking van de eerstelijns GGZ: effecten op langere termijn. [Reinforcement of primary mental health care: Long-term effects.] TSG: Tijdschrift voor Gezondheidswetenschappen, 85, 166.

Ennis, N. E., Hobfoll, S. E., \& Schroder, K. E. (2000). Money doesn't talk, it swears: How economic stress and resistance resources impact inner-city women's depressive mood. American Journal of Community Psychology, 28, 149-173.

Falconnier, L., \& Elkin, I. (2008). Addressing economic stress in the treatment of depression. American Journal of Orthopsychiatry, 787, 37-46.

Faulkner, G. (2009). Promoting physical activity for mental health: A complex intervention? Mental Health and Physical Activity, 2, 1-3.

Fleischer, N.L., Fernald, L.C., \& Hubbard, A.E. (2007). Depressive symptoms in low-income women in rural Mexico. Epidemiology, 18, 678-685.

Fox, K. R. (2000). The effects of exercise on self-perceptions and self-esteem. In S. J. H. Biddle \& K. R. Fox \& S. H. Boutcher (Eds.), Physical activity and psychological well-being (pp. 88-117). London: Routledge.

Fremont, J., \& Craighead, L. W. (1987). Aerobic exercise and cognitive therapy in the treatment of dysphoric moods. Cognitive Therapy and Research, 11, 241-251.

Fryers, T., Melzer, D., \& Jenkins, R. (2003). Social inequalities and the common mental disorders. A systematic review of the evidence. Social Psychiatry and Psychiatric Epidemiology, 38, 229-237.

Gadalla, T. M. (2008). Comparison of users and non-users of mental health services among depressed women: A national study. Women and Health, 47, 1-19.

Gallo, L. C., \& Matthews, K. A. (2003). Understanding the association between socioeconomic status and physical health: Do negative emotions play a role? Psychological Bulletin, 129, 10-51.

Gallo, L. C., Bogart, L. M., Vranceanu, A. M., \& Matthews, K. A. (2005). Socioeconomic status, resources, psychological experiences, and emotional responses: A test of the reserve capacity model. Journal of Personality and Social Psychology, 88, 386-399.

Giles-Corti, B., \& Donovan, R. J. (2002). Socioeconomic status differences in recreational physical activity levels and real and perceived access to a supportive physical environment. Preventive Medicine, 35, 601-611.

Gilliss, C. L., Lee, K. A., Gutierrez, Y., Taylor, D., Beyene, Y., Neuhaus, J., \& Murrell, N. (2001). Recruitment and retention of healthy minority women into community-based longitudinal research. Journal of Women's Health and Gender-Based Medicine, 10, 77-85.

Girgus, J. S., \& Nolen-Hoeksema, S. (2006). Cognition and depression. In C. L. M. Keyes \& S. H. Goodman (Eds.), Women and depression: a handbook for the social, behavioral and biomedical sciences (pp. 147-175). Cambridge: University Press. 
Glasgow, R. E., Vogt, T. M., \& Boles, S. M. (1999). Evaluating the public health impact of health promotion interventions: The RE-AIM framework. American Journal of Public Health, 89, 13221327.

Gleser, L. J., \& Olkin, I. (1994). Stochastically dependent effect sizes. In H. Cooper \& L. V. Hedges (Eds.), The Handbook of Research Synthesis (pp. 339-355). New York: Russell Sage Foundation.

Goodwin, R. D. (2003). Association between physical activity and mental disorders among adults in the United States. Preventive Medicine, 36, 698-703.

Gordon, R. S. (1983). An operational classification of disease prevention. Public Health Reports, 98, 107-109.

Grant, K. E., Lyons, A. L., Finkelstein, J. A. S., Conway, K. M., Reynolds, L. K., O'Koon, J. H., Waitkoff, G. R., \& Hixcks, K. J. (2004). Gender differences in rates of depressive symptoms among low-income, urban, African American youth: A test of two mediational hypotheses. Journal of Youth and Adolescence, 33, 523-533.

Green, B. L., \& Rodgers, A. (2001). Determinants of social support among low-income mothers: A longitudinal analysis. American Journal of Community Psychology, 29, 419-441.

Greenberg, M. T., Domitrovich, C., \& Bumbarger, B. (2001). The prevention of mental disorders in school-aged children: Current state of the field. Prevention and Treatment, 4, No pagination specified.

Groen, G., Pössel, P., Al-Wiswasi, S., \& Petermann, F. (2003). Universelle, schulbasierte prävention der depression im jugendalter: Ergebnisse einer follow-up studie [Universal, schoolbased prevention of depression in youngsters: Results of a follow-up study.] Kindheit und Entwicklung, 12, 164-174.

Groh, C.J. (2007). Poverty, mental health and women: Implications for psychiatric nurses in primary care settings. Journal of the American Psychiatric Nurses Association, 13, 267-274.

Grote, N. K., Zuckoff, A., Swartz, H., Bledsoe, S. E., \& Geibel, S. (2007). Engaging women who are depressed and economically disadvantaged in mental health treatment. Social Work, 52, 295308.

Grzywacz, J.G., Almeida, D.M., Neupert, S.D., \& Ettner, S.L. (2004). Socioeconomic status and health: A micro-level analysis of exposure and vulnerability to daily stressors. Journal of Health and Social Behavior, 45, 1-16.

Gutierrez-Lobo, K., Wölfl, G., Scherer, M., Anderer, P., \& Schmidl-Mohl, B. (2000). The gender gap in depression reconsidered: The influence of marital and employment status on the female/male ratio of treated incidence rates. Social Psychiatry and Psychiatric Epidemiology, 35, 202-210.

Hajema, K. J. (2007). Epidemiologische gegevens voor lokaal gezondheidsbeleid in Zuid-Limburg [Epidemiological data for local health policy in the Southern-Limburg region]. Geleen: GGD Zuid Limburg.

Hamad, R., Fernald, L.C.H., Karlan, D.S., \& Zinman, J. (2008). Social and economic correlates of depressive symptoms and perceived stress in South African adults. Journal of Epidemiological and Community Health, 62, 538-544.

Hammen, C. (1991). Generation of stress in the course of unipolar depression. Journal of Abnormal Psychology, 100, 555-561.

Hammen, C. (2003). Interpersonal stress and depression in women. Journal of Affective Disorders, 74, 49-57.

Hammen, C. (2006). Stress generation in depression: Reflections on origins, research, and future directions. Journal of Clinical Psychology.

Hammen, C., Kim, E. Y., Eberhart, N. K., \& Brennan, P. A. (2009). Chronic and acute stress and the prediction of major depression in women. Depression and Anxiety, 26, 718-723.

Harachi, T. W., Abbott, R. D., Catalano, R. F., Haggerty, K. P., \& Fleming, C. B. (1999). Opening the black box: Using process evaluation measures to assess implementation and theory building. American Journal of Community Psychology, 27, 711-731.

Haringsma, R., Engels, G. I., Cuijpers, P., \& Spinhoven, P. (2005). Effectiveness of the Coping With Depression (CWD) course for older adults provided by the community-based mental health care system in the Netherlands: A randomized controlled field trial. International Psychogeriatrics, 28, 1-19. 
Hassmén, P., Koivula, N., \& Uutela, A. (2000). Physical exercise and psychological well-being: A population study in Finland. Preventive Medicine, 30, 17-25.

Hauenstein, E. J. (1996). Testing innovative nursing care: Home intervention with depressed rural women. Issues in Mental Health Nursing, 17, 33-50.

Hauenstein, E. J. (1996a). A nursing practice paradigm for depressed rural women: Theoretical basis. Archives of Psychiatric Nursing, 10, 283-292.

Hayes, A.F. (2009). Beyond Baron and Kenny: Statistical mediation analysis in the new millennium. Communication Monographs, 76, 408-420.

Hedges, L. V., \& Olkin, I. (1985). Statistical methods for meta-analysis. San Diego, CA: Academic Press.

Henderson, C., Diez Roux, A.V., Jacobs, D.R., Kiefe, C.I., West, D., \& Williams, D.R. (2005). Neighborhood characteristics, individual level socioeconomic factors, and depressive symptoms in young adults: The CARDIA study. Journal of Epidemiological and Community Health, 59, 322-328.

Heneghan, A.M., Johnson Silver, E., Bauman, L.J., Westbrook, L.E., \& Stein, R.E.K. (1998). Depressive symptoms in inner-city mothers of young children: Who is at risk? Pediatrics, 102, 1394-1400.

Higgins, J. P., Thompson, S. G., Deeks, J. J., \& Altman, D. G. (2003). Measuring inconsistency in meta-analyses. British Medical Journal, 327, 557-560.

Hill, C. V., Oei, T. R., \& Hill, M. A. (1989). An empirical investigation of the specificity and sensitivity of the Automatic Thoughts Questionnaire and Dysfunctional Attitudes Scale. Journal of Psychopathology and Behavioral Assessment, 11, 291-311.

Hobfoll, S. E., Johnson, R. J., Ennis, N., \& Jackson, A. P. (2003). Resource loss, resource gain, and emotional outcomes among inner city women. Journal of personality and social psychology 84 , 632-643.

Hohmann, A. A., \& Shear, M. K. (2002). Community-based intervention research: Coping with the "noise" of real life in study design. American Journal of Psychiatry, 159, 201-207.

Hollon, S. D., \& Kendall, R. C. (1980). Cognitive self-statements in depression: Development of an automatic thoughts questionnaire. Cognitive Therapy and Research, 4, 383-395.

Hollon, S. D., Munoz, R. F., Barlow, D. H., Beardslee, W. R., Bell, C. C., Bernal, G., Clarke, G. N., Franciosi, L. P., Kazdin, A. E., Kohn, L., Lineham, M. M., Markowitz, J. C., Miklowitz, D. J., Persons, J. B., Niederehe, G., \& Sommers, D. (2002). Psychosocial intervention development for the prevention and treatment of depression: Promoting innovation and increasing access. Biological Psychiatry, 52, 610-630.

Horowitz, J. L., \& Garber, J. (2006). The prevention of depressive symptoms in children and adolescents: A meta-analytic review. Journal of Consulting and Clinical Psychology, 74, 401-415.

House, J. S., Lepkowski, J. M., Kinney, A. M., Mero, R. P., Kessler, R. C., \& Herzog, A. R. (1994) The social stratification of aging and health. Journal of Health and Social Behavior, 35, 213-234.

Hudson, D. B., Campbell-Grossman, C., Keating-Lefler, R., \& Cline, P. (2008). New mothers network: The development of an internet-based social support intervention for African American mothers. Issues in Comprehensive Pediatric Nursing, 31, 23-25.

Huurre, T., Eerola, M., Rahkonen, O., \& Aro, H. (2007). Does social support affect the relationship between socioeconomic status and depression? A longitudinal study from adolescence to adulthood. Journal of Affective Disorders, 100, 55-64.

Ilgen, M.A., \& Hutchison, K.E. (2005). A history of major depressive disorder and the response to stress. Journal of Affective Disorders, 86, 143-150.

Ireys, H. T., DeVet, K. A., \& Chernoff, R. (2001). Who joins a preventive intervention? How risk status predicts enrolment. Journal of Community Psychology, 29, 417-427.

Jackson, J.S., Knight, K.M., \& Rafferty, J.A. (2010). Race and unhealthy behaviors: Chronic stress, the HPA axis, and physical and mental health disparities over the life course. American Journal of Public Health, 100, 933-939.

Jané- Llopis, E., Hosman, C., Jenkins, R., \& Anderson, P. (2003). Predictors of efficacy in depression prevention programmes. British Journal of Psychiatry, 183, 384-397. 
Jesse, D. E., Dolbier, C. L., \& Blanchard, A. (2008). Barriers to seeking help and treatment suggestions for prenatal depressive symptoms: Focus groups with rural low-income women. Issues in Mental Health Nursing, 29, 3-19.

Judd, L. L., Schettler, P. J., \& Akiskal, H. S. (2002). The prevalence, clinical relevance, and public health significance of subthreshold depressions. Psychiatric Clinics of North America, 25, 685698.

Kahn, R.S., Wise, P.H., Kennedy, B.P., \& Kawachi, I. (2000). State income inequality, household income, and maternal mental and physical health: Cross sectional national survey. British Medical Journal, 321, 1311-1315.

Kalil, A., Born, C. E., Kunz, J., \& Caudill, P. J. (2001). Life stressors, social support, and depressive symptoms among first-time welfare recipients. American Journal of Community Psychology, 29, 355-369.

Kamphuis, C. B., Van Lenthe, F. J., Giskes, K., Huisman, M., Brug, J., \& Mackenbach, J. P. (2009). Socioeconomic differences in lack of recreational walking among older adults: The role of neighbourhood and individual factors. International Journal of Behavioral Nutrition and Physical Activity, 6, doi:10.1186/1479-5868-1186-1181.

Katerndahl, D. A., \& Parchman, M. (2002). The ability of the stress process model to explain mental health outcomes. Comprehensive Psychiatry, 34, 351-360.

Katon, W. J., \& Ludman, E. J. (2003). Improving services for women with depression in primary care settings. Psychology of Women Quarterly, 27, 114-120.

Kazdin, A. E. (2001). Progression of therapy research and clinical application of treatment require better understanding of the change process. Clinical Psychology: Science and Practice, 8, 143-151.

Kazdin, A. E., \& Nock, M. K. (2003). Delineating mechanisms of change in child and adolescent therapy: Methodological issues and research recommendations. Journal of Child Psychology and Psychiatry, 44, 1116-1129.

Kessler, R. C., Zhao, S., Blazer, D. G., \& Swartz, M. (1997). Prevalence, correlates, and course of minor depression and major depression in the national comorbidity survey. Journal of Affective Disorders, 45, 19-30.

Khan, K. S., Daya, S., \& Jadad, A. (1996). The importance of quality of primary studies in producing unbiased systematic reviews. Archives of Internal Medicine, 156, 661-666.

Kickbusch, I., McCann, W., \& Sherbon, T. (2008). Adelaide revisited: From healthy public policy to Health in All Policies. Health Promot Int, 23, 1-4.

Kickbush, I. (2009). Policy Innovation for Health. Geneve: Springer Science+Business Media.

Klose, M., \& Jacobi, F. (2004). Can gender differences in the prevalence of mental disorders be explained by sociodemographic factors? Archives of Women's Mental Health, 7, 133-148.

Kohn, L. P., Oden, T., Muñoz, R. F., Robinson, A., \& Leavitt, D. (2002). Adapted cognitive behavioral group therapy for depressed low- income African American women. Community Mental Health Journal, 38, 497-504.

Koopmans, P. C., Sanderman, R., Timmerman, I., \& Emmelkamp, P. M. G. (1994). The Irrational Beliefs Inventory (IBI): Development and psychometric evaluation. European Journal of Psychological Assessment, 10, 15-27.

Krieger, N., Williams, D. R., \& Moss, N. E. (1997). Measuring social class in US public health research: Concepts, methodologies, and guidelines. Annual Review of Public Health, 18, 341378.

Kuehner, C. (2003). Gender differences in unipolar depression: an update of epidemiological findings and positive explanations. Acta Psychiatrica Scandinavica, 108, 163-174.

Lanen, W., Gelissen, T., Ebben, N., \& van der Waerden, J. (2008). Draaiboek Bewegen zonder zorgen [Course manual Exercise without Worries]. Maastricht

Laperriere, A., Ironson, G. H., Antoni, M. H., Pomm, H., Jones, D., Ishii, M., Lydston, D., Lawrence, P., Grossman, A., Brondolo, E., Cassells, A., Tobin, J. N., Schneiderman, N., \& Weiss, S. M. (2005). Decreased depression up to one year following CBSM+ intervention in depressed women with AIDS: The Smart/EST women's project. Journal of Health Psychology, 10, 223-231.

Latkin, C. A., \& Curry, A. D. (2003). Stressful neighborhoods and depression: A prospective study of the impact of neighborhood disorder. Journal of Health and Social Behavior, 44, 34-44. 
Lara, M. A., Navarro, C., Rubi, N. A., \& Mondragon, L. (2003a). Outcome results of two levels of intervention in low- income women with depressive symptoms. American Journal of Orthopsychiatry, 73, 35-43.

Lara, M. A., Navarro, C., Navarrete, L., Mondragon, L., \& Rubi, N. A. (2003b). Segumimiento a dos anos de una intervencion psicoeducativa para mujeres con sintomas de depresion, en servicios de salud para poblacion abierta. [Two-year follow-up of a psycho-educational intervention for women with depressive symptoms in health services for the general population.] Salud Mental, 26, 27-36.

Laurant, M. G. H. (2001). Geestelijke gezondheidszorg bij de huisarts. [Mental health care in general practice.] Informatiebrief Nederlands Huisartsen Genootschap, 56.

Lawlor, D. A., \& Hopker, S. W. (2001). The effectiveness of exercise as an intervention in the management of depression: Systematic review and metaregression analysis of randomised controlled trials. British Medical Journal, 322, 1-8.

Le, H., Lara, M. A., \& Perry, D. F. (2008). Recruiting Latino women in the U.S. and women in Mexico in postpartum depression prevention research. Archives of Women's Mental Health, 11, 159-169.

Levy, L. B., \& O'Hara, M. W. (2010). Psychotherapeutic interventions for depressed, low-income women: A review of the literature. Clinical Psychology Review, 30, 934-950.

Lewinsohn, R. M., \& Clarke, G. N. (1984). Group treatment of depressed individuals: The "Coping with Depression" course. Advances in Behaviour Research and Therapy, 6, 99-114.

Link, B. G., \& Phelan, J. C. (1995). Social conditions as fundamental causes of disease. Journal of Health and Social Behavior, 35, 80-94.

Lipman, E. L., \& Boyle, M. H. (2005). Social support and education groups for single mothers: A randomized controlled trial of a community based program. Canadian Medical Association Journal, 173, 1451-1456.

Lipsey, M. W., \& Wilson, D. B. (2001). Practical Meta-analysis. Thousand Oaks: Sage.

Liu, R.T., \& Alloy, L.B. (2010). Stress generation in depression: A systematic review of the empirical literature and recommendations for future study. Clinical Psychology Review, 30, 582-593

Lorant, V., Deliege, D., Eaton, W., Robert, A., Philippot, P., \& Ansseau, M. (2003). Socioeconomic inequalities in depression: A meta- analysis. American Journal of Epidemiology, 157, 98-112.

Lowther, M., Mutrie, N., \& Scott, E. M. (2002). Promoting physical activity in a socially and economically deprived community: A 12 month randomized control trial of fitness assessment and exercise consultation. Journal of Sports Sciences, 20, 577-588.

Lundberg, I., Damström Thakker, K., Hällström, T., \& Forsell, Y. (2005). Determinants of nonparticipation and the effects of non-participation on potential cause-effect relationships, in the PART study on mental disorders. Social Psychiatry and Psychiatric Epidemiology, 40, 475-483.

Luteijn, F., Starren, J., \& van Dijk, H. (1985). Handleiding bij de Nederlandse Persoonlijkheidsvragenlijst $N P V$. [Manual of the Dutch Personality Inventory NPV]. Lisse: Swets en Zeitlinger.

Mackenbach, J. P., \& Bakker, M. J. (2003). Tackling socioeconomic inequalities in health: Analysis of European experiences. Lancet, 362, 1409-1414.

Mackenbach, J. P., Kunst, A. E., Cavelaars, A. E., Groenhof, F., \& Geurts, J. J. (1997). Socioeconomic inequalities in morbidity and mortality in western Europe. The EU Working Group on Socioeconomic Inequalities in Health. Lancet, 349, 1655-1659.

Mair, C., Diez Roux, A.V., \& Galea, S. (2008). Are neighbourhood characteristics associated with depressive symptoms? A review of evidence. Journal of Epidemiology and Community Health, $62,940-946$.

Manber, R., Allen, J. J. B., \& Morris, M. M. (2002). Alternative treatments for depression: Empirical support and relevance to women. Journal of Clinical Psychiatry, 63, 628-640.

Martinsen, E. W. (2008). Physical activity in the prevention and treatment of anxiety and depression. Nordic Journal of Psychiatry, 62.

Matheson, F.I., Moineddin, R., Dunn, J.R., Creatore, M.I., Gozdyra, P., \& Glazier, R.H. (2006). Urban neighborhoods, chronic stress, gender and depression. Social Science and Medicine, 63, 2604-2626.

McConagle, K. A., \& Kessler, R. C. (1990). Chronic stress, acute stress, and depressive symptoms. American Journal of Community Psychology, 18, 681-706. 
McDonough, P., \& Walters, V. (2001). Gender and health: Reassessing patterns and explanations. Social Science and Medicine, 52, 547-559.

McGale, N., McArdle, S., \& Gaffney, P. (2010). Exploring the effectiveness of an integrated exercise/CBT intervention for young men's mental health. British Journal of Health Psychology, DOI:10.1348/135910710X522734.

McKee, M. D., Zayas, L. H., Fletcher, J., Rhonda, C. B., \& Nam, S. H. (2006). Results of an intervention to reduce perinatal depression among low-income minority women in community primary care. Journal of Social Service Research, 32, 63-81.

McLaren, L., McIntyre, L., \& Kirkpatrick, S. (2010). Rose's population strategy of prevention need not increase social inequalities in health. International Journal of Epidemiology, 39, 372-377.

McLeod, J. D., \& Nonnemaker, J. M. (1999). Social stratification and inequality. In C. S. Aneshensel \& J. C. Phelan (Eds.), Handbook of the sociology of mental health (pp. 321-344). New York: Kluwer Academic/Plenum Publishers.

McLeod, J., Johnston, J., \& Griffin, J. (2000). A naturalistic study of the effectiveness of time-limited counselling with low-income clients. European Journal of Psychotherapy, Counselling and Health, 3, 263-277.

Meeuwissen, J. A. C., Van der Feltz-Cornelis, C. M., Van Marwijk, H. W. J., Rijnders, P. B. M., \& Donker, M. C. H. (2008). A stepped care programma for depression management: An uncontrolled pre-post study in primary and secondary care in The Netherlands. International Journal of Integrated Care, 8, 1-11.

Meijer, S. A., Smit, F., Schoemaker, C., \& Cuijpers, P. (2006). Gezond verstand: evidence-based preventie van psychische stoornissen [Common sense: Evidence-based prevention of psychiatric disorders]. RIVM Rapport 270672001; VTV Themarapport. Bilthoven/Utrecht: RIVM/Trimbosinstituut.

Melchior, M., Chastang, J. F., Leclerc, A., Ribet, C., \& Rouillon, F. (2010). Low socioeconomic position and depression persistence: Longitudinal results from the GAZEL cohort study. Psychiatry Research, doi:10.1016/j.psychres.2009.08.002.

Mendelson, T., \& Muñoz, R. F. (2006). Prevention of depression in women. In C. L. M. Keyes \& S. H. Goodman (Eds.), Women and depression: A handbook for the social, behavioral, and biomedical sciences (pp. 450-478). New York: Cambridge University Press.

Mendelson, T., Kubzansky, L. D., Datta, G. D., \& Buka, S. L. (2008). Relation of female gender and low socioeconomic status to internalizing symptoms among adolescents: A case of double jeopardy? Social Science E Medicine, 66, 1284-1296.

Merom, D., Phongsavan, P., Wagner, R., Chey, T., Marnane, C., Steel, Z., Silove, D., \& Bauman, A. (2008). Promoting walking as an adjunct intervention to group cognitive behavioral therapy for anxiety disorders-A pilot group randomized trial. Journal of Anxiety Disorders, 22, 959968.

Mezuk, B., Rafferty, J.A., Kershaw, K.N., Hudson, D., Abdou, C.M., Lee, H., Eaton, W.W., \& Jackson, J.S. (2010). Reconsidering the role of social disadvantage in physical and mental health: Stressful life events, health behaviors, race, and depression. American Journal of Epidemiology, DOI: 10.1093/aje/kwq283.

Michalsen, A., Grossman, P., Acil, A., Langhorst, J., Lüdtke, R., Esch, T., Stefano, G. B., \& Dobos, G. J. (2005). Rapid stress reduction and anxiolysis among distressed women as a consequence of a three-month intensive yoga program. Medical Science Monitor, 11, 555-561.

Mickelson, K. D., \& Kubzansky, L. D. (2003). Social distribution of social support: The mediating role of life events. American Journal of Community Psychology, 32, 265-281.

Miech, R. A., \& Hauser, R. M. (2001). Socioeconomic status and health at midlife: A comparison of educational attainment with occupation-based indicators. Annals of Epidemiology, 11, 75-84

Miranda, J., \& Green, B. L. (1999). The need for mental health services research focusing on poor young women. The Journal of Mental Health Policy and Economics, 2, 73-80.

Miranda, J., Chung, J. Y., Green, B. L., Krupnick, J., Siddique, J., Revicki, D. A., \& Belin, T. (2003). Treating depression in predominantly low-income young minority women: A randomized controlled trial. JAMA, 290, 57-65. 
Miranda, J., Green, B. L., Krupnick, J. L., Chung, J., Siddique, J., Belin, T., \& Revicki, D. (2006). Oneyear outcomes of a randomized clinical trial treating depression in low- income minority women. Journal of Consulting and Clinical Psychology, 74, 99-111.

Moher, D., Pham, B., Jones, A., Cook, D. J., Jadad, A. R., Moher, M., Tugwell, P., \& Klassen, T. P. (1998). Does quality of reports of randomized trials affect estimates of intervention efficacy reported in meta-analyses? Lancet, 352, 609-613.

Mojtabai, R. (2005). Trends in contacts with mental health professionals and cost barriers to mental health care among adults with significant psychological distress in the United States: 19972002. American Journal of Public Health, 95, 2009-2014.

Mol, G. D., Van de Lisdonk, E. H., Smits, J. P. J. M., Van den Hoogen, J. M. P., Bor, J. H. J., \& Westert, G. P. (2005). A widening health gap in general practice? Socioeconomic differences in morbidity between 1975 and 2000 in the Netherlands. Public Health, 119, 616-625.

Moons, K. G. M., Donders, A. R. T., Stijnen, T., \& Harrell, F. R. J. (2006). Using the outcome for imputation of missing predictor values was preferred. Journal of Clinical Epidemiology, 59, 1092-1101.

Mrazek, P. J., \& Haggerty, R. J. (1994). New directions in definitions. In P. J. Mrazek \& R. J. Haggerty (Eds.), Reducing risks for mental disorders: Frontiers for preventive intervention research (pp. 19-29). Washington: National Academy Press.

Mulvaney, C., \& Kendrick, D. (2005). Depressive symptoms in mothers of pre-school children: Effects of deprivation, social support, stress and neighborhood social capital. Social Psychiatry and Psychiatric Epidemiology, 40, 202-208.

Muñoz, R. F., \& Ying, Y. (1993). The prevention of depression: Research and practice. Baltimore: John Hopkins University Press.

Muñoz, R. F., Cuijpers, P., Smit, F., Barrera, A. Z., \& Leykin, Y. (2010). Prevention of major depression. Annual Review of Clinical Psychology, 6, 181-212.

Muñoz, R. F., Huynh-Nhu, L., \& Ghosh Ippen, C. (2000). We should screen for major depression. Applied and Preventive Psychology, 9, 123-133.

Muñoz, R. F., Le, H. N., Ippen, C. G., Ciaz, M. A., Urizar, G. G., Soto, J., Mendelson, T., Delucchi, K., \& Lieberman, A. F. (2007). Prevention of postpartum depression in low-income women: Development of the Mamás y Bebés/Mothers and Babies course. Cognitive and Behavioral Practice, 14, 70-83.

Muntaner, C., Eaton, W. W., Miech, R., \& OCampo, P. (2004). Socioeconomic position and major mental disorders. Epidemiologic Reviews, 26, 53-62.

Nadeem, E., Lange, J. M., \& Miranda, J. (2008). Mental health care preferences among low-income and minority women. Archives of Women's Mental Health, 11, 93-102.

Nakhaie, R., Lin, X., \& Guan, J. (2009). Social capital and the myth of minority self-employment: Evidence from Canada. Journal of Ethnic and Migration Studies, 35, 625-644.

Nation, M., Crusto, C., Wandersman, A., Kumpfer, K. L., Seybolt, D., Morrisey-Kane, E., \& Davino, K. (2003). What works in prevention: Principles of effective prevention programs. American Psychologist, 58, 449-456.

Nazroo, J. Y. (2005). Exploring gender difference in depression. Psychiatric Times, 18.

Nolen-Hoeksema, S., Larson, J., \& Grayson, C. (1999). Explaining the gender difference in depressive symptoms. Journal of Personality and Social Psychology, 77, 1061-1072.

Noordman, J. (2007). Weinig aandacht voor spanningen: Huisarts pikt psychosociale signalen vaak niet op. [Little attention for stress: GP does often not recognize psychosocial problems.] Medisch Contact, 62, 1994-1996.

O'Connell, M. E., Boat, T., \& Warner, K. E. (2009). Defining the scope of prevention. In M. E. O'Connell \& T. Boat \& K. E. Warner (Eds.), Preventing mental, emotional, and behavioral disorders among young people: Progress and possibilities. (pp. 59-69). Washington, DC: The National Academies Press.

Olson, R., Verley, J., Santos, L., \& Salas, C. (2004). What we teach students about the Hawthorne studies: A review of content within a sample of introductory I-O and OB textbooks. The Industrial-Organizational Psychologist, 41, 23-39.

Paluska, S. A., \& Schwenk, T. L. (2000). Physical activity and mental health: Current concepts. Sports Medicine, 29, 167-180. 
Pearlin, L. I. (1989). The sociological study of stress. Journal of Health and Social Behavior, 30, 241-256.

Pearlin, L. I. (1999a). The stress process revisited: Reflections on concepts and their interrelationships. In C. S. Aneshensel \& J. C. Phelan (Eds.), Handbook of the Sociology of Mental Health (pp. 395-415). New York: Kluwer Academic/Plenum Publishers.

Pearlin, L. I. (1999b). Stress and mental health: A conceptual overview. In A. V. Horwitz \& T. L. Scheid (Eds.), A handbook for the study of mental health. Social contexts, theories and systems (pp. 161-175). Cambridge: University Press.

Pearlin, L. I., Menaghan, E. G., Lieberman, M. A., \& Mullan, J. T. (1981). The stress process. Journal of Health and Social Behavior, 22, 337-356.

Pearlin, L.I. \& Schooler, C. (1978). The structure of coping. Journal of Health and Social Behavior, 19, 2-21.

Peden, A. R., Rayens, M. K., Hall, L. A., \& Grant, E. (2004). Negative thinking and the mental health of low- income single mothers. Journal of Nursing Scholarship, 36, 337-344.

Peden, A. R., Rayens, M. K., \& Hall, L. A. (2005). A community-based depression prevention intervention with low-income single mothers. Journal of the American Psychiatric Nurses Association, 11, 18-25.

Peden, A. R., Rayens, M. K., Hall, L. A., \& Grant, E. (2005). Testing an intervention to reduce negative thinking, depressive symptoms, and chronic stressors in low- income single mothers. Journal of Nursing Scholarship 37, 268-274.

Peluso, M. A. M., \& de Andrade, L. H. S. G. (2005). Physical activity and mental health: The association between exercise and mood. Clinics, 60, 61-70.

Penedo, F. J., \& Dahn, J. R. (2005). Exercise and well-being: A review of mental and physical health benefits associated with physical activity. Current Opinion in Psychiatry, 18, 189-193.

Perry, C. K., Rosenfeld, A. G., \& Kendall, J. (2007). Rural women walking for health. Western Journal of Nursing Research.

Phelan, J. C., Link, B. G., \& Tehranifar, P. (2010). Social conditions as fundamental causes of health inequalities: Theory, evidence and policy implications. Journal of Health and Social Behavior, 51, S28.

Picavet, H. S. J. (2001). National health surveys by mail or home interview: Effects on response. Journal of Epidemiological and Community Health, 55, 408-413.

Piccinelli, M., \& Wilkinson, G. (2000). Gender differences in depression: Critical review. British Journal of Psychiatry, 177, 486-492.

Podorefsky, D. L., McDonald- Dowdell, M., \& Beardslee, W. R. (2001). Adaptation of preventive interventions for a low-income, culturally diverse community. Journal of the American Academy for Child and Adolescent Psychiatry, 40, 879-886. .

Post, N. A. M., Zwakhals, S. L. N., \& Polder, J. J. (2010). Maatschappelijke baten. [Societal benefits.] Houten: RIVM.

Posternak, M. A., \& Miller. (2001). Untreated short- term course of major depression: A metaanalysis of outcomes from studies using wait- list control groups. Journal of Affective Disorders, $66,139-146$.

Preacher, K.J., \& Hayes, A.F. (2008). Asymptotic and resampling strategies for assessing and comparing indirect effects in multiple mediator models. Behavior Research Methods, 40, 879891.

Programmacommissie SEGV-II. (2001). Sociaal-economische gezondheidsverschillen verkleinen: Eindrapportage en beleidsaanbevelingen van de Programmacommissie SEGV-II. [Reducing socioeconomic inequalities in health. Final report and policy recommendations of the Programme Committee SEIH-II]. Den Haag: ZorgOnderzoek Nederland.

Radloff, L. S. (1977). The CES-D scale: A self-reportdepression scale for research in the general population. Applied Psychological Measurement 1, 385-401.

Raingruber, B. (2002). Client and provider perspectives regarding the stigma of and nonstigmatizing interventions for depression. Archives of Psychiatric Nursing, 16, 201-207.

Raudenbush, S. W. (1994). Random effects models. In H. Cooper \& L. V. Hedges (Eds.), The Handbook of Research Synthesis (pp. 301-321). New York: Russell Sage Foundation.

Regeringsverklaring. (2007). Van aandachtswijken tot krachtwijken [From problem districs to power districts]. Den Haag. 
Regidor, E. (2006). Social determinants of health: A veil that hides socioeconomic position and its relation with health. Journal of Epidemiology and Community Health, 60, 896-901.

Reid, J. (2004). Choosing health? A consultation on action to improve people's health. London: Department of Health.

Rochon, J. (1999). Issues in adjusting for covariates arising postrandomization in clinical trials. Drug Information Journal, 33, 1219-1228.

Roman, L. A., Gardiner, J. C., Lindsay, J. K., Moore, J. S., Luo, Z., Baer, L. J., Goddeeris, J. H., Shoemaker, A. L., Barton, L. R., Fitzgerald, H. E., \& Paneth, N. (2009). Alleviating perinatal depressive symptoms and stress: A nurse-community health worker randomized trial. Archives of Womens Mental Health, 12, 379-391.

Rose, G. (1985). Sick individuals and sick populations International Journal of Epidemiology, 14, 32-38 Reprinted in International Journal of Epidemiology, 2001, 2030:2427-2032.

Rose, G. (1992). The strategy of preventive medicine. Oxford: Oxford University Press.

Ross, C.E., \& Mirowsky, J. (2006). Sex differences in the effect of education on depression: Resource multiplication of resource substitution? Social Science and Medicine, 63, 1400-1413.

Salmon, P. (2001). Effects of physical exercise on anxiety, depression, and sensitivity to stress: A unifying theory. Clinical Psychology Review, 21, 33-61.

Santor, D. A., Zuroff, D. C., Ramsay, J. O., Cervantes, P., \& Palacios, J. (1995). Examining scale discriminability in the BDI and CES-D as a function of depressive severity. Psychological Assessment, 7, 131-139.

Sareen, J., Jagdeo, A., Cox, B. J., Clara, I., Ten Have, M., Belik, S. L., De Graaf, R., \& Stein, M. B. (2007). Perceived barriers to mental health service utilization in the United States, Ontario, and the Netherlands. Psychiatric Services, 58, 357-364.

Saunders, R. P., Evans, M. H., \& Joshi, P. (2005). Developing a process-evaluation plan for assessing health promotion program implementation: A how-to guide. Health Promotion Practice, 6, $134-$ 147.

Schaeffer, N. C., \& Presser, S. (2003). The science of asking questions. Annual Review of Sociology, 29, 65-88.

Schulz, A.J., Israel, B.A., Zenk, S.N., Parker, E.A., Lichtenstein, R., Shellman-Weir, S., \& Klem, A.B.L. (2006). Psychosocial stress and social support as mediators of relationships between income, length of residence and depressive symptoms among African American women on Detroit's eastside. Social Science and Medicine, 62, 510-522.

Scully, D., Kremer, J., Meade, M. M., Graham, R., \& Dudgeon, K. (1998). Physical exercise and psychological well being: A critical review. British Journal of Sports Medicine, 32, 111-120.

Seedat, S., Scott, K. M., Angermeyer, M. C., Berglund, P., Bromet, E. J., Brugha, T. S., Demyttenaere, K., de Girolamo, G., Haro, J. M., Jin, R., Karam, E. G., Kovess-Masfety, V., Levinson, D., Medina Mora, M. E., Ono, Y., Ormel, J., Pennell, B.-E., Posada-Villa, J., Sampson, N. A., Williams, D., \& Kessler, R. C. (2009). Cross-national associations between gender and mental disorders in the World Health Organization World Mental Health Surveys. Archives of General Psychiatry, 66, 785-795.

Seeman, T. E. (1991). Personal control and coronary artery disease: How generalized expectancies about control may influence disease risk. Journal of Psychosomatic Research, 35, 661-669.

Senturia, Y. D., McNiff Mortimer, K., Baker, D., Gergen, P., Mitchell, H., Joseph, C., \& Wedner, H. J. (1998). Successful techniques for retention of study participants in an inner-city population. Controlled Clinical Trials, 19, 544-554.

Sherer, M., Maddux, J. E., Mercandante, B., Prentice-Dunn, S., Jacobs, B., \& Rogers, R. W. (1982). The Self-efficacy Scale: Construction and validation. Psychological Reports, 51, 663-671.

Shih, J. H., \& Eberhart, N. K. (2007). Understanding the impact of prior depression on stress generation: Examining the roles of current depressive symptoms and interpersonal behaviours. British Journal of Psychology, 99, 413-426.

Shrout, P.E., \& Bolger, N. (2002). Mediation in experimental and nonexperimental studies: New procedures and recommendations. Psychological Methods, 7, 422-445.

Silver, E., Mulvey, E. P., \& Swanson, J. W. (2002). Neighborhood structural characteristics and mental disorder: Faris and Dunham revisited. Social Science and Medicine, 55, 1457-1470. 
Sjögren, E., Kristenson, M., \& Linquestgroup (2006). Can gender differences in psychosocial factors be explained by socioeconomic status? Scandinavian Journal of Public Health, 34, 59-68.

Sleath, B., West, S., Tudor, G., Perreira, K., King, V., \& Morrissey, J. (2005). Ethnicity and depression treatment preferences for pregnant women. Journal of Psychosomatic Obstetrics and Gynecology, 26, 135-140.

Smit, F., Bohlmeijer, E., \& Cuijpers, P. (2003). Wetenschappelijke onderbouwing depressiepreventie. Epidemiologie, aangrijpingspunten, huidige praktijk, nieuwe richtingen. [Scientific support for the prevention of depression. Epidemiology, targets, current practice, new directions.] Utrecht: Trimbos- instituut.

Snijders, T. A. B., \& Bosker, R. J. (1999). Multilevel Analysis: An introduction to basic and advanced multilevel modeling. London: Sage Publishers.

Sobocki, P., Jönsson, B., Angst, J., \& Rehnberg, C. (2006). Cost of depression in Europe. Journal of Mental Health Policy and Economics, 9, 87-98.

Solomon, A., Haaga, D. A., \& Arnow, B. A. (2001). Is clinical depression distinct from subthreshold depressive symptoms? A review of the continuity issue in depression research. Journal of Nervous and Mental Disease, 189, 498-506.

Southwick, S. M., Vythilingam, M., \& Charney, D. S. (2005). The psychobiology of depression and resilience to stress: Implications for prevention and treatment. Annual Review of Clinical Psychology, 1, 255-291.

Spinelli, M. G., \& Endicott, J. (2003). Controlled clinical trial of interpersonal psychotherapy versus parenting education program for depressed pregnant women. American Journal of Psychiatry, 160, 555-562.

Stathopoulou, G., Powers, M. B., Berry, A. C., Smits, J. A. J., \& Otto, M. W. (2006). Exercise interventions for mental health: A quantitative and qualitative review. Clinical Psychology: Science and Practice, 13, 179-193.

Steckler, A., \& Linnan, L. (2002). Process evaluation for public health interventions and research. San Francisco, CA: Jossey-Bass.

Steptoe, A., Kimbell, J., \& Basford, P. (1998). Exercise and the experience and appraisal of daily stressors: A naturalistic study. Journal of Behavioural Medicine, 21, 363-374.

Sterk, C. E., Theall, K. P., \& Elifson, K. W. (2006). Public health approach to depression and women: The case of the disadvantaged inner-city woman. In C. L. M. Keyes \& S. H. Goodman (Eds.), Women and Depression. A Handbook for the Social, Behavioral, and Biomedical Sciences (pp. 382413). New York: Cambridge University Press.

Ströhle, A. (2009). Physical activity, exercise, depression and anxiety disorders. Journal of Neural Transmission, 116, 777-784.

Stronks, K., Van de Mheen, H., Looman, C. W. N., \& Mackenbach, J. P. (1998). The importance of psychosocial stressors for socio- economic inequalities in perceived health. Social Science and Medicine, 46, 611-623.

Stuart, E. A., Perry, D. F., Le, H. N., \& Ialongo, N. S. (2008). Estimating intervention effects of prevention programs: Accounting for noncompliance. Prevention Science, 9, 288-298.

Tableman, B. (1987). Stress management training: An approach to the prevention of depression in low- income populations. In R. F. Munoz (Ed.), Depression Prevention: Research Directions (pp. 171-184). Washington: Hemisphere Publishing Corporation.

Tableman, B. (1989). Stress management training for low income women. Prevention in Human Services, 6, 259-284.

Taylor, S. E., \& Seeman, T. E. (1999). Psychosocial resources and the SES- health relationship. Annals of the New York Academy of Sciences, 896, 210-225.

Teelucksingh, C., \& Galabuzi, G. E. (2005). Impact of race and immigrants status on employment opportunities and outcomes in the Canadian labour market. Policy Matters (Metropolis), 22, 112.

Ten Have, M., Oldehinkel, A., Vollebergh, W., \& Ormel, J. (2003). Does educational background explain inequalities in care service use for mental health problems in the Dutch general population? Acta Psychiatrica Scandinavia, 107, 178-187. 
Ten Have, M., Vollebergh, W., Bijl, R. V., \& De Graaf, R. (2001). Predictors of incident care service utilisation for mental health problems in the Dutch general population. Social Psychiatry and Psychiatric Epidemiology, 36, 141-149.

Teychnne, M., Ball, K., \& Salmon, J. (2008). Physical activity and likelihood of depression in adults: A review. Preventive medicine, 46, 397-411.

Tezel, A., \& Gözüm, S. (2006). Comparison of effects of nursing care to problem solving training on levels of depressive symptoms in post- partum women. Patient Education and Counseling, 63, 64-73.

Theunissen, M., \& Nijsten, M. (2007). Buurtprofielen Maastricht 2006 [Neighborhood profiles Maastricht 2006]. Maastricht: Gemeente Maastricht.

Thoits, P. A. (1995). Stress, coping, and social support processes: Where are we? What next? Journal of Health and Social Behaviour, extra issue, 53-79.

Thoits, P. A. (2010). Stress and health: Major findings and policy implications. Journal of Health and Social Behavior, 51, S41.

Thomas, J. L., \& Brantley, P. J. (2004). Factor structure of the Center for Epidemiological Studies Depression scale in low- income women attending primary care clinics. European Journal of Psychological Assessment, 20, 106-115.

Thomas, J. L., Jones, G. N., Scarinci, I. C., Mehan, D. J., \& Brantley, P. J. (2001). The utility of the CES-D as a depression screening measure among low- income women attending primary care clinics. International Journal of Psychiatry in Medicine, 31, 25-40.

Timmerman, I. G. H., Emanuels-Zuurveen, E. S., \& Emmelkamp, P. M. G. (2000). The Social Support Inventory (SSI): A brief scale to assess perceived adequacy of social support. Clinical Psychology and Psychotherapy, 7, 401-410.

Tsang, H. W., Chan, E. P., \& Cheung, W. M. (2008). Mind-body therapy. Effects of mindful and non-mindful exercises on people with depression: A systematic review. British Journal of Clinical Psychology, 47, 303-322.

Turner, R. J., \& Lloyd, D. A. (1999). The stress process and the social distribution of depression. Journal of Health and Social Behavior, 40, 374-404.

Twisk, J. W. R. (2006). Applied multilevel analysis: a practical guide. Cambridge: University Press.

Üstün, T.B., Ayuso-Mateos, J.L., Chatterji, S., Mathers, C., \& Murray, C.J.L. (2004). Global burden of depressive disorders in the year 2000. British Journal of Psychiatry, 184, 386-392.

Van de Velde, S., Bracke, P., \& Levecque, K. (2010). Gender differences in depression in 23 European countries. Cross-national variation in the gender gap in depression. Social Science $\mathcal{E}$ Medicine, 71, 305-313.

Van den Berg, M., \& Schoemaker, C. G. (2010). Effecten van preventie. [Effects of prevention.] Houten: RIVM

Van der Waerden, J. E. B., Hoefnagels, C., \& Hosman, C. M. H. (2011). Psychosocial preventive interventions to reduce depressive symptoms in low-SES women at risk: A meta-analysis. Journal of Affective Disorders, 128,10-23.

Van der Waerden, J. E. B., Hoefnagels, C., Jansen, M. W. J., \& Hosman, C. M. H. (2010). Exploring recruitment, willingness to participate, and retention of low-SES women in stress and depression prevention. BMC Public Health, 10, 588-596.

Van der Waerden, J. E. B., Jansen, M. W. J., Hoefnagels, C., \& Hosman, C. M. H. (in press). Rationale and process evaluation of the Exercise without Worries intervention: do disadvantaged women appreciate a synergetic exercise and psycho-educative program? International Journal of Person Centered Medicine

Van Sonderen, E. (1993). Het meten van sociale steun met de Sociale Steun Lijst-Interacties en Sociale Steun Lijst Discrepanties: een handleiding [Measuring social support with the Social Support List-Interactions and Social Support List-Discrepancies: a manual]. Groningen: Noordelijk Centrum voor Gezondheidsvraagstukken.

Voordouw, I., \& Kramer, J. (2001). Implementatie van de cursus Omgaan met depressie in de preventieve geestelijke gezondheidszorg. Resultaten van de tussentijdse evaluatie [Implementation of the Coping With Depression course in the preventive mental health care. Results of a interim evaluation]. Utrecht: Trimbos-instituut. 
Ware, N. C., Tugenberg, T., Dickey, B., \& McHorney, C. A. (1999). An ethnographic study of the meaning of continuity of care in mental health services. Psychiatric Services, 50, 395-400.

Weinreb, L., Goldberg, R., \& Perloff, J. (1998). Health characteristics and medical service use patterns of sheltered homeless and low-income housed mothers. Journal of General Internal Medicine, 13, 389-397.

Weinreb, L., Perloff, J., Goldberg, R., Lessard, D., \& Hosmer, D. W. (2006). Factors associated with health service utilization patterns in low-income women. Journal of Health Care for the Poor and Underserved, 17, 180-199.

West, S., King, V., Carey, T. S., Lohr, K. N., McKoy, N., Sutton, S. F., \& Lux, L. (2002). Systems to Rate the Strength of Scientific Evidence. Evidence Report/ Technology Assessment No 47 (Prepared by the Research Trial Institute - University of North Carolina Evidence-based Practice Center under Contrast No 290-97-0011) Rockville, MD: Agency for Healthcare Research and Quality.

Wheaton, B. (2010). The Stress Process as a successful paradigm. In W. R. Avison \& C. S. Aneshensel \& S. Schieman \& B. Wheaton (Eds.), Advances in the conceptualization of the Stress Process: Essays in honor of Leonard I. Pearlin. New York Springer.

Whisman, M. A. (1990). The efficacy of booster maintenance sessions in behavior therapy: Review and methodological critique. Clinical Psychology Review, 10, 155-170.

White, K., Kendrick, T., \& Yardley, L. (2009). Change in self-esteem, self-efficacy and the mood dimensions of depression as potential mediators of the physical activity and depression relationship: Exploring the temporal relation of change. Mental Health and Physical Activity, 2, 44-52.

WHO. (2000). Women's mental health: an evidence based review. Geneva: World Health Organization.

WHO. (2001). World Health Report 2001: Mental health: New understanding, new hope. Geneva: World Health Organization.

WHO. (2010). Global recommendations on physical activity for health. Geneva: World Health Organization.

Wiggins, M., Oakley, A., Roberts, I., Turner, H., Rajan, L., Austerberry, H., Muijca, R., Mugford, M., \& Barker, M. (2005). Postnatal support for mothers living in disadvantaged inner city areas: A randomised controlled trial. Journal of Epidemiology and Community Health, 59, 288-295.

Wiggins, M., Oakley, A., Roberts, I., Turner, H., Rajan, L., Austerberry, H., Mujica, R., \& Mugford, M. (2004). The social support and family health study: A randomised controlled trial and economic evaluation of two alternative forms of postnatal support for mothers living in disadvantaged inner-city areas. Health Technology Assessment, 8, 1-134.

Willemse, G. R. W. M., Smit, F., Cuijpers, P., \& Tiemens, B. G. (2004). Minimal-contact psychotherapy for sub-threshold depression in primary care: Randomised trial. British Journal of Psychiatry, 185.

Willige, G. v. d., Schreurs, P., Tellingen, B., \& Zwart, F. (1985). Het meten van "life events": Recent meegemaakte gebeurtenissen (VRMG) [Measuring life events: recently experienced events]. Nederlands Tijdschrift voor Psychology, 40, 1-19.

Wilson, D. B., \& Lipsey, M. W. (2001). The role of method in treatment effectiveness research: Evidence from meta- analysis. Psychological Methods, 6, 413-429.

Zayas, L. H., McKee, M. D., \& Jankowski, K. R. B. (2004). Adapting psychosocial intervention research to urban primary care environments: A case example. Annals of Family Medicine, 2, 504-508.

Zich, J. M., Attkisson, C. C., \& Greenfield, T. K. (1990). Screening for depression in primary care clinics: Tthe CES-D and the BDI. International Journal of Psychiatry in Medicine, 20, 259-277.

Zlotnick, C., Miller, I. W., Pearlstein, T., Howard, M., \& Sweeney, P. (2006). A preventive intervention for pregnant women on public assistance at risk for postpartum depression. American Journal of Psychiatry, 163, 1443-1445.

Zlotnick, C., Johnson, S.L., Miller, I.W., Pearlstein, T., \& Howard, M. (2001). Postpartum depression in women receiving public assistance: Pilot study of an interpersonal-therapy-orientated group intervention. American Journal of Psychiatry, 158, 638-640. 
Dankwoord 


\section{Dankwoord}

Hoewel de naam Bewegen zonder Zorgen anders doet vermoeden, heeft dit onderzoeksproject te maken gehad met kleine en grotere zorgen. Graag wil ik dan ook iedereen bedankten die heeft bijgedragen aan het tot stand komen van dit proefschrift.

Clemens Hosman, Cees Hoefnagels en Maria Jansen, jullie zochten destijds het spreekwoordelijke "schaap met vijf poten". Bedankt voor het vertrouwen dat jullie mij schonken bij het uitvoeren van dit onderzoek en jullie bijdrage hieraan in de afgelopen jaren. Truus Gelissen, als lid van de projectgroep was ook jij belangrijk voor het welslagen van dit project.

Bewegen zonder Zorgen was in goede handen bij de cursusleidsters. Wilmie Lanen, Soosan Boothe, Nicole Ebben, Claudia van Dam, Eelke Sistermans en Henriette Vermeer, jullie enthousiasme en kundigheid waren onmisbaar voor de uitvoering van de cursus.

Dank ook aan alle deelneemsters die meerdere keren de tijd hebben genomen voor het invullen van de vragenlijsten. Aan de dataverzameling hebben verschillende assistent onderzoekers een bijdrage geleverd: Stephanie van Bragt, Selma van Diest, Jantha de Gelder, Madelon van Hees, Nelleke Heinemans, Wieteke Janssen, Joyce van Leendert, Nadine Mouchart, Danou Nijsten, Mariette Prevoo-Notten, Lilianne Tellier-Notten en Lili van Wielink. Zonder jullie hulp zou het praktisch onmogelijk zijn geweest om alle screenings en vragenlijsten af te nemen. In het bijzonder wil ik Jet Dingemans noemen die me met haar rol als student assistent veel regelwerk uit handen heeft genomen.

Collega's en oud collega's van Gezondheidsbevordering, Klinische Psychologie en Context, bedankt voor alle gezellige momenten tijdens en ook buiten het werk.

Evelien en Roos, wat fijn dat jullie vandaag naast mij staan als paranimfen. Bedankt voor het delen van lief en het verzachten van leed.

Familie en vrienden, bedankt voor jullie betrokkenheid en interesse in de voortgang van mijn onderzoek.

Aurélien, cette thèse était la raison d'aller travailler le matin, tu es ma meilleure raison pour rentrer le soir....Merci pour tout. 
Curriculum Vitae 
220 


\section{Curriculum Vitae}

Judith van der Waerden was born on January 18, 1979 in Dordrecht, the Netherlands. After completing secondary school in 1997 (Johan de Witt Gymnasium, Dordrecht), she began her studies in Health Education and Promotion at the Faculty of Health Sciences of Maastricht University, the Netherlands. In 2001 she graduated and in the following three years she obtained an additional degree in the discipline of Marketing Communications at Radboud University Nijmegen, the Netherlands. From 2004 to 2011 Judith worked as a PhD candidate at the department of Health Promotion at Maastricht University. The results of this research are presented in this thesis. Besides her research work, she was responsible for various educational activities related to the prevention of mental disorders and mental health promotion. From April 2009 to November 2010 she worked part-time as a university teacher at the Clinical Psychology department of Radboud University Nijmegen. As of February 2011, Judith works as a researcher for the Knowledge center for Mental disorder prevention of Context, the prevention department of the Parnassia Bavo Group in The Hague. 
